\title{
ENCYCLOPÉDIE SCIENTIFIOUEL
}

PUBLIEE SGUS LA OIREETION DU DA TOULOUSE.

BIBLOTHEิQUี

DES INDUSTRIES PHYSIQUES
DIRECTEUR

H. CHAUMÁt

\section{Instruments optigues}

d'Observation et de Mesure

\author{
PAR \\ TULES RAIBAUD
}

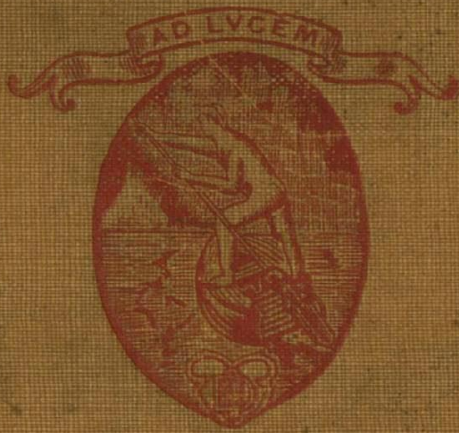

Octave DOIN \& Fris. Eniteurs. Paris IRIS - LILLIAD - Université Lille 1 
IRIS - LILLIAD - Université Lille 1 
Octave DOIN et Fils, éditeurs, 8, place de l'0déon, Paris.

\title{
ENCYCLOPÉDIE SCIENTIFIQUE
}

Publlée sous la direction du Dr Toulouse

\author{
BIBLIOTIIEQUE \\ DES INDUSTRIES PHYSIQUES \\ Directeur : H. Chaumat \\ Sous-directeur de I'École supérieure d'Électricité.
}

Il n'est réellement pas besoin de montrer à quel point est important le domaine des applications de Ia Physique dont relève presque toute l'industrie moderne.

Mais, dans cet immense domaine, il est une part considérable qui se tronve traitée dans la Bibliothèque de Mécanique appliquée, en particulier toutes les applications particulières de la Thermodynamique. La prédominance des données mécaniques dans la construction des moteurs et des machines innombrables servant au travail des métaux et des bois, aux divers modes de traction, etc., a nécessité cette division générale dans le domaine des applications de la Physique.

Aussi, à part un petit nombre de volumes, cette Bibliothèque cst-clle essentiellement consacrée à la branche de 
la Physique qui tend bien à devenir la plus importante dans l'industrie, à l'Électricité.

Dans cette branche, les progrès sont actuellement des plus rapides; la captation de l'énergie sous la forme électrique, particulièrement maniable, et sa distribution amèneront peut-être dans un avenir prochain des transformations économiques profondes, telles que le développement des ateliers familiaux. C'est aussi gràce aux facilités de transport de l'énergie électrique qu'ont pu être utilisées des forces naturelles pratiquement inépuisables, comme les chutes d'eau des régions montagneuses, qui alimentent en énergie des villes, souvent éloignées à des centaincs de kilomètres, qui leur donnent la lumière et leur assurent l'activité. Et les circulations électriques, qui servent à la traction d'innombrables véhicules, permettant aux hommes de se déplacer avec une extrême rapidité, et qui assurent aussi entre des points quelconques de la terre un échange immédiat de pensées, se doublent maintenant du libre transport des ondes électriques qui, sans être emprisonnées dans un réseau, rendent particulièrement simples les communications télégraphiques; la téléphonie sang̣ fil débute même déjà, et l'on conçoit la possibilité, - que des essais ont déjà démontrée, - d'alimenter d'énergie à distance des éléments mobiles, des torpilles qu'on dirigerait, des navires qu'on éclairerait.

Le róle capital, et sans cesse croissant, de l'électricité dans la vie industrielle contemporaine exigeait donc une place particulièrement importante dans cette Encyclopédie pour les volumes consacrés à ses applications. Et l'on risque plus à cet égard de pécher par défaut que par excès. Mais il faut bien s'attendre à ce que les cadres de cette Bibliothèque s'élargissent ultérieurement au fur et à mesure des progrès nouveaux. Telles applications, que l'on ne soupconne mème pas aujourd hui, surgiront sans doute demain, et l'on devra dès lors leur faire une place. 
C'est là ce qui fait justement l'utilité de cette Encyclopédic, qui cherche à rester vivante, en s'adaptant à la vie méme de la science.

Les volumes seront publiés dans le format in-18 jésus cartonne; ils formeront chacun 350 pages environ avec ou sans figures dans le texte. Le prix marques de chacun d'eux, quel que soit le nombre de pages, est fixé \& 5 francs. Chaque volume se vendra séparément.

Voir, á la fin du volume, la notice sur l'ENGXCLOPÉDIE SCIENTIFIQUE, pour les conditions générales de publication. 
IRIS - LILLIAD - Université Lille 1 


\section{TABLE DES VOLCMES \\ ET LISTE DES COLLABORATEURS}

Les volumes publiés sont indiqués par un ".

1. L'Électricité industrielle. Thèories générales. 2 vol.

2. Le Ferro-Magnétisme; applications, par Jouaust, Ingénicur au Laboratoire central d'Électricilé.

3. Les Matériaux au point de vue électrique.

4. Los Constructions électriques à courant continu. 2 vol.

5. Les Constructions électriques à courant alternatif. 2 vol.

6. Les Rhéostats.

7. Transport ot distribution de l'énergie electrique. Principes généraux.

8. Les Canalisations électriques.

9. Les Tableaux de distribution et l'Appareillage électrique.

10. Les Moteurs électriques.

11. La Commande électrique des ateliers.

12. L'Électricité dans les mines.

13. La Traction électrique.

14. L'Éclairage électrique, par Camille LB́onand, Ingénicur de la Compagnie Thomson-Houston.

15. La Télógraphio et la Téléphonio.

16. La Télógraphio sans fils et les applications des oscillations électriques, par J. Tissó, professeur à l'École navale.

17. Les Applications de l'électricité à la Guerre et à la Marine, par Henra Royer, Ingénieur de la Marine, professeur da l'École supérieure de la Marine.

18. Les Applications usuelles de l'électricité. 
39. L'Électro-Chimie.

20. Le Four électrique.

21. Les Accumulateurs électriques.

22. Los Essais de récoption.

23. I'Usine hydro-électrique.

24. La Thermodynamique appliquee.

25. La Liquéfaction des gaz.

26. La production industrielle du froid.

27. La production industrielle des hautes températures.

28. L'Éclairage.

29. Le Chauffage.

30. Instruments d'acoustique.

-31. Instruments optiques d'observation ot do mesures, par le Capitaine Rarbaud, Chef du Laboratoire de Physique d la section technique de l'Artilerie. 


\title{
ENCYCLOPÉDIE SCIENTIFIQUE
}

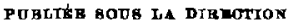

du Dr TOULOUSE, Directeur de Lahoratoire l'b́cnle des Hautes-Études.

Secrétaire général : H. PIÉRON, Agrégé de l'Université.

BIBLIOTHEQUE DES INDUSTRIES PHYSIQUES

Directeur : H. Chaumat

Sous - directeur de l'École superieure d'Électricité.

\section{INSTRUMENTS OPTIQUES}

IRIS - LILLIAD - Université Lille 1 
IRIS - LILLIAD - Université Lille 1 


\section{INSTRUMENTS 0PTIQUES}

D'OBSERVATION ET DE MESURE

PAR

JULES RA I B A U D

UAPITAINB D'ARTILLKRIK

Avec 144 figures dans lo texte

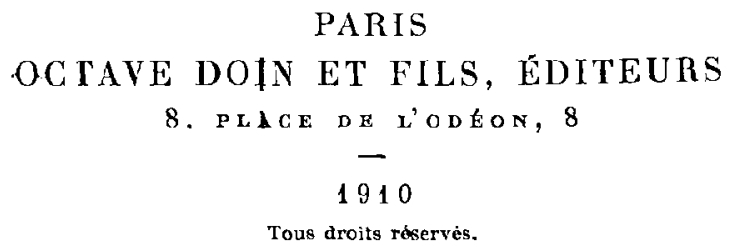

IRIS - LILLIAD - Université Lille 1 
IRIS - LILLIAD - Université Lille 1 


\title{
INTRODUCTION
}

\author{
GENERALITFS
}

x. Caractère des instruments d'observation. Substituer à la contemplation directe des objets l'examen d'images optiques convenables, soit réclles, soit virtuelles, tel est lo róle des instruments optiques d'observation. Ces apparcils mettent essentiellement en ouvre les propriétés rélléchissantes des surfaces polics, et les propriétés réfringentes des corps transparents.

Lcur théoric est régie, à peu près exclusivernent, par une loi simple, à laquelle Sncllius et Descartes ont attaché leurs noms : Ic changement de direction d'un rayon lumineux rencontrant la surface de séparation de deux nilieux réfringents s'effectue dans le plan du rayon incident et de la normale, issue du point d'incidence, à la surface séparatrice; la direction du rayon rófracté est telle que le rapport du sinus de l'angle d'incidence $i$ à celui de l'angle de rófraction $r$ est une constante $n$, - indice de réfraction du second nilieu par rapport au premier, - ne dépendant que de la nature des milieux, et non de la grandeur de l'angle d'incidence.

Les recherches subséquentes ont appris que cet indice relatif $n$ était égal au rapport des indices relatifs $n_{2}$ et $n_{1}$ du

Instruments optiques. 
second et du premier milieu par rapport à un troisième quelconque; et, par suite, égal au rapport de leurs indices absolus (ce qualificatif s'appliquant lorsque lo troisieme milieu, dont le choix reste toujours libre, est le vide). Lindice $n$ est aussi égal au rapport des vitesses de propagation des ondes lumineuses dans le milieu d'incidence et celui de réfraction, ou au rapport des Iongucurs d'ondes, dans los mèmes substances, de la radiation lumineuse en jeu.

$$
\frac{\sin i}{\sin r}=n=\frac{n_{z_{-}}}{n_{1}}==\frac{v_{1}}{v_{2}}=\frac{\lambda_{1}}{\lambda_{2}} \text {. }
$$

\section{Classification des instruments d'observation.}

- Toute classification, étant plus on moins artificiclle, reste incomplète el se trotive généralement peu satisfaisante; sa nóccssité s'impose cependant dans un exposć didactirue. Sous le bénéfice de cette double remarque, nous répartirons dans les trois catégrories ci-après les instruments dont nous nous occuperons.

En premier lieu, les instruments destinés à l'observation des objets ćloignés; ils sont représentés par les longues-vues, lunettes d'approche terrestres, lunettes astronomiques, télescopes catoptriques. Ils pourraient itre rangés sous le nom générique de télescopes (ainsi qu'on le faisait jadis), si ccluici n'était pas actuellcment à peu près réservé aux instruments astronomiques it miroirs courbes. Lne subdivision spéciale renfermera, sous le nom de jumelles, les appareils utilisant la vision binoculairc.

Le seconde catégoric comprendra Ies microscopes, en ćtendant cette désignation aux loupes simples ou composées (dites parfois microscopes simples). Les instruments de cette nature sont utilisés pour l'examen des objets rapprochés.

Les différentes variétés de l'objectif photographique constitueront le troisième groupe. Cet organe, le photo-objectif, d'applications si varićes, peut, logiquement, etre considéré 
comme un véritable instrument d'observation. Tandis que les appareils des deux premières catégories soumettent, en définitive, à l'examen visuel une image opticpue virtuelle, l'objectif photograplique donne une image réelle, qui peut être observée directement ou non; malgré cette différence, on pourrait, suivant les circonstances, faire rentrer le photoobjectif dans l'un on l'autre des deux groupes précédents, si son róle habituel n'était pas de permettre la réalisation, la matérialisation de l'image produite. Grâce à la plaque sensible, l'objectif devient un merveilleux instrument d'observation, ofl'aut sur les aulres, _ à côté, il est rrai, de certains inconvénients, - l'avantage indiscutable de conserver une trace fidèle de l'objet examíné. D'ailleurs l'emploi de la plaque photographique exige, pour le photo-objectif, des proprićtés spéciales qui justifient sa séparation d'avec les instruments énumérés plus haut.

3. Constitution généralo des instruments d'observation. - Les éléments optiques utilisés sont des surfaces réfléchissantes et des masses réfringentes.

Ces dernières sont constituées par des corps transparents, perméables à la lumière, dont les deux surfaces utiles dites encore efficaces ou actives, sont polies et de formes régulières. Lorsque ces surfaces, - ou au moins l'une d'elles, Iautre pouvant être plane, - sont sphériques, l'élément réfringent porte le nom de lentille, et comporte six variétés principales bien connues; si elles sont planes et inclinćes l'urıe sur l'autre, on sait que l'élément est désigné sous le nom de prisme; enfin, deux taces planes et parallèles limitent une glace ou lame.

On a utilisé encore, quoique exceptionnellement, des lentilles dont les faces étaient des splières légèrement et systématiquement déformées ; chaque surface reste de révolution autour de l'axe de l'élément, et sa méridienne est une parabole, de degré plus ou moins élevé. osculatrice au cercle générateur de la sphère, en son sommet. Quant aux 
lentilles à surfaces elliplique, hyberbolicue, etc., on ne peut considérer que comme des curiosités sans grand intérêt les rares instruments dans lesquels elles ont été employées.

Les surfaces non actives des éléments réfringents, généralement surfaces d'appui brutes, ou mieux dépolies, sont des cylindres concentriques à l'axe, dans les lentilles : dans les prismes et dans les glaces, nlles sont symétrifues par rapport aux faces d'entrée et de sortic, à moins que la constitution spéciale de l'apparcil no leur impose une autre orientation.

Les matières - homogènes, et, autaut que possible, isotropes - utilisées dans ces éléments réfringents sont, dans la majorité des cas, les produits artificiels si variés désignés sous le nom de verres d'optique : crown, flint, glace, cristal, etc. Certaines substances naturelles, biréfringentes, - cristal de roche, quartz, spath d'lslande, - ont été mises à contribution, mais plus spécialement dans les apparcils de mesure. Quant aux liquides, adjurants précicux dans certaines circonstances (observations au microscope, par exemple), ils p'ont pas, jusqu'ici, répondu aux espoirs qu'on avait fondés sur cux.

Signalons encore, comme matières réfringentes, les rísines, - baume de Canada, térébenthène, - employées en quantités prescue infinitésimales dans Ia liaison des substances rélringentes solides.

Les surfaces réfléchissantes des instruments optiques sont plus spécialement métalliques : clles sont constituées soit par la surface polie d'une masse en alliage spécial, soit par une mince couche d'argent brunie et polie, déposée sur une forme convenable en verre. Ces surfaces portent le nom bien connu de miroirs, avec les qualificatifs particuliers de plan, convexe, concave, sphérique, parabolique, etc.

On emploie aussi comme surfaces réfléchissantes, surtout 
quand il s'agit de plans de laibles dimensions, la surface de séparation d'une masse réfringente et de l'air, soit en utilisant le phénomène de la réflexion totale, soit en se contentant parfois de celui de la réflexion partielle. Les surfaces de réflexion font parlie de prismes en verre, ou même de polyèdres comprenant au moins, en dchors de la surface réfléchissante, deuy faces polies, une d'entréc, une de sortic, que les rayous attaquent le plus normalement possible.

4. - Le seul caractère à peu près général que présentent les instruments optiques, relatiroment à la disposition de leurs éléments réfléchissants et réfringents, cst clue l'ensemble de ces dernicrs constitue, dans chaque appareil, un système centré. Les centres des surfaces actives des lentilles, ceux des miroirs, sont situćs sur une mêne dioite. dite axe du système ou de l'instrument. Sur ce dernier axe sont appliqués aussi ceux des surfaces de révolution non spliéricques.

L'axe du systime n'est généralement brisé que par les rétlexions qui se produisent sur les éléments plans ; l'instrument comprend alor's plusieurs systènes centrés sur des axes différents. Yais, en laisant l'abstraction légitime des réflexions planes, le système composé rederịent unique.

Quant aux deviations et aux dédoublements daxe produits par des lames ou des prismes réfringents, ils constitucnt des cas d'espice. Il est nécessaire, par l'examen de linstrument, de voir s ill est possible de considérer ce dernier comme un systime centré unique (après suppression fictive des éléments déviateurs), ou s'il est indispensable de l'envisager comme un composé de sıstèmes centrés.

Les divers éléments optiques d'un instrument sont en général portés par une monture, plus on moins rigide, qui forme le corps de l'appareil. C'est à cette monture que sont fixés les seuls organes mécomiques communs ì tous les ins- 
truments : les diaphragmes, lames opaques, minces, percées d'une ouverture généralement circulaire, centrée sur l'axe et dans un plan perpendiculaire à celui-ci. Ils comportent trois variétés : les diaphragmes de limitation, qui règlent l'amplitude des faisceaux traversant l'instrument, formant écran pour les rayons jugés nuisibles; les diaphragmes de champ qui arrètent les faisceaux provenant de points objets dont la vision à travers l'instrument serait imparfaite; les diaphragmes de clarté, s'opposant à la propagation de faisceaux qui, issus d'objets non soumis à l'examen, altéreraient les qualités de l'image observée.

l.es montures des verres penvent remplir le róle de diaphragme de limitation.

En principe, il n'y a qu'un seul diaphragme de limitation et un seul de champ efficaces. Quand il en existe plusieurs remplissant le même rỏle, ils doivent se correspondre : chacun d'eux agissant, réellement on virtuellement, sur les partics homologues du faisceau.

5. Instruments de mesure. - On transforme un appareil opticue d'observation en instrument de mesure en lui adaptant les organes mécaniques on optiques qui permettent d'utiliser les propriétés métriques des images on les conséquences optiques de la déformation géométrique de l'appareil primitif.

Ponr satisfaire aux conditions recuuses, la modification en question exige parfois la combinaison en un seul appareil d'instruments de mème espèce ou de variétés différentes; dans d'autrcs cas, elle réduit l'instrument au rôle d'un appareil plus complexe. Mais, dans la sphère des transformations pratiques, un fait paraît dominer : la modification en instrument de mesure ne s'opère, dans les meilleurs conditions possibles, qu'an détriment de quelques-unes des qualités de l'appareil, en tant qu'appareil d'observation.

5. Classification des instruments de mesure. -Le rayon lumineux, - on. mieux, le faiscenu infiniment 
délié, le pinceaa, - constitue la réalisation la plus parfaite de la notion géométrique de direction ; deux directions déter minent un angle; si l'on joint à la mesure des angles la connaissance d'une longueur, - d'une base, - on aura tous les éléments pour évaluer les distances.

Ces remarques nous serviront de guide pour ćtablir une classification des instruments de mesure; répartition qui sera quelque peu factice, mais qui semble acceptable, à condition de ne pas s'astreindre à des divisions trop tranchées.

Sous cette réserve, nous distinguerons :

Les instruments, - généralement simples organes d'appareils complexes, - dont le seul but est de réaliser optiquement une ligne droite; ils seront rangés sous la rubrique de viseurs;

Ceux dont le ròle essentiel consiste dans la mesure des écarls angulaires, et qu'on peut dénommer goniomètres.

Parmi les appareils spécialement organisés pour la mesure des distances, nous classcrons séparément : d'abord, ceux pour lesquels la base est en dehors de l'instrument, et généralement voisine de l'objet; - ces instruments, qu'on pourrait appeler stadinétriques, se distinguent souvont à peine do ceux signalés dans la catégorie précédente;

Ensuite, les appareils qui contiennent la base linéaire; co sont les instruments à parallaxe, que nous engloberons sous la dénomination de télémètres.

Dans une catégorie spéciale, tant en raison de leur emploi que de leur organisation optique, nous rangerons les instruments qui utilisent le principe si fécond de l'auto: collimation.

7. Instruments de mesure utilisant les phénomènes de l'optique physique. - Ia classification qui précède est loin de comprendre toute la sćrie des instruments de mesure; olle ne renferme que ceux qui relivent le plus directement de l'optique géométrique, de la loi de 
Descartes. D’autres apjpareils mettent ì contribution les phénomènes si variés et si délicats de loptirpue physique. Le défaut d'espace nous oblige à les passer sous silence.

\section{IISTORIQLE SOMMAIRE}

8. - On peut, à la rigueur, f́are remonter jusquaux dernières années du xur siècle, époque de l'invention des besicles, l'origine des instruments d'opticjue. Mais il faudra Jaisser s'écouler encore trois cents ans avant de rencontrer. hors la loupe, un appareil optique digne de ce nom.

Les découvertes se succédent alors rapidement. Le xvur siècle voit surgir, dans une forme primitive, il est vini, toute la variété des télescopes et des microscopes; le xvm" siècle les perfectionnera, dés surtout que la découverte de l'achromatisme aura ouvert une voie nouvelle. Une autre poussée se produira dans le courant du siècle suivant, amenée par l'útude des problèmes que soulive lá photographie, ot aidée par le díveloppenent intense des arts mécaniques.

9. L'optique chez les Anciens, et avant le XIVe, siècle. - Excellenls géomètres, remarquables observateurs, Ies anciens Gress ne furent, aulant qu'on en pent juger par ce qui nous reste d'cux, que de médliocres ou dédaigneux expérimentateurs. Ils ne paraissent. avoir connu aucur instrument optique (70); sinon ils l'auraient utilisé dans leurs observations astronomiques, et transmis ensuite aux Arabes. Quant a leurs connaiswances. relativement aux phénomènes lumineux, elles sont toujours rudimentaines ou vagues, sourent inoxactes. L'opticue d'un certain Euclide, - pas le géonètre, - n’infirmera pas cette opinion; cc ne pourrait être que le traité attribué à A rchimède, sil n'était entièrement perdu, - à condition fentefois qu'il eùt été composé par ce grand homme. 
Les connaissances opliques des anciens proviennent de l'observation, quand elles ne consistent pas en idées géométriques préconçues. La véritable cause des éclipses (conséquence de la propagation rectiligne de la lumière), l'origrine de la clarié de la Lune sont connues de Pyllagrore (- 580 ). Paton et son école (-3jo curiron) sont en possession de la loi d'égralité des angles dincidence et de réflexion, vérité proliablement découverte par la seule géométrie (loi du trajet minimum, que reprendront plus tard Descartes et Fermat). Lidóe do la réfraction, - réfraction astronomicue, apparait dans losidionios, se précise clecz son disciple Cléomède, qui la rapproche de la réfraction dans l'eau, pour explicpuer la différence des grandiurs apparentes d'un astre à différentes élévations sur l'horizon. Plutar(jue, Ptolémée (fin du $\mathrm{I}^{\text {or }}$ siècle) signalent nettement le pliénomène de la réfraction de la lumière passant de l'air dans l'eau.

- Les Romains, d'esprit bien moins scientifique gue les Gíecs, ne scmblent aroir possédé aucune notion d'oplique : un curieux des choses naturelles, Sénèque ${ }^{1}$, signale cependant l'effet grossissant d'une boule de verre remplie d'eau, et les coulcurs de l'iris produites par uno baguette de verre prismatique; un. compilateur, Pline ${ }^{2}$, rapporte que Yéron regardait a travers une émeraude concave les combats de gladiateurs. Il est toutefois difficile de trouver dans ce: remarpues la trace de la loupe, ou des besicles.

l'endant la plus grande partie du moyen ige, loptique n'effectue aucun progrès. Les savants arabes Al-Hizen, AlfaRabius, Ibn-Ileitem composent leurs lives des vestiges de l'antiquité. Vitellion copie Al-IIazen, et ébanche la théorie de l'arc-en-ciel et des parhélies. La plupart essayent, inlructueusement, d'ćlablir la loi de la réfraction.

ı. L'invention des besicles. - Jusqüici tout

1 Suxigre, Questions naturelles, lirre I.

2 Prise, lirre XxXVII. 
phénomène optique provient de l'observation. Le fait parait tenir à ce que, si le verre était connu depuis une haute antiquité, son travail était rudimentaire; le coulage et le soufflage de la matière vitreuse suffisaient à toutes les exigenees. Le désir d'obtenir des glaces parfaitement polies incita probablement les Vénitiens à employer pour le polissage du verre la méthode d'usure lente utilisće dans le travail des pierres précieuses. L'opération du polissage réalisée, l'obtention de surfaces sphériques n'offrait aucune difficulté sérieuse.

La première application des lentilles polies s'adressa à l'amélioration des vues fatiguées. L'histoire ne peut, avec sùreté, associer le nom d'un inventeur à celui des besicles ou lunettes; elle hésite entre le seigneur Salvino Armato degli Armati (de Florence), le Frère Alexandre de Spina (des Frères Prêcheurs de Pise); mais l'époque de l'apparition des lunettes peut, sans crainte d'erreur, ètre fixće aur dernier quart du xin ${ }^{\mathrm{B}}$ siècle (64).

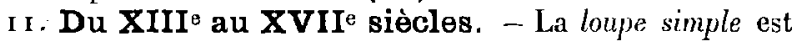
contemporaine des besicles; peut-ctre aussi la loupe composée de deux verres à faible intervalle. D'ailleurs Al-Mazen avait fait, au sujet d'un segment de verre coulé, une remarque analogue à celle formulée par Sénèque sur la boule pleine d'eau; il avait mème ajonté que le verre était d'autant plus grossissant qu'il approchait davantage de la sphère entière.

La loupe reste à peu près le seul instrument optique jusqu'au commencement du xvi" siècle. Dans l'intervalle, quelrfues notions se précisent. Maurolyc assimile le cristallin à une lentille, mais n'arrive pas jusqu'à la conception des images rétinicnnes. Porta découvre la chambre noire en disposant une lentille convexe dans l'ouverture de la chambre obscure; mais, quoique médecin et anatomiste, il ne se rósout pas à assimiler l'oil à sa chambre noire.

I 2. L'invention du télescope et du microscope. 
- Un heurcux hasard, - un amusement d'enfants, suivant une légende qui est peut-être de l'histoire, - fait découvrix, au commencement du xvrro siècle, la combinaison qui constitue la première lunetle d'approche : objectif convexe, oculaire concave. L'origine de l'instrument est hollandaise; sa paternité est moins ccrtaine : clle se répartit sur Zacharie Jans, Jean Lapprey, lunettiers à Middelbourg; et Jaccues Metius. Sur la sinple annonce de sa découverte, Galilée la réalise (16og) à Venise, construit une lunette grossissant trente-trois fois et commence à publicr son Sidereus Nuntius.

La mème époque voit naître le mirroscope; les progrès du verre, permettant d'obtenir des lentilles de court foyer, en rendent la constuction possible. On l'attribue, avec des motifs également plausibles, tantôt à Zacharie Jans, tantòt ì Corneille Drebbel.

La lunette astronomique suit, à pou d'intcrvalle, les instruments précédents; on peut, à elle, donner avec certitude, un père illustre : Kepler; elle présente, de plus, ce caractère historique important qu'elle constitue le premier système optique théoriquement conçu avant d'être réalisé. Kepler, dans son Traité d'Astronomie, aborde l'Optique; il étudie l'mil, qu'il assimile très justement à la chambre noiro de Porta, reconnaît les rôles de la cornée, du cristallin et des liumeurs, constate le renversement des imarges rétiniennes. Il explique les proprićtés des besicles, décrit le télescope à deux verres convexes et en expose les effets.

Kepler laissa à un de ses disciples, le Père Schoiner, le soin de réaliser l'instrument conçu; peut-ìtre mème lui indiqua-t-il aussi le télescope redresseur à trois verres, la première lunette terrestre à verres convexes, qui fut également exćcuté. Les inconvénients de ce dernier appareil (sa grande longueur, la déformation des images, leurs irisations) fixrent atténués par le Père de Rheita, qui imagrina de remplacer la lentille centrale par le système véhiculaire de deux lentilles. 
Au mêne savant on attribue l'idée d'avoir associé deux lunettes pour la vision binoculaire. Mais un fabricant frargais l'arail précédé dans cetle voic (44).

I3. La loi de Snellius-Descartes. - La loi exacts de la réfraction avait échappé ì Kepler, comme à Al-llazen. Le célcbbre astronome lui avait substilué la règle de proportionnalité entre. les angres d'incidence el de réfiaction : elle suf-' fisait pour l'explication qualitative et, dans une cerlaine mesure, quantilative des phénomines. L ne loi rigourcuse était pourtant indispensable pour pouvoir transformer, en lui appliquant la méthode géométrique, l'art de l'oplicien science. Kepler aurait pu connailre la loi, car elle était enseignée publiquement a Leyde, par un de ses contemporains, Milebrod Snellius. Descartes en nodilia l'énoncé, montua qu'elle déconlait du principe du chemin mininum, la publia et l'utilisa dans la recherche des surfaces aplanétiques. Ia postérilé a rendu juslice an grand philosophic en altribuant son nom à la loi des sirus.

I4. L'optique au XVII ${ }^{\mathrm{e}}$ siècle. - L'aurore du xvit sickle avait vu natre l'oplique scientificpere el prende corpsi l'optique appliquée. Dès lors, grâce au concours des recherches théoriques, des observations, des expériences, lopliçue entiore prorrcessera rapidement. Ciest. l'époque oú l'arc-en-ciel trouse son explicalion à peu près définitive, avec Descartes et Huygens; où Gassendi énonce. sans trop de peruves toutelois, l'athaiblissenent de l'intensité lumineuse en raison du carré de la distance, et oú il altribue aux réfractions les couleurs de l'iris; où Grimaldi découve les phénonnènes de diffraction, et Bartholin ceux de la double réfraction du spatl, étudiés peu après par Huygens, qui, d'autre part, frappi des analogries entre les seusations lumineuses et sonores, suggère l'idée griniale des ondulations.

An point de rue des instruments proprement dits, signalons, à côté de perfectionnements secondaireś auxquels con- 
courent Huygens, Hévélius, Hooke, linvention du téleseope caloptrique ou à miroir par Mersenne, qui laissera la gloire de le réaliser à Jacques Gregory, et celle de le perfectionner a Newton ; celle du micromètre, à laquelle coopèrent Huygens, Malvasia, Auzout et Picard; celle de la lanterne nilagigue, due au Père Kircher.

I5̃. La découverte de l'achromatisme. - Gràce à lat loi de Descartes et au fonds d'idées légué par leurs devanciers, la conception d'un instrument optique devant satisfaire à des exigences données n'était qu'un jeu pour les subtils géomìtres qu'étaient les savants du vwni sićcle. On en rencontre la preuve évidente dans les ouvrages de l'épocue. Mais la réalisation des combinaisons projetées se heurtait invariablement à une difficulté qui paraissait insurmontable : les images produites manquaient de netleti. Ia cause tenait principalement aux irisations qui les bordaicnt.

Les astronomes, qui cóloient seulement les contingenecs kerestres, avaient tourué la difficulté en augmentant démesurément la longueur de leurs hunetles; la solution ne pouvait être généraliséc. Lne expérience de Now ton, mal conduite par excis de précaution, avait fait croire à la proportionnalité de la dévialion et de la dispersion des rayons non monochromatiques; la disparition des couleurs irisées paraissait, dies lors, aussi impossible que la quadrature du cercle. Lt cette conséquence imposait presque la substitution des surfaces réfléchissantes aux masses réfringrentes : solution médiocre à divers égards.

La conclusion que Dew ion avait cru pouvoir tirer de son expérience était heureusement inexacte. Des doutes furent émis par Euler, qui remarqua que l'œil humain, constitué uniquement par des corps réfringents, s̈il n'était pas absolument achromatique, jouissait d'un achromatisme enviable pour bien des instruments. Les tentatives d'Euler pour constituer des objectifs. - c'était un point capital, - sur Ie 
modèle de l'oeil ne furent pas couronnées de succès; mais l'idée était juste et féconde. Un opticien français établi en Angleterre, Dollond, l'utilisa et parvint à réaliser, en employant deux espèces de verres, les premiers objectifs achromatiques.

La difficulté ploysique était levée; la prévision des propriétés d'un système optique devenait du ressort de la géométrie; les profonds géomètres du $x$ rum siècle ne faillirent point à leur tàche : Clairaut, d'Alembert, Euler, plus tard Lagrange et Biot, suivis de savants d'ordre moindre, apporteront un concours précieux, inestimable, à l'étude des éléments optiques. C'est l'époque héroïque de l'optique instrumentale théorique. Ies savants qui édifièrent la merveilleuse analyse infinitésimale ne crurent pas déroger en s'astreignant, à propos de Iunettes, à des calculs élémentaires, fastidieux, prolixes et ingrats.

I 6. L'invention de la photographie. - Les recherches des géomètres, l'habileté des constructeurs avaient permis de tirer tous les fruits de la découverte de l'achromatisme. L'invention de la photographie suscita d'antres problèmes à résoudre, et, par contre-coup, força l'attention des opticiens à se reporter sur les instruments qu'ils avaient jugís parfaits.

L'étape fut d'ailleurs longue et laborieuse. Les premieres conclusions théoriques se heurtèrent à une difficulté matérielle : les verres, ni nombre fort limité, dont disposaient les constructeurs ne possédaient pas les propriétés préconisćes par le calcul. Les systèmes optiques réclamés par la praticue ne furent malgré. l'ingéniosité des opticiens, malgré leurs nombreuses tentatives expérimentales, obtenus d'abord qu'imparfaitement.

Pour réaliser les combinaisons suggérées par la théorie, il étnit indispensable de mettre à la disposition des opticiens des verres ayant des propriétés autres que colles des crowns et des flints employés jusque-là. Un maître verrier l'rançais, 
Guinand, avait, dès I 8/10, produit des variétés acceptables de matières convenables, mais dans des conditions onéreuses telles, que l'on avait dù s'arrèter devant la question économique. Le problème fut repris vers 1880 , en Allemagne; et, quelques années plus tard, les verres d'Iéna, - suivis d'ailleurs bientôt par des verres français au moins équivalents, faisaient leur apparition sur le marché. Il devenait dès lors possible de mettre à l'épreuve les théories élaborées par divers savants, parmi lesquels on peut regretter de ne pas rencontrer les successeurs qualifiés des grands géomètres du $\mathrm{xvII}^{\circ}$ siècle.

Ajoutons d'ailleurs que les perfectionmements apportés au travail du verre et au travail mécanique ont permis la réalisation de combinaisons jadis réputées inexécutahles, "n raison du degré de précision qu'elles exigeaiont.

\section{DIVISIONS DE LOUVRAGE}

7. - Les instruments d'observation préséntent un certain nombre de propriétés générales qui tiennent principalemont au mode de formation des images optiques. L'étude de ces caractères fera l'objet de la première parlie de l'ouvrage. Quelques lignes seront consacrées aux instruments de mesure.

Dans la seconde partic, on exposera l'organisation et le mode d'emploi des instruments, tant de mesure que d'observation. La matière sera traitée en suivant l'ordre indiqué plus hant (2).

Le cadre limité de ce volume ne nous a pas permis d'aborder les questions relatives au calcul, à la construction et à l'examen des instruments. On trouvera cependant, dans l'index bibliographique, un certain nombre de docunents qui s'y rapportent.

18. - Dans l'exposé qui va suivre, nous supposons con- 
nus les faits et les théories de l'Optique géométrique, los plénomènes essenticls de l'Optique plyysique. Ies notions el propositions nécessaires seront, s̈il y a lieu, énoncées en tenyss utile; les formules, rappelées à l'occasion sans dímonstrations, - le cas échéant, le mode de ces dernières ponrra ètre indiqué.

Des instruments qui pewvent être rangés dans la partie optique de la l'hysique générale; nous avons exclu ceux plus spécialement consacrés aux mesures optiques proprement dites; tels sont les photomed res, spectroscopes et spectromìtres, polariscopes et polarimètres, réfractometres, etc. Ces apparcils entrent parfois en jeu dans l'examen des instruments que nous considérons plus spécialement; des indications pouront être données sur eux, s’il est nécessaire; mais leur dule dinaillé aurait clendu dimesurement lo eadie de cot ouvrage. 


\section{INSTRUYENTS OPTIQUES}

\section{D'OBSERVATION ET DE MESURE}

\section{PREMIÈRE PARTIE \\ PROPRIÉTÉS GÉNÉRALES DES INSTRUMENTS}

\section{CIIAPITRE: I}

DES QUALTLS OPTIQUFS DES IYSTRL MEYTS.

I9. Les qualités qu'on doit rechercher, avant tout, dans les instruments d'observation se rapportent à la nettelé des images, à leur clarté, au champ de l'insl'unent et à la fidélité d'aspect des images.

Nous allons définir et préciser ces notions. Leur étude détailice fera l'objet de chapitres ultérieurs.

2o. Netteté des images; puissanco des instruments. - Puissance d'un instrument optique et netteté de l'image produite sont'des notions identiques 
qui ne diffèrent, dans Icur expression, qu'en raison du point de vue adopté : appréciation du róle de l'appareil, examen de la finesse de l'image.

Une longuc-vue est plus puissante qu'une autre si elle laisse distinguer des détails que la seconde ne peut discerner; par exemple, si elle permet à un observateur d'assurer qu'un individu tient les doigts d'uno main ouverts, tandis que le second appareil laisserait la question indécise. De deux microscopes, le premier est le plus puissant s'il laisse compter les stries do I'Amphipleura pellucida, lorsque le second ne montro qu'une nappe moutonnće. Les photographes apprécient la puissance de leurs objectifs, - leur finesse, disent les uns; leur bonté, prétendent les autres, d'après le degré de flon qui réunit les détails plus ou moins entrevus d'un paysage.

Quel que soit l'instrument, sa puissance est d'autant plus grande qu'est plus faible sur l'objet considéré l'intervalle minimum existant entre deux détails séparés sur l'image soumise à l'examen. La puissance de linstrument, corrélative de la nettetó des images, devient mesurable par son. pouwoir séparateur; d'aucuns disent encore son pouvoir pénélrant ou résolutif.

Cette facultó séparalrice prond une forme simple dans les observations astronomiques. Les distances angulaires des étoiles, - qu'il s'agisse de constellations ou d'itoiles doubles, - sont asse\% variées pour permettre de différencier deux lunetles ou deux télescopes de puissances presque égales : l'un des instruments montrera deux étoiles, lorsque l'autre n'en laissera voir qu'une. L'intérèt du fait tient ici à ce que les détails 
sur lesquels peut se baser l'évaluation du pouvoir séparateur se présentent sous la forme la plus simple : celle de points lumineux, les plus parfaits peut-être des points réalisables.

La puissance d'un instrument peul done se déduire, - exception faite des difficultés oxpérimentales, - de l'intervalle minimum, angulaire ou linéaire suivant le cas, qui sépare deux points lumineux dont les images définitives ne sont pas confondues. Pour les instruments destinés à la vision éloignée (lunettes, objectifs à paysages), le pouvoir séparateur s'évaluera en écarts angulaires (secondes sexagésimales, centigrades ou tangentes trigonométriques de ces angles); la puissance, qui doit logiquement ètre caractérisée par un nombre d'autant plus grand qu'elle est plus considćrable, se mesurcra par l'inverse de la tangente définissant le pouvoir séparateur. Ainsi une lunette séparant la seconde aura une puissance de 200 ooo. Pour les objectifs destinés aux reproductions, la même règle peut être admise; mais il doit être sous-entendu que la distance de l'objet est donnée, ou remplacée par la focale de l'objectif et le coefficient de réduction. Quant aux microscopes, il est d'usage d'évaluer à la fois le pouvoir séparateur et la puissance par le nombre maximum de lignes parallèles très fines, de stries, qui peuvent être discernées dans un intervalle de I millimètre.

21. Clarté des instruments. - Toutes les per sonnes qui se servent couramment de longues-vues n'ignorent pas que tel instrument, parfait en plein jour, devient presque inutilisable an crépuscule ou dès quo la brume envahit l'atmosphère; los images s'estompent et s'aflaiblissent; dans les mèmes conditions, une lunette 
de Galilée, de qualité moindre, pernet encore d'apprécier des détails ou de distinguer des objets dont la première laisse les formes indécises. La luminosité des objectifs photographiques s'évalue assé exactement par. le temps de pose qui, dans des circonstances identiques, donne aux clichés le même degré de vigueur. Les micrographes ont soin d'augmenter l'intensité de leur éclairage à mesure qu'ils élèvent les numéros de leurs objectifs.

La clarté des instruments, quelle que soit son origine, paraît être en liaison étroite avec celle des images produites, c'est-à-dire avec leur éclaircment superficiel; comme, d'ailleurs, ce dernier est nécessairement variable avec la clarté absolue de l'objet, il convient d'évaluer la clarté d'un instrument par le rapport des éclairements superficiels spécifiques de l'image et de l'objet, éclairements supposés unifornes. Bien que ce rapport, clarté propre de l'instrument, ne caractérise pas toujours entièrement à lui seul le degré de visibilité d'un objel. - on en verra plus loin les causes, sa considération est capitale dans la question actuelle. Les procédés photométriques habituels peuvent, avec les variantes convenables, être utilisés; il parait difficile de choisir une unité pour mesurer la qualité doni il s'agit.

22. Champ d'un instrument. - Ln instrument optique, - de position et. si ses éléments sont mobiles, de configuration données. - ne permet de voir netlement que les objets situés dans une certaine région de l'espace. Cette région constitue le champ de l'instrument, - le champ réel, précise-t-on habituellement. 
Ce champ est un solide, au sens géométrique du mot. La qualité que possèdent les instruments d'ètre en général des systèmes optiques centrés, et par suite de posséder uı axe de révolution, impose à l'une des surfaces limites du champ d'ètre un cône de révolution autour de cet axe. C'est ce cône qui circonscrit le champ angulaire de l'appareil.

Mais, des objets situés dans le champ angulaire, seuls donnent des images nettes ceux qui sont compris entre deux autres surfaces, également de révolution autour de l'axe du système, et dont la forme est plus ou moins voisine d'un plan ou d'une sphère. Elles limitent le champ en profondeur de l'instrument.

Le champ récl, total, doit donc être défini au moins pal cleux nombres. L'un caractérisera le champ angulaire : ce sera l'angle au sommet du cône, évalué soit en unités d'angle (degrés, grades et leurs fractions). soit par sa tangente trigopométrique, - surtout pour un champ ne dépassant pas $6^{\circ}$, - mise souvent sous la forme de la longueur en mètres du diamètre, à r ooo mitres de la section droite du cône. Cela s'applique aux instruments spéciaux pour l'observation à grande distance. Pour les objectifs photographiques, il est d'usage d'indiquer le champ par la désignation du format usuel de la plaque couverte, dans les conditions d'emploi de l'objectif; cette donnée, jointe à la valeur de la focale de l'organe, suffit pour la détermination du cône limite. Quant au microscope, c'est en spécifiant l'étendue linéaire maximun nettement visible d'un objet qu'on en évalue le champ : le millimètre est, dans ce cas, une unité en général convenable.

La seconde caractéristique se rapporte au champ en 
profondeur. Il suffit, dans la majorité des cas, qu'elle détermine les positions des points ou les deux surfaces terminales rencontrent l'axe de l'instrument. La distance de ces points est la profondeur du champ. L'unité peut, suivant la diversité des conditions, être le kilomètre, le mètre, lo centimètre; pour les microscopes, il faul des fractions, généralement faibles, du millimètre.

Les valeurs des éléments qui mesurent le champ n'oflent d'ailleurs de garanties qu'autant qu'on a défini la netteté et la clarté exigées pour les images : car tout se tient en optique. L'organisation mécanique d'un instrument, - l'emplacement et l'ouverture des diaphragmes, par exemple, - peut attribuer un champ angulaire considérable à un instrument; mais si, vers les bords du champ, les images sont brouillées ou leur clarté insuffisante, il convieut de réduire impitoyablement les valeurs indiquées. Les donnćes relatives aux objectifs photographiques que l'on rencontre dans les prix-courants sont trop souvent dans ce cas.

23. Fidélité d'aspect des images. - L'inage est une représentation fidèle de l'objet lorsqu'elle en conserve les colorations et la forme géométrique.

La première condition intéresse à un très haut degré la netteté des images; en la supposant réalisée, la question géométrique reste scule à examiner.

A ce point de vue, dans les instruments monoculaires, - Jes autres ne peuvent actuellement être envisagés, - la fidélité d'aspect exige que l'image puisse être considérée comme une perspective conique de l'objet. Cette condition comporte comme corollaire la 
similitude géométrique entre l'image et l'objet situé dans un plan normal à l'axe de l'instrument, le rapport de similitude pouvant d'ailleurs varier avec la position du plan objet. L'instrument est dit alors, suivant la terminologie allemande, orthoscopique ; dans le cas contraire, les images sont déformées; on exprime ce fait en disant, - terme d'originé anglaise, - qu'elles présentent de la distorsion. Un carré centré sur l'axe se transforme en quadrilatìre curviligrne dont les côtés se courbent vers l'axe ou dans la direction opposée; un système de circonférences équidistantes, situées dans un même plan et dont le centre commun est sur l'axe, donne, comme images, des circonférences ćgalement centrées sur l'axe, mais pour lesquelles le caraclèce d'équidistance n'est pas conservé. Le rapport entre les grandeurs, linéaires ou angulaires, des rayons correspondants de l'image et do l'objet, peut servir à évaluer, dans chaque région du champ, le degré de distorsion présenté par l'instrument; le rapport en question n'est constant que si l'appareil est orthoscopique.

24. Du grossissement dans les instruments. - Dans l'énumération des qualités essentielles d'un instrument d'observation, nous n'avons pas mentionné son grossissement; c'est pourtant cotte dernière caractéristique qui est, souvent, la première énoncée dès qu'il s'agit d'une longue-vue ou d'un microscope. L'opinion commune est qu'un instrument est d'autant plus remarquable que son grossissement est plus élevé. C'était aussi, mais avec une restriction qui la réduit à sa juste valcur, l'opinion d'Euler (24, lettre 88). "Plus une lunette grossif les objets, plus elle est sans doute parfaite, pourvu qu'aucune des autres bonnes qualités 
n'y manque. "Malheureusement ce n'est, trop souvent, qu'au détriment des autres propriétés de l'instrument, - qualités optiques, mécaniques, économiques, - qu'on en accroit lo grossissement : la perte est certaine, le bénéfice illusoire.

Il est aisć de donner à une excellente longue-vue un grossissement supérieur à celui qu'elle possède : il suffit de la munir d'un autre oculaire, plus fort, suivant le terme admis. Les images sont amplifices; l'intervalle qui séparait deux détails de l'inage s'élargit; mais il est exceptionnel que des détails nouveaux deviennent perceptibles; la puissance maximum de l'iustrument ne subit aucune variation. En retour, la clarlé s'abaisse nécessairement, et souvent le champ se rétrécit.

On sait aussi que cette diminution du champ est corrélative de l'accroissement du grossissement obtenu, dans les microscopes, par le changement de l'objectif ; mais, si la puissance est ici généralement augmentée, la réduction du champ peut être telle que l'observation perd toute valcur : les arbres empèchent de voir la forêt.

L'emploi de forts grossissements n'a en lui-même d'utilité que dans des cas exceptionnels, plus rares dans les instruments d'observation que dans ceux de mesure. Pour ces derniers, on peut citer l'exemple de la lunette méridienne. Dans les autres, un fort oculaire peut arriver à faire discerner deux détails ténus mal séparés par l'objectif, en affaiblissant suffisamment la zone commune à leurs taches centrales; en dehors de ce cas, le rôle du grossissement parait se réduire à donner aux inages un aspect plus agréable pour certains 
observateurs ; mais ce caractère ne peut prétendre à être précisé.

Toutefois, dans les apparcils bien conçus, et dans des conditions d'emploi bien déterminées, la connaissance du grossissement, notion vulgaire et de facile compréhension, peut remplacer assez exactement celle. plus scientifique et plus délicate, de puissance. A ce titre, il est utile d'en faire mention dans la description d'un instrument.

25. Importance relative des qualités d'un instrument d'observation. - La question, journellement posée, est, dans sa généralité, insoluble. Chaque cas particulier doit ĉtre envisagé, et la réponse n'est pas du ressort exclusif de l'Optique.

A priori, il semble que la puissance d'un instrument constitue sa qualité primordiale. "La netteté dans l'expression, - dit Euler (24, lettre 8I), qu'on peut toujours citer en pareille matière, - est un article si important entre les qualités d'une lunette, qu'il semble l'emporter sur tous les autres..., puisque tout le monde convient qu'une lunette qui ne représente pas nettement les images des objets est fort défectueuse. " L'opinion est, en effet, unanime; les divergences ne s'accusent qu'au moment de déterminer le degré de netteté convenable. Tel instrument très puissant peut n'ètre qu'un médiocre outil, si la netteté des images n'a été obtenue qu'en donnant au champ une amplitude trop faible.

En général, ce sont les conditions normales d'emploi de l'instrument qui fourniront la base d'appréciation des qualités exigibles. Pour un type donné, les qualités seront, dans des degrís limités, l'une indispensable

Instruments optiques. 
à réaliser, l'autre utile; quant aux dernières, il sera avantageux de les obtenir, autant que le permettront et les conditions pratiques - en général, mécaniques - d'emploi, et le prix de revient de l'appareil. Ainsi une longrue-vue d'exploration devra avant tout posséder un grand champ; en second lieu, avoir une clarté suffisante; la netteté ne viendra qu'en troisième ligne; la distorsion des images pourra être négligée. Au contraire, un objectif destiné à des travaux cartographiques devra fournir avant tout des images exemptes de distorsion; ensuite donner une netteté suffisante. Les exemples pourraient ètre multipliés à l'infini.

Le mode d'emploi d'un instrument inpose à ses qualités optiques, mécaniques, économiques, des conditions, non pas contradictoires, mais incompatibles. Le géomètre pourrait se borner à constater le fait; le praticien doit passer outre, et, après discussion des sacrifices à consentir, choisir la plus plausible, à son sens, des solutions acceptables. Les qualités optiques d'un instrument sont done le résultat d'un compromis nécessaire. Il serait injuste de l'oublier quand on apprécie une combinaison optique.

26. Qualités des instruments de mesure. Il ne saurait être question de produire, ici, J'énumération des qualités optiques nécessaires aux appareils de mesure; car autant d'instruments, autant de problèmes.

Deux remarques générales, seulement, semblent utiles.

Dans ces instruments, la mesure est le but; l'optique est le moyen, et partant, l'accessoire. Par suite, il ne faut pas hésiter à se contenter, si elles sont suffisantes, de qualités optiques en apparence médiocres 
pour un instrument d'observation. C'est ainsi, par exemple, qu'on pourra parfois se borner à obtenir dans une lunette la netteté maximum au centre, sans s'occuper outre mesure de ce qu'elle devient sur les bords du champ.

La seconde remarque a trait à la précision de l'appareil. L'usage auquel on destine celui-ci détermine le degré de précision de la mesure; un sage équilibre doit régner entre les divers éléments de l'instrument; chacun d'eux doit concourir pour sa part, sans excès, à la précision définitive. Il peut être mauvais de trop soigner certains organes, de les travailler ou de les monter avec une précision propre que ne comporte pas celle de l'appareil; car toute prócision se paye, et tonjours fort cher, et sa conservation exige des soins minuticux. 


\section{CHAPITRF II}

HORE DE L'OELL DANS LA VISION INSTRLMENTALF

37. Nécessité de cette étude. - La connaissance des propriétés de l'wil est indispensable pour l'élude des instruments optiques. Lassertion pourrait passer pour une évidence ou une naiveté. Il faut en expliquer la portée.

Dans l'étude d'un phénomene mécanique, thermique, électrique, acoustique même, ce n'est pas, le plus souvent, l'organe humain qui a pu le déceler qui est utilisé dans l'observation ou la mesure de ce pliénomine. Fntre le siege de ce dernier et l'expérimentateur, s'intercale un appareil special, - d'exploration ou de mesure, - dont les déformations sont fonctions de la nature et de la grandeur du phénomène, et qui sont perçues par un organe déterminé, l'œil généralement. Ic roltmètre indique la tension d'un réseau sans que l'opérateur en ressente les effets; un observateur sourd peut, en examinant le diagramme tracé sur le papier enfumé, indiquer, mieux que le meilleur des musiciens, la note ćmise.

Les phénomènes lumineux ne peuvent, au contraire, être étudiés qu'arec le concours de l'organe mème qui les perçoit. De là, suivant les observateurs, des divergences qui naissent dis qu'il sagit de questions touchant à la netteté, à la clarté ou à l'appréciation de certaines conséquences géo- 
métriques et physiques de la vision. Ein particulier, l'examen et le contrôle des instruments deviemnent délicats et donnent des résultats incertains, si chacun rapporte, sans corrections, ceux - ci à son ail.

Les notions qui ront suirre donneront les ćléments indispensables pour faire intervenir l'ceil dans la conception, I'organisation el la vérification des instruments optiques. Ia physiologie de l'oeil sera loin d'ètre épuisée. Sur ce sujet, Helmholtz a écrit un ouvrage remarquable (53) ct volumineux, auquel nous emprunterons beaucoup.

28. L'œil idéal. - Autant d'honmes, autant d'yeux; ce dernier nombre pourrait même être large ment augmenté, car beaucoup d'ètres humains n'ont pas leurs yeux identiques. De cette diversité d'organes, la Science doit en former un type, afin de pouvoir en soumettre les propriétés aux règles de la Logique. Cet oil, de caractère un peu théorique, possédera des propriétés moyennes (au sens vulgaire de qualificatif); il aura les caractéres que l'on rencontre dans ce qu'on appelle habituellement les bons yeux, organes sains, exercés à la vision normale dans les circonstances variées.

Nous l'appellerons l'ceil idéal; le mot idéal n'est pas pris ici dans le sens de parfait.

I.es propriétés des instruments étant établies pour cet organe abstrait, il sera facile ensuite d'en déduire celles qui se rapportent à des yeux naturels, normaux ou peu anormaux. Il ne s'agira plus alors que de variations de quelques grandeurs; et l'on sait que, dans des cas très nombreux, la règle de proportion suffit aux calculs.

29. Éléments optiques de l'œil. - Les élé- 
ments réfringents de l'oil sont, en allant d'avant en arrière :

I $^{\circ}$ La cornée transparente, lame solide, mince, à faces sensiblement parallèles et convexes vers I'extérieur. Sa forme est assez exactement celle d'une calotte d'ellipsoïcle, pas tout à fait de révolution, dont le sommet est au centre des faces.

$2^{\prime \prime}$ L'humeur aqueuse, liquide incolore, constitué par de l'eau tenant en solution ou suspension $2 \%$ de matières solides.

$3^{\circ}$ Le cristallin, corps lenticulaire, biconvexe, dont la face antérieure est la plus aplatie; c'est une masse gélatineuse, élastique, plus consistante au noyau ou centre, et composée de couches dont l'indice de réfraction augmente de l'extérieur à l'intérieur. Les surfaces terminales appartiennent à des ellipsoïdes de révolution.

$4^{\circ}$ Le corps vitré, contenu dans la membrane hyaloïde, est, comme le cristallin, ure masse gélatineuse, mais de nature moins consistante et de constitution différente; elle est formée d'eau et, dans la proportion totale de $2^{0} / 0$, de substances solides et d'une matière mucilagineuse.

L'indice du corps vitré est un peu plus élevé que celui de l'humeur aqueuse; tous deux, supérieurs à celui de l'ear , en different à peine.

Un diaphragme, l'iris, esi placé en avant et contre le cristallin; son ouverture circulaire, la pupille, on disait jadis la prunelle, - varie de diamètre, entre 2 et ro millimètres environ, suivant l'intensité de la lumière ambiante.

3o. CEil schématique de Listing; œil réduit. 
- Les caractéristiques de l'œeil schématique, organe théorique, sont les suivantes (fig. I) :

Les surfaces des éléments réfringents sont supposées sphériques et rigoureusement centrées (dans l'exil réel, le centrage n’est pas parfait); la cornće C est réduite à une surface gćométrique; I'humeur aqueuse et le corps vitré sont considérćs commo identiques; le cristallin I'T" est supposé homogène.

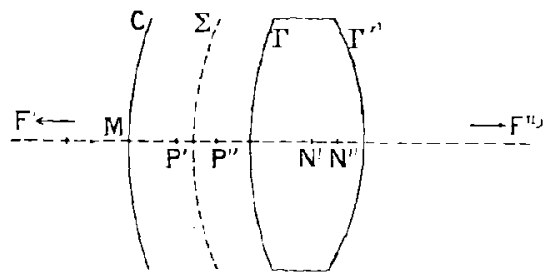

Fig. 1.

Les données numériques adnises sont :

Rayons de courbure : de la cornée, 8 millimètres; de la face antérieure du cristallin, Io millimètres; de sa face postérieure, 6 millimètres;

Épaisseurs suivant l'axe : de l'humeur aqueuse, 4 millimètres; du cristallin, 4 millimètres; du corps vitré, 15 millimètres environ.

Indices de réfraction : du cristallin, I,455; de l'humeur aqueuse et du corps vitré, 1,337 .

L'axe du système est dit axe de l'ail ou axe optique.

Le calcul permet de déduire de ces hypothèses les résultats ci-après :

Posilions des points principaux :

$$
\mathrm{MP}^{\prime}=2^{\mathrm{mm}}, \mathrm{I} 7^{5} ; \quad \mathrm{MP}^{\prime \prime}=2^{\mathrm{mm}}, 57^{\mathrm{a}} ;
$$


Positions des points nodaux :

$$
\mathrm{MN}^{\prime}=7^{\mathrm{mm}}, 24_{2} ; \quad \mathrm{MS}^{\prime \prime}=7^{\mathrm{rum}}, 640 ;
$$

Positions des points foyers :

$$
\mathrm{MF}^{\prime}=7^{\mathrm{mm}} \cdot 765 ; \quad \mathrm{MF}^{\prime \prime}=27^{\mathrm{mm}}, 7^{15} ;
$$

et par suite :

Focale antérieure,

$$
\mathrm{P}^{\prime} \mathrm{F}^{\prime}==15^{\mathrm{mm}}, 007 .
$$

Focale postérieure,

$$
\mathrm{P}^{\prime \prime} \mathbf{F}^{\prime \prime}=20^{\mathrm{mm}}, 075
$$

Intervalle des points principaux,

$$
\mathrm{P}^{\prime} \mathbf{P}^{\prime \prime}=\mathrm{o}^{\mathrm{mm}}, \mathbf{3}_{9}{ }^{8}
$$

Intervalle des points nodaux,

$$
\mathbf{N}^{\prime} \mathbf{N}^{\prime \prime}=o^{\mathrm{mm}}, 39^{8} \text {. }
$$

En supposant réunis, vu leur faible intervalle commun, d'une part les points principaux et de l'autre les points nodaux, on peut remplacer tout le systeme réfringent de l'œil par une surface unique \' séparant l'air d'un milieu réfringent, dit ceil réduit, d'indice égal à celui de l'humeur aqueuse. Cette surface, sphérique, passe par le point principal unique, a pour centre le point nodal, et son rayon est égal à $5^{\mathrm{mm}}, 125$.

3r. Formation des images; rétine. - Les images des objets extérieurs se peignent, réelles et renversées, sur une surface organisée, concave vers l'intérieur de l'oeil, la rétine, qui est un épanouissement du nerf optique et qui limite, en arrière, le corps vitré. La rétine est constiluée par les éléments nerveux ordinaires, et par d'autres éléments spéciaux, filiformes, très réfringents, effilés à leurs bases, qui pénétrent. 
dans la couche sous-jacente, leurs extrémités libres formant la surface même de la rétine à laquelle ils sont normaux. Ces éléments. pressés les uns contre les autres, sont de deux sortes : des bâtonnets (bacilli) cylindriques, de 65 a 80 ph de longueur et de $\mathrm{I}, 8$ p. de diamètre; des cônes (coni), de 15 à $20 \mu$ de longueur et de 4 à 5 p. de section à la surface de la rétine, prolongés d'ailleurs dans les tissus par des bâtonnets spéciaux de cône.

Ces éléments, et plus particulièrement les cônes, paraissent être les récepteurs utiles des sensations lumineuses. Leur répartition est inégale sur la surface de la rétine. Dans une région circulaire de I à 2 millimètres de diamètre, la lache jaune (macula lutea retinx), dont le centre est à $\mathrm{I}^{\mathrm{m} m}, 5$ environ du pied de l'axe optique et vers le côté temporal, les cônes sont plus nombreux que les bâtonnets; ceux-ci disparaissent même au centre de la tache, où se trouve une dépression, la fovea centralis, de $\mathrm{O}^{\mathrm{mm}}, 2$ de diamètre, pavée de cônes moins épais, 2 à 3 y. A mesure qu'on s'éloigne de la tache jaune, la proportion des bâtonnets augmente; exception faite du punclum cæcum, entrée du nerf optique, presque symétrique de la tache jaune par rapport à l'axe de l'œil, où disparaissent cônes et bâtonnets.

32. Orientation de l'œil dans la vision nette; centre de rotation. - La faculté de perception de la rétine, nulle à l'entrée du nerf optique, maximum dans la fovea centralis. très grande dans la tache jaune, diminue à mesure que l'imare rétinienne s'éloigne de cette dernière région. La sensibilité de la rétine semble liée à la présence des cônes. 
Dès que la volonté intervicnt pour regarder un objet, l'ocil, par suite d'une habitude inconsciemment acquise, s'oriente de façon que le point particulièrement fixé forme son image au centre de la fovea centralis. Ce mouvement est accompli par le jeu automatique de six muscles moteurs. Le glohe oculaire ne pouvant avoir qu'un déplacement d'ensemble dans son orbite osseuse, ses déformations étant insensibles, tourne autour d'un point fixe par rapport à la masse cranienne, ef fixe, par suite, par rapport à lui. Ce point, dit centre de rolation de l'ceil, est situé sur l'axe optique à $13^{\mathrm{mm}}, 6$ en moyenne en arrière du sommet de la cornée. Pour les yeux myopes, cette distance doit être portéc à près de $\mathbf{r} 6$ millimètres, et réduite à $\mathbf{I} 2^{\mathrm{vmm}}, 3$ pour les yeux presbytes.

La, ligne qui joint au centre de la fovea le point nodal postérieur de l'oil constitue la partie postérieure de la ligne visuelle; sa partie antérieure, qui Jui est parallèle, joint, dans le cas de la vision nette, le premier point nodal au point fixé.

33. Champ de la vision. - L'œil immobile ne perçoit très nettement que les détails angulairement peu éloignés de la ligne visuelle; ce champ de la vision nette ne paraît pas supérieur à un demi-degré; cette valeur correspond asser exactement à l'angle sous-tendu du second point nodal par la fovea centralis, et aussi à l'étendue visible des caractères d'écriture à la distance habituelle de la lecture ( 3 millimètres à 30 centimètres).

Yais, en raison de l'extrème mobilité de l'œil autour du centre de rotation et de la rapidité de transmission au cerveau des impressions rétiniennes, le champ de la vision pratique est de beaucoup plus étendu : on peut 
appeler ainsi le champ que, sans fatigue et sans action consciente de la volonté, l'oeil peut parcourir.

L'oeil, au repos, a dans l'organisme humain une position telle que la ligne visuelle est, à peu près, parallèle au plan de symétrie et perpendiculaire à l'axe du corps (la verticale, l'homme étant supposé debout). Le champ de la vision pratique est un còne à base elliptique dont l'axe horizontal a une étendue angulaire de $30^{\circ}$ et l'axe vertical de $20^{\circ}$. Sous l'action de la volonté, ces nombres peuvent être multipliés par 2 et mème par 3 .

Les champs ainsi définis se rapportent à la vision directe; tandis que se forme sur la tache jaune l'image nette du point visé, les autres régrions de la rétine perçoivent, moins distinctement, mais dans des conditions souvent suffisantes, les objets extérieurs au champ do la vision nette; - c'est ainsi qu'en regardant attentivement un point tout particulier d'un paysage, on ne perd pas de vue les allées et venues des personnes autour de soi. Le champ ainsi défini, champ de la vision indirecte, a une amplitude considérable : elle atteint $150^{\circ}$ dans le sens horizontal, $120^{\circ}$ dans le sens vertical.

34. Netteté des images rétiniennes; accommodation. - La simple orientation de l'œil suffit à la netteté de la vision lorsqu'il s'agit d'objets éloignés; car l'œil normal au repos est disposé pour la vue à l'infini, les images se formant alors sur la rétine. Lorsque l'objet se rapproche, la perception ne devient nette qu'à la suite d'une modification automatique de l'ceil, qui porte le nom d'accommodation, et qui a pour but de placer l'image sur la surface rétinienne.

La modification en question paraît seulement con- 
sister dans un chancrement de forme du cristallin, qui devient ainsi une lentille à foyer variable : les deux surfaces de l'élément augmentent de courbure, le sommet de la face postérieure restant en place. Accessoirement, la pupille se resserre.

Les variations dont il s'agit ne deviemnent sensibles qu'au momont où le point visé est parvenu à i 5 ou 2o mètres; elles se continuent jusquau moment où l'objet arrive à la distance, 25 centimètres environ, du punctum proximum. La forme du cristallin ne variant plus, si l'objet se rapproche encore, son image devient de plus en plus confuse.

Dans l'cil schématique de Listing, on attribue l'accommodation à la variation de courbure de la face postérieure du cristallin (qui passe de 6 à $5^{\mathrm{nn}}, 5$ ) et au changement d'épaisseur du nême élément (qui augmente de $\left.o^{\mathrm{mm}}, 4\right)$, — l'objet se déplaçant de l’infini à I 3 centimètres, punctum proximum encore admissible pour cortaines vues flexibles et excrcées. Ces hypothèses conduisent à un déplacement de 2 millimètres, en avant, du foyer principal postérieur de l'ceil, et à une diminution de ro millimètres de la focale propre du cristallin.

35. Perception des détails par l'œil. Acuité visuelle; loi de Hooke. - Les propriétés spéciales de l'cil qui règlent son accommodation semblent pouvoir être condensées dans les deux faits suivants, assez étroitement liés entre eux :

I" In point géométrique lumineux et une petite aire lumineuse donnent la même impression rótinienne, - celle d'un point lumineux physique, comme nous l'appellerons, - quand l'angle sous-tendu du point nodal 
antérieur par la dimension linéaire maximum de l'aire radiante est au plus égal à une minute.

Il faut supposer que le plan de l'aire, normal à la ligne visuelle, est plus loin que le punctum proximum ; que les intensités totales dı point et de la surface sont sensiblement équivalentes, et qu'en outre, en raison des phénomènes d'irradiation, l'intensité lumineuse n'est pas trop forte.

$2^{\circ}$ Deux points géométriques lumineux sont vus distinclement séparés lorsque leur distance angulaire, le point nodal antérieur de l'œil étant au sommet, est au moins égale à une minute.

Des restrictions analogues à celles indiquées plus haut doivent être faites.

On peut donner à l'ensemble de ces deux propriétés le nom de loi de Hooke, - plus spécialement attribué à la seconde. La grandeur, une minute, qui entre dans leur énoncé porte le nom d'acuité visuelle; elle n'est autre que la mesure du pouvoir séparateur de l'wil considéré comme instrument optique. Sa puissance est dès lors 3 ooo.

La valeur d'une minute admise pour l'acuité visuelle a été indiquée par Hooke à la suite d'observations de couples d'étoiles. Des valeurs différentes, généralement plus élevées, ont été indiquées par d'autres expérimentateurs opérant dans d'autres conditions. Le nombre de Hooke semble le plus admissible; il est habituellement adopté.

36. Profondeur du champ de la vision. Pour des faisceaux déliés, l'œil peut ètre considéré comme aplanétique. L'organe accommodé donne alors d'un point lumineux une image ponctuelle rétinienne.

Instıuments optiques. 
$\mathrm{Si}$, sans variation d'accommodation, le point lumineux se déplace, l'image rétinienne devient une petite aire sensiblement circulaire, qui continuera à donner une sensation ponctuelle tant qu'elle sera vue du second point nodal sous un angle inférieur à une minute; quand cette limite sera atteinte, le diamètre de la tache sera de $5 y$. Ce fait peut être la conséquence du déplacement du point objet soit en deça soit an delà de sa position initiale. La distance entre les deux positions extrèmes est la profondeur du champ pour l'état d'accommodation donnée. Dans cet intervalle, tout point géométrique lumineux donne conme image un point physique, l'accommodation de l'ceil élant invariable. Le calcul des systemes optiques permet d'évaluer la profondeur du champ. En particulier, pour l'œil au repos, c. a. d. accommodé à l'infini, l'objet peut se déplacer jusqu'à 15 mètres environ avant que le diamètre de son image atteigne $5 \%$; ]a pupille est, ici, supposée ouverte à 4 millimètres do diamètre. Sous une autre forme, on peut dire que, pour l'ceil, l'infini commence vers rà mètres.

$3_{7}$. Profondeur de foyer de l'œil. - Dans les mêmes conditions, le degré d'accommodation de l'œil est indifférent, pour la netteté des images, pourvu que la rétine coupe l'une ou l'autre des nappes du cône des rayons réfractés suivant un cercle de diamètre inférieur à 5 u. L'intervalle des deux sections satisfaisant à celte condition est la profondeur de foyer de l'œil. Pour un objet situé à l'infini, cette caractéristique est d'environ $50 \mu$.

38. Image solide d'un point. - L'image d'un point est donc, à peu près indifféremment, l'une ou 
l'autre des sections d'un còne à deux nappes, limité. Ce petit volume peut être appelé l'image solide du point. Sa figure exacte étant détermince pour une accommodation donnée, on obtiendra l'image géométrique sur la rétine, pour une accommodation différente, en déplaçant ce solide suivant son axe. Ce glissement sera accompagné d'une extension d'autant plus faible que le seront les variations de forme du cristallin. La variation de position du point lumincux entrâne également un mouvement de limage solide.

En réalité, l'image solide sera un pseudo-còne, c'est a dire uno sorte d'hyperboloide de révolution très allongé; cela, en raison du non-aplanétisme absolu de l'œil; mais le cercle de gorge ne parait pas avoir un diamètre supérieur à quelques dixièmes de micron.

39. Clarté des images rétiniennes. - Cette clarté est, dans une certaine mesure, assurée par le jeu involontaire de l'iris. Dès que l'intensité de l'objet augmente, les actions réflexes provoquées par l'excitation anormale de la rétine amènent le rétrécissement rapide de la pupille; si son ouverture minimum, 2 millimètres environ, est insuffisante pour faire tomber au taux convenable l'éclairement spécifique de l'image, la persistance de la fixation est suivie de troubles visuels, à moins que les cils et les paupières n'arrêtent à la fois clartó et netteté. Quand, au contraire, l'intensité de la lumière reçue s'abaisse, la pupille s'ouvre lentement de manière à accroître l'éclairement de l'image. La valeur de sa grandeur maximum, 8 à ro millimètres, paraît tenir à l'apparition des aberrations, comme sa valeur minimum semble être limitée par les phénomènes de diffraction. 
L'ouverture normale de la pupille est de 4 millimètres. C'est celle qui convient aux éclairages moyens : objets à l'ombre, en plein air, par un beau soleil et une atmosphère calme et pure. Il est difficile de préciser autrement dans celte appréciation.

40. Sensibilité de l'œil aux diverses radiations. - $W$. Herschel a essayé de déterminer la sensibilité en question en cherchant la distance maximum à laquelle étaient perceptibles des caractères d'imprimerie éclairés par des sources de diverses couleurs. Le procédé lui a indiqué la couleur jaune légèrement orangée conme étant la plus efficace; le vert viendrait ensuite. En s'éloignant de part et d'autre de ces couleurs voisines dans le spectre, la sensibilité de l'weil diminuerait rapidement. Les nombres du tableau ci-après indiquent (colonne $\mathrm{S}$ ) avec une approximation suffisante le degré de sensibilité de l'œil.

\begin{tabular}{|c|c|}
\hline Rougre (raie B) & $\mathrm{s}=$ \\
\hline Orangé (raie C) & 20 \\
\hline Jaune (raie D) & 100 \\
\hline Vert (raie E) & 50 \\
\hline Bleu (raic $F$ ) & 7 \\
\hline Indigo (raie G) & 0,5 \\
\hline Violet (raie H) & $0, \mathbf{I}$ \\
\hline
\end{tabular}

Lá seconde colonne (L) mentionne la proportion de la couleur qui existe dans la Iunière blanche solaire. La notion est peu précise; elle parait dériver de l'expérience classique du disque de Newton.

41. Valeur photométrique de l'œil. - L'ceil apprécie mal le rapport des intensités de deux sources on celui des éclairements de deux surfaces sauf lorsque 
ce rapport est très voisin de l'unité, anquel cas la valeur qui lui est attribuée est $\mathbf{I}$. Il est indispensable d'ailleurs que la lumière soit donce (ni trop vive, ni trop faible en intensité absolue) et que les couleurs soient identiques. - Pour des colorations différentes, le problème photométrique toujours posé, souvent résolu sans succès, n’a pas un sens délerminé.

En lumière blanche, on légèrement jaunàtre, l'œil ne percoit pas de différence entre deux éclairements Iorsque leur rapport differe de l'unité de $\frac{I}{6 / 4}$ au plus. Cette propriété est une conséquence de l'observalion photométrique de Bouguer, qui a constaté qu'une. impression lumineuse disparaissail lorsqu'elle se superposait à une autre $6 f_{1}$ fois plus forte.

La sensibilité de l'cil peut être accrue par la mobilité de l'une des taches éclairées par rapport à l'autre (Arago), et, s'il s'agit d'une source punctiforme peu intense sur fond plus obwur, en amenant l'inage de la source un peu en dehors de la tache jaune, c'est-àdire daus une région plus sensible parce qu'elle travaille moins.

42. Chromatisme de l'œil. - Bien que l'cil soit moins achromatique qu'il n'est aplanétique, la substitution de la lumière blanche, solaire, à une source monochromatique (jaune ou vert francs lumineux) ne paraît pas diminuer la netteté des objets éclairés.

Le chromalisme de l'oxil, jadis nié, n'est pas douteux. L'observation de Fraunhofer (mise au point de l'oil sur le réticule d'un spectroscope), l'expérience des cours agités de Wollaston en domment la preuve. La 
remarque suivante a le même caractère : si, à une distance de 4 ou 5 metres, on regarde une affiche rouge franc bordée d'une large bande vert foncé, le cadre paraît faire une saillie bien accusée. Le fait s'explique par la position du foyer des rayons verts en avant de celui des rayons rouges.

L'observation de Fraunhofer conduit a admettre $0: 4$ à 0,6 millimètre pour la distance entre les foyers des rayons extrêmes visibles rouges et violets. En adoptant la valeur de 0,5 millimètre, les données attribuées à l'ceil réduit de Tisting permettent de calculer le diamètre du cercle chromatique, la rétine étant accommodée pour les rayons moyens. Avec une pupille de 4 millimètres d'ouverture, " diamètre moyen de la pupille d'yeux normaux. " dit Helmholt, le diamétre du cercle de diffusion est de 5o $\psi$, soit dix fois celui de la tache maximum qui constitue un point image physique. Si cependant la sensation ponctuelle se conselve, c'est que, en dehors du centre où se condensent les rayons verts et jaunes. les autres couleurs du spectre ne possedent plus une intensité suffisante pour impressionner efficacoment la rétino.

43. Vision binoculaire; convergence optique. - Les propriétés précédentes concernent la vision monoculaire; à la vision binoculaire s'en ajoutent d'autres.

Dès que le regrard fixe un point. chaque wil s'accommode séparément pour la distance de l'objet; mais, en même temps, les yeux se disposent de telle façon que leurs lignes visuelles concourent sur le point fixé. Chacune des deux images de celui-ci se forme, dans le cas maximum de netteté, au centre de la fovea correspondante; les images des points voisins se peignent 
autour, en des points correspondants des deux rétines.

Aussi bien que pour l'acconmodation, les yeux sont, au repos, disposés pour la vision binoculaire à l'infini : les lignes visuelles sont parallèles. Quand l'objet examiné se rapproche, la convergence de ces liognes croît de $0^{\circ}$ jusqu'à $\mathbf{\text { ō }}{ }^{\circ}$ environ, correspondant à un punctum proximum de 25 centimètres, et à un ćcart noyen des centres de rotation de 65 millimètres.

\section{Facultè stéréoscopique de l'œil ; percep- tion de la profondeur. - Loi de Helmholtz. -} L'accommodation et la convergence sont fonctions de la distance de l'objet à l'observateur. Les variations de l'accommodation sont assez mal perçues et ne peuvent être utilisées pour apprécier les variations de distance de l'objel; il en est autrement des variations de la convergence.

Si deux points lumineux A et B (fjg. 2) sont à peu près dans la niême direction que le regard, les youx étant en $O$ et $O^{\prime}$, l'observateur pourra juger de la situation relative en profondeur des deux points dès que la différence des parallaxes

$$
x=\mathrm{OAO}^{\prime}, \quad \beta=\mathrm{OBO}^{\prime}
$$

atteindra une valeur suffisanto pour donner à cet observateur la sensation d'une variation de convergence. Helmholtz a indiqué, sous la forme suivante, la valeur

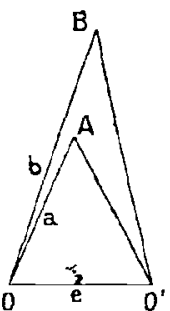

Fig. 2. d'une minute : "La comparaison des images rétiniennes des deux yeux, pour la vision stéréoscopique, se fait avec la même exactitude que l'appréciation des plus petites distances dans un seul et mếme acil. " 
En appelant $a$ et $b$ les distances $\mathrm{OA}, \mathrm{OB}$ supposées assez grandes par rapport à l'écartement oculaire $\mathrm{OO}^{\prime}=e$, la vision binoculaire pourra juger que $\mathrm{B}$ est plus éloigné que $A$, quand on aura :

$$
\frac{e}{a}-\frac{e}{b} \geq \frac{1}{3000} \text {; }
$$

$\frac{\mathrm{r}}{3000}$ est la tangente trigonométrique de $\mathbf{r}^{\prime}$.

En appelant $\sigma=b-a$ la différence de profondeur, supposant $e=65$ millinètres, et arrondissant le produit 3 ooo $e$ à 200 mètres, la formule précédente donne pour la profondeur minimum perceptible, - l'unité de mesure étant le mètre :

$$
\pi=\frac{a^{2}}{200-a} \text {. }
$$

La loi reste valable quand les points lumineux sont remplacés par des objets, pourvu qu'on n'ait sur eux aucun renseignement autre que celui qui peut résulter de la variation de convergence. Elle devient inutile si l'objet rapproché.cache plus ou moins l'objet éloigné ou projette de l'ombre sur lui, si, dans le jugement, on tient compte de la grandeur, exacte ou non, des objets, etc. Ainsi la relation précédente implique, par la valeur infinie que prend $\sigma$ pour $a=200$ mètres, l'impossibilité d'assurer, par la vision seule, qu'un homme placé à plusieurs kilomètres est plus éloigné qu'un autre situé à 200 mèlres; on ne se trompe pourtant guère, pratiquenent, lorsque lo premier est $\dot{a}$ 300 mètres seulement. C'est que les statures sont généralement comparables; la conclusion pourrait être erronée si l'homme le plus rapproché était un nain vis-à-vis de l'autre. 
La loi d'Helmholtz, généralement acceptée, ne parait pas avoir été systématiquement vérifiée pour des valeurs très diverses de la distance. Son auteur l'a déduite d'expériences de laboratoire, parfaites comme méthode, mais sans envergure. Elle semble cependant, d'après quelques-unes de ses conséquences, pouvoir ètre admise.

- La vision binoculaire offre encore l'avantage de permettre une appréciation plus exacte de la forme des objets,s, soit par la perception de leur profondeur, soit par la différence géomótrique des images produites sur chaque rétine. Ia sensation cérébrale esl toujours unique; mais l'image n'est plus une simple perspective sur un seul tableau; elle résulte de la fusion de deux pareilles figures, les points de vue étant différents et simultanés : c'est cette dernière condition qui définit la vision sléréoscopique.

45. - Les considérations précédentes, applicables aux yeur normaux, ou.emmétropes, ne convicnnent qu'avec les restrictions nécessaires aux yeux, amétropes (brachymétropes ou myopes, hypermétropes ou presbytes), ainsi qu'aux yeux astigmates, et aux organes dont la sensibilité est émoussée.

46. Rôle de la plaque photographique. - Entre l'wil et l'objectif photographique s'interpose habituellement la plaque sensible. La connaissance de ses propriétés générales est nécessaire; son étude détaillée sort du cadre de cet ouvrage.

La plaque sensible reçoit l'image réelle; les manipulations ultérieures, d'ordrc chimique, matérialisent celle-ci (étendant le sens du mot, nous les désignerons sous le terme de développement de l'image). 
Si absolue que soit la convergence des rayons formant un point de l'image réelle, ce point fưt-il d'ailleurs exactement sur la couche sensible (mise au point parfaite), celle-ci sera impressionnée, non en un point géonétrique, mais sur une petite surface plus ou moins étendue. Le diamètre de cetto aire, à peu prés circulaire, dépendra de la nature de la couche, et, plus spécialement, -_ en l'état actuel de l'organisation des plaques, - de la constitution du support (collodion, gélaline, etc.). Les opérations du développennent révéleront l'inage qui se présentera sous la forme d'un point noir - la production d'un négatif est le cas normal - de dimensions finies. Quand les points objets seront trop rapprochés, lours images formeront une aire sombre unique, continue, où olles ne paraitront plus séparées. La plarpue a donc, indépendamment de l'objectif supposé parfait, un pouvoir séparateur particulier.

Il n'est pas possible de lui assigner une valeur unicue. Le mode de développement, - où se révèle l'habileté de l'opérateur, - peut la modifier. Dans le procédé si souple du collodion lumide, des manipulations sagement conduites d'affaiblissement et de renforcement combines font apparaitre deux lignes distincles là où le développement proprement dit montrait une bande unique. Le même fait peut se produire, plus ou moins aisément, afec des couches d'autre espèce.

Ln autre caractère des plaques photographiques doit ètre indiqué. Produits artificiels, on pourrait croire qu'il est possible de domer à la couche active la sensibilité voulue aux diverses radiations, de manière que, malgré l'absence de coloration de l'image, ses parties claires correspondent, - dans la photocopie positive, - aux régions lumineuses de l'objet. Les recherches à ce sujet n'ont pas encore abouti. Le sel d'argent, ćlénent sensible, est surtout excité par les rayons très réfrangibles : bleu ot violet; les coulcurs lumineuses à l'oeil, jaune et vert, donnent du noir sur le positif. 
De là, un mançue de fidélité dans l'aspect de l'image. Les spécialités de placpues, dites orthochromatiques, panchromatiques, ont partiellement remédié à ce déliut, mais aux dépens de la clarté générale des images ; car le palliatif consiste à réduire, par une absorption convenable, l'action des rayons actiniques.

Nous n'insisterons pas sur la non-conservation des couleurs de l'objet dans l'image plontographique. On sait comment, à une éporque relativement récente, le problème a pu être résolu sans ètre généralisé. La méthode directe de H. Lippmann, application remarquable des phénomènes interférenticls dans les lames minces, le procédé de la trame coloríe interposé entre l'objectif et la couche sensible (plaques autochromes de MM. Lumière), n'ont pas encore supplanté la photographie courante : images noires et blanches. 


\section{CIIAPITRE III}

DE LA NETTETÉ DES IUAGES DANS LES INSTRLHEYTS OPTIQLES

\section{Des causes qui influent sur la nettetè} des images. - L'n objet éclairé peut, rigoureusement, être considéré comme l'ensemble de points géométriques lumineux; la même propriété convient à son image à travers un système optique. Vais la relation entre points correspondants n'est pas univoque : à un point objet s'associent tous les points d'une image contenus dans une aire plus ou moins étroite. Limage d'un point géométrique est une lache lumineuse.

S'il était indispensable qu'elle fùt un point, aucun instrument optique ne serait réalisable. I tes propriétés énoncées pour l'œil, - et aussi pour la plaque photographique, - dispensent de cette condition. Suivant le but $\grave{a}$ atteindre, il suffit que les dimensions de la tache image restent inférieures à certaines limites. Ia netteté demandée est alors alteinte, quelles que soient les dimensions dont il s'agit.

Les calculs de l'optique géométrique classique assignent au point lumineux une image ponctuelle. 
Ce résultat découle des hypothèses on approximations suivantes :

$I^{\circ}$ Le rayon physique lumineux possède les propriétés de la ligne droite géométrique; en d'autres termes, la sensation lumineuse s'annule autour de la ligne droite abstraite de propagation; la lumière ne se difffracte pas.

$2^{\prime \prime}$ L'organisation du système optique est telle que les rayons - gćométriques, suivant l'hypothèse précédente - issus d'un point, convergent vers un point à la sortie du système. Autrement dit, celui-ci ne présente pas d'aberrations dues à sa forme; il est aplanétique.

La marche des rayons est ici réglée par la loi de Descartes, l'indice de réfraction $n$ étant constant.

$3^{\circ}$ Le système optique est en outre exempt d'aberrations dues à l'inégale réfrangibilité des couleurs; il est achromatique, c'est-id-dire que les points images forniés par des rayons de colorations différentes coïncident. Sous une autre forme, à un rayon incident géométriquement simple, quoique physiquement complexe, correspond un seul rayon émergent de même nature.

L'imparfaite réalisation de ces hypothèses" transforme le point image en tache.

48. Possibilité d'une étude séparée de chaque cause. - La détermination de Ia tache aréolaire serait des plus pénibles s'il était nécessaire d'envisager l'action simul-

1 A celles-ci on doit ajouter les deux suivantes : les milieux traversés sont homogènes; les surfaces actives sont géométriquenent continues. Flles seront supposées implicitement réalisées. 
tanée des trois causes : diffraction, aberrations de forme, aberrations de réfrangibilité. Mais dans l'emploi habituel et la constitution des instruments optiques que nous considérons, les hypothèses énoncées sont suffisamment approchées pour que l'on puisse négliger les variations des causes dues à leur existence simultanée. On pourra donc étudior l'effet de chacune des causes, en supposant que les deux autres n'agrissent pas, et combiner ensuite, par simple supérposition, les trois effets résultants.

\section{Diffraction.}

49. Tache de diffraction. - Nous supposons que le système optique est aplanćlique et achromatique. Le foyer conjugué d'un point sera donc le point unique de concours des éléments rectilignes émergents des droites brisées géométriques, dont la marche à travers le système est régie par la relation analytique $\sin i=n \sin r$, droites brisées qui, d'autre part, passent par le point donné.

Tout point géométrique lumineux est un centre d'ébranlement de l'éther. Les ondes sphériques, - le milieu élant homogène, - centrées sur ce point se transforment dès leur cntrée dans le système optique; mais, celui-ci ćtant aplanétique, les ondes émergentes sont nécessairement spliériques et concaves vers le foyer conjugué du point objet. Elles créent autour de ce foycr une agitation éthérée qui se traduit par une sensation lumineuse.

La sensation serait celle d'un point si les ondes émergentes étaient physiquement illimitées; le fait ne peut se présenter, parce que les ondes incidentes sont, 
quant à leur partie utile, limitées dès leur entrée dans le système optique. L'arrêt d'une portion de l'onde par une ouverture est accompagrnée de phénomènes de diflraction qui transforment le fojer ponctuel en tache.

5o. Ouvertures ou diaphragmes apparents. - La destruction partielle de l'onde s'opére au moment où celle-ci roncontre, dans sa propagation, un diaphragme de limitation, montures des verres comprises. - La portion efficace de l'onde incidente peut être déterminće comme il suit ${ }^{1}$ :

Soit (fig. 3) D un diaphragme; formons son image $\mathbf{D}^{\prime}$ à travers la partie antérieure $\left(S^{\prime}\right)$ du système total. Si D limile réellement londetransmise, l'ouverture $D^{\prime}$ limitera virtucllement l'onde

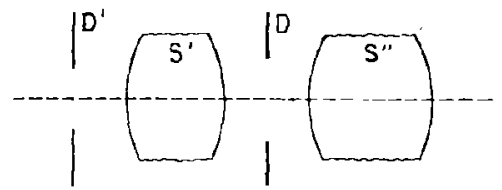

Fig. 3. incidente. En répétant la même opération, - image de chaque diaphragme ou surface de chaque verre par rapport à l'ensemble du système qui lui est antérieur, - on obtiendra des images telles que $\mathrm{D}_{1}^{\prime}, \mathrm{J}_{2}^{\prime}, \ldots$ L'onde incidente issue du point réel A sera limitée par celle des ouvertures $D^{\prime}$ qui sera vue de A sous le plus petit angle. L'ouverture on question s'appellera diaphragme apparent (ou ouverlure apparente) d'entrée.

1 D'une manière générale, les figures seront tracées de façon que la lumière se propage de la ganche vers la droite. La surface anterieure se trouvera done à gauche du lecteur. Quand cette règle ne sera pas suivie et qu'il pourra y avoir doute sur le sens de proparation de la lumière, des fleches indiqueront la marche des rayons. 
La mème règle s'applique à l'onde éntergente, les images $D^{\prime \prime}$ de $D$ étant formées par rajport à la partie postérieure du système. On obtient ainsi le diaphragme apparent de sortie.

Ia surface antérieure du système est elle-même son image; elle peut être à la fois diaphragme apparent et diaphragme réel. Ta même propriété s'applique à la dernière surface.

Il doit être entendu que la lumière est supposée se propager en sens rétrograde quand on forme l'image par rapport à un système partiel antérieur an diaphragne.

5r. Image de diffraction dans une lunette. - La théorie ondulatoire de la lumière, lascée sur le principe de Huygens-Fresnel, permet de calculer les elfets dus à la diffraction. Deux cas très fréquents conduisent à des résultats simples.

Le premier se présente dans un système convergent lorsque le point rayonnant est éloigné, à l'infini : il concerne plus spécialement la production des images réelles par les objectifs des lunettes et, dans de fréquentes circonstances, par les objectifs photographiques.

L'onde incidente est alors plane, et si le radiant ${ }^{2}$ est sur l'axc du système, l'onde est en outre normale à l'axe. L'image se présente alors. dans le plan focal principal, sous la forme d'un cercle lumineux, dit tache centrale, entouré d'anneaux alternativement sombres et clairs. Cette apparence découverts, par Herschel, étudiée par Fraunholer, expliquée, par Airy, tient aux

1 Cette expression, radiant, est due d Clairaut. 
phénomènes d'interférence qui se produisent entre les ondes élémentaires issues des divers points de la zone sphérique, en laquelle le système optique a transformé l'onde plane limitée par le diaphragne apparent.

Le rayou de la tache centrale, lorsque le système optique est plongé dans l'air, est clonné par la relation théorique suivante :

$$
p=I, 2 \frac{\lambda i}{\Omega}
$$

$F$ est ici la focale principale de l'objectif; $\Omega$, son diamètre (dit ouverture); $\lambda$, la longueur d'onde de la lumière émise.

En appelant इ l'angle, en secondes, sous lequel on voit le rayon de la tache du point nodal d'émergence (du centre optique, si l'objectif est mince), la formule peut s'écrire :

$$
\varepsilon \Omega=2 \text { 位ooo } \lambda \text {. }
$$

Cette relation, contenant $\lambda$, montro que les bords de la tache sont irisés quand le radiant émet de la lumière blanche; les anneaux clairs présentent le même caractère.

D'autre part, l'éclairement de, la tache est loin d'être uniforme; son intensité, maximum au centre, s'abaisse au tiers de sa valeur au milieu du rayon, pour devenir nulle au bord; quant aux anneaux, dont l'intensité décroît avec le numéro d'ordre, le premier ne possède qu'une intensilé maximum n'atteignant pas les o,02 de celle du centre. Aussi les anneaux sont-ils rarement discernables.

52. Pouvoir séparateur d'un objectif de lunette. - Ces considérations, vraies pour un radiant 
situé sur l'axe optique, restent suffisamment exactes pour des points voisins. Il s'ensuit que deux points angulairement très rapprochés donneront des taches identiques qui pourront empiéter l'une sur l'autre. En raison de la répartition de l'éclairement sur chacune d'elles, on peut admettre qu'elles paraîtront encore séparées lorsque la circonférence de l'une passera par le centre de l'autre. L'angle correspondant, égal à $\varepsilon$, déterminera le plus faible écartement angulaire de deux points donnant des images distinctes; il définira donc, quant à la diffraction, lo pouwoir séparateur du système optique.

En lumière blanche, on peut attribuer à $\lambda$ la valeur $0,58 \mu$ rópondant au jaune, et écrire la dernière formule :

$$
\Xi Q=\mathrm{r} 30
$$

$\varepsilon$ étant exprimé en secondes et $\Omega$ en millimètres.

Un objectif aplanétique de $\mathrm{s} 3 \mathrm{o}$ millimètres d'ouverture peut donc séparer, dédonbler la seconde. La propriété a été vérifiée expérimentalement par Foucault.

La relation précédente porte parfois son nom.

53. Pouvoir séparateur d'un objectif de microscope. - Le second des cas que nous avions en vue se rapportc à l'objectif du microscope. Ici, l'objet est à peu de distance du système optique; l'image en est éloignée, - la focale de l'objectif servant de terme de comparaison. Le rayon $;$ de la tache centrale qui répond à un point de l'axe optique est donné par la même formule que dans le cas de la lunette, $\Omega$ désignant alors l'ouverture apparente de sortie et $\mathrm{F}$ la distance à son centre du point image. La même règle peut être suivie pour la détermination 
du pouvoir séparateur. Celui-ci étant caractérisé par l'intervalle minimum $\delta$, qui, sur l'objet, sépare deux points discernables à travers l'objectif, on aura :

$$
\delta=k \frac{\lambda}{n \sin } \bar{u},
$$

$\lambda$ est la longueur d'onde, dans le vide, de la lumière émise; $n$, l'indice de réfraction du milieu interposé entre l'objet et la face, frontale de l'objectif; $u$, l'angle sous-tendu du point objet par le rayon de l'ouverture apparente d'entrée (qui cọ̈ncide généralement avec la surface antérieure de l'objectif).

La valeur du coefficient $k$ est habituellement prise ćgale à 0,5 . Elle pourrait ètre portée à 0,6 .

Ces considérations s'appliquent à l'observation d'objets lumineux par eux-mêmes ou diffusant la lumière reçue.

Quand il s'agit de l'examen d'objets à structure périodique et régulière comportant des éléments opaques et transparents (ou réfléchissants), Abbe (1) et IIelmholtz (54) ont, à peu près simultanément, et après Fraunhofer (75), indiqué, pour le pouvoir séparateur d'un objectif, la formule :

$$
\delta=k \frac{\lambda}{n \sin u} .
$$

Pour un réseau, $k$ est égal à I ou à 0,5 suivant que les rayons qui éclairent l'objet tombent sur lui parallc̀lement à l'axe de l'objectif, ou sous l'incidence maximum qui permet encore la formation d'images.

Quel que soit l'objett observé, les relations précédentes montrent que le pouvoir de résolution de l'ob- 
jectif croît quand $\lambda$ diminue, lorsque $n, u$ el par suite $n \sin u$, augmentent. On affaiblit $\lambda$ en utilisant des radiations très réfrangibles (lumière bleue; rayons violcts ou ultra-violels daus le as de la niciopliotographie); on accroil $n$ ex interposant entre l'objet et l'objectif un liquide très réfringent (ohjectif à immersion), et $u$ en ouvrant les verres et rapprochant de l'objet la surface frontale de l'objectif.

$n$ siu $u$ s'appelle, d'après Abbe, ouverlure numérique de l'objectif.

5 ł. Pouvoir séparateur do l'cil. - I'œil est un système oplique. Disposé pour la vision à grande distance, la formule de Foucault lui assure une acuité visuelle, qui est le nom de son pouvoir séparateur, égale à $33^{\prime \prime}$ pour une ouverture de pupille de 4 millimètres de diamètre. L'expérience ne juslifie pas ce nombre. La même formule ne donne l'acuité habituelle de $60^{\prime \prime}$ que pour une pupille de 2 millimètres, ce qui suppose des points lumineux intenses. Quand Ja pupille se dilate, le pouvoir séparateur reste constant, soit en raison de l'insuffisance cle clarté, soil à cause de l'apparition d'aberrations chromaliques.

55. Rôle de l'oculaire dans la séparation des images. - Grossissement. - Qu'il s'agisse d'images réelles données par ur objectif de télescope, de microscope, ou par un photo-objectif, l'intcrvalle linćaire entre deux taches séparces est trop faible pour être discernable à la vision directe. Il faudrail on effet que l'oil pút se placer de telle sorte que l'angle déterminé par les droiles joírnant an noud antécieur les centres des deux taches, fùt au moins égal à une minute. Généralement, cette condition amèncrait l'image 
en deçà du punctum proximum, là où l'accommodation est impossiblè.

On sait qu'on tourne la difficulté en observant l'inage à havers un système optique, l'oculaire, qui, en la rendant virtuelle, rejette l'image au delà du puntum proximum. L'écart angulaire des deux détails, des deux points séparés, prend alors une valeur déterminée; son rapport à l'écart des points correspondants de l'objet, - celui-ci étant supposé vu directement par l'oeil, dans les meilleures conditions d'observation possibles, - n'est autre que le grossissement de l'instrument (ensemble des systèmes oljectif et oculaire).

Le système objectif étant supposé donné, ainsi que les deux points juste séparés par lui, l'angle visuel à travers l'oculaire est essentiollement fonction de la focale de ce dernier et de sa position, qui détermine la situation de l'image virtuelle. Le grossissement ne dépend plus que de cet angle visuel, et varic proportionnellement à lui.

L'angle visuel doit être au moins égal à une minute. Cette condition détermine la valeur du grossissement minimum de l'instrument. Il n'y aurait pas lieu d'envisager un grossissement masimum, si toute augmentation de cette qualité secondaire n'était accompagnée pour Ies autres qualités essentielles (le champ, la clarté et quelquefois la fidélité des images) d'une diminution qu'on ne peut annuler sans changer le système objectif. Le minimum dı grossissement peut donc être aussi considéré comme sa valeur maximun, et par suite comme sa valeur normale'.

1 Les personnes dont l'acuilé visuclle est diflérente de la minute doivent modifier, en conséquence, le grossissement. 
Il faut toutefois corriger la précision de cette conclusion par la remarque suivante : "Certaines personnes distinguent quelquefois mieux dans mne image légèrement confuse et avec un angle visuel plus grand qu'avec une accommodation exacte et un angle de vision moindre (53). "

Remarquons encore qu'une augmentation de grossissement peut, d'elle-même, contribuer à la netteté : deux taches qui se recouvrent plus qu'il ne faut pour être séparées peuvent être distinguées gräce à un fort grossissement; il suffit que la diminution de clarté soit assez grande pour rendre insensible à l'oeil la région médiane et assez faible pour conserver aux deux centres une intensité convenable.

56. Grossissement normal des lunettes et des microscopes. - Les considérations précédentes seront appliquées aux lunettes et au microscope.

Dans les lunettes, il est préférable que l'image virtuelle soit rejetée à l'infini : l'ceil observe alors an repos avec le minimum de fatigue. Le grossissement de l'instrument est égal au rapport des focales de l'objectif et de l'oculaire :

$$
\mathrm{G}=\frac{\mathrm{F}}{f} .
$$

Pour un pouvoir séparateur $\varepsilon$ secondes, l'angle visuel, ayant pour valetr $\mathrm{G} \varepsilon$, devant être égal à $6 \mathrm{o}^{\prime \prime}$, on aura :

$$
G=60 .
$$

La relation de Foucaull et les équations précédentes donnent : 
$\mathrm{G}=\frac{6 \mathrm{o}}{\varepsilon}, \quad \mathrm{G}=\frac{60}{130} \Omega=0,462 \Omega ; \quad f=2,16 \frac{\mathrm{F}}{\Omega}$, ( $\Xi$ en secondes, $\Omega$ en millimètres').

Le grossissement normal est donc un peu inférieur au rayon, exprimé en millimètres, de l'ouvorture utile de l'objectif'.

Pour le microscope, l'image est amenée à la distance $\Delta$ du punctum proximum. I'observation se fait alors dans des conditions les plus favorables. Si l'intervalle $z$ paraît sous l'angle $\alpha$, on aura, G étant le grossissement linćaire :

$$
G=\frac{\Delta x}{\delta} .
$$

En faisant $\alpha=\mathbf{I}$ : 3000 (valeur de la minute) et $\Delta=250$ millimètres, on obtient pour le grossissement normal :

$$
\mathrm{G}=83 \frac{n \sin u}{\mathrm{~K} \lambda}
$$

$\lambda$ étant exprimé en microns.

$\mathrm{Si} \mathbf{F}, f$ sont les focales respectives, dens l'air, de l'objectif' et de l'oculaire, l'optique géomélrique donne la relation :

$$
\mathrm{G}=\frac{n \mathrm{~J}^{2}}{\mathrm{~F} f},
$$

qui détermine l'oculaire à associer à un objectif de constantes connues.

57 . - Quelles que soient les causes, diffraction ou aberrations, qui, au lieu de points, produisent des taches images, les considérations précédentes s'appliquent à la détermination du grossissement normal. Si la question a été exposée ici, c'est que la diffraction doit la régler. 
En effet, les éléments d'un système optique doivent c̀tre déterminés de façon que les taches dues aux aberrations soient au plus égales à la tache diffractive. Lorsque la condition ne peut être réalisée, il y a lieu de diminuer l'ouverture de l'objectif : cette modification facilite la réduction des aberrations et augrmente la tache due à la diffraction. C'ost done, en définitive, ce dernier phénomène qu'il reste à considérer dans le sujet actuel.

\section{Aberrations de sphéricité; aplanétisme.}

58. Image solide. - L'hypothèse fondamentale est, ici, l'assimilation du rayon lumineux à la droite géométrique; elle supprime tout effet de diffraction. I.e système optique est, en oulre, supposé achromatique; sous une autre forme, on ne considère que des rayons homogènes, non décomposables, monochromaliques.

Les rayons issus d'un point lumineux, rayons homocentriques, ne possèdent pas, à leur émergence d'un système optique, la propriété de passer par un point unique, soit réel si le rayon Iumineux le rencontre, soit virtuel s'il en est ainsi de son prolongement géométrique.

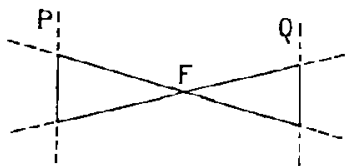

Fig. 4.

Si le caractère d'homocentricité était conservé, le point de concours F (fig. 1), foyer du point objet, aurait le rôle suivant: c'est par lui que devrait passer le plan d'une plaque photographique de finesse idéale; c'est sur lui que devrait s'accommoder un wil parfait, pour perce- 
voir avec la netteté maximum les détails de l'objet dont le point radiant fait partie.

La perfection n'existant pas, il sera cependant tout aussi favorable, pour la perception de la netteté, que l'wil s'accommode entre deux plans, P el Q, tels que les sections par eux du faisceau émergent soient vues de l'oeil sous un angle au plus égal à une minute; la plaque sensible pourra ètre disposée entre les deux plans analogues qui détermineraient dans le faisceau des sections égales au cercle de diffusion lolérée. Quel que soit l'organe récepteur, wil ou plaque, nous appellerons plan de mise an point le plan sur lequel la plaque est située ou l'œil accommodé.

Les rayons émergents ne restant pas homocentriques, le faisceau, au lieu d'être un còne à double nappe, deviendra (fig. j) un quasicône, surface régrlée à cercle de gorge $\Phi$ ou non ponctuel. Si le diamètre de $\Phi$ est assez faible, il existera, comme dans le cas précédent, deux plans limites $P$ et $Q$ de mise

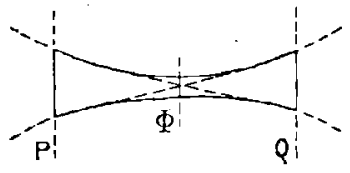

Fis. 5 . au point. Ia netteté des inages restera aussi parfaite que dans un système aplanétique; mais la profondeur de mise au point, intervalle des plans $P$ et $Q$, sera vraisemblablement plus faible. Ce défaut pourra se traduire par une fatigue de l'ceil résullant d'une tension de l'accommodation entre des limites très étroites, et par une difficulté de mise au point de la plaque tenant à la précision exigée. I.e solide quasi-conique, limité par les plans $P$ et $Q$, constituera, comme pour l'œil, ce que nous appelons une-image solide.

Instruments optigaues. 
59. Théorème de Dupin. - En donnant au rayon lumineux les propriétés de la droite, on réduit, gràce à la loi de Descartes, toule l'étude des aberrations à une question de gćométrie pure. La thćoric est dominée par un théorème remarquable, auquel se rat tachent les noms ${ }^{1}$ de Walus. de Dupin et de Sturm, du second surtout.

Cette proposition, susceptible d'un énoncé purement géonétrique très général, peut, relativement à notre sujet, être ainsi formulée : Les rayons issus d'un point lumineux, et traversant un système optique, sont, après chaque réflexion ou réfraction soumise à la loi de Descartes, normaux à une même surface géométrique (de forme variable après chaque réfraction); par suite, les rayons infiniment voisins d'un rayon quelconque donné, dit médial, s'appuient sur deux éléments linéaires per pendiculaires entre eux et au rayon médial.

La surface trajectoire normale des rayons refractés, nous l'appellerons surfare de Dupin. Les éléments linéaires d'appui sont les droiles focales de sturm; elles passent par les centres de courbure de la surface de Dupin situés sur le rayon médial, et appartiennent à ses plans principaux.

La surface de Dupin n'est d'ailleurs autre chose que la surface de l'onde; nous lui donnons un nom particulier pour rappeler son origine purcment géométrique.

\section{6o. Aberration d'un faisceau issu d'un point} de l'axe. - La présence d'un axe dans les systèmes centrés conduit tout naturellement ì considérer d'abord

1 On attribue souvent ce théorème d̀ Gergonne. C'est faire trop dhonneur à ce dernier. (Ann. de Math., XIV, 1823-21.)

IRIS - LILLIAD - Université Lille 1 
le cas où le radiant P cst sur cet axe (fig. 6). I Les surfaces de Dupin, la surface d'émergence $\Sigma$, en particulier, sont de révolution autour de l'axe. Ie pinceau ${ }^{t}$ central émerge suivant un pinceau conique dont. le sommet est au centre de courbure unique $\mathrm{P}^{\prime}$ de $\Sigma$ sur l'axe PX. Un pinceau marginal de direction PI émerge suivant un pinceau dont le rayon nuédial est EQ ot

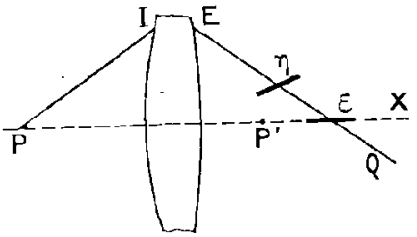

Fig. 6. qui s'appuie sur les deux droites focales $\varepsilon$ et $r_{1}$ - Le plan II, commun à l'axe et aux deux rayons médiaux, est pris comme plan du tableau. - La focale $\varepsilon$ est située sur l'axe $P \lambda$, car clle ne doit évidemment pas se déplacer quand PI tourne autour de PX; l'autre focale, $n$, est par suite normale en $h$ à LQ et au plan II. Lorsque le pinceau PI tourne autour de PX pour engendrer une couronne incidente, $\varepsilon$ reste fixe, et $r_{i}$ enveloppe une circonférence de cercle $\gamma$, lieu du point $h$, normale à l'axe et centrée sur lui. Quand, enfin, l'iuclinaison de PI sur l'axe varie de manière à faire balayer à la couronue incidente la surface utile d'entrée, l'élément $\varepsilon$ décrit une portion finie de l'axe, et la circonférence $\gamma$ engendre une zone, limitée par deux plans normaux à $\mathrm{PX}$, de la surface caustique $\mathrm{T}$ d'émergence.

En résumé, les rayons émergents formeront un complexe s'appuyant (fig. 7 ) sur le segment $P_{1} P_{2}$

1 Nous réservons le nom de pinceall à un faisceau infiniment delic. 
de l'axe et sur une zone de surface I' limitée par les plans $\mathrm{MI}_{1}$ et $\mathrm{M}_{2}$. La méridienne de cette surface de révohution passera d'ailleurs par le foyer $F^{\prime}$, où l'axe sera une tangente dè rebroussement. La section déterminée dans ce complexe par un plan de mise au point constituera l'image du point $\mathrm{P}$ sur ce plan.

Cette image aura l'aspect général suivant : un point central très brillant entouré

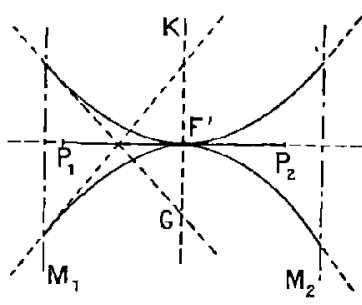

Fig. 7. d'un cercle plus sombre; une circonférence lumineuse, section de la surface canstique, bordera ce cercle et sera suivie d'une couronne circulaire dégradéc.

Cette couronne pourra fitire défaut si ce jlan de mise au point est asse\% éloigné de $\mathrm{F}^{\prime}$; le point central sera moins accusé en dehors du segrment $P_{1} P_{2}$. Nais, de tous ces plans de mise au point. le plus remarquable est celui $\mathrm{K}$ qui passe par $\mathrm{F}^{\prime}$ : la circonférence lumincuse se réunit au point central augucl elle donne l'intensitć maximum. Cette propriété fait attribuer à la tache correspondante la qualité d'être la meilleure image, bien que son diametre ne soit pas minimum; ce demier caractìre appartient à la section détcrninée par le plan $\mathrm{N}_{1}$ ou $\mathrm{N}_{2}{ }^{(1)}$.

Dans un grand nombre de systèmes usuels, $\mathrm{P}_{2}\left(\right.$ ou $\left.\mathrm{P}_{1}\right)$ coïncide arec $\mathrm{F}^{\prime}$. Le rayon $\mathrm{F}^{\prime} \mathrm{G}$ de la tache sur le plan $\mathrm{K}$ s'appelle l'aberration latérale; le segment $\mathrm{F}^{\prime} \mathrm{P}_{1}\left(\right.$ ou $\mathrm{F}^{\prime} \mathrm{P}_{2}$ ) est l'aberration longitudinale. On prut clonner un signe

(1) Le lecteur verra aisément la position des plans $N_{1}$ et $N_{2}$ Eur la figure 7 . 
à cette dernière, suivant la posilion relative des points extrèmes.

61. Séparation des taches de diffusion. Un radiant voisin de l'axe donne, par raison de continuité physique, une image sensiblement identique à celle d'un point axial. Les taches de deux points roisins angulairement (el linéairement, s'ils sont à distance finie) pourront empiéter l'une sur l'autre. On admet, pour leur séparation, soit la règle nême qui régit les taches diffractées, soit plutót celle qui assure la langence extéricure des taches.

62. Aberration d'un pinceau oblique; astigmatisme. - Nous supposons maintenant le radiant en dehors de l'axe du système optique. Le pinceau PI (fig. 8) donne un jinceau émergent s'appuyant sur les deux droites focales $\varepsilon$ et $r_{i}$, dont la situation exacte dans lc systime ne nous est pasindispensable. Si

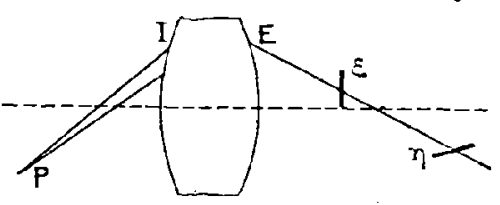

Fig. 8 . le pinceau s'ouvre de manière à se transformer en un faisceau, de rayon médial PI, découpant sur la surface incidente une aire petite, mais finie, le théorème de Sturm s'applique encore dans son essence. Les éléments $\varepsilon$ et $\gamma$ deviennent des droites physiques, c'est-a-dire présentant une petite épaissenr, et courtes, mais non infiniment petites; toutes les normales autour de $\mathrm{E}$ ne compent pas ces droites physiques (car il n'y a plus ici de termes infinitésimaux du troisième ordre, mais seulement des quantilés très faibles). Quelques-unas 
d'entre elles y passent seulement fort près; de sorte qu'en mettant au point sur $\varepsilon$, par exemple, l'imare de $P$ sera une petite droite lumineuse entourée d'une aire de diffusion allongée dans sa direction. En $r_{i}$, l'image - aura le même caractère, mais sa direction sera à $90^{\circ}$ de la première. Dans l'intervalle, l'inage se rapprochera de la forme circulaire, qu'elle atteindra en un point déterniné; mais l'intensité centrale sera plus faible que celle des images linćaires, et la dégradation lumineuse moins rapide. Fin dehors de l'intervalle $e r_{i}$, l'image aura une forme elliptique dont la grandeur absolue ira constamment en augrmentant.

Supposons, pour abréger le discours, le système orienté de façon que $\varepsilon$ soit vertical. Imaginons, au lieu de $P$, une petite croix dont une branche soit verticale. Ia mise au point sur $\approx$ donnera une image (fig. 9)

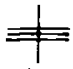

(a)

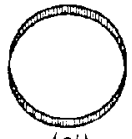

(a')

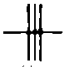

(b)

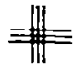

(c)

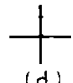

(d)

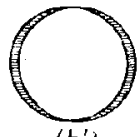

(b')

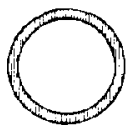

(c')

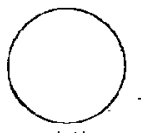

(d')

Fig. 9.

ayant l'apparence $(a)$; la mise au point sur $n$ donnera l'aspect $(b)$; la figure (c) conviendra à une mise au point intermédiaire.

Si la croix est remplacée par une circonférence de cercle d'égale ćpaisseur, les apparences seront respecti- 
vement celles $\left(a^{\prime}, b^{\prime}, c^{\prime}\right)$ figurées ci-dessus. Nais, dans aucun cas, l'image n'aura l'aspect $(d)$ ou $\left(d^{\prime}\right)$, qu'on obtiendrait en centrant la figure sur l'axe optique de l'instrument.

Les apparences dont il vient d'être question portent le nom général d'astigmatisme ${ }^{1}$; il n'y a pas, en effet, alors, de foyer conjugué; en place, deux droites lumineuses espacées et un intervalle dans lequel on a le choix des images possibles. Les deux focales de Sturm, physiquement réalisées, sont les droites diastigmatisme. Leur intervalle s'appelle, parfois, l'astigmation.

63. Aberration d'un faisceau oblique; coma. - Envisageons, maintenant, le cas où le faisceau incident intéresse toute l'ouverture d'un système optique centré. Le pinceau $\mathrm{PN}^{\prime}$ (fig. ro), dirigé vers le centre $\mathrm{N}^{\prime}$ de l'ouverture apparente d'en trée, émerge suivant un

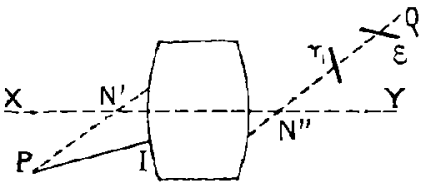

Fig. 10. pinceau tel que $N^{\prime \prime} Q$ passant par $N^{\prime \prime}$, centre du diaphragme de sortie.

Les focales du pinceau sont l'une $\approx$ normale au plan $(P, x y)$, l'autre $\eta_{\text {dans }}$ ce plan; des considérations de symétrie suffisent pour l'établir.

Quand le pinceau incident PY' se déplace et, tel PI, balaye l'ouverture d'entrée du système optique, de façon à engendrer le faisceau total, les focales $\varepsilon$ et $\eta_{\text {r varient. }}$ Elles ne peuvent envelopper des cercles qui devraient être à la fois centrés sur $x y$ et sur $\mathrm{PN}^{\prime}$; elles restent

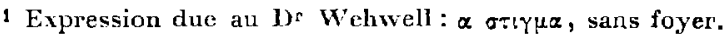


tangentes à deux sortes d'ellipses, dont les grands axes sont respectivement parallèles aux directions indiquées $\varepsilon$ et $r$ des focales du pinceau $Q^{\prime \prime}{ }^{\prime \prime}$. Ces ellipses, d'autant plus aplaties que l'ouverture est faible le faisceau peu ouvert et peu incliné sur l'axe, constituent les meilleures - ou, plus exactement, les moins mauvaises images du point $\mathbf{P}$, parce qu'elles possèdent l'intensité spécifiçue maximum qui attire l'seil et impressionne fortement la plaque sensible. Leur ensemble se substitue au foyer conjugué $\mathbf{F}^{\prime}$ du point situé sur l'axe, point commun à l'élément et à la caustique, lieu et enveloppe respectifs des focales de Sturm.

Pour les neêmes causes qui interviennent lorsqu'il s'agit d'une image axiale, les ellipses lumineuses, squeletles de l'image astigrmatique, sont accompagnées d'une aire de diffusion de même forme. Au point de vue de l'intensité lumineuse, cette aire, symótrique par rapport au plan Pxy du radiant et de l'axe optique, ne l'est pas relativement au plan normal à $\mathrm{P} x y$ et pissant par $\mathrm{QN}$ ". En effet, l'image astigmatique due au faisceau total est la résultante par superposition des images analogues formées par les faisceaux déliés tels que PI, images élémentaires qui sont sensiblement symétriques autour du rayon émergent médial. Considérons une grerbe conique de ces faisceaux $P^{\prime} l$ de révolution autour de $\mathrm{PY}^{\prime}$; l'image qu'elle produit reste à peu pres symétrique autour de QN" tant que la base de la couronme s'appuie tout entière sur la surface utile d'entrée du système optique; en ouvrant de plus en plus la gerbe de façon a lui faire balayer toute celte surface utile, il arrive, en raison de la non-symétrie du système autour de $\mathrm{QN}^{\prime \prime}$, qu'une partie, de plus en plus faible, de la couronne 
atteint la surface utile. Ces régions provoquent sur l’image déjà formée une traînée luminense dont l'axe est, dans le plan P $x y$, normal à $Q X^{\prime \prime}$ et qui s'affaiblit en s'éloignant de cette dernic̀re droile. La traînée forme ainsi la quene ou chevelure d'une sorte de comète qui devient l'image astigmatique; c'est cette apparence que les auteurs allemands désignent sous le nom de coma.

64. Travail des surfaces dans les systèmes optiques. - Vous venons de considr'rer trois cas de production d'aberrations : faisceau émanant d'un point axial; faisceau issu d'un point quelconque avec deux variantes, suivant que la base du faisceau n'inléresse qu'une pelite région d'une surface réfringente ou conve totalement celle-ri.

Le premier cas concerne évidemment l'observation centrale par les instruments, et s'applirque alors à toutes les surfaces des systimes. Il faut, pour les denx autres, distinguer le caractère de ces surfaces au point de vue de leur mode de travail. Les unes travaillent sur toute leur ouverture efficace pour le faisceau utilisé émanant d'un radiant donné (ce sont, par exemple, les objectif's des lunettes astronomiques et des microscopes, les oculaires des lunettes de Galilée); les autres ne travaillent, pour le nême pinceau, que dans une petite région qui se déplace on mêne temps que le radiant (telles les surfaces des oculaires astronomiques et terrestres, de l'objectif de la Iunette de Galilée). Dans les objectifs photographiques doubles, les surfaces travaillent totalement ou presque, surtout pour les appareils ramassés; - le travail est partiel pour les objectifs simples diaphragmés à distance, et les objectifs triples quant aux surfaces des combinaisons postérieures. 


\section{Correction des aberrations. Formule de}

Goulier. - La correction des aberrations est un problème des plus délicats. Yous ne donnerons ici que des indicalions d'ordie général.

Dans le cas de l'observation axiale, le but poursuivi doit être de rédnire à la valeur maximum adnissible l'aberration latérale, c'est-à-dire de réaliser l'aplanótisme suivant l'ave.

Pour les objectifs photographiques, on a d'abord le choix entre deux palliatif's : diaphragmer ou employer des objectifs a long foycr; car, grosso modo, la valcur de l'aberration latérale est de la forme $\mathrm{II} \frac{u^{3}}{f^{2}}, u$ étant l'ouverture et $f$ la focale principale de l'appareil. Mais le choix de la focale est souvent imposé par des conditions d'emploi, et la fermeture du diaphragme est limitée par les effels de diffraction. La véritable réduction doit porter sur le facteur M qui met en jeu les positions relatives des surfices des verres. Relativement aux instruments comportant objectif et oculaire, la question peut être scindée, chaque combinaison étant étudiée à part.

Pour les lunettes, le colonel Goulier (39) a énoncé la règle suivante : "On peut démontrer, par le calcul, que dans les lunettes de méme qualité, c'est-à-dire exécutées avec la mème perfection et ayant d'ailleurs la mème clauté, les cercles de confusion ont le mème rayon, ou, autrement dit, le défaut de netteté apparente des objets visés est le même quand le rapport de la longueur focale $f$ de l'objectif au diamètre $\Omega$ de son ouverture efficace est proportionnel à la racine quatrième de la longueur focale. Si on détermine le 
rapport de proportionnalifé en admettant que pour $f=3$ oo millimètres, $\Omega=30$ millimètres, on trouve la formule suivante, dans laquelle $f$ et $\Omega$ doivent etre exprimés en millimètres :

$$
f=\frac{\sqrt[4]{f}}{0,4 \lambda} .
$$

... Toutefois, la formule ci-dessus suppose qu'on a adopté, pour les quatre surfaces des lentilles, les rayons de courbure les plus avantageur, et que les lentilles sont bien centrées."

La relation de Goulier peut encore s'écrire :

$$
\Omega=0,42 \sqrt[4]{f^{3}} \quad \text { ou } \quad f=0,318 \sqrt[3]{\Omega^{4}}
$$

La forme en était connue au xvme siècle $(\mathbf{8 1})$; le coefficient seul paraît avoir été déterminé par Goulier. La formule aneienne concernait uuc lunet te composée d'un objectif et d'un oculaire, lous deux simples. Yous ne pensons pas qu'on puisse la justifier, et, comme on l'a fait quelquefois, l'appliquer sans discussion pour la détermination des focales d'objectifs à deux verres d'ouvertures données. Goulier corrigre d'aillenr's l'absolu de la formule en disant : "On admet que, pour les lunettes d'instruments bien exécutées, le rapport de l'ouverture à la longueur focale de l'objectif pent varier en général entre $\frac{\mathrm{I}}{\mathrm{I}} \mathrm{z}$ et $\frac{\mathrm{I}}{\mathrm{I} O}$, ef aller exceptionnellement a $\frac{\text { I }}{9}$. " I a compétence de l'auteur est la garantie de celte remarque, au moins pour les elppareils organisés, comme les longues-vues de modèle courant.

Dans les luneltes de Galilée, toujours peu puissantes 
et d'olganisation simple, ]'aplanétisme général, aussi bien central qu'oblique (nous ne reviendrons pas sur co dernier), - est suffisamment obtenu en donnant a l'ouverture totale de l'objectif une valeur qui est en général le 5 ; et atleint an plus le $\begin{array}{r}\text { I } \\ 5\end{array}$ cle sa focale. Ia relation de Goulier, supposée exacte, ne devrnit pas être, ici, appliquée. à l'ouverture totalo $\Omega$, mais seulement à l'ouverture utile $\Omega^{\prime}$; celle-ci est, pour un radiant et son imagre situés à l’infini, égale à Ga environ, G étant le grossissement et a l'ouverture de la pupille. Les équations :

$$
\Omega=\frac{1}{4} \mathrm{~F}, \quad \Omega=\mathrm{G} a, \quad \Omega^{\prime}=0,12 \mathrm{~V}^{\mathrm{a}}
$$

conduiraient alors à l'expression :

$$
\boldsymbol{C}=\mathrm{I}, \mathrm{I} 9 \underset{a}{\mathrm{I}} \sqrt[4]{\mathrm{Q}^{3}}
$$

indiquant le grossissement normal d'une lunette galiléique pour des ouvertures totales de l'objectif et de la pupille données. La pratique justifie assez bien cette conclusion.

Enfin, dans les objectifs de microscopes, la correction des aberrations est facilitée par l'existence de points aplanéliques dont la découverte appartient au botaniste Lister (6). Pour des radiants qui en occupent la place, les séries entières qui expriment les aberrations latérales des images ne commencent qu'au terme en $u^{\phi}$, $u$ étant l'ouverture du pinceau central. L'absence du terme en $u^{2}$ assure a l'aberration une valeur de minimum pratique.

66. - Dans les oculaires de types anciens, consti- 
tués par une même espèce de verre, l'aplanétisme a été obtenu, à la suite d'essais pratiques basés, peutêtre, sur des considérations théoriques générales, par une disposition et des formes convenables des éléments constituants. Les procédés de calcul des objectifs photographiques ont dû être employés dans l'étude des oculaires plus récents à lentilles composées.

67 . Correction de l'astigmatisme. - Lorsque le faisceau incident émane d'un point situé en dehors de l'axe optique, la correction des aberrations doit consister d'abord dans la réduction de la différence astigmatique, puis dans l'atténuation du coma et la diminution de la zone diffusée.

C'est dans les abjectifs photographiques que le problème se présente avec sa complexité maximum, et cela à cause de leur grande ouverture et de la forte inclinaison des faisceaux sur l'axe. Pour ceux qui sont très ouverts, ce n'est qu'à la suite de calculs pénibles et d'essais con̂teux qu'on peut assurer l'aplanétisme général du champ.

Quand les prévisions ne sont pas réalisées, le jeu du diaphragme peut pallier les défauts de l'organe, mais au détriment dè la clarté, jusqu'à I'apparition des taches de diffraclion : la réduction de l'ouverture, sans changer l'astigmation, rétrécit la zone de diffusion et affaiblit le coma.

Pour les objectifs de lunettes, le défaut asstigmatique est généralement peu accentué, souvent négligeable, à moins que le champ ne soit considérable. Dans ce dernier cas, le problème précédent se pose de nouveau; sinon, la correction de l'astigmatisne est une conséquence de l'atténuation de l'aberration centrale.

Instıuments optiụues. 
Ce sont surtout les ocnlaires qui peuvent donner rimage astigmatique. Il n'y a, dans ce cas, que des faisceaux peu ouverts qui agissent; le coma est négligeable; toute la question se résume dans la réduction de l'astigmation. Le guide dans la recherche de la solution paraît être dans ce fait qu'un faisceau normal à une surface réfringente n'est pas astigmatique. Il est, par suite, vraisemblable que le défaut astigmatique s'atténuera si, pour chaque lentille, le faisceau tombe, à peu près normalement, sur sa surface moyenne. On pourra y arriver autant par la forme appropriée des faces du verre que par la position de la lentille relativement au diaphragme limitatif du faisceau. L'exemple le plus typique sc rapporte, non à un oculaire, organe trop complexe, mais à l'objectif photographique simple : un aplanétisme général suffisant est obtenu à l'aide d'un ménisque concave vers l'objet el d'un diaphragme situé en avant de la lentille. Le diaphragme ne réduit aucun des intervalles des focales de Sturm; son rôle est uniquement de sélectionner les faisceaux incidents de manière à ne laisser tomber sur la surface que ceux auxquels cette dernière assure une homocentricité acceptable.

68. Aberrations sur-corrigées et sous-corrigées. - En étudiant séparément les aberrations des combinaisons - objectif et oculaire, par exemple d'un système optique et de lcurs ćléments individuels, on peut arriver à discerner les formes générales les plus convenables à leur donner; la méthode est encore commode dans un exposé didactique. Il serait, cependant, désavantageux de l'utiliser dans ses lointaines conséquences. 
Le problème, en effet, est uniquement de donner à l'image défnitive une netteté déterminée. Il n'est pas nécessaire que les images intermiédiaires possèdent le même caraclère. En s'occupant à le leur donner, on restreint gratuitement les limites entre lesquelles on peut faire varier la forme ou la situation des verres constituants, pQur satisfaire à la propriété demandée; on complique done la question.

Si on se borne à réaliser la netteté exigée, seulement dans l'image définitive, les images antérieures pourront ètre plus nettes on moins nettes que si elles possćdaient la même sćparation des détails que l'image dernière. Dans l'image antérieure en question, l'aberration sera dite, suivant le cas, sur-corrigée ou sous-corrigée.

\section{Aberrations de rëfrangibilité. Achromatisme.}

69. Images solides chromatiques. - Suivant la remarque antérieurement faite, les systèmes optiques que nous aurons à considérer seront supposés aplanétiques. Nous raisonnerons d'ailleurs, pour fixer les idées, sur un objet lumineux situé dans un plan normal à l'axe du système.

Un objet émettant des rayons non-monochromatiques, soit directement en temps que source, soit par suite de la nature de son éclairement, donne, à travers un système optique, autant d'images que la lumière incidente comporte de radiations. Les images seront situées dans des plans parallèles qui ne couperont pas l'axe au même point, et leur grandeur absolue variera 
d'un plan à l'autre; en outre, elles pourront être incomplètes; car, par exemple, si une région de l'objet (point, détail ou aire étendue) n'envoie que de la lumière verte, l'image de cette région manquera dans tous les plans autres que celui qui répond à la radiation verte.

Si l'on pouvait ne percevoir qu'une seule de ces images, le seul défaut à relever serait I'infidélité de la représentation; mais l'ćlimination nécessairc à cet effet étant souvent irréalisable, la perception à travers le système s'accompagne en outre d'un défaut de netteté.

Un point émettant des radiations diverses, au nombre de deux, R, V, pour abréger le discours, donnera deux images solides de couleurs différentes et

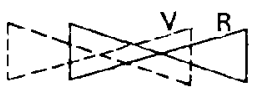

Fig. 11. non superposées (fig. I I). En supposant la mise au point (par l'ceil ou la plaque) effectuéc au centre de l'une R, l'innage reçue compor-' tera une couronne $\mathrm{V}$; et le diamètre de celle-ci pourra, suivant la distance des centres $r, v$, être supéricur au cercle de diffusion lolérée; en d'autres termes, les taches images provenant de deux points objets voisins pourraient se recouvrir plus que ne le comporte la netteté prévue pour l'instrument.

Laissons d'ailleurs de côté la modification apportée à la couleur $R$ du point central par la tache $V$, et aussi les apparences de limage quand la mise au point varie; mais signalons que, même si la tache totale est suffisamment restreinte, son aspect - cas de la lumière incidente blanche - produit à l'œil une impression désagréable, qui peut avoir une répercussion sur la netteté de perception. 


\section{Achromatismes absolu et apparent. -} En thèse générale, la réalisation de l'achromatisme exige la superposition, point par point, des images colorées ultimes du système optique; elle nécessite donc d'abord la coïncidence des foyers conjugués de l'objet, qui amène celle des plans des images; ensuite l'égalité du grossissement de ces images, ce qui conduit à la superposition de leurs bords. Suivant la méthode et les expressions de Biot, on peut rattacher à ces deux conditions deux općrations concernant respectivement l'achromatisme longitudinal et l'achromatisne latéral.

$A$ ce mode d'achromatisme, nous adjoindrons le qualificatif d'absolu. Euler (24, lettre 87) a le premier fait remarquer que cette sorte d'achromatisation, évidemment suffisante, n'était pas toujours nécessaire : quand " les dernières images représentćes par la lunette sont tellement arrangćes que la droite terminatrice, étant prolongée, passe précisément dans l'oil, alors l'œil verra 'par un seul rayon toutes les extrémités des images; et en général tous les points qui répondent̀ à un même point de l'objet seront portés dans l'œil par un mème rayon, et par conséquent ils y seront représentés distinctement ». Sous une autre forme, il suffira, pour que l'achromatisme soit réalisé, que les images colorées soient des perspectives de l'une d'elles, le point de vue étant unique; en plaçant l'œil à celui-ci, les images se projetant l'une sur l'autre donneront la sensation d'une image unique conservant les couleurs de l'objet; car, dit Biot, "l'oill tolère assez aisément les distances différentes des imaros, mais non leur défaut de direction (8). "

Ce genre d'achromatisme sera qualifié d'appa- 
rent ${ }^{1}$. Biot (8) donnait à la condition qui le concerne le nom de dispersion rectiligne des foyers.

71. Achromatisme absolu. - Choix des radiations. - L'achronatisme absolu est celui des objectifs photographiques, car la plaque sensible ne peut être traitée comme l'œil; il doit aussi être celui des objectifs de microscope, afin de rendre possible leur échange ainsi que celui des oculaires.

Remarquons d'abord qu'à priori on doit douter qu'il soit rigoureusement réalisable si, - - tel le cas de la lumière solaire, - la lumière incidente comprend une infinité de radiations formant un ensemble continu; car on serait conduit à réduire en un plan unique tout l'espace compris entre les plans des images extrêmes. La pratique se contente de solutions approximatives; il suffit que l'achomatisme soit accompli pour deux ou trois radiations, quatre très exceptionnellement.

C'est de leur choix qu'il faut d'abord s'inquiéter; on les définit par les raies correspondantes du spectre solaire.

Quand il s'agit de systèmes ou d'éléments d'instruments pour lesquels l'weil entre seul en jeu, - objectifs de lunettes dans lesquels on lient à réaliser plus ou moins parfaitement l'achromatisme absolu, objectifs de faibles numéros pour microscopes, - il suffit d'assurer la coüncidence dos images répondant aux radiations $\mathrm{D}$ et $\mathrm{F}$, les plus agréables à l'oeil : $\mathrm{D}\left(589,5 \mathrm{p}_{\mathrm{\mu}}\right)$, raie jaune du sodium; $F(486, x \mu \mu)$, raie verte de l'hydrogène.

Pour les objectifs photographiques d'usage courant.

1 Il est pratiquement réalisé dans la loupe simple. Goulier (39) lui donne le qualificatif de latéral.

IRIS - LILLIAD - Université Lille 1 
le choix de deux radiations très réfrangibles, dans le bleu et le violet, serait acceptable; mais, alors, la mise au point sur l'inage optique (jaune ou verte), la plus aisément perceptible à l'œil, laisserait en avant l'image actinique efficace pour la plaque sensible. En substituant alors cette dernière à la glace dépolie, l'image photographique serait trouble, à moins qu'on ne prît la précaution de déplacer d'une quantité convenable en avant le chàssis de la chambre. On s'évite cette sujétion en assurant, dans la mesure du possible, la coüncidence des images optique et actinique, c'est-àdire en réalisant l'achromatisme pour deux radiations assez éloignées : l'une est définie par la raie D; l'autre par la raie $G^{\prime}(434, x$ u. $)$ indigo de l'hydrogène, ou H( $(396,9)$ violette du cadmium.

Dès qu'on a affaire à des objectifs plus délicats, objectifs de microscopes de grande puissance susceptibles d'être employés avec la lumière violette, photoobjectifs utilisés soit pour la production d'images destinées à la reproduction trichrome, soit avec des plaques panchromatiques, - on peut être amené à assurer la coincidence de trois radiations assex distantes du spectre, telles que $\mathrm{C}\left(656,3_{2} \mathrm{i}_{\mathrm{i}}\right)$, raie $\alpha$ de l'hydrogène, $\mathrm{F}$ et $\mathrm{G}$ '; avec remplacement possible, suivant les cas, de $C$ par D, ou de $\mathrm{G}^{\prime}$ par la raie violette de mercure.

\section{Achromatisation de deux couleurs. -} La seule coïncidence des foyers répondants à deux ou trois radiations, effectuée sans précautions, ne suffit pas toujours à assurer un achromatisme absolu, pas même un achromatisme longitudinal convenable.

Prenons, pour fixer les idćes, le cas de la lumière blanche, et supposons qu'on ait réalisé l'achromatisme 
pour deux couleurs $\mathbf{J}$ et $\mathbf{B}$. On sait qu'on est obligé d'employer au moins deux verres inégalement réfringents; mais la plupart dentre eux sont diversement dispersifs, c'est-à-dire que les spectres formés par des prismes de même angle ne comportent pas le même rapport des espaces diversement colorés; de sorte que la cö̈ncidence des couleurs $\mathbf{J}$ et $B$ n'assure pas celle des autres. Celles-ci forment alors un spectre secondaire qui altère la pureté des images.

On peut, au point de vue qualificatif, préciser davantage. Les foyers dus aux radiations $\mathbf{J}$ et $\mathrm{B}$ coïncident quand la différence des déviations des rayons est nulle, c'est-à-dire quand on a :

$$
(\mathrm{J}-\mathrm{B}) \boldsymbol{x}+\left(\mathrm{J}^{\prime}-\mathrm{B}^{\prime}\right) \beta=\mathrm{o},
$$

$\alpha, \beta$ étant les angles des prismes déterminés par les plans tangents aux points d'incidence des rayons sur les faces des deux verres.

La coüncidence d'une autre couleur, V par exemple, avec les deux précédentes exigerait que l'on eût :

et par suite :

$$
(\mathrm{J}-\mathrm{V}) \alpha+\left(\mathrm{J}^{\prime}-\mathrm{V}^{\prime}\right) \beta=0,
$$

$$
\mathbf{J}-\mathbf{B}=\frac{\mathbf{J}^{\prime}-\mathbf{B}^{\prime}}{\mathbf{J}-\overline{\mathbf{V}}}=\frac{\mathbf{J}^{\prime}-\overline{\mathbf{V}}^{\prime}}{\mathbf{d}^{\prime}}
$$

Le fait n'a pas lieu; en désignant par $\theta$ le rapport du premier membre de l'équation au second, les tables des indices des verres permettent de s'assurer que $\theta$ est différent de l'unité.

Quant à la déviation du rayon $\mathbf{V}$ par rapport au rayon J, elle est, dans les mêmes conditions d'approximation :

$$
\Delta=(\mathrm{J}-\mathrm{V}) \alpha+\left(\mathrm{J}^{\prime}-\mathrm{V}^{\prime}\right) \beta,
$$


ou, en tenant compte des relations précédentes,

$$
\Delta=(\mathbf{I}-\theta)(\mathbf{J}-\mathbf{V}) \alpha \text {. }
$$

Suivant que 0 sera plus grand ou plus petit que l'unité, le foyer des rayons $V$ se trouvera d'un côté ou de l'autre de celui des rayons $\mathbf{J}$ et $\mathrm{B}$ réunis ( $\Delta$ positif ou négatif).

73. Spectre secondaire. - Ces faits, bien con nus de Clairaut, étudiés après lui par Boscowich, s'étendent, dans leur esprit, à un nombre quelconque de verres constituants. Ie spectre secondaire subsiste. II était à présumer que l'on devait arriver à le faire presque disparaître en employant deux combinaisons de même signe (toutes deux convergentes ou divergentes), dont l'une donnerait à $\theta$ une valeur supérieure à I et l'autre une valeur inférieure. En effet, dans ce cas, la déviation du rayon $V$ aurait pour expression :

$$
(\mathbf{I}-\theta)(\mathbf{J}-\mathbf{V}) \alpha+\left(\mathbf{I}-\theta_{1}\right)\left(\mathbf{J}_{1}-\mathrm{V}_{1}\right) \alpha_{1},
$$

et pourrait être voisine de zéro, car les coefficients $\mathbf{I}-\theta$ et $\mathbf{I}-\theta_{1}$ des quantités de même signe $(\mathrm{J}-\mathrm{V}) \alpha$ et $\left(J_{1}-V_{1}\right) \alpha_{1}$ seraient de signes contraires.

Malheureusement, dans les verres connus à l'époque, la dispersion croissait avec l'indice moyen; $\mathbf{r}-\theta$ avait un signe constant. Le $D^{r}$ Blair $(56)$ eut l'idée de constituer de pareils systèmes en employant des liquides, de compositions variées, contenus dans des lentilles en verre dont le seul rốle était dès lors de servir d'enveloppe. La réussite fut parfaite; mais la solution n'était pas pratique. Les verres obtenus vers 1840 par Guinand auraient conduit au même résultat (21): leur prix de revient, trop élevé, fut un autre obstacle.

La maison Schott, d'Téna, étant parvenue à la fabri- 
cation courante de verres à indice élevé et à faible dispersion, Abbe réalisa, sous le nom d'apochromatique, un objectif de microscope dans lequel le spectre secondaire était éliminé. Basés sur le même principe, des objectifs pholorraphiques apochromatiques furent construits par Steinheil, Zeiss, Harnack, Berthiot (25).

Les combinaisons à deux éléments, indiquées par Blair et réalisées avec des verres, ont reçu de Zciss des noms qu'il nous suffira de signaler ici. Si des deux verres, celui qui a le plus fort indice possède la dispersion la plus grande, la combinaison est dite normale; c'est celle du crown et du flint des objectifs courants de lunette; sinon, elle est dite anomale ou anormale.

74. Correction des aberrations chromatiques. - Io calcul de l'achromatisme Iongitudinal est, devant le problème des aberralions sphériques, relativement simple. Pour deux couleurs, il suffit de remplacer, dans la première des équations $d u \mathrm{n}^{0} 7^{2}$, $\alpha$ et $\beta$ en fonction des éléments géométriques des len tilles. L'équation résultante ne contient que les premières puissances des rayons; on la résout assez aisément par une méthode d'approximation numérique.

Le seul point important est relatif à la précision exigée. La coïncidence des deux foyers ne peut être absolue; la figure ir montre qüil suffit que leur intervalle soit an plus égal à la demi longueur de l'image solide, car un plus grand éloignement réduirait la profondeur de l'image solide quasi-achromatique résultante; un intervalle plus faible n'aurait aucun intérêt, puisque nous supposons identique l'impression due à un point et à l'aire de la base de l'image.

Quant d̀ l'achmomatisme latéral, lorsqu'il n'est pas 
suffiśamment assuré par le précédent (notamment si le champ est considérable), sa réalisation comporte des calculs et des essais pénibles dont il ne peut être question maintenant.

Remarquons, au sujet de l'achromatisme absolu, qu'il semble étrange que, pour faire disparaitre les spectres secondaires, on n'achromatise pas pour trois, quatre, etc., radiations. Mais, alors, on constituerait des systèmes trop complexes, onéreux et très absorbants.

75. Achromatisme apparent. - L'achromatisme apparent est celui des lunettes d'observation; il peut être obtenu presque rigoureusement, quel que soit le nombre de radiations, car il s'agit ici d'une propriété descriptive des images, et non d'une propriété métrique. Biot en a fait une étude approfondlie. Si l'image objective est achromatique, il sera possible de constituer, avec une mème espèce de verre, un oculaire, astronomique ou terrestre, qui assurera la dispersion rectiligne des foyers. Il faudra, en outre, que le point de concours, dit point oculaire, soit en dehors du système optique matériel et à uno distance telle que le centre de la pupille apparente puisse s'y placer. Biot a montré que l'oculaire négatif d'Huygens remplissait ces conditions; mais qu'il n'en était pas ainsi de l'oculaire de la Iunette galiléique, ni de l'oculaire de Ramsden. Ce dernier donne un point oculaire extérieur seulement lorsqu'il est approximativement achromatique.

Dans la pratique, il n'est pas indispensable que le point oculaire soit rigoureusemerıl fixe; il suffit que les droites qui joignent les bords d'une image déterminéc aux bords des autres coupent l'axe en des points suffisamment rapprochés. Les images, d'autre part, ne 
doivent pas être trop éloignées les unes des autres; il faut que l'ceil soit accommodé sur lcur ensemble; mais, de ce côté, la marge est considérable.

Le calcul de l'achromatisme apparent est guidé par des équations asser maniables, mais des tâtonnements sont indispensables.

76. Sous-correction et sur-correction. - Les considérations exposées au $\$ 68$ s'appliquent, dans leur esprit, à l'achromatisme. Si un système doit être corrigé de cette aberration, il sulfit que l'image dernière le soit; les images intermédiaires peuvent être chromatiques; les combinaisons partielles qui les produisent offrent deux caractères différents.

Admettons que le système total doive réunir les couleurs jaune et bleue. L'une des combinaisons pourra laisser le $\mathbf{J}$ au delà du $\mathbf{B}$, elle sera dite sous-achromatisée; l'autre, utilisće seule, ramènerait le jaune en deçà du B, plus qu'il ne faudrait pour l'achromatisme, elle est alors sur-achromatisee.

De même, dans les Iunettes, l'image objective peut ne pas présenter l'achromatisme absolu ; il est possible d'en profiter pour réaliser plus parfaitement l'achromatisme apparent, qui scul importe. Toutefois, le chromatisme dû̀ à l'objectif doil rester faible. "Quoique, en théorie, dit Biot (8), les conditions d'achromatisme ne soient indispensables à remplir que dans l'ensemble de l'appareil, on trouve beaucoup d'avantages à les introduire séparément dans le système objectif et dans le système oculaire. Car, non seulement elles s'obtiennen ainsi, dans l'un et dans l'autre, par des procédés divers qui deviennent plus faciles à réaliser isolément, mais il en résulte, en outre, la possibilité d'adopter successi- 
vement au Inême objectif des oculaires divers qui donnent des grossissements angulaires de grandeurs différentes, sans que l'appareil total cesse de remplir les conditions générales de l'achromatisme, du moins aussi approximativement qu'il est nécessaire pour la netteté de la vision. " 


\section{CHAPIT'RE IV}

DE LA CIARTÉ DES MSTRUMEATS

77. Flux lumineux et notions qui en dérivent. - La notion primitive qu'on rencontre à la base de la photométric est colle d'éclairement, — au sens vulgaire du mot, - d'une surface sur laquelle tombent les rayons d'une source lumineuse. L'ocil, dans certaines conditions, apprécie assez bien l'égalité des éclairements de deux surfaces voisines, et distingue, Ie cas échéant, le sens de leur différence. De cette constatation dérive la notion de l'intensité - ou puissance lumineuse - plus ou moins grande de la source.

Deux sources punctiformes identiques très voisines relativement a la distance d'observation constituent une source unique. Par une double convention, arbitraire, mais logirue, cette dernière source est dite avoir une intensité double de chacune des composantes, et l'éclairement produit sur une surface quelconque est double de celui que produirait l'une des sources primitives, dans les mèmes conditions géométriques et physiques. Qui a lu Euclide peut alors parler de la proportionnalité des intensités des sources aux éclairements qu'elles produisent, du rapport de deux éclairements et du rapport de deux intensités.

L'éclairement produit par une source donnée sur une surface qui se dóplace parallèlement à elle-même est, fait 
expérimental, proportionnel à l'inverse du carré de la distance de la surface à la source ponctuelle. La forme de cette loi conduit, comme dans d'autres branches de la Physique. à la conception du flux et au principe de sa conservation. qui se lie à la notion de transparence du milien acquise d'autre part.

La notion du flux lumineux, sans que celui-ci soit défini, peut ètre considérée dès lors comme la base unique de la photométrie. A cetle grandeur physique, il suffira d'adjoindre des grandeurs géométriques appropriées. Elle peut donner un sens à la quantité de rayons lumineux, sens analogue, non identique, à celui du nombre de lignes de force de Faraday.

Le flux $\Phi$ émanant d'une source punctiforme se répartit intégralement et uniformément sur les différentes sphères concentriques à la source, et se conserve dans des côncs issus de celle-ci. L'intensité I peut donc ètre mesurée comme le llux qui tombe sur l'aire découpée, sur l'une des sphères. par un cone dont l'angle solide est l'unité. Donc .

$$
\mathrm{d}=\mathrm{I} \sigma \text {. }
$$

Ф étant le flux émis dans l'angle solide o par une source ponctuelle d'intensité I.

Ce flux, se conservant dans l'angle solide, donne sur les diverses surfaces qu'il rencontre des éclairements $\mathrm{E}$ inversement proportionnels au carré de la distance, et par suite à l'aire $\mathbf{S}$ découpée, celle-ci étant supposée un corps opaque. De plus, la plupart, sinon tous, des corps naturels opaques possèdent cette propriété expérimentale, que, inclinés diversement sur un faisceau lumineux, ils produisent à l'œil un effet total identique. On peut donc poser dans tous les cas

$$
\Phi=\mathbf{E S} \text {. }
$$

La première relation s'applique à un systène de points lumineux isolés: elle doit être modifiée pour une sounce continue. Un élément superficiel d'aire st lorigine 
d'émission d'un flux qui part de son centre; la partie de ce flux contenu dans l'angle solide unité autour de la normale à l'élément peut évaluer la puissance de cet élément $s$. La notion d'intensité, applicable au point isolé, se transforme ici en celle d'éclat e; la puissance lumineuse de l'élément devient es, et on peut poser

$$
\Phi=e s \sigma .
$$

Il a été nécessaire d'envisager ici lo flux suivant la normale à l'élément, car la puissance lumineuse, - l'œil le constate, - varie avec la direction du flux. La plupart des corps suivent la loi de Lambert : leur puissance est proportionnelle au cosinus de l'angle $\alpha$ formé par la direction du flux a vec la normale à la surface. Donc, plus généralement,

$$
\Phi=e s \tau \cos \alpha \text {. }
$$

Enfin une surface lumineuse d'aire totalc S émet un flux total $\Phi$. Le rayonnement $\mathrm{F}^{\prime}$ intrinsèque de cette source est défini par

$$
\Phi=\mathbf{L} \text { 'S. }
$$

On l'appelle aussi son éclxirement, en raison de la forme de cette relation.

78. Clarté absolue et clarté relative. - Le degré de visibilité d'un objet dépend de l'éclairement, en valeur absolue, de son image rétinienne et du contraste qu'il présente avec le fond sur lequel il se détache. Il en est de même pour une imagre optique.

La règle s’applique aussi à l'image produite par l'objectif photographique : il sulfit de substituer, dans l'énoncé, la plaque sensible à la rétine.

On peut donc distinguer, au moins didactiquement, une clarté absolue et une clarté relative. Dans les appareils où l'ceil intervient, la première sera suffisamment définie par le rapport entre les éclairements rétiniens 
produits par l'image observée à travers l'instrument et par l'objet. Ise même rapport sera utile dans l'étude de l'objectif photographique; mais il servira surtout à comparer les clartés de deux objectifs différents.

Quant à la clarté relative, il suffira généralement d'évaluer si le contraste avec le fond est plus ou moins grand pour l'image que pour l'objet, ou de déterminer les causes qui l'augmentent.

$7^{9}$. Égalité d'éclat de l'image et de l'objet. - Une propriété intéressante est commune à tous les systèmes optiques : l'éclat de l'image a généralement la même valeur que celui de l'objet. Il s'agit, bien entendu, de systèmes parfaitement transparents : la question des pertes de lumière, - de flux, - sera étudiée ensuite.

Considérons (fig. I 2) un système optique (S) don-

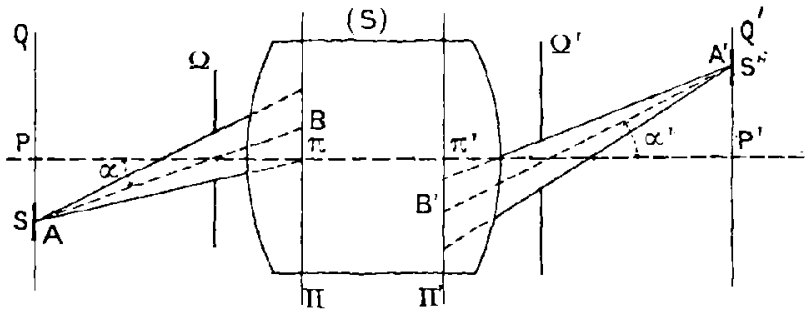

Fig. 12.

nant d'un plan $Q$, normal à son axe, une image plane $Q^{\prime}$; soient $\Omega$ et $\Omega^{\prime}$ les ouvertures respectives d'entrée et de sortie. Le flux utile envoyé par le très petit élément $s$ de Q, situé à une distance angulaire $\alpha$ de l'axe, a pour expression :

$$
\Phi=e s \varsigma \cos \alpha .
$$


En prolongeant (51) le cône qui limite le flux jusqu'au premier plan principal II du systìme, sur Inquel il découpe nne aire w, l'évaluation géométrique de l'angle solide $\sigma$ transforme la relation précédente en :

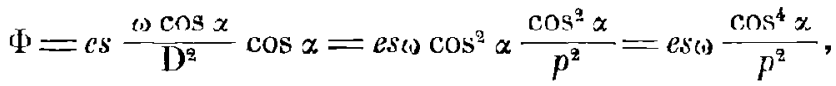
où $\quad \mathrm{D}=\Lambda \mathrm{B}$ et $p=\mathrm{P}_{\pi}$.

Le flux sortant, égal au précédent, a pour expression :

$$
\Phi=e^{\prime} s^{\prime}(1) \frac{\cos ^{4} \alpha^{\prime}}{p^{\prime 2}} .
$$

Car, en vertu même de la définition des plans principaux, les aires découpées par les deux flux sontégales. Donc, on a :

$$
e^{\prime}=e \frac{s}{s^{\prime}} \frac{\cos ^{4} \alpha}{\cos ^{4} \alpha^{\prime}} \frac{p^{\prime 2}}{p^{2}} .
$$

Or on sait que, si $n$ est l'indice du milieu antérieur par rapporl au milieu postérieur, on a :

donc :

$$
\begin{gathered}
\frac{s}{s^{\prime}}=\frac{p^{2}}{p^{\prime z}} \frac{\mathrm{I}}{n^{2}}, \\
e^{\prime}=\frac{\mathrm{I}}{n^{2}} \frac{\cos ^{4} \alpha}{\cos ^{4} \alpha^{\prime}} e .
\end{gathered}
$$

Dans le cas où les milieux extrèmes sont identiques, les deux éclais sont égaux quand l'élément considéré est très voisin de l'axe (vision centrale), ou lorsque, $\alpha^{\prime}=\alpha$, le grossissement angulaire est voisin de l'unité . (cas normal des ohjectifs photographiques pour paysages).

Il pourrait y avoir doute sur la validité de la démonstration quand (cas usuel des lunettes) les plans principaux sont à l'infini ; la continuité géométrique autorise 
cependant l'extension légitine du résultat; la proposition peut d'ailleurs s'ètablir directement.

80. Anneau oculaire. - L'étude de la clarté absolue des lunettes et des microscopes est facilitée par la considération du diaphragme apparent de sortic du système, qui porte dans ce cas le nom d'anneau oculaire ou de cercle de Ramsden. Dans les instruments habituels, cet anneau cst l'image, sensiblement plane et de forme circulaire, de la surface antérieure utile de l'objectif. Le faisceau issu d'un point quelconque du champ de l'instrument et limité par l'ouverture utile passe, à l'émergence, dans l'anneau oculaire dont il couvre entièrement la surface.

Quand l'anneau oculaire, dit alors réel, est situé en dehors de l'instrument, au delà de la surface d'émergence, de manière que la pupille apparente puisse se disposer dans son plan, l'ceil est susceptible, moyennant certains déplacements et dans des conditions particulières d'éclat intrinsèque do l'image, de recevoir tous les rayons émanant d'un point de l'image. Dans ce cas, les rayons émergent de la surface de sortie de l'appareil, en convergeant vers l'anneau oculaire pour diverger ensuite.

Lorsque l'anneau est virluel, c'est-à-dire situé dans le corps de l'instrument, en avant de la surface d'émergence, l'wil ne saurait s'y placer. Ifs rayons sortent divergents du système, leurs prolongements géométriques seuls s'appuyant sur le cercle de Ramsden; l'œil ne peut recevoir la totalité de ceux qui émanent d'un point de l'image, qu'autant que la pupille appareate est au moins égale à la section déterminée par son plan dans le faisceau émergent. 
L'optique gćométrique permet de déterminer aisément, dans chaque cas, la position et le diamètre de l'anneau oculaire. Nous en rappellerons trois résultats.

Les lunettes astronomiques ou terrestres, les télescopes sont généralement à anneau réel. Le diamètre a de celui-ci, en supposant l'objectif mince, est donné par la relation $\Omega=\mathrm{G} a$, dans laquelle $\Omega$ est l'ouverture utile de l'objectif et $G$ le grossissement de l'instrument.

La même formule s'applique à la lunette de Galilée; mais, dans cette combinaison, l'anneau est virtuel.

Dans les microscopes, le cercle de Ramsden est réel, et son diamètre est donné par la relation :

$$
a=\frac{200}{G}
$$

$\omega$ étant l'ouverture numérique de l'objectif, et $\Delta$ la distance de la vision (c'est-à-dire la distance de l'image à la pupille apparente).

8r. Clarté des lunettes à anneau oculaire rèel. - L'éclairement de l'image rétinienne, duquel dépend la clarté absolue d'un instrument où l'oeil est intéressé, est proportionnel au flux reçu par l'wil. Le coefficient de proportionnalité est le même dans la vision directe et dans la vision instrumentale, dans les cas où nous nous plaçons : lunettes rejettant à l'infini l'image d'un objet à l'infini, microscopes réglés pour la distance minimum de la vision distincte. Il s'agit donc d'évaluer les flux reçus par l'oxil dans les deux circonstances : directement, à travers l'instrument.

Nous considérerons d'abord les systèmes à anneau oculaire réel. L'oeil aura tout intérêt à centrer sa 
pupille apparente sur l'anneau; il ne pourrait y avoir hésitation que si le cercle de Ramsden était plus petit que la pupille; mais alors, quoique l'œil puisse recevoir, intégralement, pour diverses positions, les faisceaux émergents, il y a avantage à viser à la coüncidence indiquée, qui permet, sans variation de clarté, la tolérance maximum dans la position de l'œil.

Supposons le centrage effectué ct examinons en premier lieu le cas des lunettes. Le flux direct est donné par $\Phi=k$ e $p^{2}, p$ étant l'ouverture pupillaire. Le flux reçu à travers l'instrument est:

ou

$$
\begin{aligned}
& \Phi^{\prime}=k e^{\prime} p^{2}, \quad \text { si } a>p, \\
& \Phi^{\prime}=k e^{\prime} a^{2}, \quad \text { si } a<p,
\end{aligned}
$$

$a$ étant le diamètre de l'anneau oculaire.

Pour une image centrale, $e^{\prime}=e$, la clarté, rapport des deux flux $\Phi^{\prime} \dot{a} \Phi$, sera l'unité, si l'anneau oculaire est au moins aussi grand que la pupille; l'jmage et l'objet parâ̂tront également éclairés; la clarté sera diminuée dans le rapport de $a^{2} \dot{a} p^{2}$, si la pupille est plus ouverte que l'anneau oculaire.

Pour une image non centrale, la clarté sera, dans tous les cas, encore réduite dans la proportion de $\cos ^{4} \alpha^{\prime}$. Ce coefficient de réduction n'est pas toujours négligeable. Ainsi sur les bords du champ d'une Iunette de grossissement 20 et de $2^{\circ}$ do champ, on a sensiblement $\alpha^{\prime}=20^{\circ}$, et le coefficient en question est 0,78 .

De cette discussion, il résulte que la valeur la plus convcnable à donner au diamètre de l'anneau oculaire est celui de la pupille apparente; mais, cette dernière s'ouvrant d'autant plus que l'objet est moius éclairé 
ou moins lumineux, il s'ensuit que l'anneau devra être calculé pour les conditions d'éclairage les plus mauvaises, eu égrard au rôle de l'instrument.

$8 \mathrm{r}$ bis. Grandeur théorique de l'anneau oculaire. - Cette règle n'est cependant pas parfaite. L'ceil, dans les conditions où nous l'avons placé, ne perçoit distinctement que les points situés à quelques minutes autour de l'axe du système, ceux qui forment l'image rétinienne sur la macula latea. Le champ dans lequel se trouve l'imagre virtuelle, - dit champapparent, - est beaucoup plus étendu; il peut atteindre $20^{\circ}$ autour de l'axe. Or l'cil examine l'image comme un objet réel; il tourne rapidement autour de son centre de rotation. Ce mouvement presque instinctif doil être accompagné, afin que la pupille reste en coüncidence avec l'anneau oculaire, d'un déplacement, plus volontaire et par suite moins rapide, souvent en retard, de la tête de l'observateur. Quand toutes ces variations ne sont pas en concordance, la pupille ne reçoit plus que les rayons tombant sur l'aire, souvent faible, qu'elle a de commun avec l'anneau ocu-

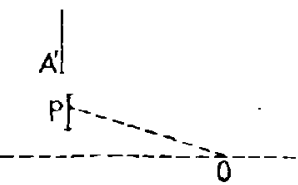

A

Fig. 13. 
pupille d'ouverture $p, \gamma$ le champ réel de l'instrument, $\gamma^{\prime}=\mathrm{G} \gamma$ le champ apparent, la figure indique sans difficulté que l'on doit avoir :

$$
a=\delta \gamma^{\prime}+p=\mathrm{G} \delta \gamma+p \text {. }
$$

En adoptant les valeur's $\delta=1 \mathrm{I}^{\mathrm{m} w}, 2$ et $p=\mathbf{2}^{\mathrm{mm}}, 5$, cette formule donne, pour les caractéristiques suivantes très admissibles :

$$
\begin{array}{lll}
\mathbf{G}=\mathrm{I} 6, & \gamma=0,06 ; & a=\mathrm{I} 2^{\mathrm{mm} \mathrm{m}}, 3 . \\
\mathbf{G}=8, & \gamma=0,08 ; & a=9^{\mathrm{nm} \mathrm{m}} .
\end{array}
$$

Il est malheureusement impossible de satisfaire d̀ ces exigences pour des instruments d'usage courant. Le premier demanderait un objectif de 20 centimètres d'ouverture et, par suite, de 2 mètres à $2^{\mathrm{m}}, 50$ de focale; le second, - dont les données sont celles d'un corps de jumelle de campagne, - devrait avoir un objectif de 72 millimétres d'ouverture et près de 1 mètre de focale. Les conditions d'emploi, le prix de revient s'opposent à la réalisation de ces données.

Il sera avantageux de porter le diamètre de l'anneau oculaire an maximum possible, ne fût-ce que pour éviter à la pupille la nécessité d'un centrage trop pénible. Bien que l'oil se rende compte d'un défaut de concentricité, il lui est difficile de le corriger rapidement s'il. ne perçoit pas une clarté suffisanle. Or, avec un anneau oculaire de $2^{\mathrm{mm}}, 5$, par exemple, un déplacement de la pupille de $\mathrm{o}^{\text {mam }}, 5$ fait perdre le huitième de la clarté de l'image centrale. .

82. Clarté du microscope. - Dans le microscope, l'anneau oculaire est toujours plus faible que lat pupille. Le rapport des clartés de l'inage el de l'objet 
est donc, pour les raisons développées au sujet des lunettes :

$$
\frac{a^{2}}{p^{2}}=\frac{4 \omega^{2} \Delta^{2}}{p^{2} \mathrm{G}^{2}} .
$$

En ouvrant la pupille à 4 millimètres, et supposant $\Delta=250$ millimètres, le rapport ci-dessus deviendrait égal à l'unité, - anneau ouvert comme la pupille, pour :

$$
\mathrm{G}=\mathrm{r} 25 \omega .
$$

Or ce grossissement est beaucoup plus faible que celui qui convient au pouvoir séparateur de l'objectif d'ouverture numérique $\omega$.

Pratiquement, la clarté relative de l'image par rapport à l'objet est sans importance, car l'expérimentateur est. dans une large mesure, maître de l'éclairage.

83. Clar té dans les instruments à anneau oculaire virtuel. - Les phénomènes sont un peu différents quand l'anneau oculaire est virtuel. L'ensemble des faisceaux émergents couvre la pupille $\mathrm{PP}^{\prime}$, même à son maximum d'ouverture; nous le supposons, d'abord; il suffit pour cela que la surface de sortie $\mathbf{S}$ soit assez grande.

Tout faisceau incident tombant sur la surface utile $\Sigma$ d'entrée émerge de façon que son prolongement géométrique couvre l'anneau oculaire $\mathbf{A A}^{\prime}$, mais ces faisccaux émergents sont diversement utilisés.

Menons le cône dont $\mathrm{AA}^{\prime}$ et $\mathrm{PP}^{\prime}$ sont des sections droites (fig. I4); soit $O$ son sommet. Tout faisceau émergent dont le sommet $Q$ sera dans l'angle $\mathrm{AOA}^{\prime}$ atteindra la pupille entière en la débordant. En ne considérant que le cas où l'éclat de l'objet se conserve dans 
l'image, ce qui est le cas des lunettes de Galilée, il en sera de même de l'éclairement rétinien, puisque, soit dans la vision directe, soit dans la vision instrumentale, la même ouverture de pupille est intéressée; par suite, pour tous les points de l'objet correspondants des points $\mathrm{Q}$, la clarté de l'image sera égale à celle de l'objet.

Si le sommet $Q^{\prime}$ du faiscean émergent se trouve dans l'angle $\mathrm{BOB}^{\prime}$, le faisceau entrera entièrement dans la pupille, en y découpant une aire d'autant plus faible

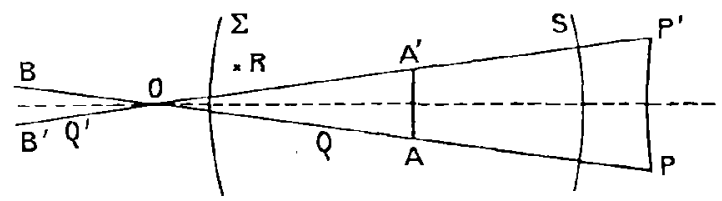

Fig. 1/.

que le point $\mathrm{Q}^{\prime}$ sera plus éloigné de $\mathrm{O}$ et plus rapproché de l'axe du système.

Quand enfin le sommet du faisceau sera en $R$, hors des deux angles précités, une partie excentrée de la pupille se trouvera en dehors du faisceau, et la portion alteinte par ce dernier se trouvera d'autant plus faible que le sommet $\mathrm{R}$ sera plus distant de l'axe et plus loin de l'anneau oculaire.

Dans ces deux cas, $\mathbf{Q}^{\prime}$ et $\mathbf{R}$, la clarté de l'image perçue par l'oeil scra d'autant plus faible que l'aire intéressće de la pupille sera plus réduite.

Il suit de là que, pour un objet uniformément éclairé, si la mise au point se fait entre $O$ et l'anneau oculaire, on percevra un cercle uniformément éclairé, entouré

Instruments optiques. 
d'une couronne dont l'éclairement décroîtra en s'éloignaut du centre; si la mise au point a lieu au delà de $O$, la couronne extérieure présentera le même caractère; mais, dans ce cercle intérieur, l'intensité décrồtra en allant de la circonférence au centre.

La seule conséquence que nous en tirerons ici est que, le premier aspect étant évidemment meilleur, il sera utile de l'obtenir dans tous les cas, et par suite, rejetant $\mathrm{O}$. au moins à l'infini, de donner à l'anneau oculaire une ouverture au moins égale à celle de la pupille maxinum.

Les mêmes apparences se prćsenteront si, contrairement à ce que nous avons admis, la pupille ne recevait pas entièrement le faisceau total émergent; mais en outre le champ entier serait uniformément assombri dans le rapport des aires de la pupille active et de la pupille totale. Il est donc indispensable que la surface de sortie ail une dimension suffisante, d'ailleurs aisée à déterminer.

Ces considérations seront appliquées plus loin à la lunette de Galilée.

84. Éclat des images ponctuelles. - Nous avons implicitement admis jusqu'ici que l'objet examiné était de dimensions finies; dans cette hypothèse, les phénomènes de diffraction n'ont qu'une influence, d'ailleurs négligeable, sur les bords de l'image. Il n'en est plus de même quand on regarde un point lumineux isolé, par exemple une étoile. Le cas doit être traité à part.

Une étoile, vue à l'œil nu, donne une image rétinienne de diamètre $\alpha$, qui produit la sensation d'un point. l'éclairement de cette image est le rapport de $\Phi=k e p^{2} \quad \dot{\mathrm{a}} \quad \alpha^{2}$. 
Le flux $k e \Omega^{2}$ reçu par la lunette, et que nous supposons intígralement transmis, se répartit suivant une Joi connue sur la tache centrale et ses différents anneaux. Quand l'ceil peut percevoir tout cet eusemble, - le fait dépend du grossissement de la lunette et de l'éclat intrinsèque de l'étoile, - il est difficile de précisẹr ce qu'on doit entendre par éclat de l'image. Admettons, comme corollaire plausible de la loi de Hooke, que ce soit l'éclairement spécifique moyen sur l'étendue $\triangle$ de la partie de l'image vue de l'ceil sous l'angle de $\mathrm{I}^{\prime}$ : cette hypothèso dispense de tenir compte de la valeur de $\alpha$.

Le rayon $\sigma$, qui, sur l'image objective, correspond à la portion $\Sigma$ de l'image virtuelle, est donné par la relation :

$$
\sigma=\frac{60 \Omega}{\mathrm{I} 3 \mathrm{OG}} p=u_{\rho} \text {. }
$$

Le flux réparti sur Sest une fraction, $\Phi^{\prime}=k e \Omega^{2} \mathrm{~B}^{2}(u)$, du flux reçu; B est une fonction connue (4).

Lorsque, - c'est le cas le plus commun, - la pupille est plus ouverte que l'anneau oculaire, ce flux pénètre entièrement dans l'coil, se répartissant sur l'image rétinienne. $\alpha$, le rapport des éclats de l'image et de l'objet est celui de $\Phi^{\prime}$ à $\Phi$, c'est-̀̀-dire :

$$
\mathrm{R}=\left[\frac{\Omega}{p} \mathrm{~B}(u)\right]^{2} \text {. }
$$

Si $u \geq \mathrm{I}$, et par suite si le grossissement est au plus égal au grossissement normal, B (u) est sensibleInent égal à l'unité; l'image paraît alors plus brillante que l'étoile, car, en pratique, l'objectif $\Omega$ est plus 
ouvert que la pupille. D'ailleurs, dans cette circonstance, la sensation rétinienne est ponctuelle.

A mesure que le grossissement augmente à partir de sa valeur normale, $u$ et, par suite, B $(u)$ diminuent. Il en est, par suite, de même de l'éclat relatif $R$, qui peut, pour des grossissements ólevís, devenir très inférieur à l'unité. L'expérience vérifie cette conclusion : les étoiles observées à l'aide de lunettes très grossissantes paraissent moins brillantes qu'à l'œil nu.

I.es mêmes conclusions s'appliquent quand l'anneau oculaire est inférieur à la pupille; mais alors $\mathrm{R}$ doit être nultiplić par le rapport de $p^{2}$ à $a^{2}$; en conséquence, dans son expression, $p$ doit être remplacé par $a$.

85. Clarté de l'objectif photographique.

L'évaluation de la clarté des photo-abjectifs est une conséquence immédiate de la formule établie au $\mathbf{n}^{\circ}{ }_{7} 9$, relative au flux entrant. Ce flux, se répartissant sur l'aire $s^{\prime}$, donne à l'image un éclairement :

$$
\begin{aligned}
\mathrm{E}^{\prime}=\frac{\Phi}{s^{\prime}}=e \frac{s}{s^{\prime}} & \frac{\omega}{p^{2}} \cos ^{4} \alpha=e \frac{s}{s^{\prime}} \frac{p^{\prime 2}}{p^{2}} \frac{\omega}{p^{\prime 2}} \cos ^{4} \alpha \\
& =e \frac{\omega}{p^{\prime 2}} \frac{\cos ^{4} \alpha}{n^{2}} .
\end{aligned}
$$

Et comme, en général, $n=x$, on $a$, en appelant $\Omega$ le diamètre de (1) :

$$
\mathrm{E}^{\prime}=\frac{\mathrm{I}}{4} \pi e\left(\frac{\Omega}{p^{\prime}}\right)^{2} \cos ^{4} \alpha .
$$

C'est l'éclairement $\mathrm{E}^{\prime}$ qui détermine l'intensité de l'image photographique, et non l'éclat $e^{\prime}$; car l'impression lumineuse sur la plaque croît évidemment avec l'ouverture du cône de flux qui arrive sur un de ses points. 
La qualité d'un objectif photographique, au point de vue de la clarté, dépend du coefficient de $e$ dans l'expression ci-dessus. Pour la caractériser par un nombre unique, on a l'habitude de faire $\alpha=0$ et $p=\infty$, c'est-̀̀-dire de supposer un petit objet à l'infini, sur l'axe. La clarté est proportionnelle au carré du rapport de $\Omega$ à la focale principale

$$
\mathrm{r}=h\left(\frac{\Omega}{\mathrm{F}}\right)^{2} \text {. }
$$

On détermine $h$, dans l'usage courant, en supposant $\Gamma=h$ pour $\frac{\Omega}{\mathrm{F}}=\frac{1}{10}$, d'où :

$$
\Gamma=\mathrm{s} 00\left(\frac{\Omega}{\mathrm{F}}\right)^{2} \text {. }
$$

La clarté d'un objectif pholographique ne dépend que du rapport de son ouverture à sa focale. Cette propriété explique et justifie la désignation, adoptée par -les constructeurs, de la qualité de l'objectif, par ce rapport, mis généralement sous la forme $\frac{I}{m}$. Mais il faut insister sur ce point que, si on prend pour $\mathrm{F}$ la focale principale, $\Omega$ doit être la section par le plan principal postérieur du cône de sortie tombant sur le foyer, et non l'ouverture réelle ou apparente du diaphragme.

86. Pertes par réflexion. - Aucun instrument n'étant parfait, le flux sortant est toujours, en réalité, pluş faible que le flux entrant. La différence, perte dans le système, provient des réflexions sur les surfaces réfringentes, de l'absorption par les masses réfringentes dans leur traversée, de l'absorption et de la dif- 
fusion par les surfaces réfléchissantes dont le poli n'est pas idéal, et enfin de la réfringence des surfaces qui doivent agir soit par réflexion tọtale, soit par réflexion partielle en proportion déterminée.

En décomposant virtuellement un faisceau de lumière riaturelle en deux faisceaux polarisés à angle dmit, Fresnel a pu évaluer la proportion $p$ de lumière inci-

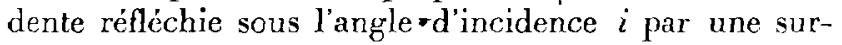
face parfaitement transparente et polie :

$$
p=\frac{\mathrm{I}}{2}\left[\frac{\sin ^{2}(i-r)}{\sin ^{2}(i+r)}+\frac{\operatorname{tg}^{2}(i-r)}{\operatorname{tg}^{2}(i+r)}\right],
$$

Les hypothèses admises par Fresnel sont suffisamment justifiées pour le verre. La relation ci-dessus peut donc être adoptée.

Pour avoir une idée de la perte par réflexion, - que la formule permet d'ailleurs de calculer exactement dans tous les cas, - supposons l'indice du verre égal a $I, 5$ et considérons le faisceau incident, successivement dans l'air et dans le verre : soit d'abord $\sin i=n \sin r$, puis $\sin i=\frac{\mathbf{I}}{n}-\sin r$.

La proportion $p$ de lumière rélléchie est, dans chaque cas, une fonction de l'angle d'incidence qu'on peut traduire par les courbes ci-contre (fig. i5) en coordonnées rectangulaires. Ia forme de ces courbes montre qu'il commence à être désavantageux d'avoir des angles d'incidence supérieurs à $35^{\circ}$ sur le verre (traits pleins) et à $65^{\circ}$ sur l'air (traits pointillés). C'est pour cette raison qu'on évite, dans la mesure du possible, de faire attaquer les surfaces des lentilles des lunettes sous des angles supérieurs à $30^{\circ}$. Jusqu'à cette limite, la perte 
n'est guère plus sensible que sous l'incidence normale; mais elle croít ensuite très rapidement, surtout si le rayon doit passer dans l'air.

Certaines surfaces intérieures des objectifs photographiques doivent avoir, en vue de la correction des aberrations, des courbures qui n'admettraient pas tous les faisceaux sous des incidences assez faibles; et comme la perte de lumière s'opère, si faible que soit l'épaisseur de la lame d'air séparant deux lentilles, on se trouve obligé, malgré

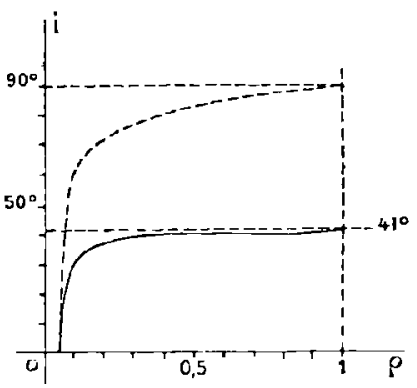

Fig. 15. les inconvénients d'un autre ordre qui en résultent, de coller les diverses lentilles d'une combinaison, c'est-à-dire de les réunir par un milieu d'indice voisin de ceux des verres.

87. Pertes par absorption. - La perte par absorption qu'ćprouve un flux lumincux parallèle $\Phi$ est, par un principe acceptable, proportionnelle à $\Phi$ et à l'épaisseur traversée, lorsque celle-ci est infiniment petite. La traduction de cette proposition est l'équation différentielle

$$
-d \Phi=k \Phi d x
$$

dont l'intégration donne pour le flux $\Phi$ ' ayant traversé l'épaisseur finie $u$ :

$$
\Phi^{\prime}=\Phi a^{u},
$$

a étanl une constante d'absorption dépendant de la nature du milieu et de celle des radiations transmises. 
Pour le crown et le flint ordinaires, $a$ est voisin de 0,985 quand $u$ est exprimé en centimètres $(80)$. Pour les autres verres, il est très variable, et des indications sur ce sujet ne pourraient être utiles quà la condition d'être très détaillées.

88. Pertes par diffusion. - La perte par absorption des surfaces opaques imparfaitement polies ne peut être appréciée qu'expérimentalement. Il semble que, pour les métaux bien polis, on puisse admettre un taux de so $\%$. Comme la réflexion spéculaire importe seule avec ces surfaces, il faut compter en outre la perte par diffusion, qui peut être évalućc à $40 \%$ pour les miroirs mélalliques, à $30 \%$ pour les glaces en verre argentées ou étamées, et descendre à $5 \%$ pour une couche argentée déposée sur une surface de verre poli, puis brunie et soigneusement polie. Mais .ces nombres sont susceptibles de larges variations, en raison des dégradations accidentelles, difficiles à éviter en toutes circonstances, qui peuvent survenir au miroir : oxydation des métaux, désagrégation de la couche ou sulfuration de l'argent.

89. Pertes par réfraction. - Dans certains instruments, les prismes de verre, agissant par réflexion totale, remplacent avantageusement les miroirs à couche réfléchissante métallique. La perte de lumière, par l'action propre de la surface, est évitée; subsiste celle due à l'absorption de réfringence. Mais le phénomène de la réflexion totale suppose la conservation absolue de la différence entre l'indice du verre et l'air environnant. Qu'un souffle de buée se dépose sur la surface, qu'une couche imperceptible de graisse s'étende sur elle, l'indice du milieu passe de la valeur I à la valeur de I.33 
(eau) on r,/77 (glycérine, huiles), trop voisin des indices ( $\mathrm{r}, 49$ à $\mathrm{r}, 60$ ) des verres : séuls se réfléchissent alors les rayons très inclinés sur la surface, et non ceux pour lesquels le prisme était prévu. Ces derniers sont partiellement transmis; la formule de Fresnel permet d'en calculer la proportion; en tous cas, la clarté de l'image diminue, souvent considérablement.

Le même phénoméne se présente avec les prismes à réflexion partielle.

9o. Clarté relative; illumination du champ. - Une image ne pourrait être perçue si, avec la même coloration, elle présentait mềne clarlé que le fond. Toute cause qui éloignera de l'unité le rapport de ces éclairements fera croître la clarté relative.

Si, à l'éclairement normal de l'imagre et du fond, on superpose, dans un instrument quelconque, une illumination générale du champ, la clarlé relative diminuera. Le système optique pent y contribuer de lui-même par son organisa-

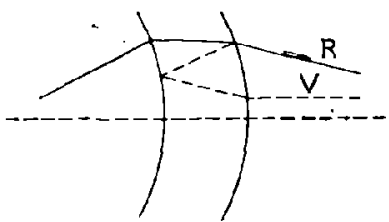

Fig. 16. tion; en plus (fig. I6) de l’image normale due uniquement à la réfraction $R$, il peut s'en former une ou plusieurs autres à la suite de réflexions $V$, au nombre de deux au moins, la seconde ayant lieu sur une surface antérieure à la première. Sans doute, ni l'oeil ni la plaque, suivant le cas, ne sont nécessairement au point sur cette image; mais Ies rayons qui la constituent n'en continuent pas moins leur route pour impressionner plus ou moins confusément l'or- 
gane récepteur. Cette apparence constitue la tache centrale des objectifs photographiques. On l'évite, avec ces systèmes, en reportant en avant des différentes surfaces actives les surfaces apparentes de sortie, - images du diaphragme, - correspondant aux divers modes de róflexions et de réfractions combinés, c'est-à-dire en rendant ces surfaces apparentes virtuelles. On ne peut y parvenir d'ailleurs que par un choix convenable des rayons des verres.

I'illumination peut provenir des ohjets situés en dehors du champ : les rayons qui en ćmanent n'arrivent pas directement sur l'orgrane récepteur; mais ils peuvent y parvenir, plus ou moins affaiblis, à la suite de réflexions sur les parois intérieures de la monture do l'instrument ou sur los poussières déposées sur les verres (sur la surface d'entrée, principalement). On arrête ces rayons nuisibles en munissant les instruments de pare-soleils (lunettes et photo-objectifs), qui abritent surtout la surface d'entrée, en noircissant soigneusement et au ton mat toutes les surfaces intérieures non actives, el, dans les appareils longs (lunettes et microscopes), en disposant dos diaphragmes spéciaux, - dits de clarté, - pour arrêter les rayons réfléchis ou diffusés par les parois intérieures.

Au même ordre d'idées se rapporte l'emploi d'cilletons, d'cillères ou de bonnettes d'oculaire, de protecteurs mobiles ou non pour l'œil, dont le rôle est d'éviter l'illumination directe du champ rétinien par les objets extérieurs à l'instrument. Le noircissage mat des cilletons de lunettes s'impose, lorsqu'ils n'emboîtent pas l'wil ; sinon les réflexions sur Jeur surface occasionnent une perte de clarté relative, souvent considérable. 


\section{9r. Variation du contraste avec le champ.} - La clarté relative, quand on passe de la vision directe a la vision instrumentale, ne peut augmenter que lorsque s'atlaiblit la clarté du fond sur lequel se détache l'image, si l'objet est plus éclairé que le fond; quand l'objet est plus sombre que le tableau, tout affaiblissement de clarté de celui-ci entraîne une porte de clarté relative. Il ne saurait d'ailleurs être question d'agir sur la clarté absolue de l'image, qu'on est maître seulement de diminuer.

Par suite de la réduction de leur champ, les lunettes montrent les objets très lumineux mieux éclairés qu'à l'oeil nu.

Une étoile, objet punctiforme, possède le même éclate à l'œil nu ou à l'oil armé d'une lunette de grossissement inférieur au grossissement normal. A l'weil nu, le champ rétinien acquiert un éclairement qui provient d'une région angulaire $\alpha$ d'éclairement moyen $\varepsilon$ autour de l'étoile. Il est difficile de préciser cette valeur de $\alpha$, tant à cause de la mobilité de l'oil que de l'ignarance des circonstances exactes qui amènent une partie du flux émis sur la tache diffractée; mais $\alpha$ doit ètre assez grand, comme on le verra ci-dessous. A travers l'instrument, l'éclairement $\varepsilon$ se conserve; mais te fond actif se restreint à une valeur angulaire au plus égale à la moitié du champ $\gamma$ de l'appareil. Le rapport des clartés relatives est donc:

$$
p=\frac{\mathrm{G}^{2} e}{e}: \frac{\frac{1}{1}^{-\varepsilon \gamma^{2}}}{\varepsilon \alpha^{2}}=\frac{4 \mathrm{G}^{2} \alpha^{2}}{\gamma^{2}} .
$$

Que $2 \alpha$ soit considérable envers $\gamma$, le fait est connu : en - observant, en plein jour, le ciel à̀ travers un long tuyau 
$(G=1)$, on peut distinguer les étoiles; donc $p>1$, et $2 z>\%$. Il s'ensuit que $\rho$ sera d'autant plus éloigné de l'unité que le grossissement $G$ sera plus élevé.

Il peut cependant être nécessaire de modifier cette conclusion quand la pupille est plus ouverte que l'anneau oculaire, et, surtout, quand le grossissement G devient trop grand. On utilisera, le cas échéant, la même méthode, cn tenant compte des considérations développées antéricurement (84).

Lorsqu'il s'agit, non d'une étoile, mais d'un objet de diamètre apparent sensible, les éclairements de l'objet et du fond se conservent; si alors, $\omega$ est la grandeur apparente de l'objet à l'œil nu, on a :

$$
\rho=\frac{G^{2} \omega^{2}}{\omega^{2}}: \frac{-\frac{1}{4} \varepsilon \gamma^{2}}{s \alpha^{2}}=\frac{4 G^{2} \alpha^{2}}{\gamma^{2}} .
$$

Identique à la précédente, cette formule comporte les mêmes conclusions.

92. Remarques générales sur la clarté des instruments. - De l'étude qui précède, il ressort que la clarté relative des instruments dépend, surtout, de leur organisation mécanique. Quant à leur clarté absolue, elle peut être caractérisc̉e assez simplement, si toutefois on veut bien tenir compte des conditions spéciales dans lesquelles l'évaluation a lieu. Le diamètre de l'anneau oculaire, quand il est réel, donne une idée assez juste de la clarté de l'instrument; c'est l'aire de ce cercle que les constructeurs indiquent à cel effet. Si l'anneau est virtuel, l'ouverture de la surface réelle de sortie doit être suffisante; toute autre condition est inutile. Enfin, pour l'objectif photographique, le taux 
du travail, - rapport de l'ouverture efficace à la focale, - est une donnée qui suffit dans bien des circonstances.

Il faut cependant, dans tous les cas, que les pertes de clarté par absorption ou par réflexions inutiles soient négligeables; l'examen ne justifie pas toujours cette hypothèse, et qui se baserait uniquement sur l'ouverture de l'anneau oculaire pour apprécier la clarté d'une lunette pourrait se tromper grossièrement.

Enfin, une remarque doit être faite ici au sujet de l'objectif photographique. Il y a pour lui deux sortes de clartés : la clarté visuelle ou optique, due aux radiations jaune et verte, les plus sensibles à l'veil; et la clarté actinique, qui provient des rayons plus réfrangibles. Elles ne sont pas proportionnelles. Tel objectif donnant une image très claire à l'wil pourra être médiocre comme rapidité d'exposition. La plupart des verres, non tous, absorbent proportionnellement plus de lumière bleue que de lumière jaune; les flints qui jaunissent ou se tachent n'altèrent guèrẹ l'image visuelle, et, pourtant, affaiblissent. l'effet actinique. Aussi faut-il n'accepter que sous réserves les essais photométriques d'objectifs photographiques, où l'œil seul intervient dans les conditions habituelles de la vision.

Instruments optiques.

IRIS - LILLIAD - Université Lille 1 


\section{CIIAPITRE V}

\section{CIIAMP DES IYSTRGMEYTS}

93. Limitation du champ; diaphragmes. Tout instrument est constitué par des éléments optiques (lentilles, miroirs, prismes) centrós sur un même axe. Les conditions optiques, mécaniques, économiques, tenant à l'emploi prévu de l'appareil imposent, entre certaines limites, à ces éléments des dimensions, des formes et des intervalles.

- Un faisceau (fig. I7) qui a traversé in tégralement une

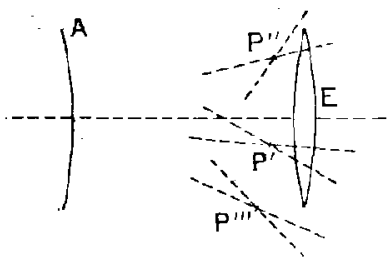

Fig. 17. partie $\Lambda$ du système peut, dans sa route ultérieure, venir passcr en dehors d'un élément subséquent E. Le faisceau est dès lors arrêté, et le point $\mathrm{P}$ correspondant à l'image $\mathrm{P}^{\prime \prime \prime}$ est en dehors du chanup réel.

Quand le faisccau réfracté de sommet $\mathrm{P}^{\prime}$ tombe intégralement sur l'élément, et qu'il possc̀de le même caractère dans le reste du système, le point conjugué $\mathrm{P}$ 
est évidemment dans le champ réel : on ne peut exiger, a priori, d'autre condition à remplir.

Enfin, si dans son parcours le faisccau, tel celui qui a son sommeten $\mathrm{P}^{\prime \prime}$, ne tombe que partiellement sur un ou plusieurs éléments $\mathrm{E}$, le point objet correspondant pourra être aperçu par l'organe récepteur ; mais tous ces points formeront, autour du champ de visibilité maximum, une zone, en grénéral étroite, où la clarté sera moindre et en outre très variable. On évite l'aspect désagréable de cette zone en la supprimant : on arrête systématiquement, à l'aide de diaphragmes, tous les faisceaux, tels que $\mathrm{P}^{\prime \prime}$, qui n'atteignent pas totalement tous les éléments optiques du système.

Ces diaphragmes, dit de champ, - car ils limitent matériellement celui-ci, - ne peuvent être disposés que dans les plans où se forment des images réelles. Con seul suffirait dans chaque instrument; il est prudent d'en placer, cependant, à chaque image réelle. Leurs ouvertures doivent être dans un rapport convenable, déterminé par ce fait qu'elles sont des images les unes des autres. En dehors de ces diaphragmes, le champ peut être encore borné par les diaphragmes de limitation, ouvertures des verres comprises. Les diaphragmes de clarté n'ont aucune influence sur l'amplitude du champ.

94. Champ réel ; champ apparent. - Le champ réel est la région de l'espace, supposé invariablement lié à l'instrument, dans laquelle sc trouvent les objets perçus par le système optique; on donne le nom de champ apparent à la région dans laquelle se forment les images.

Le champ angulaire apparent $\Gamma$ d'un instrument de 
grossissement angulaire $G$ est évidemment lié au champ angulaire réel $\gamma$ par la relation ${ }^{1}$ :

$$
\mathrm{r}=-\mathrm{G} \% \text {. }
$$

La considération du champ apparent facilite l'étude de certaines questions; $c_{n}$ outre, dans les appareils à oculaire, sa valeur caractérise assez exactement la qualité optique de l'oculaire.

On l'appelle souvent encore champ amplifié : la relation précédente explique cette dénomination.

95. Amplitude du champ en fonction des diaphragmes. - La présence de diaphragmes de champ suppose au moins

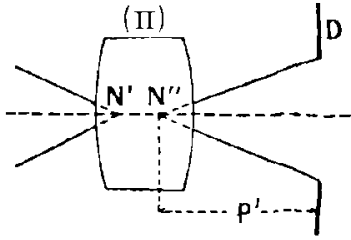

Fig- 18. une image réelle. C'est le cas des lunettes astronomiques ou terrestres et des microscopes. D'une manière générale (fig. I 8), quelle que soit la portion $(\pi)$ du système total (S) qui donne l'image dans le plan du diaphragme D d'ouverture $\delta$, le chanı réel $\gamma$ est sensiblement déterminé par ( $\mathrm{r}$ )

$$
\gamma=2 \operatorname{arctg} \frac{\delta}{2 p^{\prime}},
$$

$\mathrm{N}^{\prime}$ et $\mathrm{N}^{\prime \prime}$ étant les points nodaux.

1 Nous employons, en maintes circonstances, des formules approchées. On rétablira aisèment les formules plus exactes. Ici les champs sont supposés faibles. Sinon, on aurait, avec un grossissement constant, et les champs mesurés en fractions du cercle :

$$
\Gamma=2 \operatorname{arctg}\left(\mathrm{G} \operatorname{tg} \frac{\gamma}{2}\right) \text {. }
$$


Il est donc variable avec la distance du point objet. Dans les lunettes, le champ est évalué pour un objet à l'infini; donc $p^{\prime}=\mathrm{F}$, focale principale de $(\pi)$. Si, de plus, l'objectif est mince, que l'on considère la première image réelle et si l'angle $\gamma$ est assez faible, on obtient la relation approchée ${ }^{1}$ :

$$
r=\frac{\delta}{\mathbf{F}},
$$

F étant la focale de l'objectif, dans lequel les points nodaux sont confondus avec le centre optique.

Lorsqu'on connaît l'ouverture $\delta^{\prime}$ du diaphragme d'une autre image réelle, on pourra soit calculer F et appliquer la formule grénérale, soit construire l'image de $\delta^{\prime}$ à travers les verres placés entre $\delta^{\prime}$ et l'objectif (verre de champ dans la lunette astronomique avec oculaire d'Huygens, véhicule et verre de champ dans la longuevue terrestre); l'ouverture de cette image sera $\delta$, et la dernière formule écrite s'appliquera.

La formule ( $\mathrm{I}$ ) devra être utilisée pour le microscope; des considérations géométriques simples détermineront ensuite le champ linéaire, quand le champ angulaire sera connu.

Le champ apparent se calcule par la formule

$$
\mathrm{T}=2 \operatorname{arctg} \frac{\delta}{2 p^{\prime \prime}},
$$

$p^{\prime \prime}$ étant la distance du diaphragme au point nodal d'émergence de la partie arrière $\left(\pi^{\prime}\right)$ du système total.

Il peut être parfois plus simple de calculer le champ

I On aurait une formule plus exacte en considérant les ouvertures apparentes d'entrée et de sortic; mais cèlle-ci cst, en Eénéral, suffisante. 
apparent, et d'en déduire ensuite le champ réel : ainsi dans une lunette astronomique avec oculaire négatif, si le verre d'ocil, supposé mince, a une focale $f^{\prime \prime}$, on aura :

$$
\Gamma=\frac{\delta}{f^{\prime}}, \quad \gamma=-\frac{\delta}{G f^{\prime}} .
$$

Dans tous les instruments à diaphragmes de champ effectifs, l'uniformité de clarté des champs sera réalisée dans la mesure du possible; en d'autres termes, si le champ réel est uniformément éclairé, il en sera de mème du champ apparent.

$9^{5}$ bis. - Soient, maintenant, D un diaphragme de limitation et (fig. I9) $\mathrm{D}^{\prime}$ son

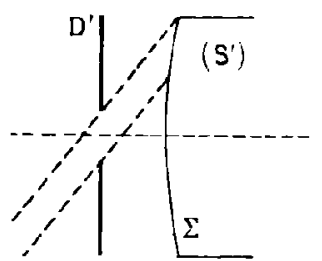

Fig. 19. image par rapport au système parliel antérieur $\left(\mathbf{S}^{\prime}\right)$; si $\Omega$, $\omega$ sont respectivement l'ouverture de la première surface $\Sigma$ du système et celle de $\mathrm{D}^{\prime}$, le champ possible est évidemment donné par la relation très approchée ( $\Sigma$ est supposée plane) :

$$
r= \pm \Omega-\omega \text {, }
$$

¿ étant l'intervalle de $\mathrm{D}^{\prime}$ à $\Sigma$.

Cette relation concerne des objets situés à l'infini (faisceaux parallèles); elle est aisée à généraliser. Le signe du second membre doit être choisi de façon que $\gamma$ soit positif.

Si on établit les relations analogues pour tous les diaphragmes, y compris les ouvertures des verres, ou plus rigoureusement pour toutes les sections droites 
transparentes de l'instrument, - on obtiendra un certain nombre de valeurs telles que $\gamma$ (théoriquement, une infinité ; pratiquement, quelques-unes). La plus faible d'entre elles donnera la valeur du champ réel.

Si dans la relation ( $\mathrm{r}$ ) ci-dessus, le signe + s'impose, $\Sigma$ ne travaillera, pour chaque faisceáu, que sur une partie convenable de son étendue; sinon, tous les faisceaux utiles atteindront la surface entière de $\Sigma$, mais passeront par des régions diverses de $\mathrm{D}^{\prime}$.

Lorsqu'au lieu de $\Sigma$, on considère la surface de sortie, et que les images des diaphragmes sont formées relativement ḋ la partie postérieure du système, les formules telles que (I) se, rapportent au champ apparent.

La formule (I) concerne le champ d'égale clarté, celui des points $\mathrm{P}^{\prime}$ de la figure $\mathbf{1} 7$. Le champ total des points $\mathrm{P}^{\prime}$ et $\mathrm{P}^{\prime \prime}$ est donné par

$$
\gamma^{\prime}=\frac{\Omega+(1)}{\dot{\partial}} .
$$

$9^{6 .}$ Champ de la lunette de Galilée. - Dans un instrument ne donnant aucune image réelle, la limitation du champ résulte exclusivement de celles des surfaces réfringentes; on ne saurait annuler cette zone, entourant le champ de clarté maximum, que, ailleurs, le diaphragme de champ fait disparaître.

Considéré comme formant un ensemble avec l'wil, un instrument de cette espèce comporte comme diaphragme de limitation la pupille. Ios formules indiquées ci-dessus s'appliquent à ces systèmes. Pour eux, le champ total comprend donc un champ de pleine lumière et un champ d'éclairement variable. Le premier, 
seul véritablement utilisable, pourra être comparé au champ des appareils de même emploi, mais à diaphragmes réels; le sccond ne sera guère employé que comme champ de recherche, et non d'observation.

On obtient des résultats simples pour la lunette de Galilée, qui fait partie de cette classe d'instruments.

Jous supposons (fig. 20) l'objectif $\Omega$ et l'oculaire $\omega$

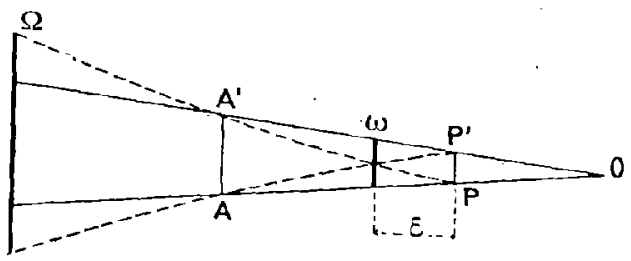

Fig. 20.

infiniment minces et leurs foyers en coïncidence; comme il s'agit en outre de la vision à l'infini, nous admettrons que le point $O$ est en arrière de l'oculaire (83), ce qui revient à supposer $a>p\left(\mathrm{AA}^{\prime}>\mathrm{PP}^{\prime}\right)$.

Tout point situé dans l'angle $\mathrm{AOA}^{\prime}$ appartient au champ amplifié $\Gamma$ de ploine lumière. Donc, en supposant les angles faibles,

$$
\mathrm{I}_{0}=\frac{a-p}{n+\varepsilon}=\frac{[\Omega-G p] \mathrm{G}}{\mathrm{G}^{\alpha} \varepsilon+(\mathrm{G}-\mathrm{I}) \mathrm{F}},
$$

$\operatorname{car} \quad \frac{\mathrm{l}}{\mathrm{n}}-\frac{\mathrm{I}}{\mathrm{F}-f}=\frac{\mathrm{x}}{f} \quad$ et $\quad \mathrm{G} a=\Omega, \quad \mathrm{F}=\mathrm{G} f$

Le champ réel correspondant sera donc:

$$
\gamma_{0}=\frac{\Omega-G p}{G^{q} \varepsilon+(G-\mathbf{G}) F^{\top}} .
$$

Ce champ, où la clarté de l'image se conserve 
(83), croit avec l'ouverture $\Omega$ de l'objectif et diminue quand la pupille s'ouvre. A remarquer que, dans les lunettes ordinaires, le champ est constant.

Pour qu'un point en dehors du champ apparent $\mathrm{I}_{0}$ envoie quelque rayon à la pupille, il suffit qu'il soit dans l'angle du cône s'appuyant sur $\mathbf{A A}^{\prime}$ et sur $\mathrm{PP}^{\prime}$ et de sommet intćricur. Le champ amplifić total $\Gamma$ peut donc s'exprimer par :

$$
\mathrm{I}=\frac{a+p}{n+\varepsilon}=\frac{[Q+\mathrm{G} p] \mathrm{G}}{\mathrm{G}^{2} \varepsilon+(\mathrm{G}-\mathrm{I}) \mathrm{F}},
$$

et par suite le champ réel total est :

$$
\gamma=\frac{\Omega+\mathrm{G} p}{\mathrm{G}^{2} \varepsilon_{\mathrm{r}}+(\mathrm{G}-\mathrm{I}) \mathrm{F}} .
$$

Ce champ total, champ intégral de recherche, augmente avec l'ouverture de l'objectif et de la pupille. Cette remarque est à retenir dans l'emploi de la lunette de Galilée comme instrument de découverte.

Il y a intérêt à considérer le rapport du champ de pleine lumière au champ total. Son expression est:

$$
p=\frac{\Omega-G p}{\Omega+G p} .
$$

Ce rapport ne peut être égal à l'unité; par suite, la zone d'éclairement variable existe toujours, el croì à mesure que le champ réel est moins éclairé, et qu'en outre le grossissement s'élève. Quant à la clarté de la zone, elle décrô̂t progressivement en allant du bord intérieur au bord extérieur.

\section{Champ de l'objectif photographique. -} Le champ de l'objectif photographique est limité par les mêmes causes que celui des autres systèmes optiques; 
mais comme, sauf certaines combinaisons spéciales, le photo-objectif ne donne qu'une seule image réelle, il n'y a pas à proprement parler de diaphragme de champ; seul mériterait ce nom le châssis ou l'intermédiaire qui porte la plaque sensible. Ies diaphragmes dont sont munis les objectifs sont des diaphragmes de limitation.

Le champ, matériellement limité par eux ou par les montures des verres, acquerrait des valeurs considérables, surtout dans les objectifs ramassés suivant l'axe, si des conditions de clarté et de netteté des images n'obligeaient à le restreindre de parti pris. Ia question de la clarté a déjà été suffisamment traitée; celle de la netteté est commune à tous les systèmes optiques.

98. Champ net; courbure du champ. - Il ne suffit pas, pour qu'un point puisse être considéré comme faisant partie du champ réel d'un instrument, que son image reçoive intégralement le flux incident. Il faut, plus encore, qu'clle posscde une netteté suffisante.

Nous considérons d'abord un système optique, - tel un objectif photographique on de lunctte, - donnant une image réelle. L'objet étant un plan normal à l'axe, son image, nous l'cxigeons, doit présenter le même caractère.

Le système (S) (fig. 2I), étant aplanétique, donnera en général une image $\mathrm{P}^{\prime}$ courbe, lieu des points géométriques images des points du plan $\mathbf{P}$. Aplanir $\mathbf{P}^{\prime}$ est une opération difficile qui complique, parfois outre mesure, le système $(\mathrm{S})$; on peut lui conserver une certaine courbure grâce à la tolérance de l'organe récepteur, - cil ou plaque. Considérons l'image physique trs 
d'un point quelconque de $\mathbf{P}$; ses bases envelopperont deux surfaces $\pi$ et $\pi^{\prime}$, coupant l'axe en $p$ et $p^{\prime}$, et com-

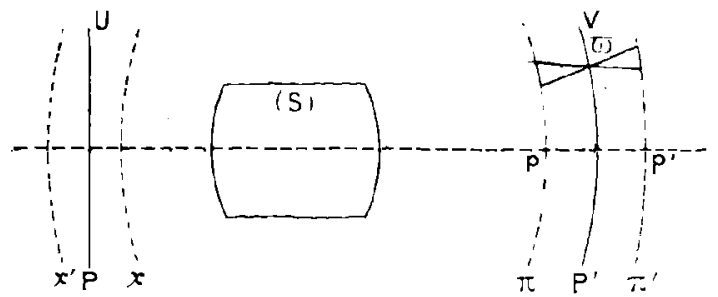

Fig. 21.

prenant entre elles un solide géométrique $\mathrm{V}$, dit volume de netteté. Un plan Q (fig. 23), situé entre $p$ et $p^{\prime}$, sera coupé par $\pi^{\prime}$ suivant un cercle contenu dans $\mathrm{V}$ et sur l'étendue duquel l'image aura toute la netteté désirable. Ce cercle déterminera, pour la mise au point $Q$, l'étendue du champ apparent, si d'ailleurs, ce que nous supposons, le système (S) transmet intégralement les faisceaux incidents correspondants. Ie champ, maximum quand $Q$ sera tangent $\dot{a} \pi$, deviendra nul lorsque le plan

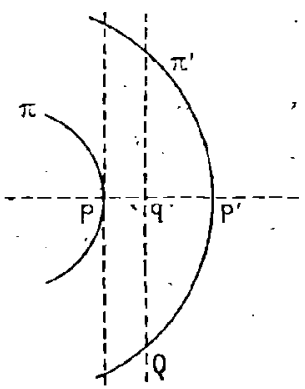

Fig. 22. passera par $p^{\prime}$; en $q$, il aura une valeur intermédiaire, mais avec cet avantage que celleci subsistera pour toute position de Q entre $p$ et $q$. II y aura donc, dans ce cas, une tolérance de mise au point. Cette tolérance est d'ailleurs indispensable; 
aussi doit-on arriver à une planéité suffisante de ces surfaces $\pi$ et $\pi^{\prime}$, presque parallèles à $\mathrm{P}^{\prime}$, de façon que la valeur prévue pour le champ reste acquise pour une variation convenable de mise au point.

Les aberrations ont pour effet de transformer l'image solide conique en image à cercle de gorge; sa longueur diminue, les surfaces $\pi$ et $\pi^{\prime}$ se rapprochent; mais les conclusions précédentes subsistent.

Quand on suppose le plan objet P à l'infini, le volume $\checkmark$ est dit alors volume focal, et son épaisscur $p p^{\prime}$ suivant l'axe est la profondeur de foyer.

99. Effet des diaphragmes de limitation sur l'amplitude du champ net. - L'épaisseur du volume de netteté est à peu près partout égale à la longueur de l'image solide. Cette dernière longueur, puisque les bases du solide sont de grandeur constante, dépend de l'aberration, diamètre du cercle de gorge, et de.l'ouverture angulaire de l'image, c'est-à-dire de celle du faisceau qui la forme. En fermant le diaphragme, on réduit l'ouverture du faisceau et aussi les aberrations. Tant que la diffraction reste inférieure à l'aberration, l'image solide s'allonge à la suite de la mancuvre du diaphragme, et, par suite, le champ s'agrandit ou la profondeur de foyer augmente. Ces conséquences sont bien connues des photographes.

Ioo. Profondeur de champ; profondeur de foyer. - L'image (fig. 2 I), à travers (S), du volume $V$, focal ou de nelteté, est un volume $U$ compris entre deux surfaces plus ou moins planes $\chi$ et $\chi^{\prime}$, situées de part et d'autre de P. Elles délimilent, pour la mise au point $\mathrm{P}^{\prime}$, le champ en profondeur. Leur intervalle $\mathrm{P}$, suivant l'axe dit profondeur de champ, est donné en 
fonction de la profondeur $p$ de foyer par les relations approximatives équivalentes :

$$
\mathrm{P}=\frac{p \mathrm{~F}^{\mathrm{z}}}{\left(\mathrm{D}^{\prime}-\mathrm{F}\right)-p^{\mathrm{2}}},
$$

$$
\mathrm{P}=\frac{p \mathrm{~F}^{2}(\mathrm{D}-\mathrm{F})}{\mathrm{F}^{2}-\frac{p^{2}}{4}(\mathrm{D}-\mathrm{F})},
$$

$\mathrm{D}$ et $\mathrm{D}^{\prime}$ étant les distances des plans $\mathrm{P}$ et $\mathrm{P}^{\prime}$ à leurs points nodaux respectifs, el $\mathrm{F}$ la focale du système. Quand $\mathrm{D}=\infty$, ou $\mathrm{D}^{\prime}=\mathrm{F}$, la formule est inapplicable. La profondeur de champ est alors infinie, et sa surface la plus voisine est à une distance $D_{0}$ du point nodal antérieur donnée par

$$
\mathrm{D}_{0}=\frac{\mathrm{F}(2 \mathrm{~F}-p)}{p}=\frac{2 \mathrm{~F}^{2}}{p} .
$$

Un objectif rigoureusement aplanétique, ouvert à $\frac{I}{j}$, possède une profondeur de foyer égale à

$$
p=0,0002 \mu \quad \text { ( } p \text { en mètres })
$$

en admeltant la nelteté à 0,1 millimètre.

Pour $\mu=10$ et $F=30$ centinètres, on a

$$
\mathrm{D}_{0}=900 \text { mètres. }
$$

Si le diamètre du cercle de gorge est de o,o r millimètre, on obtient aisćment :

$$
p=2(0,0001-0,00001) r_{1}=0,00018 i,
$$

el par suite dans les mêmes conditions :

$D_{0}=1$ ooo mètres.

ror. Champ net des lunettes. - Que les images soient réelles ou virtuelles, les considérations développées aux $\$ \$ 98$ à $\mathbf{\text { I }}$ oo s'appliquent à tous les systèmes optiques; mais elles sont plus délicates à mettre en 
ceuvre dans les lunettes qui rejettent l'image à l'infini, car ce dernier symbole est moins maniable en physique qu'en mathématique.

Répartissons en deux groupes les éléments optiques d'une lunette (fig. 23). L'un (S) comprendra, par excmple, l'objectif et, s'il y a lieu, quelques lentilles de l'oculaire; l'autre $\left(S^{\prime}\right)$, les verres restants. Le point $\Lambda$

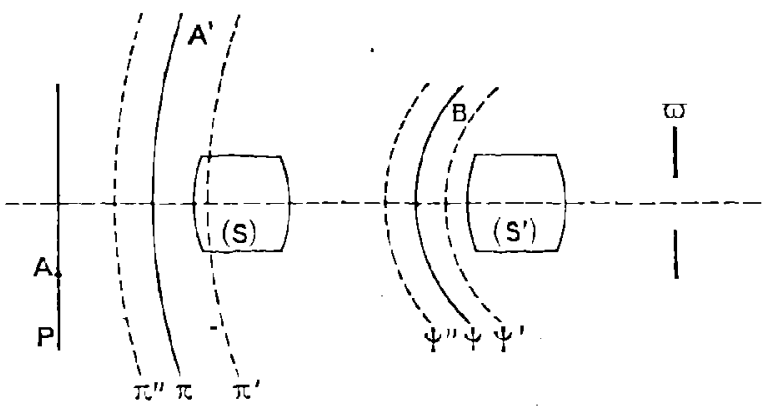

Fig. 23.

$\mathrm{du}$ plan objet $\mathrm{P}$ donne une image virtuelle en $\mathbf{A}^{\prime}$, image ponctuelle, en supposant le système total aplanétique. L'wil, reçoit le faisceau de sommet $A^{\prime}$ et s'appuyant sur la pupille apparente $\omega$.

L'cil, accommodé sur un plan ou une sphère telle que $\tau$, le sera également dans toute la région comprise entre les surfaces de mème nature $\pi^{\prime}$ et $\tau^{\prime \prime}$ qui limitent, en la circonstance, la profondeur du champ de l'oil. La position de $\mathrm{A}^{\prime}$ entre les plans limites sera donc indifférente.

Pour éliminer la considération du passage dircet à l'infini de ces divers éléments, formons les images $B$, 
$\psi, \bigcup^{\prime}, \psi^{\prime \prime}$ de $\mathbf{A}^{\prime}, \pi, \pi^{\prime}, \pi^{\prime \prime}$ à travers le système (S); et remarquons que $\mathrm{B}$ n'est autre que l'image de $\mathrm{A}$ à travers (S), ou plutòt le sommet de l'image physique $A^{\prime \prime}$.

Si cette image, lieu de $\mathrm{A}^{\prime \prime}$, était rigoureusement plane et de plus géométriquement ponctuelle (image physique de longueur nulle), les surfaces $\psi^{\prime}$ et $\psi^{\prime \prime}$ joueraient vis-a-vis de $\left(S^{\prime}\right)$, en ce qui concerne le champ possible de ce système partiel, le rôle des surfaces $\pi$ et $\pi^{\prime}$ du $s 8$.

$\mathrm{Si}$, le lieu du sommet de $\mathrm{A}^{\prime \prime}$ étant un plan, l'image physique a une longueur $\delta$, les surfaces $\psi^{\prime}$ et $\psi^{\prime \prime}$ devront être remplacées par des surfaces, extérieures et sensiblement parallèles à elles, à une distance $\delta$.

Enfin, quand le lieu de $\mathbf{A}^{\prime \prime}$ sera une surface courbc, c'est cette dernière qui remplacera, avec ses variations possibles de forme, le plan de mise au point considéré au $\$ 98$ précité.

Lorsque l'oeil est accommodé pour l'infini, vision normale, $\pi$ s'y transporte, et son image $\dot{y}$ devient la surface focale de $\left(S^{\prime}\right)$; on peut alors faire coïncider $\pi^{\prime \prime}$ et $\pi$, et par suite $\psi^{\prime \prime}$ et $\psi$, si l'on ne tient pas à user de la faculté que possède l'oeil d'utiliser des faisceaux légè rement divergents: $\pi$ 'se dispose à 25 mètres environ de l'œil, et son image $\varphi$ ' devient, par définition, la suifface hyperfocale de $\left(\mathrm{S}^{\prime}\right)$.

ro2. - L'œil est supposé, jusqu'ici, conserver son accommodation. La souplesse de cette dernière permet d'espacer encore les surfaces $\pi$ et $\pi$ ', et par suite $\psi$ et $\psi$ ', eu prenant pour $\pi^{\prime}$ celle qui répond à la distance minimum de la vision distincte, 250 millimètres environ. C'est souvent à la surface $\psi^{\prime}$ correspondante qu'on réserve le qualificatif d'hyperfocale. Lorsqu'on 
utilise tout ce volume focal du système ( $\left.\mathrm{S}^{\prime}\right)$, ure certaine région du champ est vue distinctement par l'œil au repos; dans l'autre partie, l'organe doit successivement s'accommoder afin que la vision soit nette. Il s'ensuit une certaine gêne, qu'il est utile d'éviter quand on le peut.

Io3. - Les considérations développées ci-dessus ( $\mathrm{I} O \mathrm{r}-\mathrm{IO}_{2}$ ) sont générales; elles ne visent aucun groupement spécial de verres. En pratique, il est avantageux de constituer les systèmes $(S)$ et $\left(S^{\prime}\right)$ de manière que le premier donne des images réelles, et, plus particulièr'ement, de le réduire à l'objectif. Dans ce dernier cas, (S') étant l'oculaire, les surfaces focale et hyperfocale s'y rapportent, et le volume compris entre eux est le volume focal de l'oculaire; il est en relation étroite avec le champ apparent, dont la grandeur caractérise la qualité de cette partie de l'instrument.

ro4. Mise au point variable et profondeur du champ. - Les deux surfaces $\pi$ et $\tau^{\prime}$, qui, dans un système quelconque, limitent le volume de netteté, permettent de placer le plan $\mathrm{P}^{\prime}$ de mise au point dont l'aire de netteté est maximum. Il n'y a pas lieu de chercher autre chose quand l'image doit être recue par une plaque sensible; mais si elle doit être observée par l'oeil, il peut y avoir intérêt, après avoir examiné l'image nette formée sur $P^{\prime}$, à déplacer la mise au point en $\mathrm{P}^{\prime \prime}$, par exemple ${ }^{1}$, pour explorer la région $m n$ $m^{\prime} n^{\prime}$,qui deyient nette. Le champ total, limité par mn,

1 Se reporter a la figure 22, -. Marquer sur cette figure un point $\mathbf{P}^{\prime \prime}$, à gauche de $\mathrm{P}^{\prime}$, et mener, perpendiculairement à $\mathbf{P P}^{\prime}$, une droite coupant les deux courbes $\pi$ et $\pi^{\prime}$ respectivernent en $m, m^{\prime}$ et en $n, n^{\prime}$. 
s'élargit dans cette opération; mais la netteté n'apparaissant que successivement, suivant la région de l'image, on dit que le champ est courbe.

Dans les appareils, où l'un des systèmes partiels est mobile par rapport à l'autre (oculaire des lunettes se déplaçant par rapport à l'objectif, ou verre d'œil par rapport au restant de l'oculairc), cette variation de mise au point est obtenue, sans différence d'accommodation de l'oil, par le déplacement relatif des systèmes; alors (fig. 23 ), si l'image due à (S) est nette sur une étendue assez grande, ses différentes régions peuvent entrer dans le volume de netleté de (S'), que ce dernier système entraîne avec lui. La courbure du champ peut tenir à l'un ou à l'autre des systèmes. Si (S) est un objectif de lunette ou de microscope, la courbure du champ, quand elle existe, doit être généralement attribuée à l'oculaire. 


\section{GHAPITRE VI}

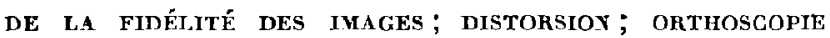

ro5. Cause de la distorsion. - L'image d'un objet plan, normal à l'axe d'un système, est, nous le supposons, dans un autre plan parallèle au premier. Le système qui la fournit est achromatique. Dans ces conditions, la fidélité de l'image n'exige que sa similitude géométrique avec l'objet.

Quand cette propriété fait défaut, on dit que l'image présente de la distorsion.

La distorsion est une conséquence immédiate des aberrations de sphéricité.

Soit $\mathbf{D}$ le diaphragme matériel efficace de limitation (supposé réduit à un plan géométriquc) pour un système optique (fig. 24) composé de deux systèmes particls $\left(\mathrm{S}^{\prime}\right)$ et $\left(\mathrm{S}^{\prime \prime}\right)$, dont l'un pourra d'ailleurs disparaitre sans que la théorie soit modifiée.

Un faisceau réel, $\rho$, limité effectivement par $\mathbf{D}$, sera virtuellement limité à son entrée par l'ouverture apparente $\mathrm{D}^{\prime}$ et à sa sortie par l'ouverture apparente $\mathrm{D}^{\prime \prime}$, images respectives de $\mathrm{D} \dot{\mathrm{a}}$ travers $\left(\mathbf{S}^{\prime}\right)$ et $\left(\mathbf{S}^{\prime \prime}\right)$. Mais, si 
DE LA FIDÊLITÉ DES IMAGES. DISTORSION. ORTHOSCOPIE 127 D est un plan géométrique, il n'en est pas de même de $\mathrm{D}^{\prime}$ et de $\mathrm{D}^{\prime \prime}$.

L'ouverture $\mathbf{D}^{\prime}$, par exemple, est, pour le faisceau $\rho$, le lieu des images $a^{\prime}$ des points a d'intersection du faisceau par le plan D; l'image $a^{\prime}$ étant formée par le rayon, le pinceau qui, dans le faisceau o, passe par le point $a$.

Dans ces conditions, $\mathrm{D}^{\prime}$ pourrait être courbe; mais

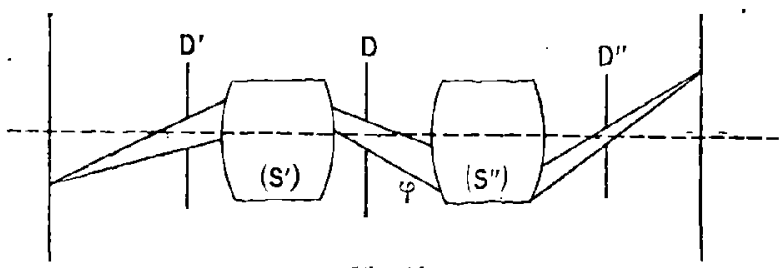

Fig. 24.

elle est une surface géométrique. Nous la supposerons plane, admettant pour les systemes partiels la planéité accordée au système total.

Quand on envisagera un faisceau autre que $\wp$, l'image $\mathrm{D}^{\prime}$, lieu des mêmes points $a$, se déplacera, à cause des aberrations, parce que le pinceau qui formera l'image de $a$, changeant d'inclinaison, tombera sur des régions différentes des surfaces du système $\left(\mathbf{S}^{\prime}\right)$.

L'image - sans autre spécification - de D par rapport à (S) est cet ensemble continu de plans, et par suite un petit solide cylindrique dont la hauteur, dirigée suivant l'axe, est finie : son existence est la conséquence de l'aberration de sphéricité.

Une autre conséquence de cette aherration est la suivante : soient $\alpha, \alpha^{\prime}, \alpha^{\prime \prime}$ les angles respectifs, avec l'axe, 
des pinceaux centraux respectifs des faisceaux réfracté $\emptyset$, incident et émergent. En général, ni $\operatorname{tg} \alpha^{\prime}$, ni $\operatorname{tg} \alpha^{\prime \prime}$ ne sont proportionncls à $\operatorname{tg} \alpha$. Il s'ensuit que le rapport de $\operatorname{tg} \alpha^{\prime \prime}$ à $\operatorname{tg} \alpha^{\prime}$ est une fonction $u$ de l'angle $\alpha$.

Cela constaté, la fig. 25 , qui est la reproduction géo-

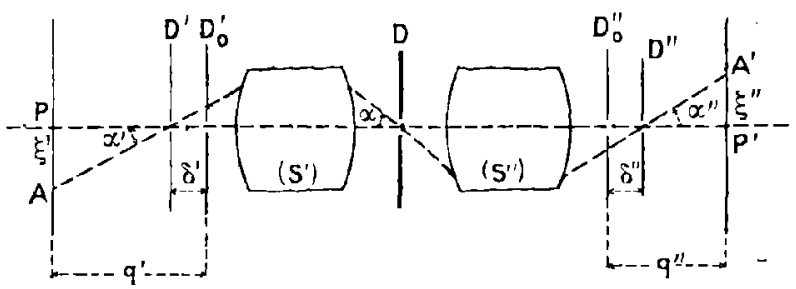

Fig. 25.

métrique de la précédente, donne aisément la relation :

$$
\xi^{\prime \prime}=\xi^{\prime} \frac{u\left(q^{\prime \prime}-\delta^{\prime \prime}\right)}{q^{\prime}-\delta^{\prime}}
$$

La proportionnalité de $\xi^{\prime \prime}$ à $\xi^{\prime}$, qui est la condition de l'orthoscopie, exigerait donc que les trois fonctions $u$, $\delta^{\prime}$ et $\delta^{\prime \prime}$ de l'angle $\alpha$ fussent liées entre elles par une relation telle que :

$$
k\left(q^{\prime}-\delta^{\prime}\right)=u\left(q^{\prime \prime}-\delta^{\prime \prime}\right)
$$

$k$ étant une cortaine constante, qui n'est autre que le grossissement linéaire axial.

ro6. Distorsion nodale. - On peut, dans l'étude de la distorsion, substituer au grossissement central et aux ouvertures apparentes tels autres éléments géométriques qui parâtront, suivant les circonstances, plus accessibles. En particulier, la considération des points nodaux offre un certain intérêt. 
DE LA FIDËLITÉ DES IMAGES. DISTORSION. ORTHOSCOPIE 129

Supposons que, le diaphragme D s'ouvrant suffisamment, l'image $\boldsymbol{\Lambda}^{\prime}$ du point $\boldsymbol{A}$ demeure un point physique. Il y aura alors deux rayons conjugués, un dans chaque faisceau (incident et émergent), et parallèles, qui couperont l'axe aux points nodaux $N^{\prime}$ et $\mathrm{N}^{\prime \prime}$ répondant à l'angle $\alpha$. A cause des aberrations, ces points seront distincts des nœuds centraux $\mathrm{N}_{0}^{\prime}$ et $\mathrm{N}_{0}^{\prime \prime}$ que détermine la valeur nulle de $\alpha$.

La géométrie donne alors sur la fig. 26 :

$$
\xi^{\prime \prime}=\xi^{\prime} \frac{s^{\prime \prime}+\varepsilon^{\prime \prime}}{s^{\prime}+\varepsilon^{\prime}} \text {. }
$$

La variation de position des ouvertures apparentes n'intervient plus, ce qu'explique l'élargissement des

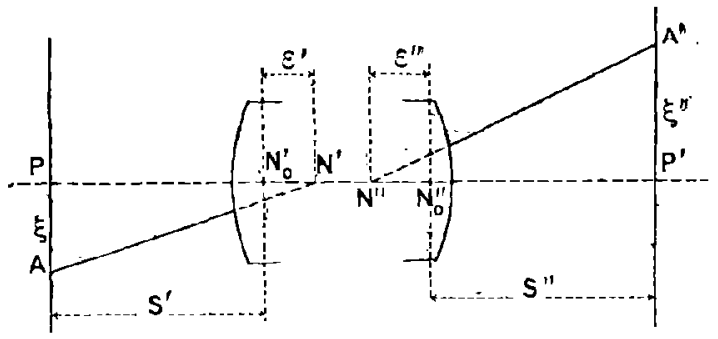

Fig. 26.

faisceaux. La cause directe de la distorsion paraît être la variation de position des points nodaux avec l'incidence des faisceaux. On a donné à ce mode de déformation des images le nom de distorsion nodale. Les hy pothèses qui la concernent sont assez bien réalisées dans les objectifs de lunettes et les objectifs photographiques doubles travaillant à grande ouverture. 
107. Distorsion locale. - Si l'un considère (fig. 27) une lentille infi-

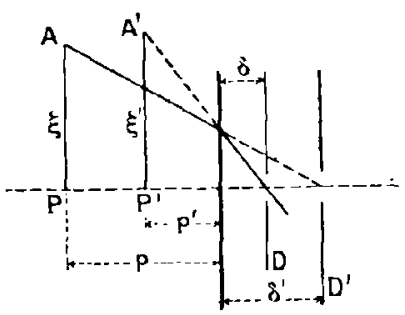

Fig. 27. niment mince élroitement diaphragmée en D, le faisceau incident, émané du point objet $\mathrm{A}$, qui donne le faisceau émergent utile passant en $\mathrm{D}$, a pour axe la droite joignant le point A au centre de l'imáge $\mathrm{D}^{\prime}$ de $\mathbf{D}$ par rapport à la lentille. On a ici :

ou

$$
\begin{aligned}
& \xi^{\prime}=\xi \frac{\Sigma^{\prime}\left(p^{\prime}+\delta\right)}{\partial\left(p+\partial^{\prime}\right)}, \\
& \xi^{\prime}=\xi \frac{\partial\left(p^{\prime}+\delta^{\prime}\right)}{\partial^{\prime}\left(p+\partial^{\prime}\right)},
\end{aligned}
$$

suivant que le diaphragme est en arrièré ou en avant de la lentille.

à étant variable, $亏^{\prime}$ n'est pas proportionnel à $\xi$; l'image présente donc de la distorsion. Caractérisée par la seule grandeur $\partial^{\prime}$, et par suite par la hauteur $h$ du point sur lequel tombe le rayon axial du faisceau incident, on peut donner à cette déformation le nom de distorsion locale. C'est elle qui entre en jeu dans les diverses lentilles des oculaires, dans les objectifs photographiques simples.

\section{Formes des images distordues. - Les} considérations qui conduisent aux notions de distorsion nodale et de distorsion locale ne sont pas suffisamment précises pour qu'il y ait intérêt à utiliser les consé quences éloignées des formules qui leur correspondent. 
DE LA FIDÉLITB́ DES MARES. DISTORSION. ORTHOSCOPIE 131 La forme simple de ces dernières peut cependant rendre d'utiles services dans l'explication qualitative des phénomènes.

La relation générale et rigoureuse établie plus haut (ro5) doit en général leur être préférée; elle est parfois aussi maniable. Nous l'utiliserons d'abord pour la distinction des deux formes types des images distordues.

En se reportant à la figure et aux notations du $\$$ ro5, considérons (fig. 28) une droite oljet (M) perpendiculaire au plan du tableau et à l'axe du systeme, et à une distance $h$ de celui - ci.

L'image de (M) à travers (S) est le lieu $\left(\mathrm{M}^{\prime}\right)$ des points $\mathbf{A}^{\prime}$, images des divers points $\mathbf{A}$ de la

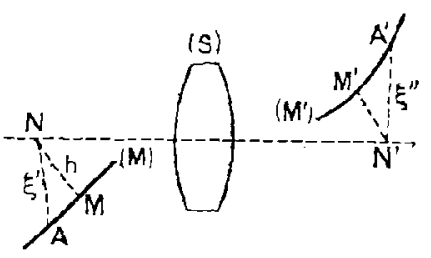

Fig. 28. droite; elle est dans un plan normal à l'axe en $\mathbf{N}^{\prime}$, imagge de $\mathbf{N}$. On a, pour déterminer les points $\mathrm{A}^{\prime}$, la relation :

$$
\check{\xi}^{\prime \prime}=\xi^{\prime} \frac{u\left(q^{\prime \prime}-\partial^{\prime \prime}\right)}{q^{\prime}-\partial^{\prime}}
$$

D'ailleurs $\xi^{\prime}, \xi^{\prime \prime}$ et l'axe sont dans un mème plan. Si le coefficient de $\xi^{\prime}$ dans la relation précédente était

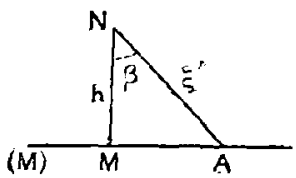

Fig. 29,

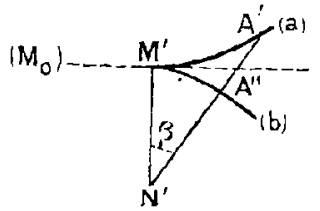

$\mathbf{N}^{\prime}$

une constante, les images $\mathbf{A}^{\prime \prime}$ de $\mathbf{A}$ (fig. 29) seraient 
sur une droite $\left(\mathrm{M}_{0}\right)$ perpendiculaire à $\mathrm{M}^{\prime} \mathrm{N}^{\prime}$. Mais ce coefficient est; on l'a remarqué, une fonction de $\alpha$, et on peut écrire la relation précédente :

$$
\xi^{\prime \prime}=\xi^{\prime} \varphi(x) \text {. }
$$

On tire aisément de cette relation, en désignant $\mathbf{N}^{\prime} \mathbf{A}^{\prime \prime}$ par $\xi_{0}^{\prime \prime}$ :

$$
\xi^{\prime \prime}-\xi_{0}^{\prime \prime}=\xi_{a}^{\prime \prime}\left[\varphi(\alpha)-\rho\left(\alpha_{0}\right)\right] .
$$

L'angle $\alpha$ varie avec $\beta$, et croît généralement avec lui. Si $\varphi(\alpha)>\varphi\left(\alpha_{0}\right), \xi^{\prime \prime}-\xi_{0}^{\prime \prime}>\xi_{0}^{\prime \prime}$, c'est-à-dire que $\mathbf{A}^{\prime \prime} \mathbf{A}^{\prime}$ scra positif, et $\Lambda^{\prime}$ se tiendra toujours au-dessus de la droile $\mathbf{M}^{\prime} \mathrm{A}^{\prime \prime}$, et on aura, comme figure de $\mathbf{M} \Lambda$, l'apparence $(a)$; si, au contraire, $\phi(\alpha)<\varphi\left(\alpha_{0}\right)$, on obtiendra l'apparence $(b)$.

En considérant (fig. 3o) un carré centré $(c)$ sur l'axe, son image aura,

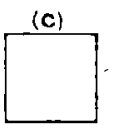

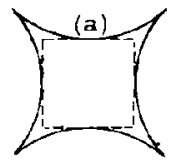

Fig. 30.

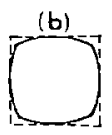
suivant les circonstances, la forme $(a)$ ou la forme $(b)$.

Dans le premier cas, on dit que la distorsion est en croissant; dans le second, qu'elle est en barillet ou en tonneau. Si, au lieu d'un carré, on considère des cercles, à l'équidistance $\varepsilon$, centrés sur l'axe, la distance $\varepsilon$ ' entre les images de deux cercles de rayons $p$ et $p+\varepsilon$ aura pour expression :

$$
\varepsilon^{\prime}=s p\left(x_{0}\right)+(p+\varepsilon)\left[p(\alpha)-\varphi\left(\alpha_{0}\right)\right] .
$$

L'intervalle des cercles images ira donc en augmentant, à mesure qu'on s'éloignera du centre du champ, Jorsque la distorsion sera en croissant; elle diminuera dans la distorsion en barillet. 
DE LA FIDÉlité DES IMAGES. DISTORSION. ORTHOSCOPIE 133

109. Distorsion dans les lunettes et les microscopes. - Dans les lunettes observant à grande distance et rejetant au loin l'image, la formule génćrale du $\$$ rō̃ se simplifie; $\chi^{\prime}$ et $\AA^{\prime \prime}$ ont en général des valeurs finies et très petites. La relation en question, puisque $q^{\prime}$ et $q^{\prime \prime}$ sont infinis, se réduit alors à :

$$
\xi^{\prime \prime}=u \xi^{\prime}
$$

le coefficient $u$ de distorsion n'est autre que le grossissement linéaire.

En passant au grossissement angulaire $\mathrm{G}$, on a :

$$
u=\mathrm{GG}_{0}^{-2} \text {, }
$$

$\mathrm{G}_{0}$ étant le grossissement angulaire central. La constance de $u$, caractère de l'absence de distorsion, entraîne l'invariabilité du grossissement G.

Dans les microscopes, si leur construction annule $\varepsilon^{\prime}$ et $\varepsilon^{\prime \prime}$, la disparition de la distorsion est liée aussi à la constance du grossissement angulairc ou linéaire. Mais, comme l'image se forme à distance finie, il est indispensable, pour qu'elle paraisse sans déformation, que l'œil se dispose au cercle oculaire C (fig. 3I); sinon, l'œil, étant par excmple en arrière, voit un point image $\mathrm{A}^{\prime}$ sous l'angle $v^{\prime}$, différant de $v$, et c'est celui-ci seulement qui est déterminé en fonc-

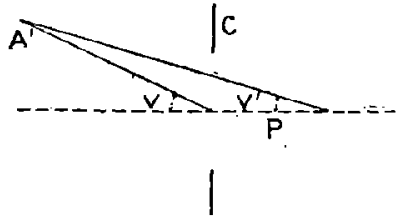

Fig. 31. tion de l'angle correspondant au point objet, de façon à assurer la constance du grossissement. C'est pour cette raison que le centre de l'anneau oculaire a reçu le nom de point orthoscopique. 
I ro. Correction de la distorsion. - La correc tion de la distorsion exige que l'on ait identiquement :

$$
u_{0}\left(q^{\prime}-\delta^{\prime}\right)-u\left(q^{\prime \prime}-\delta^{\prime \prime}\right)=0, .
$$

quel que soit $\alpha, u_{0}$ étant le grossissement linéaire eentral.

Il est, en général, impossible de satisfaire rigoureusement à cette identité. On se contente le plus souvent d'assurer l'égalité à zéro du premier membre de cette équation pour une, ou quelquefois deux valeurs de $\alpha$, convenablement choisies dans le champ. C'est dans ce sens qu'on dit que la distorsion est annulée pour ces valeurs du champ : le grossissement linéaire $\mathrm{y}$ est alors égal au grossissement central. Si, en outre, on a pu réaliser, dans ces cas, la nullité de $\delta^{\prime \prime}$, le centre de la surface de sortie est point orthoscopique pour les mêmes valeurs du champ.

Les notions relativement simples de distorsion nodale de distorsion locale peuvent être utilisées dans une première approximation.

Toutes choses égales d'ailleurs, la distorsion change de forme avec le sens de déplacement des points nodaux par rapport aux noeuds centraux : les formules établies plus haut le montrent sans difficulté. En mettant l'un à la suite de l'autre deux systèmes identiques ou à peu près, mais symétriques par rapport à un plan normal à leur axe, la distorsion produite par l'ensemble sera, sinon annulée, du moins sensiblement réduite. C'est l'une des raisons de succès des objectifs symétriques doubles.

Quant à la distorsion locale, elle change de sens quand le diaphragme se porte d'un côté ou de l'autre 
DE LA FIDÉLITÉ DES IMAGES. DISTORSION. ORTHOSCOPIE 135 de la lentille, quand l'objet, de réel, devient virtuel, quand la lentille convergente est remplacée par un élément divergent. Ces simples remarques permettront, non de calculer, mais de se faire une idée de la façon dont on pourra réduire simplement la distorsion. L'une d'elles est utilisée dans l'objectif symétrique double cité ci-dessus : le diaphragome, postérieur pour la combinaison d'avant, est antérieur à l'autre.

On pourra, à l'aide des remarques précédentes, se rendre compte de la forme de la distorsion, en observant que la loupe (lentille convergentc) donne à l'oil (pupille-diaphragme en arrière) une image distordue en croissant d'un objet réel.

\section{I. Distorsion et grossissement dans la loupe.} -- La distorsion est à éviter dans les instruments optiques. Elle est pourtant utilisée, involontairement, dans la loupe à lire.

La Physique élémentaire apprend que le grossissement d'une loupe est maximum quand l'oeil y est applicpué le plus près possible. La pratique montre, sans contcstation aucune, le contraire : lorsqu'on lit à la loupe, les caractères paraissent d'autant plus grands que l'œil est plus éloigné de l'instrument. La distorsion loçale en donne une explication suffisante, à condition de ne pas attribuer une trop grande importance aux formules.

La loupe $L$ (fog. 3a) donnera à l'oil dont la pupille est en $P$ une imagre virtuelle circulaire d'une petite circonférence, de rayon $\rho$, lelle que $a b$. Le faisceau incident passera par l'image $\mathrm{P}^{\prime}$ de $\mathrm{P}$ répondant à l'inclinaison de ce faisceau.

Désignons par $\varepsilon$ l'aberration longitudinale de $\mathrm{P}^{\prime}$ : elle place ce point entre la lentille et le foyer conjugué principal de P. En supposant, pour simplifier les calculs, que $a b$ se trouve dans le plan focal de la loupe, la figure et l'équa- 
tion des foyers conjugués centraux des lentilles minces conduisent à la relation :

$$
\operatorname{tg} \alpha=\frac{\hat{\rho}}{p} \frac{p f-\varepsilon(p-f)}{f^{2}-\varepsilon(p-f)} .
$$

Si $p=0$, on sait que :

$$
\operatorname{tg} \alpha_{0}=\frac{p}{f} \text {. }
$$

Si $p=m f$, avec $m>\mathbf{I}$, la formule donne :

$$
\operatorname{tg} \alpha=\frac{m f-(m-I) \varepsilon}{m \tilde{f}-m(m-I) \varepsilon} \operatorname{tg} \alpha_{0} .
$$

$\alpha$ est donc toujours supérieur à $\alpha_{0}$, d'aulant plus que $m$ est

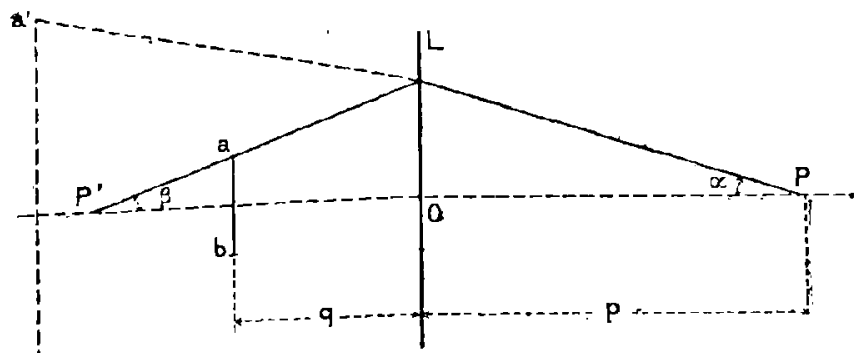

Fig. 32 .

plus grand, c'est-à-dire que l'ocil est plus loin de la lentille, - au delà d'ailleurs du foyer principal.

La distorsion se manifeste d'ailleurs parce que $\varepsilon$ dépend de $\alpha$, par suite de $\rho$.

Ajoutons que c'est aux dépens de la netteté qu'on obtient le grossissement en question; mais tant qu'elle reste suffisante pour distinguer aisément les caraclères d'imprimerie, il peut y avoir avantage à sacrifier la finesse des images. Il en serait autrement de la loupe de l'horloger : c'est à elle que s'applique la théorie classique. 


\section{CHAPITRE VII}

DES IMAGES DANS LES IYSTRUMEYTS BLYOCUIARES

II2. - La vision simultanée par les deux yeux est aidée, on le sait, par l'emploi de systèmes optiques spécialement appropriés. Pour l'observation à grande distance, les instruments en question, dénommés jumelles, sont constitués par deux lunettes domnant des images droites (longues-vues terrestres ou lunettes de Galilée), placées parallèlement et à l'écartement des yeux de l'observateur.

Nous supposerons ici les deux systèmes partiels identiques, et chacun d'eux réduit à deux lentilles infiniment minces, l'objectif $O$ et l'oculaire $\omega$; on admettra en outre la coïncidence des centres optiques de l'œil et de l'oculaire correspondant. Cette organisation schématique simplifiera l'étude des instruments, et les formules en cause resteront suffisamment exactes pour les conclusions, d'ordre surtout qualitatif, que nous en tirerons. Les principes évoqués permettraient d'ailleurs, le cas échéant, de faire des calculs plus exacts, tout aussi élémentaires, mais plus prolixes.

Ix3. Superposition thérique des images. 
- Les axes des deux lunettes constituant la jumelle seront d'abord supposés parallèles.

Un point $M$ (fig. 33) du plan des axes donne les deux images $\mathrm{N}$ et $\mathrm{N}$, une

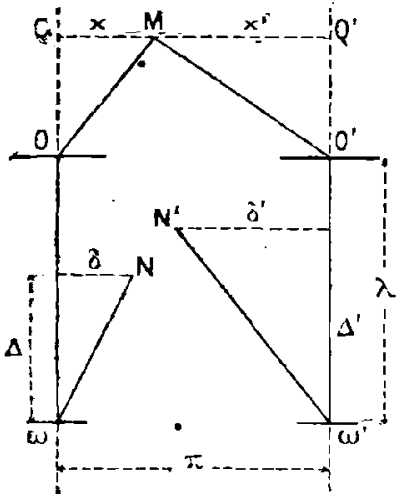

Fig. 33. dans chaque système partiel. Leurs positions sont données par les relations aisées à établir géométriquement :

$$
\begin{aligned}
& \S=x \frac{\mathrm{F}}{f} \frac{\Delta+f}{\mathrm{D}-\mathrm{F}}, \\
& \varepsilon^{\prime}=x^{\prime} \frac{\mathrm{F}}{f} \frac{\Delta^{\prime}+f}{\mathrm{D}-\mathrm{F}},
\end{aligned}
$$

avec $x+x^{\prime}=\sigma$.

Quant a $\Delta$ et $\Delta^{\prime}$, elles sont déterminées par les valenrs $\lambda$ et $\lambda^{\prime}$ des longueurs des lunettes, distances que l'observateur a cru devoir mettre entre les objectifs et les oculaires correspondants :

$$
\lambda=\frac{\mathrm{F}+f+\mathrm{F} f\left(\frac{\mathrm{I}}{\Delta}-\frac{\mathrm{I}}{\mathrm{D}}\right)}{(\mathrm{I}-\mathrm{F})\left(\mathrm{I}+\frac{f}{\Delta}\right)}=\mathrm{DF}-\mathrm{D}-\mathrm{F}+{ }_{\Delta+f}+
$$

$\lambda^{\prime}$ s'en déduit en remplaçant, dans le second membre de la relation, $\Delta$ par $\Delta^{\prime}$.

Ces relations supposent l'objectif et I'oculaire, de focales respectives $\mathrm{F}$ et $f$, tous deux convergents. Quand (lunette de Galilée) l'oculaire est divergent, on doit attribuer à $f$ un signe négatif dans la dernière 


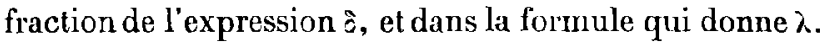

Remarquons en outre que, la lunette ne renversant pas les images, $\omega N$ est du même côté de l'axe que OM, ou, en d'autres 'termes, que $x$ et ò ont même signe (l'origine de ces abscisses étant sur l'axe $\omega O$ ).

S'il était nécessaire que la vision de l'image s'effectuât dans les mêmes conditions que celle d'un objet, la coïncidence des points $\mathrm{N}$ et $\mathrm{N}^{\prime}$ devrait être assurée. On aurail donc par suite :

$$
\Delta=\Delta^{\prime}=\frac{\mathrm{D} f}{\mathbf{F}}-2 f \text {. }
$$

En appelant G le grossissement théorique de la lunette, donné par $\mathrm{F}=\mathrm{G} f$, et négligeant of dans le second membre de l'équation précédente, celle-ci devient :

$$
\Delta=\Delta^{\prime}=\frac{\mathrm{D}}{\mathrm{G}} \text {. }
$$

Cette relation est d'ailleurs rigoureuse, le calcul le prouve, pour la lunette de Galilée.

La longueur correspondante commune aux deux corps de la jumelle est donnée par la formule :

$$
\lambda=\mathrm{D}\left(\frac{\mathrm{F}}{\mathrm{D}-\mathrm{F}}+\frac{f}{\mathrm{D}+\mathrm{F}}\right) .
$$

La condition $(a)$ étant indépendante de $x$, la coïncidence assurée pour les images du point M l'est aussi pour celles des points de la droite QQ'; un calcul élémentaire montrerait qu'il en est de mème pour tous les points du plan passant par M. et normal aux axes de l'instrument.

Done, dans la vision binoculaire à l'aide d'un instrument de grossissement théorique $G$, tout se passe 
comme si l'objet était ramené à une distance $G$ fois plus faible et observé alors directement.

\section{Ir4. Superposition physique des images.} - La théorie de la vision binoculaire instrumentale ne saurait s'arrêter à cette constatation, car elle supposerait une précision infinie dans le réglage des longueurs des lunettes. Grâce aux propriétés de l'œil, la superposition des images se maintiendra sans que la coïncidence des points correspondants soit réalisée. Et d'abord, la pereeption visuelle resterait unique, si la coïncidence des points $N$ et $\mathbf{N}^{\prime}$, au lieu d'être assurée géométriquement, ne l'élait que physiquement, c'est-àdire si les angles $N \omega N^{\prime}$ et $N_{\omega} \omega^{\prime} N^{\prime}$ étaient tous deux inférieurs à l'acuité visuclle ( $\mathrm{I}^{\prime}$ environ). Cette condition, soumise au calcul, exige, dans les cas usuels, un réglage, à quelques microns près, de la différence des longueurs des lunettes.

On pourrait encore demander que l'un des systèmes partiels étant réglé à la longueur $\lambda$, l'autre amenât l'image $N$ ' dans le champ de profondeur de l'œil mis

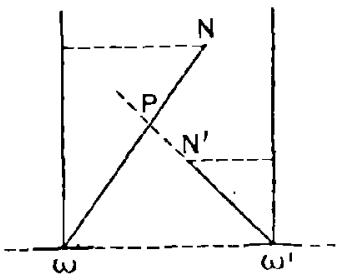

Fig. 34. au point sur $\mathrm{N}$, et à une distance augulaire de ce dernier point au plus égale à une minute. Dans ces conditions, $\lambda^{\prime}$ devrait être égal à $\lambda$, à quelques centièmes de millimètre près.

Ces exigences sont inutiles. L'coil placé en $\omega$ ne voit que l'image $\mathbf{N}$, l'œil situé en $\omega^{\prime}$ n'aperçoit. que $N^{\prime \prime}$. Les lignes visuelles convergeront vers $\mathrm{P}$ (fig. 34) à l'intersection de $\omega \mathrm{N}$ et de $\omega{ }^{\prime} \mathrm{N}^{\prime}$. 
IDS IMAGES DAYS LES IYSTRLHENTS RIYOCULAIRES $1 \% 1$

C'est en ce point qu'apparaîtra l'image unique de $\mathrm{M}$, si toutefois chaque image $\mathrm{N}$ ou $\mathrm{N}^{\prime}$ est dans le champ de profondeur de l'oeil mis au point en $\mathrm{P}$.

La différence maximum des longueurs des deux lunettes est :

$$
\begin{gathered}
\mathbf{L}=\lambda-\lambda^{\prime}=f\left(\frac{\Delta}{\Delta+f}-\frac{\Delta^{\prime}}{\Delta^{\prime}+f}\right) \\
=f^{2} \frac{\Delta-\Delta^{\prime}}{(\Delta+f)\left(\Delta^{\prime}+f^{\prime}\right)},
\end{gathered}
$$

$\Delta$ correspondant à la limite éloignée du champ de profondeur sur $\omega \mathrm{P}, \Delta^{\prime}$ à la limite rapprochée sur $\omega^{\prime} \mathrm{P}$.

On pourrait exprimer $\Delta$ et $\Delta^{\prime}$ en fonction de la distance $\Delta_{0}$ de $\mathrm{P}$ à $\omega \omega^{\prime}$. Les équations exactes sont, même dans l'hy pothèse où nous nous sommes placé, pénibles à discuter; la précédente nous suffira si le champ est assez faible pour que l'on puisse confondre les lignes visuelles avec leurs projections sur les axes.

En traduisant algébriquement Ia règle indiquée (36) relative à la profondeur de la vision, on a :

$$
\Delta=\Delta_{0}+\frac{\Delta_{0}^{2}}{15-\Delta_{0}}, \quad \cdot \Delta^{\prime}=\Delta_{0}-\frac{\Delta_{0}^{2}}{15+\Delta_{0}},
$$

l'unité de longueur étant le mètre,

ou

$$
\Delta=\frac{\mathrm{I} 5 \Delta_{0}}{\mathrm{I} 5-\Delta_{0}}, \quad \Delta^{\prime}=\frac{15 \Delta_{0}}{\mathrm{I} 5+\Delta_{0}} .
$$

On remarquera que si, au lieu d'images, on avait à considérer des objets, la valeur de $\Delta$ ne pourrait être négative. Pour $\Delta_{0} \supseteq 15$, on devrait faire $\Delta=\infty$.

Dès que l'on peut négliger la focale $f$ de l'oculaire devant la distance de mise au point $\Delta_{0}$, au moins égale 
à 25 centimètres, les relations ci-dessus conduisent à la valeur simple :

$$
\mathrm{L}=-\frac{x}{15} f^{2} . \quad \text { (unité : mètre) }
$$

En prenant un cas très courant d'une jumelle pour laquelle $G=8$ ct $f=20$ millimètres, on a :

$$
I_{L}=0^{\mathrm{mm}}, 05 \text { environ. }
$$

\section{ir5. Fusion physiologique des images. -} Un calcul plus exact ne modifierait pas l'ordre de grandeur de la différence $L$. Une pareille précision de réglage ne peut être atteinte que par un observateur exercé et dans des conditions de vision excellentes. Dans la pratique courante, il est difficile d'admettre que les images ne sortent pas des champs de profondeur respectifs des yeux qui les observent. La perception est cependant unique. C'est que chaque oil s'accommode à la distance particulière de l'image qu'il discerne; l'insensibilité de la vision monoculaire en ce qui regarde la profondeur permet ensuite de placer la double image au point unique d'intersection des deux lignes visuelles. Si une telle accommodation, différente pour, l'un et l'autre oil, et, par suite, si peu naturelle, peut être effectuée, le fait tient à l'absence, dans le champ de vision, de point de repère réel qui puisse faire juger de la non-coüncidence de chacune des images avec la rencontre des lignes visuelles.

Cette anomalie d'accommodation se traduit d'ailleurs par une fatigue des organes de la vue, fatigue bien connue de tous ceux qui, sans précautions, se servent d'une jumelle : chaque wil est accommodé pour une 
distance différente, dont l'une au moins ne correspond pas au degré de convergence des organes.

Cette convergence est, elle, presque invariable, quels que soient les réglages individuels des deux lunettes, Sa valeur est, en effet,

$$
\begin{gathered}
\beta=\operatorname{arctg} \frac{\partial}{\Delta}+\operatorname{arctg} \frac{\partial^{\prime}}{\Delta^{\prime}} \\
=\operatorname{arctg} \frac{\mathrm{G}}{\mathrm{D}-\mathrm{F}} \frac{\omega+f\left[\frac{x}{\Delta}-\frac{x}{\Delta^{\prime}}+\frac{\sigma}{\Delta^{\prime}}\right]}{\mathrm{I}-\mathrm{G}^{2} \frac{x}{\mathrm{D}-\mathrm{F}} \frac{x^{\prime}}{\mathrm{D}-\mathrm{F}}\left(\mathrm{I}+\frac{f}{\Delta}\right)\left(\mathrm{I}+\frac{f}{\Delta^{\prime}}\right)} ;
\end{gathered}
$$

elle est très voisine de

$$
\operatorname{arctg} \frac{G \pi}{D}
$$

dans la plupart des instruments : l'image unique binoculaire reste donc, comme dans le cas du réglage parfait, dans le voisinage de l'image théorique, c'est-àdire de l'objet ramené à une distance $G$ fois plus petite.

Il suit de là que dès qu'on observe un objet tant soit peu éloigné, la convergence est toujours faible; les yeux ont tendance à se régler pour l'infini, c'est-à-dire dans la disposition la moins pénible. Il est donc prudent, pour réduire la fatigue au minimum, de s'efforcer de régler chaque lunette de manière à rejeter l'image le plus loin possible. Cela impose, le maximum de longueur de chaque corps (dans une lunette terrestre; le minimum dans la lunette de Galilée) compatible avec une netteté parfaite. Sans espérer atteindre le réglagè à quelques centièmes do millimètre près, il est toujours possible de ne pas amener les images aux petites dis- 
tances de vision distincte; car, de l'infini à la distance minimum de la vision ( 25 centimètres), la différence de réglage est perceptible. Pour l'instrument déjà considéré ( $\mathrm{G}=8, f=20$ millimètres $)$, la formule plus haut indiquée donne :

$$
\mathrm{L}=\mathrm{I}^{\mathrm{mm}}, 6 .
$$

II6. Jumelle à axes convergents. - Le parallélisme des axes, supposé jusqu'ici rigoureux, n'est jamais qu'approximativement réalisé : il est utile d'examiner les conséquences de cette imperfection.

Supposons d'abord que les axes restent dans un même plan; il peut y avoir alors soit convergence des axes en avant des objectifs, soit divergence (convergence en arrière des oculaires).

Considérons (fig. 35) une

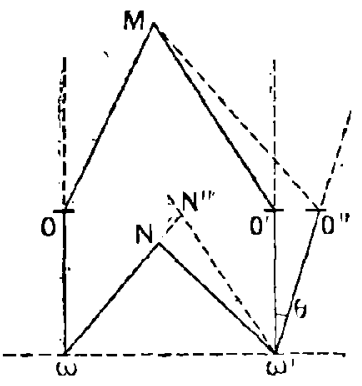

Fig. 35. jumelle à axes parallèles qui donne du point M l'image $\mathrm{N}$; admettons la constance du grossissement quel que soit le réglage des lunettes constituantes : cette hypothèse simplifie les calculs sans en modifier sensiblement les résultatș; elle est presque rigoureusement exactequand on rejette les images le plus loin possible.

Dans ces conditions, $\mathbf{N}$ est rapproché à une distance $G$ fois plus petite que celle de $M$; chaque lunette donne un faisceau émergent, tel $\omega \mathrm{N}$, $\mathrm{G}$ fois plus incliné sur l'axe que le faisceau incident correspondant, OM. Admettons maintenant que la lunette de droite $\omega^{\prime} \mathrm{O}^{\prime}$ 
tourne autour de $\omega^{\prime}$ d'un angle $\theta$, très petit d'ailleurs. Pratiquenent, l'angle $\mathrm{O}^{\prime} \mathrm{MO}^{\prime \prime}$ est négrligeable devant 0 . Le rayon incident central fait donc avec $\omega^{\prime} \mathrm{O}^{\prime \prime}$ un angle égal à ơ $+\theta$, et le rayon émergent $\omega^{\prime} \mathrm{N}^{\prime \prime}$ est incliné sur ce même axe de l'angle $G(0-\theta)$. La nouvelle image se produit donc en $\mathrm{N}^{-\prime \prime}$, el Ja figure donne sans difficulté la relation :

$$
\alpha^{\prime}=\alpha+(\mathrm{G}-\mathrm{I}) \boldsymbol{\theta}
$$

Si $\omega O$ s'incline à son tour, une même relation s'y applique; el la forme linéaire de celte dernière rend la formule unique, sauf que $\theta$ indique l'angle des axes convergent en arrière des oculaires.

Si la convergence a lieu en avant, les considérations précédentes conduisent à la même relation, à condition de donner le sirne moins à l'angle des axes.

La discussion de l'óquation ci-dessus n'offre aucune difficulté.

Quand les axes divergent (convergence en arrière), $\theta$ est positif; l'image se rapproche. Au lieu de se former à la distance $\frac{\mathrm{D}}{\mathrm{G}}$ correspondant à $\alpha$, elle se forme à la distance $\mathrm{E}$ répondant à $\alpha^{\prime}$, et la relation précédente conduit à :

$$
\mathbf{E}=\frac{\mathrm{G}}{\mathrm{D}}+\frac{(\mathrm{G}-\mathbf{I}) \boldsymbol{\theta}}{\mathrm{D}} .
$$

Le rapprochement esı très rapide : on en aura une idée en faisant $D=\infty$; supposant $\sigma=63$ millimètres et exprimant $\theta$ en minutes, l'équation donne, pour $\mathrm{G}=8:$

$$
\mathrm{E}=\frac{27}{9} \text { mètres. }
$$

Instruments optiques. 
Lne divergence d'une minute rapproche done l'image de l'infini à 27 mètres; avec un peu phus d'un degré, on atteindrait la distance minimum de la vision distincte.

Dans le cas de la conver crence, $\theta$ étant négatif, l'image s'éloigne; elle peut, même si elle était assez rapprochée, atteindre rapidement l'infini, et le dépasser (c'est-̀a-dire que les lignes visuelles convergent $\dot{a}$ l'intérieur de la tète; les yeux louchent en dehors).

II 7. - La conséquence immédiate rle ces résultats est que le non parallélisme des axes accroît les difficultés de la vision binoculaire. déjà délicate en raison de la faible tolérance sur le réglage des longueurs des lunettes. La convergence et la divergence des axes ont pour effet de tendre ì faire sortir les images monoculaires du champ de profondeur de l'weil correspondant; leur rôle s'ajoute à celui de la différence de longueur des axes.

La théorie ci-dessus indique des valeurs très faibles pour le maximum admissible de $\theta$. En supposant les lunettes parfaitement réglées et rejetant à l'infini, lorsqu'elles sont parallèles, les images monoculaires et binoculaires d'un point de l'infini, la divergence maximum admissible sera celleg qui ramènera vers i 5 mètres l'image binoculaire. On aura donc, en minutes :

$$
\theta=\frac{3000 \pi}{15(G-1)}=\frac{12,5}{(\dot{a}-\mathbf{I}},
$$

soit environ $2^{\prime}$ pour $G=8$.

La mème valeur convient à la convergence des axes.

I 18 . Jumelles à axes déversès. - Examinons maintenant l'effet d'un cléversement des axes; et, pour 
cela, supposons que, dans la jumelle à axes parallèles, $\omega^{\prime} \Omega^{\prime}$ tourne d'un angle très petit o autour de $\omega \omega^{\prime}$, en lui restant perpendiculaire. La ligne visuelle $\omega$ ' ${ }^{\prime}$ sort du plan qui contient $\omega$ N, car elle tourne, à très peu près, de $(G-1)$ q autour de $\omega^{\prime}$ en restant sensiblement dans un plan normal à celui des axes; le concours des lignes visuelles est impossible, géométriquement; il n'est assuré physiquement que si $\varphi$ est au plus égal à l'acuité visuelle. Le quotient d'une minute par G- I serait done le maximum de déversement admissible. Iu delà, la duplication de l'image devait șe produire.

La pratique ne justifie pas cette conclusion. Les images se maintiennent superposées avec des déversements plus considérables. Avec une jumelle de grossissement 7.5 , montée ad hoc, nous avons pu, sans entrâtnement spécial, maintenir la coïncidence des images en augmentant progressivement le déversement de zéro à une vingtaine do minutes. $\Lambda$ co moment, si on déplace légèrement les axes des yeux, la duplication s'opère, et me peut être réduite sans revenir à un déversement moindre.

La possibilité de maintenir en coincidence des imagres déversées ne paraît être susceptible d'explication qu'en faisant fonds sur la souplesse de l'organe visnel. En l'absence de tout repère dans le champ de la vision, les yeux se disposent de manière que les images réliniennes correspondant aux points images produits par les deux lunettes se forment aux centres des fovea. La sensation cérébrale devient ainsi unique, et le demeure tant que l'oil peut ainsi déverser ses axes.

Cette disposition anormale ne va pas sans fatigue. 
Quand la limite de ce strabisme dans l'espace est atteinte, le décalage des images se manifeste; les yeux, n'étant dès lors plus assujettis à la production d'une perception unique, reprennent leurs positions normales, et les deux imaces se séparent brusquenent.

Ir9. Fusion instable des images. - La même cause, disposition propice des yeux en l'absence de point de repère, peut seule expliquer la coincidence des images dans lo cas de convergence ou de divergence simples (sans déversement) très accentuées. Herschel (56) avait déjà remarqué que l'on peut obtenir la coïncidence d'une flamme de bougie et de son image ${ }^{1}$, produite par un prisme, d'angle variable à partir de zéro, interposé sur le faisceau allant de la flamme à l'un des yeux : la convergence des lignes visuelles est, d'après lui, susceptible d'atteindre no ou $30^{\circ}$ (normalement elle ne dépasse pas $15^{\circ}$ pour la distance minimum, 250 millinètres, de la vision distincte); la divergence serait plus faible; le déversement, peu appréciable.

Dans le même ordre d'idées, et avec la jumelle mentionnée plus haut, nous avons obtenu, toujours sans précautions particulières, une divergence ou une convergence des axes de I degré el demi environ, ce qui donne un angle des lignes visuelles voisin de $10^{\circ}$. Ajoutons que les jumelles étaient réglées de manière à reporter le plus loin possible les images individuelles.

Tant qu'il s'agit de jumelles ayant leurs axes dans un même plan, il semble que l'on peut assigner, comme lieu de l'image unique perçue, la rencontre des

1 Et méme de deux flammes réelles. 
DHS IMAGES DANS LES INSTRLMENTS BINOCLLARES 149 lignes visuelles. Il parait plus délicat de l'assurer (en prenant comme intersection la position de la perpendiculaire commune), lorsque les axes sont déversés.

r 20 . - Fous conclurons, de cette courte étude, qu'il est avantageux de rendre aussi parallèles que possible les axes d'une jumelle; mais que cependant, si des raisons, d'ordre économique surtout, s'opposent à un réglage trop précis, il n’est pas absolument nécessaire d'adopter les tolérances indiquées par la théorie physicogécométrique, trop rigoureuse. On peut, à notre avis, prendre pour la divergence ou la convergence des lignes visuelles une valeur voisine de $\mathbf{r}^{\mathbf{0}}$, et pour leur déversenuent, environ $3 o^{\prime}$. La tolérance sur la position relative des axes s'ensuit quand on connaît le grossissement.

I 2 . Champ de la vision binoculaire. - I.e non-parallélisme des axes a une influenco probable sur la netteté des images, ne fút-elle que la conséquence de la fatigue de l'oil; il diminue en outre le champ de la vision binoculaire.

Ce champ est la région commune aux champs individuels des lunettes, c'est-à-dire aux cònes ayant pour sommets les centres optiques des objectifs et comme ouverture l'angle $\gamma$ de champ. D'amplitude $\gamma$, - pour la vision à l'infini, 一 quand les axes sont parallèles, le champ binoculaire est réduit de $\sqrt{\theta^{2}+\varphi^{2}}$ quand les axes sont déversés de et convergent ou divergent sous langle $\theta$. Le champ n'est d'ailleurs plus exactement un cône de révolution, puisque sa base est la parlie commune à deux cercles égaux décalés angulairement de $\sqrt{\theta^{2}-\dagger Q^{2}}$.

132. Perception de la profondeur. - En 
ramenant à une distance $G$ fois plus petite que le point objet l'image binoculaire. une jumclle à axes parallèles en augmente dans le mème rapport

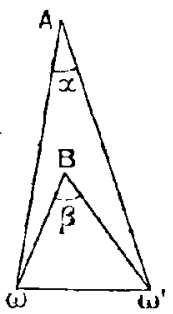

Fig. 36. la parallaxe visuelle. Elle facilite done la perception de la profondeur. Deux points $A$ et $B$ (fig. 36 ) qui, vus directement, ne seraient dislingués dans des plans différents que si

$$
\beta-\alpha \geqslant \mathbf{r}^{\prime} \text {, }
$$

- le seront, par leurs images $A^{\prime}$ et $\mathbf{B}^{\prime}$, si

$$
\beta^{\prime}-\alpha^{\prime} \supseteq \mathbf{I}^{\prime},
$$

c'est-à-dire, puisque

$$
\boldsymbol{x}^{\prime}=\mathrm{G} \boldsymbol{x}, \beta^{\prime}=\mathrm{G},
$$

si

$$
\beta-\alpha \geqslant \frac{1}{G} \text {. }
$$

Il existe un autl'e procédé, indiqué par IIelinholtz (53), pour amplifier les parallaxes: la figure 37 , où

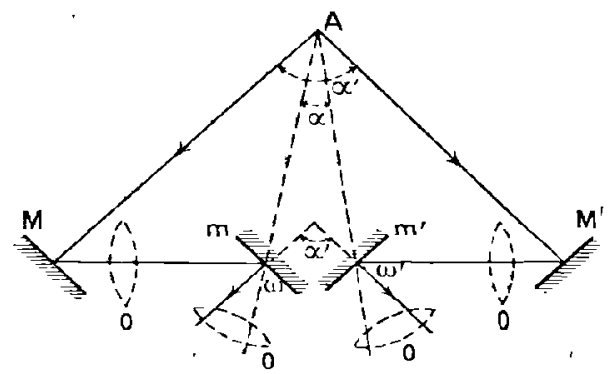

Fig. 37.

$\mathrm{M}, m$ et $\mathbf{M}^{\prime}, m^{\prime}$ sont deux systèmes de miroirs parallèles, le décrit suffisamment. Le point $\Lambda$, vu directe- 
ment par les yeux $\omega, \omega^{\prime}$, aurait pour parallaxe $\alpha$; perçu a travers le systéme, sa parallaxe est $\alpha^{\prime}$; et la géométrie donne aisément

$$
\alpha^{\prime}=x \frac{\pi}{\pi} \cdot=m x,
$$

$m$ étant le rapport des écartements des miroirs extrêmes et intérieurs (ce dernier intervalle égal à celui des yeux).

En disposant dans le système d'ITelmoltz des objectifs et des oculaires, firrurés en pointillé, de façon à constituer une jumelle à axes brisés, de grossissement $G$, la parallaxe $\alpha$ devient

$$
\alpha^{\prime \prime}=G m \alpha .=k \alpha .
$$

Le coefficient $k$ s'appelle le coefficient de profondeur.

La formule relative à la perception de la profondeur devient, lorsque les yeux s'aident d'un pareil instrument :

$$
\sigma=-200 \mathrm{G} m-p=-\frac{p^{2}}{200 k-p}
$$

La relation s'applique aux jumelles ordinaires, en y faisant $m=\mathbf{I}$.

ı 3. Téléstéréoscope. - Ln outre de l'augmentation de parallaxe, le dispositif d'Helmholtz amplifie la faculté stéréoscopique du système des yeux; car tout se passe comme si ceux-ci étaicnt reportés aux miroirs extrêmes : des récrions différentes de l'objet sont alors apercues, qui ne le scraient point par les yeux placós en $\omega$ et $\omega$, même armés d'une jumelle ordinaire. En munissant des lentilles mentionnées plus haut son système de miroirs, Ilelmholtz a pu appeler logiquement son appareil un télesstéroscope. II a fait (53), 
à ce sujet, une remarque qui mérite d'êlre signalée, surtout depuis que sur le modèle de cet instrument on a construit des systèmes varjés de jumelles stéréoscopiques.

Le système spéculaire d'Helmholtz, caractérisé par la valeur $m$, dont la signification a été indiquée plus haut, a pour effet de substituer à l'objet une image $m$ fois plus rapprochée, et clont les dimensions linéaires, sout $m$ fois plus petites que celles de l'objet, et cela dans tous les sens (axe VM' de l'instrument parallèle à la ligne des yeux, axe de profondeur normal à MI' et dans le plan des normales aux miroirs, direction normale à ces deux axes). Les propriétés des triangles semblables suffisent pour établir cette propriété. L'angle visuel, grandeur apparente de l'objet, se conserve dans l'image; mais la parallaxe des plans en profondeur étant amplifiée $m$ fois, l'image paraît $m$ fois plus allongée que l'objet.

Dans une jumelle ordinaire à axes parallèles, de grossissement $G$, l'objet est amené à une distance $G$ fois plus faible, les dimensions se conservant perpendiculairement aux axes. Ia grandeur apparente est $G$ fois plus grande, la parallaxe est $\mathbf{G}$ fois plus grande aussi; mais l'objet semble $G$ fois moins étendu en profondeur; car, amené matériellement à la distance de l'imagre, il aurait une parallaxe $G$ fois plus grande que celle-ci.

Lc dispositif d'IIelmholtz paraîl done allonger l'objet dans le rapport $m$; la jumelle, l'aplatir dans le rapport $G$ (ce dernier fait, bien appréciable dans les instruments d'usage courant). Il suit de là qu'un téléstéréoscope dans lequel on aura $m=G$ conservera à l'image l'aspect monoculaire et binoculaire de l'objet, 
qui sera vu comme s'il étail matériellement rapproché à une distance $\mathrm{f}$ fois plus faible que son éloignement réel. Telle est la remarque d'Helmholty, qu'il a d'ailleurs appliquée dans la construction de son appareil.

Dans ces conditions, la valeur $G$, égale à $m$, peut porter le nom de coefficient de relief.

r2/. Microscope binoculaire. - L'observation binoculaire, dans le microscope, procède d'un principe autre que celui qui régit la vision à l'airle de jumelles. L'objectif est, alors, unique; l'instrument porte deux oculaires à l'écartement des yeux.

La figure 38 indique l'une des dispositions employées. Sa théorie peut être exposée, comme il suit, d'après Helmholtz (53). Supposons le microscope mis au point sur le plan passant par le point aplanétique $P$. Lo système produira deux images ponctuelles $\mathbf{P}^{\prime}$ et $\mathbf{P}^{\prime \prime}$.

Un point $Q$ situé en arrière de $P$ donnerait, si le corps $O_{2}$ de l'appareil n'existait pas, une image qui, ponctuelle ou non, -ne serait pas dans le plan $\pi$ passant par $\mathbf{P}^{\prime}$ perpendiculaire à l'axe; l'oeil mis au point sur ce plan percevrait un cercle de diffusion concentrique à $\mathrm{P}^{\prime}$, intersection du plan $\pi$ et du faisceau réfracté par l'objectif. Dans le système binocu-

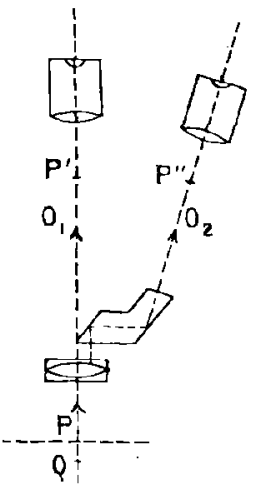

Fig. 3R. laire, la moitié seulement du faisceau arrive sur le plan $\pi$, et donne un demi-cercle de diffusion situé, dans le cas de la figure, à ganche de $\mathrm{P}^{\prime}$; l'autre moitié 
du faisceau produit un demi-cercle de diffusion, situé dans le plan $\pi^{\prime}$, à droite de $\mathrm{P}^{\prime \prime}$. Chacun de ces deux demi-cercles produit, si Q est suffisamment rapproché de P', la sensation d'un point image, dont l'weil rapporte la position au milieu du rayon du demi-cercle.

Les yeux faisant converger (fig. 39 ) $P^{\prime}$ et $P^{\prime \prime}$ en $P_{1}$, réunissent les deux innages $Q^{\prime}$ et $Q^{\prime \prime}$ au point $Q_{1}$, en arrière de $P_{1}$. La parallaxe relative des points $P_{1}$ et $Q_{1}$ dépend de $Q^{\prime \prime}$, qui est égal au rayon du cercle de diffusion. Si $f$ est la focale principale du microscope (systc̀ne total objectif et oculaire), $p$ et $q$ étant les distances de P' et de $Q$ au foyer antérieur du système, le rayon $\rho$ de cercle de diffusion est donné par la formule:

$$
p=f \frac{p-q}{p} \operatorname{tg} u
$$

$u$ étant la demi-ouverture de l'ohjectif du microscope, - l'xil est supposé placé all second foyer du système.

La différence des parallaxes de $P_{1}$ et $Q_{1}$ est sensiblement égale à :

$$
\beta=\frac{P}{\Delta}=\frac{\delta q}{f} \operatorname{tg} u,
$$

$\delta q$ étant l'écartement des points $\mathrm{P}$ et $\mathrm{Q}$.

On peut l'écrire, $\Delta$ étant la distance de vision des images:

$$
\beta=\frac{G}{\Delta}-\varepsilon q \operatorname{tg} u \text {. }
$$


DFS INAGES DANS LES INSTKLMENTS HINOCUIALRES 155 La profondeur minimum susceptible d'être perçue est donc, pour $\beta=\mathbf{x}^{\prime}$ et $\Delta=300$ millimètres :

$$
\Sigma q=\frac{1}{\operatorname{trggu}},
$$

soit, pour $\sin u=0,9$ et $G=500, \quad \delta q=0,1 \mu$. 


\section{CHAPITRE VIII}

IYSTHL MEYTS DE HESLRE

I 25. - Le caractère commun à tous les instruments optiques dé mesure est la faculté que leur donne leur organisation de pouvoir faire coïncider soit deux images entre elles, soit une inage avec un objet réel. Cette superposition exige d'un organe mécanique, lié à l'instrument, un déplacement ou une déformation, fonction connue de la grandeur à mesurer.

Quelle que soit la liaison mécanique en jeu, la précision de la mesure est d'abord en dépendance intime avec le degré d'exactitude de la coüncidence. C'est celleci qui doil être examinće.

I26. Coïncidence d'images réelles ponctuelles. - Ie rôle de l'instrument peut être de permettre la coürcidence de deux images réelles, soit par superposition intégrale des points correspondants, soit par juxtaposition de régions déterminées de ces images. C'est le cas général des télémètres purement optiques. La perfection de la coïncidence est jugée par un seul oil, armé, en général, d'un oculaire astronomique ou terrestre.

Le cas le plus simple est celui où le ou les objets 
qui fournissent les images sont des points lumineux, des étoiles, par exemple. Nous supposerons l'instrument organisé de façon que les petites innages solides aient mèmes dimensions. La superposition des images réelles sera appréciće d'après celle des images virtucllos données par l'oculaire, c'est-à-dire, en fin de compte, par les images rétiniennes.

Angulairement, la coïncidence des images virtuelles sera réputée parfaite si, ayant toutes deux un diamètre apparent inféricur à une minute, l'intervalle de leurs centres est au plus égal à l'acuité visuelle (une minute, également). Une minute est donc, dans ce cas, l'erreur maximum pratique qu'on peut raisomablement com mettre sur la cö̈ncidence des images virtuelless. Les caractéristiques de l'oculaire permettent d'en déduire l'erreur sur celle des images réelles. En considérant tout l'appareil, oculaire compris, comme une lunette de grossissement $\mathrm{G}$ pour chaque image, on voit que l'erreur possible est, angulairement, égale au quotient d'une minute par G, et, linéairement, à

$$
\frac{\mathrm{F}}{3000 \mathrm{G}^{-}} \text {, }
$$

F étant la focale du système qui a fourni l'une des images réelles.

Cette conclusion suppose que l'image virtuelle n'a pas de diamètre apparent sensible. Si'ce diamètre dépasse une minute et est égal à $\approx$ minutes, la coïncidence n'est plus appréciée qu'à $\equiv$ près, et par consćquent l'erreur linéaire sur la coïncidence des images réclles peut être égale à

$$
\frac{\mathrm{F} \varepsilon}{3000 \mathrm{G}} \text {. }
$$


En profondeur, il paraîtra y avoir superposition quand les images solides auront une partie commune, si faible soit-elle; car, dans ces conditions, la netteté peut être simultanénent maximum pour les deux inages. La règle s'applique tant aux inages virtuelles qu'aux images réclles. Elle vise plutôt l'organisation de l'appareil que son manienent. La différence de position en profondeur des imarres n'aurait, en effet, d'inconvénient que si elle permettait des

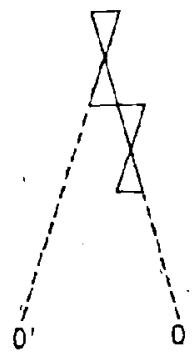

Hig. 40. coincidences diverses daus le sens latéral ou ançulaire, grrâce à la variation possible de position de l'cil : on sait que le déplacement de ce dernier est souvent utilisé pour la mise au point par utilisation de la parallaxe. Mais si les images perçues possèdent la netteté maximum, il ne pourra jamais y avoir sensation de parallaxe: La figure 40 lo démontre aisément : quelle que soit la position de l'mil susceptible de recevoir des rayons des deux faisceaux, l'écart angulaire des images virtuelles sera au plus égal à une minute : zéro pour l'cil placé en $O$, I' pour l'œil en $O^{\prime}$.

I 27 . Coincidence d'images de grandeur finie. - Lorsque les images à superposer ont des dimensions angulaires assez grandes, la précision de leur coüncidence diminue, à moins qu'on ne tourne la difficulté en se bornant à des détails assimilables à des points. C'est ce que l'on doit faire, dans la mesure du possible ; et les considérations précédentes s'appliquent encore. Sinon, la perfection de la coïncidence n'est jugée que par l'égalité de grandeur entre les images 
individuelles d'une part, et l'image résultant de leur superposition, d'autre part. Les erreurs possibles peuvent devenir considérables; il serait difficile, ailleurs que dans des cas d'espèce, d'en assigner les limites.

28. Juxtaposition d'images. - La précision de la juxtaposition de deux images est soumise à la mème règle que celle de la coincidence de deux points, si toutefois les lignes à confondre sont parfaitement neltes. Ce sont, en général, des droites plus ou moins longues, provenant ou non du même objet réel. Mais il est rare qu'on puisse atteindre la précision indiquée; car la différence d'aspect et de coloration des lignes, l'inégalité de clarté dans les images qui les fournissent, sont autant de causes qui atténuent, sans qu'on puisse en évaluer l'effet, la perfection du contact.

I 29. Coïncidence d'une image et d'un objet. - Ia coüncidence peut être demandée entre une image réelle et un objet. Le plus souvent celui-ci est constitué par une droite matérielle et ténue (fil de réticule, trait micmonétrique), ou le point de croisement de deux pareilles lignes; dans l'objet, s'il n'est pas ponctuel, on choisit un point net ou une arête bien tranchée.

Réduisant donc l'objet à un point, il s'agit d'appréeier la perfection de la coüncidence. Les considérations invoquées plus haut conduisent à la règle suivante.

Si le fil objet est opaque, le point image paraîtra en contact des que, dans la perception des images virtuelles données par l'oculaire, ił sera à une minute au plus de l'un des bords du fil, et en dehors de celui ci; si le trait objet est transparent, la coüncidence semblera exacte, dans les mèmes conditions angulaires, l'image étant alors entre les bords du trait. La précision est, 
dans co dernier cas, plus élevée que dans l'autre.

On constitue de pareils traits transparents soit en prenant comme repère l'intervalle compris entre deux fils opaques parallèles, soit en amenant l'image audessus du trait, supposé court, ou dans l'espace situé entre les deux portions (fig. 4x) d'un

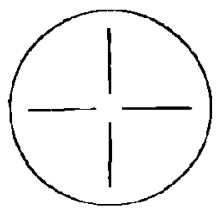

Fig. 41. trait interrompu. Alors, même quand les fils ou les traits sont larges ou que leur espace clair n'est pas très étroit, la précision est augmentée, si on rapporte la position de l'image au milicu idéal du trait ou de l'intervalle. L'ocil apprécie avec assez d'exactitude cette ligne fictive, et l'on peut assurer sa coüncidence avec l'image à une minute près. La pratique justific assez bien cette règle.

r3o. Mise au point d'un instrument à réticule. - Lorsqu'on veut réaliser la superposition d'un point de l'image réelle et d'un fil de réticule, la première opération consiste à obtenir Ia coïncidence en profondeur par déplacements convenables de l'oculaire par rapport au réticule, et de l'ensemble relativement au système objectif. Un procédé assez employé utilise, à cet effet, la parallaxe optique qui se manifeste, quand on déplace légèrement l'ceil, entre le fil de réticule et l'image, lorsque leur coïncidence n'est pas établie. Le colonel Goulier (39) trouve cette façon d'opérer insuffisante, susceptible seulement d'application aux lunettes médiocres non achromatiques, et cela à cause de la faculté d'accommodation de l'œeil, qui s'opère inconsciemment. Il recommande de mettre d'abord au point le micromètre pour la vue la plus facile de l'observa- 
teur, la lunette dirigée sur le ciel, et ensuite, sans s'occuper du micrometre, de disposer l'ensemble de manière à obtenir la netteté maximum du point visé; dans cette opération, d'ailleurs, Ies déplaccments doivent itre assez rapides pour que l'weil ne puisse s'accommoder, et le système mobile doit osciller autour de la position définitive de plus facile visibilité.

Les considérations développées plus haul peuvent justifier cette rè gle.

I3 . Coïncidence d'une image virtuelle et d'un objet. - Il est possible de faire coïncider une image virtuelle avec un objel róel lorsque les faisceaux qui en émanent sont reçus par des régions différentes de la pupille (généralement, les deux moitiés). La précision de la superposition angulaire ne saurait alors être mesurée par moins d'une minute; pour l'atteindre, il faut que l'image se trouve dans le champ de profondeur de l'œil mis au point sur l'objet, et que cette image soit sensiblement ponctuelle dans sa partie efficace.

I 32. Coïncidence successive en profondeur. - La coïncidence en profondeur peut être utilisée comme procédé de mesure, même quand la superposition angulaire n'est pas assurée. L'emploi du microscope et de la loupe est assez fréquent à cet effet.

Deux cas principaux peuvent se présenter.

Le premier concerne la mise au point successive sur deux images; on amène ainsi, à des époques différentes, les pinceaux émergeant de l'instrument à venir converger sur la rétine. Si, sur cette dernière, l'image d'un objet ponctuel était un point susceptible de n'être perçu tel que pour une accommodation bien déterminée de 
l'ooil, la sensation de netteté parfaite serait corrélative de la situation de l'objet par rapport au système optique. Ces conditions n'ćtant pas réalisées, il importe de déterminer l'erreur sur la position des objets qui peut résulter d'une mise au point en apparence exacte.

La mise au point d'un système (S) sur le poinl objet P' de son axe (fig. 42) est assurée quand l'image

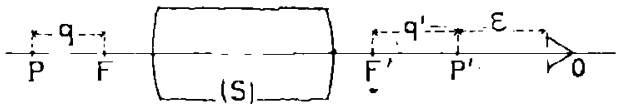

Firs. 42.

virtuelle ou réelle $\mathrm{P}^{\prime}$ se trouve dans le champ total de profondeur de l'weil, c'est-à-dire entre l'infini et la distance minimum $\Delta_{*}$ de la vision distincte. La variation possible de $\mathbf{l}$ 'est celle qui provient du déplacement possible de $\mathrm{P}^{\prime}$. La figure ci-dessus donne, en désignant par $n$ l'indice du milieu dans lequel est plongé le point objet :

$$
q q^{\prime}=f f^{\prime}=f_{n}^{2}
$$

et comme $q^{\prime}$ peut varicr entre $\propto$ et $\Delta_{0}-\varepsilon$, la variation de position $2 q$ de $\mathrm{P}$ a pour expression :

$$
z q=\frac{f^{2}}{n\left(\Delta_{0}-\varepsilon\right)} \text {. }
$$

Dans la loupe ordinaire, $(n=1$, et $\varepsilon$ négligeable dès que l'instrument est un peu puissant) la formule se réduit à

$$
\partial q=\frac{f^{2}}{\Delta_{0}}=\frac{f^{2}}{250} \quad \text { [unité: millimètre } \text {. }
$$

Une loupe de 10 millimètres de focale n'assure donc la mise au point qu'à 4 millimètres près. 
Pour le microscope, de grossissement G, l'équation

$$
\mathbf{G}=\frac{\Delta_{0}}{f}=\frac{n \Delta_{0}}{f},
$$

conduit, en négligeant $\varepsilon$, à la formule :

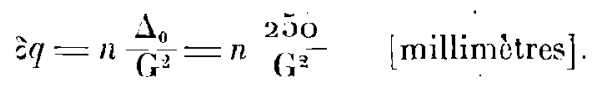

Pour obtenir, dans l'air $(n=\mathrm{I})$, la précision da

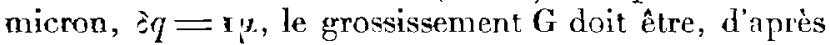
l'équation ci-dessus, au moins égal à 500.

33. Coïncidence simultanée en profondeur. - Lorsque la coincidence en profondeur s'opère simultanćment pour les deux images, ou lorsqu'on fait usage d'un réticule dorat on assure la coïncidence successive avec chaque image. la précision augmente.

Si deux objets sont observés simultanément à travers le système (S), la coïncidence en profondeur semblera encore exister quand leurs images solides se toucheront par leurs bases (fig. 43). Admettons, pour simplitier les calculs, que l'ceil se trouve au second foyer du systène.

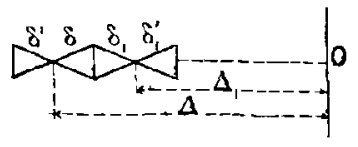

Fig. 4.3. Les équations suivantes résolvent la question (voir $\$$ I 1 / ) :

$$
\begin{aligned}
& q_{1} \Delta_{1}=q \Delta=f^{2} \\
& \Delta-\Delta_{1}=\dot{c}+\tilde{s}_{1}^{\prime}, \quad \delta=\frac{\Delta^{2}}{15+\Delta}, \quad \dot{j}^{\prime}=\frac{\Delta_{1}^{2}}{15-\Delta_{1}} .
\end{aligned}
$$

L'erreur possible $\varepsilon$, différence entre $q$ el $q_{1}$, prend la forme :

$$
\varepsilon=-\frac{2}{15} f^{z} \quad[\text { unité : mètre }]
$$


Comparant avec la formule du numéro précédent, on voit qu'ici l'erreur est 3o fois plus faible.

Quand la coüncilence a lieu à l'aide. dlu réticule, la mème relation s'applique; mais l'erreur ćlant possible dans les denx sens, il faut, évidemment, en cloubler la valeur pour obtenir l'erreur possible entre les deux points dont les images sont successivement amenćes sur le réticule.

\section{I31. Coïncidence en profondeur et netteté.} - Les calculs sommaires relatifs à la coïncilence en profondcur supposent le système aplanétique et la pupille normalement ouverte.

Quand cette dernière se ferme, ou, ce qui revient au même, lorsque l'annean oculaire du système est étroit, la profondeur de champ, pour une distance donnée, augmente. On sait, en effet, qu'en regardant à travers un petit trou, la distance de la vision distincle diminue (avec un trou de $\mathrm{O}^{\mathrm{mm}}$, I on arrive à lire l'ćcriture à 3o millimètres); il s'ensuil que, dans ces conditions (le fait se frésente avec les microscopes à fort grossissement dont les anneaux oculaires sont très petits), la précision de la mise au point peut s'affaiblir. Il est difficile de dire dans quelle proportion.

La profondeur de champ diminue, au contraire, à mesure que l'aberration sphérique augmente, l'image solide devenant alors, de conique, hyperboloïdale. Il en résulterait que la précision de mise au point s'accrồtrait. La conséquence n'est pas niable. Il ne faudrait pourtant pas annuler la profondeur de champ; car la mise au point deviendrait mécaniquement délicate, les images fatigueraient l'œil, qui se contenterait inconsciemment d'une netteté moindre." 


\title{
DEUXIÈME PARTIE
}

\section{ORGANISATION ET EMPLOI DES INSTRUMENTS}

\author{
GHAPITRL: IX \\ IYSTRUMEYTS D'OBSEHVATIOX A GRANDE DISTAYCE
}

\section{Lunet te astronomique.}

I35. Organisation de la lunette astronomique. - La lunette astronomique est constituée par deux systèmes convergents, objeclif et oculaire, dont les foyers en regard sont voisins l'un de l'autre. Cet ensemble donne, d'un objet situé au delà clu foyer antérieur de l'objectif, une innage définitive virtuelle qui est renversée, c'est-à dire qui se présente à l'observateur comme si l'objet avait tourné de $180^{\circ}$ autour de l'axe de la lunetle.

L'oculaire est mobile, suivant l'axe, pour la nise au point de l'instrument, laquelle consiste à amener l'image virtuelle dans le champ de profondeur de l'œil. Celuici se place de façon que sa pupille apparente soit centrée sur l'anneau oculaire et dans son plan. 
L'anneau oculaire est réel; il est l'image, à travers la lunette, de la surface extérieure de l'objectif.

L'instrument comporte un seul diaphragme de champ placé au droit de l'image réelle, et des diaphragmes de clarté. Il ne renferme aucun diaphragme do limitation; dans les appareils bien construits, la monture extérieure de l'objectif en lient lieu.

Ia Iunclte astronomique est utilisce pour l'observation des corps célestes, le renversement des images n'ayant ici aucun inconvénient, puisque les notions de haut, de bas, de droite et de gauche n'ont aucune importance; elle est employée pour l'examen des objets terrestres éloignnćs quand l'obscrvateur' se décide à subir le renversement des images; enfin, munie d'organes spéciaux (réticules ou micromètre), elle se transforme en instrument ou organe de mesure.

r36. - Ia figure 44 indique l'organisation schóma-

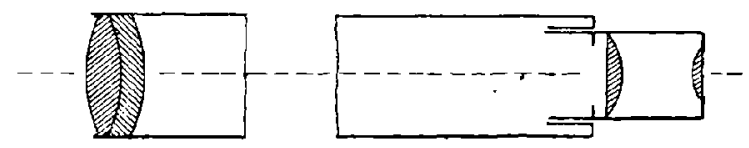

Fig. 44 .

tique d'une lunette astronomique; l'oculaire figuré est celui de Ramsden.

137. Lunettes non achromatiques. - La première lunetle astronomique, celle de Kepler, imaginée vers e 6 r 5 , consistait uniquement en deux lentilles, simples, convergentes. Huygens y apporta, en $\mathbf{1} 669$, une première modification en remplaçant la lentille oculaire simple par une combinaison de deux lentilles 
convergentes non contiguës. Le rồle de ce système double était d'augmenter le champ, à ouvcrture égale, des lentilles d'oculaire, et de réduire l'aberration sphérique que comportait la Ientille unique.

L'aberration sphérique de l'objectif était peu à considérer; car le diamètre du cercle d'aberration sphérique n'était que le $\frac{\text { I }}{5000}$ environ de celui du cercle chromatique; l'oculaire simple avait plus d'influence, dès que le champ s'agrandissail, car les rayons tombaient sur ses surfaces sous des angles considérables.

Ie défaul des lunetles résidait principalement dans les irisations des images. Hcureusement, le diamètre du cercle d'aberration chromatique ayant pour expression, dans le cas de la vision d'objets ă l'infini :

$$
\Omega \frac{\partial y}{\mu-I}
$$

$(\Omega$, ouverture de l'objectif ; $\mu$, indice moyen; ô, dispersion), est indépendant de la focale de l'ubjectif. Fn augmentant cetle focale, et par suite, pour un oculaire déterminé, le grossissement, on peut réduire la proportion de la largeur du bord de coloré (qui reste constante) par rapport au diamétre de l'image, qui s'agrandit; d'autre part, l'oculaire simple jouit sensiblement de l'achromatisme apparent; l'oculaire d'Huygens possède la même propriété, à un degré plus élevé. On utilisait ces remarques, avant la découverte de l'achromatisme, pour augmenter la netteté des images. C'est la raison qui a fait porter les lunettes de l'époque à des longueurs de 30 à 50 mètres.

La possibilité d'obtenir des systèmes achromatiques 
a permis de réduire la longueur des instruments, pour une netteté donnée. Dès Jors la lunette est constituće par un objectif possédant, plus ou moins complètenient, l'achromatisme absolu, et par un oculaire assurant avant toul, suivant l'indication d'Euler, la dispersion rectiligne des foyers, c'est-à-dire l'achromatisme apparent du système total.

I38. Lunettes achromatiques. - Objectif. La plupart des objectifs de lunettes astronomiques comprennent une lentille convergente, extérieure, en crown et, tout près d'elle, sinon au contact, une lentille divergente en flint; le crown est le plus souvent biconvexe, la face extérieure plus aplatie que l'autre; le flint a la forme d'un ménisque se rapprochant dı plan concave; les faces en regard des deux verres ont des courbures voisines ${ }^{1}$.

Les deux types les plus courants sont celui de Cilairaut (12) et celui de Fraunhofer. Dans les objectifs qui réalisent la condition de Clairaut, - égalité des

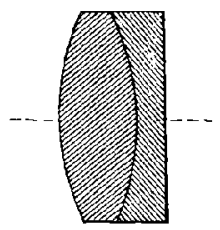

Fig. 45 . courbures des faces en question cidessus, - les denx verres (fig. 15) sont appliqués l'un sur l'autre et souvent collés au baume de Canada, au moins quand leur diamètre ne dépasse pas 50 à 60 millimètres. Les trois rayons de courbure des surfaces réfringentes de l'objectif sont. déterminés de façon à donner à ce dernier une focale déterminée, à réaliser pour deux radiations (D, F, en général) l'achromatisme absolu pour un pinceau

1 Cauchois avait essayé jadis de substituer le flint au crown et le cristal de roche an flint (brevet français $n^{\circ} 3688$ ). 
parallèle centré sur l'axe, et à obtenir l'aplanétisme central (coïncidence des foyers dus aux rayons centraux et marginaux) pour un faisceau parallèle à l'axe ct de couleur définic par la raic $D$.

Dans les excellents objectifs astronomiques construits par Fraunhofer, d'après des principes qu'il n'a pas divulgués, les faces en rerard (fig. 46) ont des courbures presque égales, la face du flint étant toutefois plus aplatie que celle du crown; les deux lentilles sont en contact sur l'axe. D'après l'étude de ces objectifs, il semble qu'ils réalisent, en plus des conditions optiques du type de

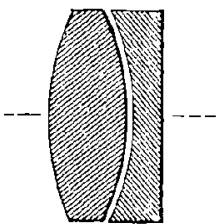

Fig. 46. Clairaut, l'aplanétisme central pour un faisceau émanant d'un point de l'axe et situé à 40 fois environ la focale de l'objectif (67). Une condition presque identique a été indiquée par Herschel pour la construction des objectifs à deux verres.

On rencontre encore des objectifs qui sont voisins du type de Clairaut, en ce sens que les surfaces en regard. sont presque au contact et possèdent sensiblement les mêmes courbures; mais à l'inverse du type de Fraunhofer, la courbure du flint est un peu plus forte que celle du crown. Cette disposition a uniquement pour but de produire le contact des lentilles par leurs bords, lorsqu'on tient à éviter le collage au baume ou l'emploi d'une rondelle d'appui pour la lentille arrière. L'intervalle qui existe entre les lentilles est maintenu juste suffisant pour qu'il ne se produise pas d'anneaux colorés.

Bien d'autres combinaisons ont été proposées ou Instruments optiques. 
expérimentées pour réaliser dans l'objectif à deux verres une quatrième condition optique, en dehors de celles que possède le type de Clairaut; et cela, en n'assujettissant pas les lentilles à être en contact intime.

D'Alembert avait déjà indiqué la condition de l'aplamétisme central pour une seconde radiation. Canss a calculé sur cette base un objectif, auquel on a depuis altaché son nom, et qui est aplanétique pour le rouge et le violet. Construit par Steinheil, avec les données de Ciauss, la combinaison a été trouvée défectueuse (36). De meilleurs résultats semblent avoir été obtenus, en employant d'autres verres, par Steinheil et par Krüss. L'objectif de l'équatorial de l'observatoire de Princeton (États-Unis) aurait été calculé par Clark sur to principe de Gauss.

Klïgel avait proposé, sans raisons péremptoires, de donner à la lentille de crown la forme équiconvexe; plus tard, d'assurer dans le crown la déviation minimum du faisceau marginal parallèle à l'axe (67).

La solution d'HIerschel, analogue à celle de Fraunhofer, est de rćaliser l'aplanétisme pour un point situé à une distance de l'objectif telle que, dans les calculs, on puisse négliger le carré du rapport de l'ouverture de l'objectif à cette distance. Des tables, calculées dans cette hypothèse, ont été reproduites par Pretchl, et répandues dans la pratique en Allemagne.

Plus récemment, on a étudić des objectifs soit à plus de deux lentilles, soit comportant des verres autres que le crown ct le flint habituels. Déjà le colonel Goulier avait employé, pour réduire la focale avec une ouverture donnée, deux objectifs du type Clairaut accolés. Reprenant l'une des idées de d'Alembert, 
M. Ilarling (49) a calculé un objeclif comprenant une lentille divergente en flint comprise entre deux lentilles convergentes en crown, les faces en regard étant appliquées, avec ou sans baume, l'une sur l'autre.

Ia variété des nouveaux verres introduits dans l'industrie optique depuis $\mathbf{1} 886$ a permis la réalisation d'objectifs du type Clairaut possédant en outre la propriété d'être aplanéliques pour un point déterminé de l'axe (condition Herschel ou Fraunhofer). C'est par le choix des verres constituants qu'on parvient a satisfaire aux quatre équations de condition ne renfermant que trois rayons de courbure. M. Ilarting $(47,48$, 50) et enslite M. von Hoegh (58) (des Etablissements Goerz, à Friedenau) ont, par des méthodes différentes, étudié le problème.

Signalons enfin l'emploi de surfaces non-sphériques dans la construction des objectifs, afin de leur donner le maximum de qualités; procédé utilisé par Alvan Clark dans les gros objectifs astronomiques, indiqué à nouveau par II. Schröder (36), puis par les Établissements Zciss (d'Iéna) (80).

г 39 . Oculaire. - L'oculaire primitif de la lunette astronomique était une lentille simple. Il n'a jamais été entièrement abandonné. Biot a démontré, - le fait était connu des opticiens, - que pour les forts grossissements, il n'y a que des avantages à employer des oculaires simples, qui font d'ailleurs perire moins de lumière par réflexion. Lin tel oculaire est constitué par une lentille plan-convexe, la face plane à l'arrière, l'ouverture étant au maximum les $\frac{3}{10}$ du rayon de la face antérieure, ou bien, quand ce rayon est inférieur à 
I millimètre, par une demi-sphère, ou entin, par une sphère de rayon très faible. - Ainsi IIerschel a employé une splıère de $o^{\mathrm{mm}}, 2$ 'l environ, donnant un grossissement de 6000 avec un objectif de $2^{\mathrm{n}}, \mathrm{x} 64$ de focale et i 6 centimètres d'ouverture. - Les oculaires simples restreignent considérablement le champ; ils donnent un point oculaire situé en avant de leur surface arrière, et, par suite, n'assurant pas, dans de bonnes conditions, la dispersion rectiligne des foyers, ils ne conviennent qu'à des observations axiales.

Les deux oculaires types de la lunette astronomique sont des combinaisons de deux lentilles plan-convexe, simples, taillées dans un même crown, et écartées l'une de l'autre.

Le premier système est l'oculaire d'Ilaygens (fig. 47), dit aussi de Campani, ou oculaire

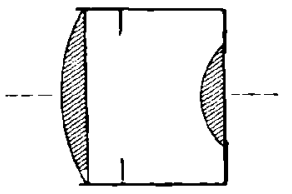

Fig. 47. négatif; les deux surfaces convexes sont tournées vers la lumière. Ia lentille antérieure, dite collectrice ou verve de chaimp, placée en avant de l'image objective, rend celle-ci virtuelle, et lui substitue une image réelle, plus petite, et moins éloignée de l'objectif.-La lentille arrière, ou vere d'cil, joue le rôle de loupe par rapport à l'image réelle.

Les caractéristiques de loculaire d'Huygens sont les suivantes, les lentilles étant supposées infiniment minces :

$$
f=4, \quad f=\frac{4}{3}, \quad b=\begin{aligned}
& 8 \\
& 3
\end{aligned},
$$

pour une focale totale $\rho=\mathrm{I}$. 
$\left(f, f^{\prime}\right.$ focales respectives des lentilles avant et arrière, $b$ intervalle des verres).

Ces données réalisent l'achromatisme apparent, assurent ì la lunette un anneau oculaire réel. La disposition convexe des faces vers la lumière a pour hut de diminuer les aberrations de sphéricité.

Ramsden a proposé le second système, qui n'est autre qu'une loupe composée, à deux verres, avec laquelle on observe l'image réelle. I.cs deux lentilles simples ont ici leurs convexités en regard (fig. 48).

Les caractéristiques de l'oculaire de Ramsden, - appeló aussi positif, celui d'Huygens étant dit nég̨atif,

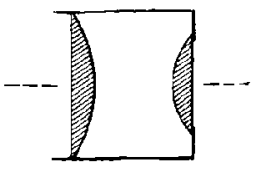

Fig. 48 . - sont les suivantes $(8)$ :

$$
f=\mathrm{I}-u^{2}, f^{\prime \prime}=\frac{\mathrm{I}-u^{2}}{\mathrm{I}-2 u^{2}}, b=\frac{\left(\mathrm{I}-u^{2}\right)^{2}}{\mathrm{I}-2 u^{2}},
$$

$u^{2}$ étant plus petit que o, $\overline{5}$.

Ces données, qui assureraient la dispersion rectiligne des foyers, conduiraient à un anneau oculaire situé en avant du verce d'oeil. On évite cet inconvénient en sacrifiant l'achromatisme apparent, et en adoptant les valeurs :

$$
f=f^{\prime}=-\frac{4}{3}, \quad b=\frac{1}{3}\left(f+f^{\prime}\right)=\frac{8}{9},
$$

pour $\varphi=\mathbf{r}$.

Les oculaires de Ramsden et de Iluygens du type normal ne donnent qu'un champ apparent maximum de $0, f \circ$; il n'est pas d'ailleurs entièrement utilisable, en raison de sa courbure et de la distorsion des images, distorsion plus accentuée dans l'oculaire négatif que 
dans l'autre. Lorsque le rôle de la lunette exige mieux que la seule netteté au centre du champ, les qualités des oculaires en question sont dévéloppées, grâce ḋ la substitution, partielle ou totale, de lentilles composées aux veries constituants.

Karl Kellner (65) avait déjà, dans son oculaire orthoscopique (fig. 49), modifié l'oculaire d'Iluygens, en

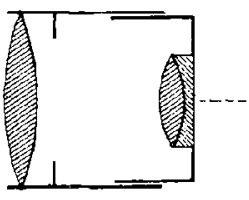

Fig. 49. remplaçant le verre d'wil par une lentille crown-flint, et en donnant au verre de champ la forme biconvexc. La même modification a été, depuis, apportće au verre d'ceil de l'oculaire de Ramsden, par divers opticiens, en vue de détruile aussi parfaitement que possible l'aberration chromatique et de corriger la distorsion ${ }^{1}$. Plus récemment, pour remédier à la courbure du champ et à l'astigmatisme, le verre d'œil composé s'est augnenté d'une lentille simple accolée ou non à lui.

Le colonel Goulier avait constitué l'oculaire positif' de son tachéomètre du génic par deux lentilles doubles crown-flint. Dans l'oculaire de

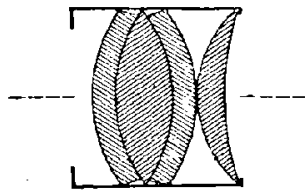

rig. Gundlach, le verre collecteur est clouble, le verre d'neil triple (36).

On a également constítué des aculaires composés uniquement de lentilles jointives, collées on non. 'Tel est l'oculaire euryscopique de Mittenweg (fig. 5o), à

1 Zerss. Brevets fr. 368043-375748.

IRIS - LILLIAD - Université Lille 1 
INSTRUMENTS D'ORSERVATION A GRANDE DISTANCE 175 quatre verres, et l'oculaire monocentrique de Zeiss, à trois lentilles accolées (36).

Dans ces divers oculaires à plus de deux lentilles simples, les focales des verres n'out plus entre elles des relations simples comme dans les types normaux. Les courbures des faces dépendent de la nature des matières employées et du but poursuivi. On se bornera à indiquer l'un des progrès rúalisables : le champ apparent peut être assez aisément porté à 0,60 , et même atteindre 0,75 , sa courbure étant négligeable, et la netteté des imagres conveuablement assurée.

I ́́o. - Parmi les lunettes astronomiques, les plus intéressantes sont celles qui sont utilisées pour les observations célestes. Elles se caractérisent par la grande amplitude de leur ouverture. Le diamètre de l'objectif, qui ne dépassait pas 40 centimètres au milieu du $x_{1} x^{\theta}$ siècle, a été progressivement augmenté, et a atteint ro5 centimitres (observatoire de Yerkes); il a été prévu à $\mathbf{2} 25$ centimètres dans la grande lumette

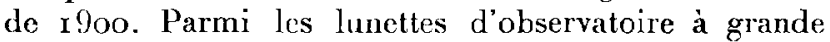
ouverture, on citera encore : 7/4 (Paris); 77 (Nice); 80 (Potsdam); 8 I (Pulkowa); 8/ (Meudon); 88 et $9_{7}$ (Lick). Ces nombres expriment, en centimcitres, les diamètres d'objectif.

L’objectif est généralement du type Fraunhofer. Certains spécimens, - objectifs astrophotographiques, qui doivent être aussi utilisés pour la photographie céleste, sont corrigés visuallement et actiniquement. Parfois la lunette comporte deux objectifs, I'un pour les observations, l'autre pour les opérations photographiques.

Le rapport de la focale à l'ouverture, jadis voisin 
de $\mathbf{1} 2$, a élé porté à $\mathbf{1} \bar{j}$, puis à 25 , et prévu à 40 dans la grande lunette de i goo. Le bulde cette augmentation a été d'accroitre les dimensions absolues de l'image objective, ce qui présente un ccrtain intérctt pour la photographic.

Les grossissements employés dans ces lunettes dépassent, très largement parfois, le grossissement normal. La pénétration ne varie pas, mais l'observation est facilitée, ot la clarté peut ètre accrue s'il s'agit d'étoiles. D'ailleurs, la question de l'amplitude du champ peut, dans des cas très étendus, être négligée.

\section{Lunette terrestre.}

I 4 I. - Le rôle de la lunette terrestre est de donner d'un objet éloigné nne image définitive virtuelle, celle qu'observe l'cil, droile par rapport à l'objet, c'est-àdire ayant la mêrne orientation que lui relativement à la direction de la vision.

L'optique connaît deux types de cet instrument : le premier, auquel on réserve, le plus généralcment, le nom de lunelle terrestre sans qualificatif ou de longuevuc, est constitué par une lunette astronomique, dans laquelle un système optique opère le redressement de l'une des images; le second est la lunette de Galilie, -plus souvent employée dans les jumulles, - dans laquelle l'oculaire redresse l'image objective en la rendant virtuelle avant sa formation.

I/2. Longue-vue de Kepler. - La plus ancienne longue-vue est celle de K' lentille unique remplit le rôle du véhicule; olle prend 
INSTRUMENTS DOBSERVATION A GRANDE DISTANCE 177 l'image objective $a$ réelle et renversée, et, la laissant encore réelle, la transporte en $a^{\prime}$ en la redressant. Cette seconde image est observée par un oculaire astronomique qui la rend virtuelle.

La lentille véhiculaire se place en général de façon que sa distance à l'image objective soit le double de sa focale propre; le transport de l'image s'opère sans amplification; mais, ce qui est le plus important, la disposition donne à la fois le minimum de longueur à

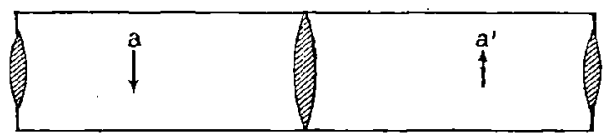

Fig. 51 .

la lunette, et, aux verres, les plus petites ouvertures, les focales étant données.

Les inconvénients du système tiennent d'abord au trop grand développement de l'instrument : à la longueur de la lunette astronomique, s'ajoute en effet le quadruple de la focale du véhicule, qui, à cause de l'ouverture du faisceau, ne saurait être faible, si la lentille est simple. La grandeur de la focale du véhicule oblige à donner à cette lentille une grande ouverture, pour mieux corriger lesaberrations, et aussi pour permettre que les faisceaux provenant des points du champ réel l'atteignent; sinon l'objectif ne travaillerait pas dans toute son ouverture, qui ne correspondrait plus ainsi au grossissement. D'autre part, la simplicité du système a pour conséquences la distorsion des images et la courbure du champ. 


\section{Organisation de la longue-vue à vẻhi-} cule. - La rédnction de longueur de la lunette précédente peut s'effectuer en remplaçant la lentille véhiculaire unique par un système de deux lentilles écartées, dont la première, - lc collecteur, - est près de l'image objective, et dont l'ensemble a un foyer en arrière de colle-ci. Le rapprochement de la lentille vers l'inage diminue l'ouverture nécessaire du premier verre, cette ouverture étant la section de l'ensemble des faisceaux réfractés par l'objectif, et qui divergent de l'image objective. lin écartement convenable du second verre, le redresseur, lui pernet, avec une ouverture au plus égale à celle du collecteur, de recevoir les faisceaux réfractés par ce dernier et ayant coupé l'axe entre les deux lentilles du véhicule. La réduction des ouvertures amène celle des focales. el par suite de la longueur de l'instrument.

Les théories actuclles cle l'optique géométrique déterminent aisément la meilleure disposition à donner au véhicule aussi conçu. Il n'est pas probable que le P. Rheita ait suivi cette voie. Peut-être eut-il l'idée d'examiner l'image objective avec un microscope, connu à son époque, ou, plus vraisemblablement, avec une seconde lunette astronomique : l'objectif de celle-ci constitue, avec l'oculaire de la promière, le véhicule. Err faisant varier la distance de ces verres, on peut déterminer expérimentalement la meilleure valeur à lui donner en vue de la production du champ maximum. Ces remarques expliqueraient. l'organisation du véhicule indiquée par certains traités d'optique : égalité des focales des verres, coincidence de leurs foyers avec les deux images réelles formées dans l'instrument. 
En modifiant cette disposition véhiculaire de manière à rapprocher le plus possible le verre collecteur de l'image objective, el en substituant à l'oculaire simple la combinaison d'Ifuygens, l'opticien Dollond a donné à la lunette terrestre sa forme définitive.

La figure 52 en présente l'organisation schématique.

Bien qu'au fond le rôle véhiculaire de la lentille unique de la lunetle primitive soit ici rempli. par trois verres, c'est à l'cnsemble des deux premiers qu'on a laissé le nom de véhicule. Le système des quatre derniers verres porte le nom d'aculaire terrestre : celui-

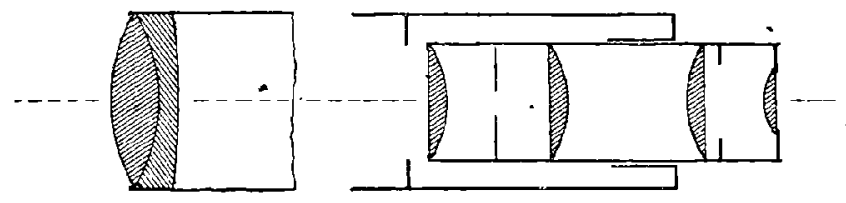

Fig. 52.

ci comprend donc le véhicule et l'oculaire astronomique, du type lluygens, en vue de l'augmentation de champ, à moins que les conditions d'emploi de l'instrument n'indiquent l'oculaire positif.

L'annean oculaire est réel et éxtérieur à l'appareil. La mise au point s'effectue par déplacement soit de l'ensemble de l'oculaire terrestre, soit du verre d'oil seul (ou de l'oculaire de Ramsden, le cas échéant), soit des deux systèmes composants, en génćral : donc on met au point sur les images réelles.

La lunette comporte, en dehors des diaphragmes de clarté, deux diaphragmes de champ dans les plans des 
images réelles, quand l'ohjet et l'image virtuelle dernière sont à l'infini : plan focal de l'objectif et plan focal du verre d'osil. En outre, un diaphragme de limitalion est disposé dans le plan où les faisceaux réfractés coupent l'axe à l'intérieur de l'instrument: ce plan est l'image, situće entre les verres du véhicule, de la surface antérieure de l'objectif par rapport à l'ensemble de l'objectif et du verre collecteur.

I 14. Objectif. - L'objectil de la lunette terrestre est identique à celui de la lunette astronomique. En général, on emploie le type de Clairaul ou celui de Fraunhofer. Sauf circonstances spéciales, les considérations économiques font rejeter les types complexes et les verres autres que le cronn et le flint.

I 45. Oculaire terrestre de Dollond. - L'oculaire terrestre normal, celui de Dollond, comprend quatre lentilles plan-convexe, cn crown, placées conme l'indique la figure 52. La disposition relative des faces courbes a pour effet de réduire les aberrations de sphéricité. Les valeur's relatives des focales et des intervalles des lentilles assurent, outre les conditions géométriques (grossissement, position de l'anneau oculaire, champ), la réalisation de l'achromatisme apparent et sa stabilité. Dans un travail remarquable (9), Biot a étudié longuement ces dernières questions, et en a appliqué les conclusions à l'examen d'oculaires existants construits par Dollond, Ramsden et Fraunliofer. Voici les caractères les plus simples de l'oculaire terrestre, tel qu'on le rencontre dans les longues-vues ordinaires.

Les focales des lentilles du véhicule sont égales ou peu différentes; - celle du redresseur est la plus grande; leur rapport varie de I à $\mathrm{I}, 25$; l'intervalle des verres 
est cnviron les $\frac{2}{3}$ de la somme des focales; le grossissement propre du véhicule est voisin de $\mathbf{2}$.

L'oculaire astronomique négatif n'a généralement pas les constantes indiquées plus haut. Le rapport des focales des verres, au lieu d'être 3 , varie de $x, 5$ à 2 ; l'écartement des lentilles est en général plus grand que la demi-somme des focales, l'achromatisme apparent n’étant réalisé que pour l'ensemble de l'oculaire terrestre.

L'intervalle entre le verre redresseur et le verre de champ, rapporté à celui des verres véhiculaires, est d'cnviron $\mathbf{I}, 3$ à $\mathbf{I}, 5$.

146. Modifications apportées à l'oculairo terrestre. - L'oculaire de Dollond ne donne, principalement en raison de la courbure de son champ, qu'un champ apparent de 0,50 au maxinum; il faut davantage quand on veut accroître l'étendue du champ réel ou augmenter le grossissement.

On l'a modifié depuis longtemps en composant les lentilles qui sont les plus éloignées des images qu'elles transforment. En remplaçant le redresseur par une lentille double, flint el crown collés, on réduit la courbure du champ, et on améliore les bords du champ apparent, en lui laissant l'étendue de o,5o. En faisant subir, en outre, la même transformation au verre d'wil, le champ apparent pent être porté à $0 ; 7^{\circ}$, et même a 0,7 j.

Plus récemment, le commandant Daubresse a réalisé un oculaire à cinq lentilles simples en crow $n$, qui donne un champ apparent de r. Il lui a suffi de disposer, en avant et près du verre d'óil, une lentille biconvexe dont 
la face antérieure est un peu plus aplatie que l'autre.

Enfin, pour certaines pelites lunettes à main de faible grossissement (8 au maximum) et de grand champ, l'aculaire terrestre est constitué comme un véritable microscope; le véhicule en est l'objectif.

I 17 . Véhicule prismatique de Porro. - La réduction de longueur des Innettes terrestres, surtout de celles qui doivent être tenues à la main, a toujours vivement préoccupé les praticiens. En 1850, un major du génie piémontais, Porro, devenu opticien, intagina une combinaison des plus remarquables. A l'aide de deux prismes à réflexion totale, remplaçant d'ailleurs le véhicule, il put raccourcir l'axe géométrique de la longue-vue, tout en conservant sa longueur à l'axe optique, qu'il brisait en cing segments successivement rectangulaires.

Considérons (fig. 53) deux prismes en verle rec-

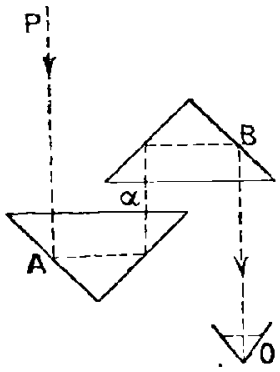

Fig. 53. tangles et isosceles A et B, dont les arêtes de l'angle droit sont parallèles, et dont les faces hypoténuses, aussi parallèles, se recouvrent par moilié. Un objet situé en $\mathbf{P}$ au-devant de la demiface apparente de $\mathbf{A}$. et dans le voisinage de la normale au nilieu d'clle, sera vu par l'ceil O placé sur la normale à la demi-face apparente de $B$. Les faisceaux incidents utiles subissent, en effet. quatre réllexions totales sur les faces de l'angle droit des prismes. L'image perçue par l'œil sera virtuelle, - c'est une image spéculaire, - non grossio et 
droite. On se rend compte de cette dernière propriété soit en construisant l'image, soif, aussi simplement, en observant que les deix prismes équivalent à quatre miroirs plans, dont l'effet de chacun d'eux est de faire subir à l'image une rotation de $90^{\circ}$ autour d'une parallèle à l'arête de l'angle droit, au total, une rotation de $360^{\circ}$.

Remarquons maintenant que, si un miroir plan tourne d'un angle $\theta$ aulour d'un rayon incident, ou d'une parallèle à celui-ci, le rayon réfléchi tourne du même angle. Si donc on fait opérer au prisme $B$ une rotation de $90^{\circ}$ autour de la normale $\alpha$ aux centres des demi-faces en regard des deux prismes, l'image perçue par l'œeil tournera, autour d'une parallèle à l'axe de rotation, d'un angle de $180^{\circ}\left(90^{\circ}\right.$ pour chacun des deux niroirs plans que constituent les faces réfléchissantes du prisme $\mathbf{B}$ ). L'image sera donc perçue renversée.

Que l'on interpose alors, en avant de A, sur le trajet du faisceau incident, un objectif convergent. Celui-ci donnera une image réelle et renversée, que le nouveau système des prismes, système croisé appelé combinaison Porro, renversera d nouveau, et maintiendra sûrement réelle, si le foyer de l'objectif se trouve an delà de la demiface de soltie de B. Ln observant cette image réelle et droitc à l'aide d'un oculaire

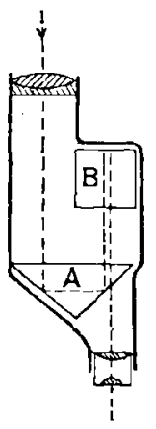

Fig. 54. positif, la lunettc-cornet de Porro sera consti tuée (fig. 54).

1 ' 8 . Lunette à prismes. - Le grand avantage de pareils instruments, - appelés aujourd'hui lunetles à prismes, - tient à la possibilité d'employer, dans 
des appareils géométriquement courts, des objectifs à Iong foyer qui penvent, par suite, être constitués simplement (à deux verres) tout en donnant des images bien nettes.

L'objectif est donc un objectif astronomique, analogue à celui des lunettes terrestres. L'oculaire sera astronomique, puisque le système des prismes remplace le véhicule (avec la seule restriction que le grossissement véhiculaire est l'unité). Il pourrait ètre d'un type quelconque; mais le verre antérieur pouvant être placé aussi voisin de l'image qu'on le désire, le type Iluygens est inutile. On lui préférera l'oculaire de Ramsden, qui donne moins de distorsion, et plus particulièrement celui à verre d'œil composé, dit achromatisé, qui conduit aisément à un champ apparent bien net de o,65 à o, zo.

Les relations géométriques entre l'objectif et l'oculaire sont celles qui conviendraient à une lunette astronomique ayant les mêmes propriétés (au renversement près de l'image). Il faut cependant tenir compte, dans les calculs, de l'allongement de focale que produit le trajet du faisceau réfracté dans les prismes, trajet égal à la somme des longueurs des hypoténuses; il est nécessaire, enfin, d'observer que le champ est physiquement limité par le phénomène de la réflexion totale, si l'on tient à avoir un champ de clarté uniforme.

L'effet du système véhiculaire prismatique est indépendant de la distance relative des prismes entre eux et à l'objectif et d̀ l'oculaire. Par un choix convenable de ces intervalles, on pourra réduire au minimum la longueur d'une lunette dont l'objectif et l'oculaire sont donnés. Le problème est du ressort de la géométrie élémentaire. Il faudra cependant observer que les 
dimensions des prismes sont d'autant plus grandes, ot que, en conséquence, la focale objective s'allonge d'autant plus que les prismes sont plus rapprochés de l'objectif.

Avec une pareille combinaison, on peut constituer des lunettes aussi peu encombrantes que celles du type de Galilée, et ayant cependant un grossissement et uxe pénétration très supérieurs, et, en outre, un champ bien plus étendu et d'ailleurs uniforménent éclairé.

I49. Dispositions diverses $d u$ véhicule Porro. - L'effet du véhicule Porro ne change pas si l'on sectionne les prismes, et si on les déplace d'une façon quelconque, pourvu que les quatre faces réfléchissantes gardent les mêmes positions relatives, et qu'en outre, pour la conservation des qualités optiques des images, les faces réfringentes d'entrée et de sortie restent normales à l'axe génćral de l'instrument. On peut disposer tout ou partie des prismes en avant de l'objectif; on pourrait même les placer en arrière de l'oculaire. La disposition la plus convenable tient au but poursuivi.

Porro avait modifié sa première combinaison pour réduire à quelques centimètres la longueur de l'appareil dans le sens de l'axe: c'est la lunette Napoléon III (14). Dans la figure 55, le système des prismes $a, b, C$ procède de celui de la figure 53 et produit les mêmes effets. La combinaison figu-

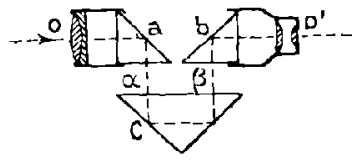

Fig. 53. rée ferait donc voir les objets renversés. Mais si l'on fait tourner de $90^{\circ}$, autour de $\alpha$, 
le prisme $a$ et l'objectif $a$, de façon à amener celui -ci en arrière du plan de la figure; et si, par une rotation de $90^{\circ}$ autour de $\beta$, on porte l'ensemble $b$ et $o^{\prime}$ vers l'avant, on obtiendra, comme précédemment, une lunette laissant les images dmites. Tinstrument sera très court suivant la normale au plạn du tableau, c'està-dire suivant la ligne visuelle; sa longueur se reportera sur la normale à l'hypoténuse du prisme $\mathrm{C}^{\prime}$, lequel sera éloigné en conséquence des deux autres, et logé dans une monture servant de poignée, verticale, à la lunette.

Quand on applique le système Porro à des lunettes munies d'objectif de grande ouverture absolne, les questions de netteté et de clarté de l'image et du prix de revient conduisent à placer les prismes dans le voisinage de l'image objective, afin que leurs dimensions restent faibles. Valgré cette sujétion, la longueur de la lunette demeure très inférieure à celle d'une longuevue ordinaire; car, dans celle-ci, l'oculaire terrestre a nne longueur qui varie des $\frac{4}{10}$ aux $\frac{7}{10}$ de la focale de l'objectif. On peut alors adopter

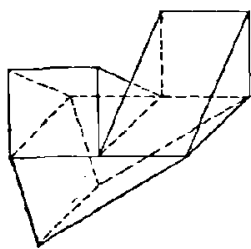

Fig. 56. pour les deux prismes, ou mieux pour les quatre demi-prismes, la combinaison la plus commode au point de vue du montage. Telle cst celle indiquée figure 56 , oì les trois éléments constituants sont collés au baume de Canada, et qui n'cst autre que la combinaison de la lunette précédente, les trois prismes étant réunis. 
Lorsqu'on désire effecturer un transport latéral d'image l'un $a$ des prismes peut être placé devant l'objectif. On obtient alors la lunette de la figure 57 , qui renverse les images. On redressera celles-ci en faisant tourner

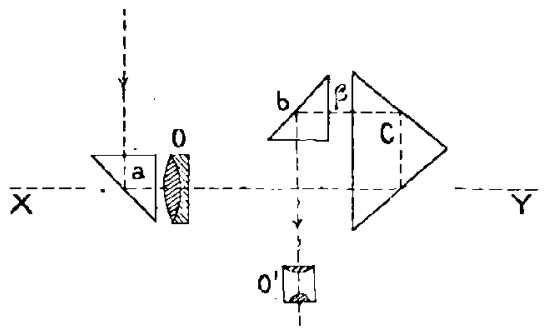

Fig. 57.

l'ensemble $\left(C b o^{\prime}\right)$ de $90^{\circ}$ autour de $x y$, puis le système $b$, $o^{\prime}$ de $90^{\circ}$ autour de la position prise par $\beta$. On obtient ainsi la combinaison employéc dans la jumellecisean do Zeiss.

I50. Véhicule tétraédrique de M. Huet. La combinaison Porro n'est pas la seule qui réalise, avec la brisure de l'axe optique, le renversement de l'image. Le problème peut ètre posé et géométriquement résolu : il s'agit, en effet, de combiner des miroirs plans de manière à atteindre le but proposé, avec cette condition que les axes des faisceaux doivent les attaquer sous l'angle de $45^{\circ}$, et cela atin de pouvoir ensuite substituer des prismes aux miroirs. Le véhicule Porro se rapporte au cas de quatre miroirs : on peut s'en fixer moins ou davantage. L'étude de la question nécessiterait des développements que nous ne pouvons introduire ici. Nous nous bornerons à signaler la solution 
élégante donnée par M. Huet ${ }^{1}$ pour le cas de six miroirs.

Considérons le système (figg. 58, A) formé de trois prismes rectangles el isoscèles dont les arêles sont perpendiculaires au tableau. Faisons tourner de $g 0^{\circ}$ le prisne $c$ autour de la normale à la face commune à $a$ et à $b$ de manière à amener la face $r$ en arrière du tableau; puis, faisons tourner le systeme total $b, c$ autour de la normale à Ia face commune à $a$ et à $b$, de
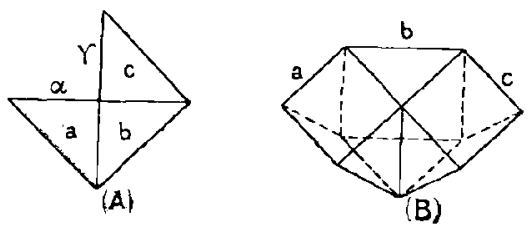

Fig. 58.

façon à amener $c$ ver's l'avant. Le système des trois prismes aura la configuration indiquée à côté (fig. 58 , B). Un faisceau entrant normalement à la face $\alpha$ sortira normalement à la face $\gamma$, qui est alors dans le même plan, après s'être réfléchie sur les faces hypolénuses de chacun des trois prismes; en outre l'image aura tourné de $90^{\circ}$ par rapport à l'axe du faisceau incillent.

Que l'on prolonge les faces réfléchissantes et réfringentes, et l'on aura un tétraèdre, solide convexe el par suite taillable dans un seul bloc de verre. Ce tétraèdre change le sens de lá direction des faisceaux et imprime à l'image une rotation d'un angle droit. L'ensemble de deux tétraèdres convenablement orientés remettra les

1 Brevet fr. 277186.

IRIS - LILLIAD - Université Lille 1 
faisceaux dans la dicection primitive en donnant à l'image une rotation de $180^{\circ}$, c'est-à-dire en la renversant : la situation relativo des tétraèdres est définie par le parallélisme des faces réfringentes et la symétrie des solides par rapport à un plan paralléle aux axes de l'objectif et de l'oculaire. Les deux tétraèdres d'une combinaison sont donc des figures symétriques, non superposables.

L'avantage de ce dispositif est de constituer des lunetles à la fois courtes, à cause du repliement de l'axe, et plates, car leur épaisseur dépend de celle d'un tétraèdre, les éléments n'y étant pas à angle droit, comme dans la combinaison Porro. La taille des tétraèdres est, cela va de soi, plus délicate et moins courante que celle des prismes isoscèles rectangles.

\section{I5I. Véhicule à prismes de Wollaston. -} Les systémes du type de Porro ou analogues opèrent le renversement de l'imagre consécutivement à son déplacement. On peut se proposer d'éviter ce dernier. II suffit d'employer deux prismes de Wollaston (fir. 59)

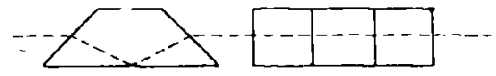

Fig. 59.

croisés ; chacun d'eux, agissant par une réflexion totale comprise entre deux réfractions, effectue une rotation de $180^{\circ}$ autour d'une droite du plan de réllexion, perpendiculaire à la ligne visuelle. En disposant les plans de réflexion à angle droit, leur intersection parallèle à l'axe de la lunette, et les deux prismes entre l'objectif et l'oculaire, le renversement demandé se pro- 
duira sans que l'image quitte l'axe primitif. Mais la solution est, sauf cas particuliers, toute thciorique : car lés prismes sont volumineux, par suite onéreux. Les pertes de lumière par réflexion et par absorption ne sont pas néggligeables, et l'instrument perd rarement de sa longueur.

1วั2. Prisme en toit. - Le prisme d'Amici ( 193 ), appelé quelquefois prisme en toit ou à toilure, a été également employé ${ }^{1}$.dans le but de transporter l'image en la redressant et en la déplaçant, au besoin, parallèlement à elle-même. Le système employé dans la jumelle Forbes ${ }^{2}$ paraît se rapporter à ce dispositif. Il n'est d'ailleur's pas le seul de cette espèce.

Ij3. Lunette de Galilée. - La combinaison optique qui constitue la lunette de Galilée est des plus

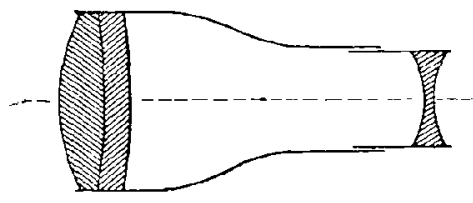

Fig. 60 . simples (fig. 6o). Un système objectif convergent donnerait, d'un objet éloigné, une image réelle et renversée; avant que celle-ci se forme, on dispose, sur le trajet du faisceau réfracté, un oculaire divergent qui rend virtuelle l'image objective, et donne en outre une image virtuelle, droite et agrandie, que l'oeil observe.

La mise au point sopère par déplacement longitudinal de l'oculaire par rapport à l'objectif. L'instrument ne comporte ni diaphragme de champ, ni diaphragmes

1 Goenz. Brevet fr. no 350557 de 1905.

g Brevet fr. 3292:6. 
de limitation : ces derniers sont constitués par les monlures des verres; mais trop ouverts pour étre, à ce point de vue, efficaces, ils laissent, en réalité, remplir leur rôle par la pupille de l'obscrvateur. L'anncau oculaire est virhel.

154. - L'objectif est généralement du type astronomique Clairaul à deux verres collés : crown biconvexe à l'extérieur, flint plan-concave à l'intéricur. L'oculaire est unc lentille simple, hiconcave, en flint; sa focale n'est pas inférieure à 3 centimètres.

L'objectif est achromatique, ou sensiblement; l'ensemble jouit approximativement de l'achromatisme apparent.

Les lunetles de cetle espèce, associćes par deux, constituent les jumelles de modèle courant, dites à six verres (trois par corps). Il existe des jumelles à 8 , io et mème I 2 verres; pour ces dernières, en particulier, les deux combinaisons de chaque lunette comportent chacune trois verres collés : à l'objectif, un flint biconcave entre deux crowns biconvexes; à l'oculaire, un crown biconvexe entre deux flints biconcaves. La faible puissance de la lunette de Galilée, quoique l'instrument soit recommandable à d'autres égards, ne justifie pas une pareille complication; le prix scul de l'instrument est augmenté; il n'est pas rare que ses qualités propres soient amoindries.

I55. Lunette hyperdioptrique. - La lunette de Galilée a un champ restreint. M. le commandant Daubresse (15) a montré qu'on pouvait y remédier en interposant une lentille convergente, dite de champ, entre l'objectif et l'oculaire. La combinaison ainsi constituée porte le nom de lunette hyperdioptrique. 
En utilisant les notations du numéro 96 , et désignant en outre par $\rho$ la focale du verre de champ, par $\lambda$ l'intervalle de celui-ci à l'objectif, la géométrie donne la relation

$$
\gamma=\frac{\Omega+G p}{\left(\mathrm{G}^{2} \varepsilon+(G-\mathrm{I}) \mathrm{F}+\mathrm{G}\left[\mathrm{G} p+\frac{\mathrm{F} p}{f}-\mathrm{F}\right]\right.} .
$$

Le champ augmentera donc si (Cf. la valeur de $\gamma$ dans $\$ 96$ ) le troisième terme du dénominatcur est négratif, les autres grandeurs restant constantes. Écrivant cette condition el tenant compte de la relation

$$
\rho=\frac{\mathrm{l} f}{\mathrm{~F}+\mathrm{f}-\boldsymbol{i}},
$$

on voit que le champ $r$, - l'ouverture de l'ohjectif, sa focale el le grossissentent restant invariables, - crô̂tra quand $f$ et $\lambda$ se rapproclieront de zéro.

Le champ de pleine lumière conserve, dans cette combinaison, le même rapport avec le champ total, que dans la lunette ordinaire : sa valeur absolue, c'est l'important, - augnente donc.

156. Appréciation des variètés de lunettes terrestres. - Pour l'observation des objets terrestres éloignés, le choix de l'opérateur peut se porter sur la longue-vué, la lunette à prismes ou la lunette de Galilée.

La lunette trrrestre, composée uniquemenl de lentilles centrées, donne, lorsqu'elle est hien construite, un champ net dans toute son étendue : la pénétration sur les bords peut ne différer qu’à peine de celle du centre. L'achromatisne peut être obtenu presque par- 
faitement. Les perles de lumière par réflexion ou par absorption restent généralement faibles. La fidélité des images est suffisante. L'amplitude du clanp, qui est uniformément éclairé, est susceptible d'atteindre une valeur $\gamma$ telle que son produit par le grossissement soit voisin de l'unité.

En présence de ces avantages, les lunettes ordinaires ont des inconvénients : elles sont longues, et par suite peu maniables; leur clarlé propre, qui dépend de la grandeur de l'anneau oculaire rarenent supérieure à a millimètres, ne peut être augnentée qu'au prix d'une exagération des dimensions devant laquelle on recule souvent. Ce sont donc des instruments qui, pendant l'observation, doivent ètre maintenus fixes; ils exigent un support. La netleté du champ dans toule son élendue permet de les utiliser dans ces conditions.

Les lunettes à prismes ont prescue les proprićtés inverses des précédentes. Moins volumineuses, elle sont mieux en main; il est possible, sans se heurter à des difficultés matérielles, de leur donner un grand anneau oculaire ( $\bar{j}$ à 6 millimètres de dianietre). Yais si leur champ peut atteindre, avec le grossissement, les mêmes valeurs que dans la longue-vue, la constance de la netteté n'est pas oblenue. En dehors de l'axe, les faisceaux traversant les prismes acquièrent de l'astigmatisme, comme dans lis traversée d'une glace épaisse à faces parallèles; les imagres perdent de leur netteté à mesure qu'on s'éloigne du centre, et on perçoit fréquemment ce défaut dans le dernicr tiers du champ. La correction de l'asligmatisme ne parait pas possible, car l'astigmation est proportionnelle à l'épaisseur des prismes dans le sens de la traversée des fais- 
ceaux. Les prismes occasionnent en outre, toujours comme une glace plane, un chromatisme latéral, qui se répercute sur l'espacement des anneaux oculaires dus aux diverses radiations : il est rare de trouver des luncttes a prismes bien achromatisées, surtout dès que le grossissement s'élève. Enlin les pertes par absorption ne sont plus aussi faibles que dans la Iunette terrestre: les prismes doivent être taillés dans une substance bien transparente; les anneaux oculaires doivent être plus ouverts.

Il résulte de ces remarques que les lunettes à prismes sont avant tout des lunettes à main; le champ efficace d'ohservation est la partie centrale du champ total, vers laguelle l'objet peut être rapidement et [resque instinctivement amené; le reste du champ n'est guère qu'un champ de recherche. Ajoutons que la netteté centrale peut être aussi bonne que dans une lunette terrestre.

En leur donnant des amneaux oculaires trùs forts, les lunettes à prismes peuvent rendre des services dans l'observation par temps sombres.

Quant à la lunette de Galilce, on a déjà énuméré ses cléfuts : faiblesse du champ, éclairement non uniforme de celui-ci, pénétration très faible. Mais elle présente un avantage indiscutable, celui de la clarté. que les autres instruments n'acquerront jamais : la supériorilé, à ce point de vue, de la lunette de Galilée se constate sans ambiguité dans l'observation courante; elle tient à son organisation sommaire, et, surtout, d̀ la possibilité pour la pupille de recevoir des faisceaux d'autant plus larges que le champ s'obscurcit davantage. En raison, en outre, de son faible volume, la lunette 
de Galilée scra par excellence l'instrument à main pour l'observation, et surtout pour la recherche, quand les objets seront faiblement éclairés (temps sombres ou brumeux, crépuscule, nuit).

\section{I57. Lunette à grossissement variable.}

Dans les lunettes bien organisécs, le grossissement et, par suite, la puissance sont inversement proporlionnels au champ. L'examen d'un objet qui demande une grande pénétration peut se contenter d'un champ restreint; mais sa recherche devient alors pénible.

On sait comment on a tourné la difficulté darns les lunetles astronomiques d'observatoire, en adjoignant à l'instrument une lunetto-chercheur à grand champ et à faible grossissement; car, dans la recherche, la pénétration ne joue qu'un róle secondaire.

La solution s'applique rarement aux lunettes terrestres : elle conduirait à doubler l'instrument. Aussi a-t-on cherché depuis longtemps à obtenir dans un mèrne appareil des grossissements différents, en rendant variable la disposition des élénents optiques constituants.

Le grossissement, rapport des focales de l'objectif et de l'oculaire, se modificra quand l'unc ou l'autre des focales, ou toutes deux, varieront. On s'adresse généralement à l'oculaire qui comporte un plus grand nombre d'éléments. Ia lunette terrestre à grossissement variable, dito polyalde dans la première moitić $\mathrm{d} u \mathrm{xr}^{\mathrm{e}}$ siècle, date de loin : Dollond en construisait sur un principe qu'on a souvent suivi. Il consiste à modifier la focale de l'oculaire en faisant varier les positions du verre redresseur et du verre de champ; car on ne saurait s'adresser à la lentille collectrice, qui doit rester près de 
l'image. Quant au verre d'oril, il suit le mouvement du champ, soit par déplacement convenable opéré par l'observateur dans la mise au point, soit par glissement automalique et réglé en conséquence, lorsqu'on tient à ce que la nuise au point soil conservée pendant toute - la variation du grossissement. Dans les appareils de ce genre, un bonton moletté déplace simultanément le verre de champ et le redresseur de leur écartement minimum, correspondant au plus faible grossissement, a leur intervalle maximum : la variation du grossissement est continue. En mème temps, le verre d'oeil, qui a été nnis préalablement au point soit par déplacement individuel, soit par glissement d'ensemble do l'oculaire terrestre entier, se porte ea s'appuyant sur un organe spécial (came, par exemple), qui le maintient à sa distance primitive de la seconde imagre réelle, laquelle suit, d’après les lois de l'optique, la déformation de l'oculaire.

On ne peut, en général, assurer pour tous les grossissements la correction des aberrations sphériques et chromatiques. Il est naturel qu'on l'opère d'abord pour le. plus fort grossissement, afin de réaliser la puissance maximum de l'instrument, et ensuite, s'il ost possible, pour le grossissement moyen, qui peut être celui du cas normal d'emploi de la lunette.

Remarquons que, puisqu'on ne touche pas à l'objectif, l'anneau oculaire grandit et que, par suite. la clarté augmente quand le grossissement diminue; sa valeur sera réglée d'après le grossissement maximum ou moyen, suivant le parti qu'on voudra tirer de l'appa reil.

La variation du grossissement peut encore être pro- 
INSTRUMLNTS D'OBSERYATION A GRATDE DISTANCE 197 duite soit par déplacement relatif du véhicule et de l'oculaire astronomique consécutif d'un glissement total de l'oculaire terrestre entier (oculaire pancratique), soit par simple mouvement de la lentille collectrice qui est alors triple.

En modifiant la focale seule de l'objectif, le grossissement change. Dans ce cas, l'objectif est constitué comme un lélé-objectif $(5)^{1}$, et les variations nécessaires sont obtenues par le déplacement de la lentille négative (divergente) : ce mouvement doit être accompagné d'un glissement automatique de tout l'oculaire ter restre. Le grossissement peut être continuellement variable, ou être seulement assuré, avec la mise au point, pour deux positions de la lentille mobile.

Les lunettes à prismes se prêtent difficilement à la variation du, grossissement : la place peut faice défiut (si l'axe est replié) à la lentille divergente; l'oculaire est trop simple pour être déformé. La solution adoptée consiste à munir l'instrument d'oculaires de forces différentes (deux en général), montés excentriquement par rappport à l'axe, et que, par un mouvement de rotation, on peut placer successivement dans le prolongement de cet axe. La mise au point n'est alors, ccla est évident, nullement assurée; elle doit être réalisée à chaque changement d'oculaire.

Quand les lunettes ne comportent que deux grossissements, l'un est souvent double de l'autre. Lorsque le grossissement est variable, on aura une idée de ses variations possibles en observant que des lunettes ont

1 La combinaison télé-ohjective a été aussi utilisée dans le but de raccourcir les lunettes astrononiques ou terrestres. (STEINherL, in Zeitsch. f. Instrk., t. XII, 1892.) 
été construites dont les grossissements variaient respectivement de 7 à 17 , de 15 à 25 , de 20 à 40 , de 60 à I50.

158. Périscopes. - Un observateur étant au pied d'une masse couvrante opaque, il s'agit pour lui de voir ce qui se passe au delà. Le problème s'est posé depuis Iongtemps au défenseur d'une place forle pour reconnaitre, en toute sécurité, les dispositions de l'assaillant. Si la solution, entrevne d'ailleurs, n'a pas été mise an point, c'est que l'accroissement aléatoire d'un danger permanent a paru moins redoutable que la complication certaine d'un appareil optique délicat.

La qucstion s'est de nouveau posće récemment, et sans espoir cette fois, qu'on pût la négliger, à propos des sous-marins : la masse couvrante, ici, est la masse d'eau en avant et au-dessus du navire; la région à explorer est la surface de la mer.

Ia solution est, dans tous les cas, la même : au dessus de la masse couvrante, un organe optique sera disposé qui recevra les faisceaux émis par les objets đu champ à observer et qui les renverra à un antre organe optique à proximité de l'observateur. Deux miroirs plans parallèles remplissent ce but : le dispositif est passé depuis longtemps dans la Physique amusante.

Un astronome hollandais, Jcan Hevel (Hévélius), indiqua ${ }^{1}$ un perfectionnement de ce tube optique, en lui adjoignant les éléments d'une lunette de Galilée. La figure $6 \mathrm{r}$, tirće des ouvrages dn $\times$ vir ${ }^{\circ}$ sic̀cle, donne une idée suffisante de cet instrument, auquel l'auteur avait donné le nom significatif de polémoscope.

- Dans la priface de sa Selenographia, sive Lune descriptio. Gedani . 1647.

IRIS - LILLIAD - Université Lille 1 
Que l'on conserve le dispositif d'Hévélius, ou qu'on hui substitue les éléments d'une lunette terrestre, l'appareil conserve un grave défaut : la limitation excessive du charnp. Il faut, en effet, remarquer que la distance entre les deux miroirs peut atteindre plusicurs mètres; les dimensions du miroir supérieur devraient donc être assez grandes, pour un champ un peu étendu. D'autre part, étant données les conditions d'utilisation de l'instrument, la substitution s'impose de prismes aux miroirs, dont il faut éviter la double image, l'urgenture sur face unique active étant à rejeter; de là l'obligation de reporter l'objectif immédiatement en avant ou en arrière du prisme supérieur, c'est-à-dire en haut de l'appareil.

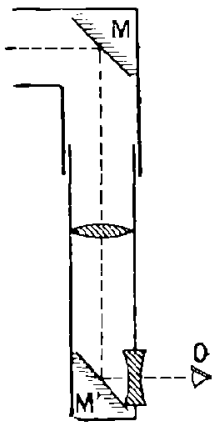

Fig. 61.

L'instrument n'est alors, aux prismes près qui produisent seulement la brisure de l'axe, qu'une lunette terrestre de très grande longucur. A cette condition absolue et non modifiable, le mode d'emploi ajoute que l'on peut se contenter d'un faible grossissement ( 4 ou 3 , et même I), mais qu'il faut le plus grand champ possible $\left(\mathrm{ro}^{\circ}\right.$ ou $\left.\mathrm{r} 5^{\circ}\right)$.

$\mathrm{Si}$, avec ces conditions, on cherche à réaliser une lunette terrestre du type cle Dollond ou du type de Galilée, un calcul sommaire d'optique géométrique montre que, pour des lunettes de 3 à 4 mètres seulement, on arrive à des ouverlures cle verre invraisemblables, et qui sont de l'ordre des gros objectifs astronomiques. La solution est à rejeter, malgré l'acuité du problème : elle est trop onéreuse. Cette consta-, 
tation paraît avoir longtemps arrêté les chercheurs.

On aurait pu cependant passer outre ${ }^{1}$. La condition gênante est relative à la grande longueur nécessaire à l'instrument. Or, la lumette terrestre de Kepler présentait ce défaut, qui est ici une qualité. La combinaison était tout indiquée.

C'est peut-ètre elle qui a été envisagée dans le cleptoscope Russo et Lauranti ${ }^{2}$ et l'hyphydroscope de Grubb ${ }^{3}$ : nous disons peut-être, parce que les textes des brevels ne font pas allusion aux difficultés surmontées. Mais on y est parvenu indirectement dans les instruments de M. Ginssberg", de M. Daubresse ${ }^{5}$, et probablement aussi dans le périscope Goerz ${ }^{6}$, en remarquant que, si dans une lunette astronomique on regarde par l'objectif, le champ devient très considérable en même temps que le grossissement s'affaiblit et descend au-dessous de l'unité. Si alors on observe cette image rapetissée avec une seconde lunette de grossissement convenable, le grossissement de l'ensemble pourra acquérir une valeur assez faible, sans que le champ diminue outre mesure, et cela en laissant aux ouvertures des verres leurs dimensions habituelles; quant à la longueur de l'instrument, elle double par l'emploi de deux lunettes.

- On youdra bien ne pas voir ici une critique de dénigrement a l'égard des inventeurs. Nous essayons de coordonner des idées et des faifs; la methode d'invention, s'il en est une, n'est pas en question. On invente comme on peut : l'essenticl cst de produire. Il n'est pas ridicule d'enfoncer une porte ouverte, si l'on est le premier à s'afercevoir qu'elle n’était pas fermíc.

2 Brevet fi. 318076 .

3 Rrevet fr. 319961 .

4 Brevet fr. 324736 .

5 Brevet fr. 370853 .

6 Brevel fr. 355349 .

IRIS - LILLIAD - Université Lille 1 
INSTRLYENTS D'OBSERVATION A GRANDE DISTANCE 201

On est donc aussi conduit à associer, objectif contre objectif, deux lunettes astronomiques de grossissements $G_{1}$ et $G_{2}$, et dont les champs récls sont $\gamma_{1}$ et $\gamma_{2}$. Le grossissement produit est $g=\frac{G_{2}}{G_{1}}$, la lunette de grossissement $G_{1}$ étant en avant. Quant au champ résultant, si les lunetles sont de même qualité $\left(\mathrm{G}_{1} \ddot{\gamma}_{1}=\mathrm{G}_{2} \gamma_{2}=h_{1}\right)$, sa valeur sera $\gamma=\mathrm{G}_{1} \gamma_{2}$ pour un grossissement total $g>$ I, qui donne $\gamma_{2}<\gamma_{1}$, ou encore

$$
\gamma=k \frac{\gamma_{2}}{\gamma_{1}}=\frac{k}{g} \text {. }
$$

En faisant $k=0,50$ ou 0,75 , valeurs possibles, on obtient $\gamma=7^{\circ}$ ou $\mathbf{1} \mathbf{I}^{\circ}$ pour $g=4$, et $\gamma=27^{\circ}$ ou $35^{\circ}$ pour $g=\mathrm{r}$.

Ces calculs approximatifs pourraient être rendus plus rigoureux en remplaçant les angles de champ par leurs tangentes, et en discutant la valeur admissible pour $k$; ils suffisent pour montrer l'ordre de grandeur des constantes de l'instrument.

rīg. Périscope et lunette de Kepler. - La combinaison optique comprenant deux lunettes astronomiques placées bout à bout est identique à celle de la lunette de Kepler-Scheiner. car il est avantageux de donner aux deux objectifs des focales égales, cette condition conduisant à la longueur maximum pour une ouverture donnée des verres et pour des qualités équivalentes de cenx-ci.

On peut étudier simplement, au point de vue actuel, la combinaison de Kepler, qui réalise le transport des images sans leur amplification. Considérons (fig. 62) les trois systèmes convergents dont les ouvertures $\mathrm{C}, \mathrm{B}, \mathbf{A}$ 
doivent être inférieures à un maximum $\Omega$ fixé d'après les conditions pratiques d'exécution.

Le grossissement de l'instrument est donné par $g=\frac{f}{f^{\prime}}$.

Pour déterminer le champ possible, formons les images des différentes surfaces actives par rapport aux systèmes anténieurs. Nous négligerons la surface arrière

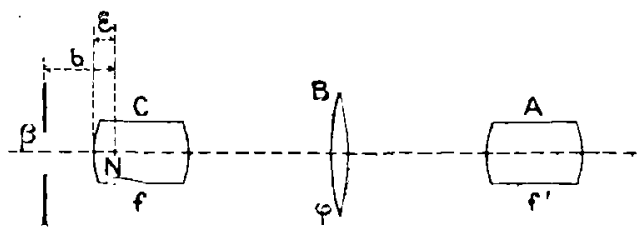

Fig. 62 .

de $\mathrm{C}$, et nous prendrons sculement les surfaces. moyennes de $B$ et de $\Lambda$; ces simplifications sont almissibles, comne le montre une discussion sommaire. On obtient sans difficulté les relations ( 1 est le nœud antérieur de $\mathrm{C}$ ) :

$$
\begin{gathered}
b=f\left[\mathrm{I}+\frac{f}{2 \varphi}\right], \quad \beta=\frac{f}{2 \varphi} \mathrm{B}, \\
a=f\left[\mathrm{x}+g\left(\mathrm{x}+\frac{f}{\rho}\right)\right], \quad \alpha=\frac{f}{f^{\prime}} \mathrm{A}^{(1)} .
\end{gathered}
$$

Les expressions possibles du champ sont alors :

$$
\pm \frac{\beta-\mathrm{C}}{b-\varepsilon}, \quad \pm \frac{\alpha-\mathrm{C}}{a-\varepsilon},
$$

(1) a, $\alpha$ sont relativement a $A$ les analogues de $b, \beta$, qui se rapportent à $B$.

IRIS - LILLIAD - Université Lille 1 
ou :

$$
\pm \frac{\frac{h}{2}-k}{1+\frac{f}{2 \rho}-\frac{f^{\prime}}{f}},(\mathrm{I}) ; \pm \frac{k^{\prime}-h}{1+g\left(1+\frac{f^{r}}{\rho}\right)-\frac{\varepsilon}{f}},
$$

$h, k, k$ étant les rapports des ouvertures des systìmes à leurs focales.

Les expressions (2) doivent être rejetées après examen sommaire, car le dénominateur renferme $g$, et, en oufre, les systemes $\mathbf{A}$ et $\mathrm{C}$ ayant des positions semblables par rapport aux images qui les concernent, on ne saurait faire $k^{\prime}$ bien différent de $k$.

Quant à l'expression (I), elle acquerra lea plus grande valeur possible quand on fera $p$ el $\equiv$ grands. Cela revient à prendre un système $B$ de grande focale, et qui peut par suite être relativement mince, et un système $\mathrm{C}$ allongé, afin que la face antéricure soit bien en avant du point nodal d'incidence. Mais alors on peut ouvrir le verre de façon que l'on ait $k>\frac{h}{2}$. $\Lambda$ lors, tant que le dénominateur reste positif, on doit prendre le signe négatif dans l'expression, et l'on voit que le système 13 travaille totalement dans toute son élendue, et l'objectif partiellement. C'est ce qui se passe daus le cas des linettes bout à bout.

On remarquera encore que, pour que la pénétration corresponde au grossissement $g$, on devra avoir, en millimètres, $\beta=2 g$. On en déduit :

$\mathrm{B}=\frac{4 g \%}{f}, \quad$ (millim),$\quad \mathrm{C}=2 g+(f-\varepsilon) \gamma+\frac{f \gamma}{2 \varphi}$.

Wn général on aura $B>C$, et ce sera à $B$ qu'on 
donnera l'ouverture maximum $\Omega$. Quant à $A$, son ouverture sera aussi, en général, inférieure à celle de $\mathrm{B}$.

On peut se demander les conditions d'un second transport, l'image définitive restant droite. Dans ce cas, réalisé géométriquenent dans la figure 63 , il sera logique de faire coïncider les ouvertures d'entrée données par $B$ et $B^{\prime}$. Il suit de là que $B$ est l'image de $B^{\prime}$ à travers $A$. En donnant a $B$ et à $B^{\prime}$ mème ouverture et même focale, écrivant que $B$ est l'image non agran-

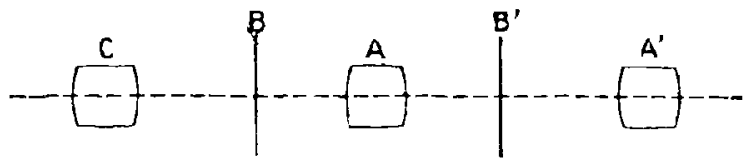

Fig. 63.

die de $B^{\prime}$ et que $A$ ne donne à l'image objective à transmettre aucun grossissement, on trouve aisément que $\mathrm{A}$ doit avoir une focale infinie. Donc $A$, système focal, est constitué par deux lentilles convergentes dont les foyers en regard coïncident. En les rendant identiques, on réalise évidemment, en raison de la symétrie, la conservation du champ primitif. Le transport de l'image se trouve assuré sur une longueur quelconque, sans autre inconvénient que la perte de clarté qui résulte des nombreux verres traversés. L'ensemble revient à plusieurs lunettes de Kepler, oculaires contre oculaires, mais avec la condition de coïncidence de leurs foyers extírieurs.

I6o. Périscope à miroir. - Il ne semble pas, au moins par ce qui a étć rendu public, que les appareils du genre précédent aient été les premiers utilisés 
dans les sous-marins. Le dispositif primitif aurait été le périscope, - le nom en a été étendu aux lunettes dont il a été question, - et qui, dû au commandant Mangin (30), consiste, en principe (fig. 64), en un miroir parabolique $M$ concave, en forme de tore donnant dans son plan focal $\Phi$ l'image.de l'horizon II. Le champ a une amplitude de $360^{\circ}$ autour de la verticale et de ro à $15^{\circ}$ de part et d'autre de l'horizon. Mais

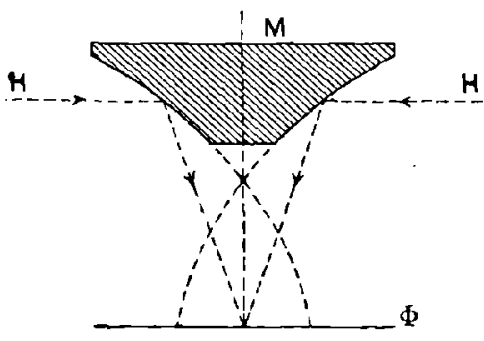

Fig. 6 4 . les images sont distordues, et peu aisément obscrvables. Pratiquement d'ailleurs, au miroir dont l'argenture serait peu résistante, a été substitué un prisme à réflexion totale, à faces courbes, également en forme de tore.

161. Lunette panoramique. - Si grand que soit le champ d'une lunette périscopique, il est nécessaire de faire tourner l'appareil sur son axe quand on veut explorer l'horizon entier. L'observateur doit suivre le mouvement. On s'est proposé d'éviter cette sujétion, en organisant l'instrument de façon que l'image définitive reste fixe dans l'espace. Dans ces conditions, l'appareil, qui devient une lunette panoramique, comporte un système objectif mobile et un système oculaire fixe. Sa forme reste coudcee, afin que les faisceaux incidents puissent atteindre l'objectif par-dessus la tète de l'observateur.

Instruments optiques. 
Si l'on se borne à faire tourner autour de l'axe vertical d'unc lunette périscopique le prisme supérieur, et l'objectif supposé en avant, tout l'horizon défile devant l'observateur; mais les images tournent en même temps autour de l'axe horizontal de l'oculaire et, en particulier, se renversent quand l'objectif regarde vers l'arrière.

La solution suivante (fig. 65) a été appliquée par la

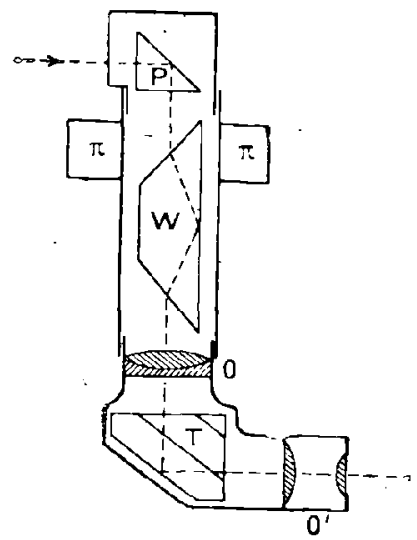

Fig. 65. maison Goerz ${ }^{1}$, pour éviter cet inconvénient. Entrel'objectif O et l'oculaire $\mathrm{O}^{\prime}$ d'une lunette astronomique est interposé un prisme à toiture $\mathrm{T}$, à réflexion presque totale; en avant de l'objectif sont disposés un prisme de Wollaston $W$ et un prismo ordinaire P. Les prismes $\mathrm{I}$ et $\mathrm{W}$ redressent l'image comme le ferait un véhicule et la placent verticalementdevant l'oculaire; les prismes $P$ el $W$ transmettent l'image à l'objectif.

Lorsque P tourne autour de l'axe vertical pour explorer l'horizon, l'image définitive reste droite, pourvu que $W$ suive le mouvement de $P$ avec une vitesse angulaire moitié moindre. Cette propriété est aisée à démontrer géométriquement. Les prismes $\mathbf{P}$ et $\mathbf{W}$ sont solidaires de pignons dentés, contenus dans une boîte $\pi$,

1 Brevet fr. 322890 .

IRIS - LILLIAD - Université Lille 1 
INSTRUMENTS D'OBSERVATION A GRANDE DISTANCE 207 qui assurent le rapport de leurs rotations. En outre, le déplacement individuel de $\mathbf{P}$ autour d'un axe horizontal normal à celui de l'appareil permet, entre certaines limites, de balayer l'espace dans un plan vertical.

Le prisme à toiture $\mathbf{T}$ peut être remplacé dans l'appareil

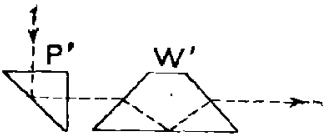

Fig. 66. précédent par un prisme ordinaire $\mathrm{P}^{\prime}$ et un autre prisme de Wollaston $\mathbf{W}^{\prime}$, tous deux fixes (fig. 66).

\section{Télescopes catoptriques.}

r62. - Le télescope dérive de la lunette astronomique ou terrestre par la substitution d'un miroir concave au système objectif. Des modifications de détail s'ensuivent quant à la constitution ou à la disposition de l'oculaire.

Les formules qui expriment le grossissement, la grandeur et la position de l'anneau oculaire, le champ réel ou apparent, seront les mèmes que pour la lunette astronomique : il suffira d'y faire entrer les focales des éléments rélléchissants. La relation qui donne la longueur matérielle de l'instrument subira, au contraire, des modifications tenant au retour des rayons qu'implique la présence de miroirs.

I63. Télescopes à vision directe. - Le premier télescope réalisé est celui de J. Gregory, avec miroir objectif annulaire et miroir véhiculaire concave. Ln siècle après, Cassegrain remplace ce dernier organe par un miroir convexe. 
Dans ces appareils, décrits dans tous les traités de Physique, la direction de la vision instrumentale est la mème qu'à l'wil nu.

I 64. Télescopes à vision latérale. -- Quelques années après Gregory, Newton conçut un télescope à miroir objectif non ouvert dans sa partie centrale.

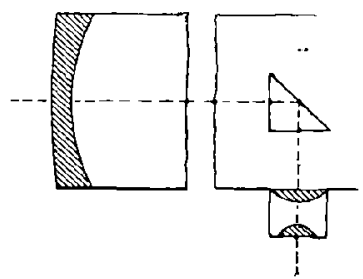

Fig. 67.

L'image produite est renvoyée latéralement $\left(\mathrm{fig}_{\mathrm{g}} \cdot 6_{7}\right)$ par un miroir plan, qui est placé à $45^{\circ}$ sur l'axe et entre le miroir concave et l'image. C'est l'image latérale réelle qui est observée à l'aide d'un oculaire astronomique. Nenton substitua d'ailleurs, dès le début, au miroir plan un prisme à réflexion totale, - un miroir de verre, comme on disait alors ${ }^{1}$, - parce que cet organe donnait une image plus parfaite que les miroirs plans. C'est le premier exemple d'utilisation des prismes à réflexion totale.

La direction de la vision est ici normale à celle de la vision directe. Les images perçues subissent, par rapport à l'objet, une rotation de $\mathbf{I} 80^{\circ}$ autour d'une perpendiculaire à la direction de la vision; elles sont par suite symétriques.

En 1786 . W. Herschel supprima, comme l'avait déjà fait Jacques Lemaire en $\mathbf{1} 728$, le miroir plan du télescope de Newton. Il se borna à recevoir l'image objective sur le bord du tube en inclinant convenable-

1 Encyclopédie méthodique. Édition Panckouke. 
ment le miroir objectif. C'est lo front-vicw telescope. Le sens de la vision est inversé relativenent à la vision directe; l'image présente le même caractère de symétrie que dans le télescope de Newton.

I65. Miroirs de télescopes. - L'idée première de la conception du télescope, - et, avant Gregrory, elle était venue à l'esprit au P. Mersenne (r6\%o) et an P. Zucchi (เ652), — était de détruire le shromatisme de l'objectif de la lunétte astronomique, en lui substituant une surface réfléchissante. Une conséquence immédiate s'ensuivait : la réduction de la longueur de l'instrument, à égale netteté d’images; car dıns la lunette l'atténuation des irisations n'était produite que grâce à des focales objectives très grandes. Même plus tard, après la découverte des objectifs achromatiques, lc tólescope conservera, au point de vue de la longueur, une légère supériorilé sur la lunette, à ouverture égale; car dans les lunettes, c'est la règle du pied pour pouce (un pied de focale par pouce d'ouverture) qui dominera. Dans les miroirs de télescope, ce rapport peut descendre pratiquement de I 2 vers 8 ou 8,5 (télescope d'Herschel, installé à Sloughi).

Jusque vers le milieu du $\mathrm{xix}^{\mathrm{v}}$ siècle, les miroirs objectifs (ct véhiculaires, s'il y a lieu) sont métalliques. L'alliage employé à leur fabrication est un bronze à environ 1 partie d'étain pour 2 de cuivre, durci par de faibles quantitís de zinc, d'argent, d'arsenic, de platine. qui le rendent plus dur et plus blanc. Chaque astro nome praticien, - car les savants s'occupent, à l'exemple d'Huygens, de la réalisation matérielle des instruments conçus, - a ses formules, variées, occasionnant parfois des déboires (mélal trop mou au polissage, ou trop 
cassant; - le mode de coulée influe autant que la composition de l'alliage). Les miroirs sont des caloltes sphériques; lord Ross, en I8/4a, s'éloignera, le premier, de cette forme dans le but de réduire les aberrations sphériques.

En I 854, L. Foucault substitıa aux miroirs métalliques des miroirs en verre; une couche d'argent, infiniment mince, était déposée sur la surface concave, puis brunie et polie. Dés lors, le miroir métallique est abandonné $e^{1}$. Ln outre, à la forme sphérique succède la forme parabolique.

Ce n'est pas au point de vne économique que se manifeste la supériorité du miroir de verre argenté sur le miroir métallique. Sans doute, le miroir n'agissant pas par réfringence, la qualité verre optique est inutile; encore faut-il que la masse soit bien homogc̀ne, afin que la taille s'effectue réguliçrement, que l'outil ne rencontre pas des régions de duretés diverses qu'il creuserait différemment. En outre, l'homogénéité doit être "suffisante pour que le verre ne travaille pas de lui-mène, ne se déforme pas, - il s'agit ici de quantités excessivement faibles. Ces conditions obligent généralement à rebuter, soit après coulće, soit après recuit, un certain nombre de disques.

Les qualités qui font rechercher le miroir en verre argenté tiennent à la perfection de sa taille, et à son pouvoir réfléchissant. Matière très dure, au sens minéralogique du mot, bien conlinue, plus homogène qu'un alliage métallique, le verre peut acquérir un poli plus

1 On y revient cependant dans les études spectrométriques, car la couche argentée est relativement transparente pour les rayons très réflangibles (ultraviolet, surtout). 
parfait que le métal, et des formes plus rapprochées des figures géométriques idéales. Les difficultés du polissage spéculaire des métaux, d'autant plus grandes que la matière est moins dure, sont bien connues des micrographes métallurgistes.

Quant au pouvoir rélléchissant, il est, relativement à un miroir métallique, bien supérieur avec un miroir en verre argenté, et se maintient plus aisément. Un miroir métallique fraîchement poli ne renvoie que les $o, 60$ de la lumière incidente; la proportion peut atteindre o,95 dans le miroir agenté, et les couleurs se conservent mieux.

Les agents atnrosphériques attaquent le miroir métallique, dont les constituants sont assez oxydables; dès que le miroir est terni, son repolissage, qui s'im pose, exige un travail d'atelier presque aussi important que le polissage primitif, et dans lequel une fausse manœuvre peut le mettre hors de service.

L'argent est moins délicat. Les émanations sulfureuses sont surtout à redouter pour lui; elles le jaunissent en augmentant fortement son pouvoir absorbant. On peut, dans les observatoires, se garantir de ces accidents. D'autre part, si, pour d'autres causes, la surface argentée se ternit légècrement, elle peut être ravivée par un polissage effectué sur place et qui no risque jamais de modifier la forme du miroir. Enfin, mème s'il y avait lieu de procéder à une réargenture, ce n'est pas celte opération qui modifierait la qualité optique du miroir.

Le verre se prête malaisément à la confection de miroirs annulaires; aussi les télescopes du genre Gregory ou Cassegrain sont-ils entièrement abandonnés. 
Le fait est sans importance. D'une utilitć contestable dans les observatoires astronomiques, ces instruments ne pouraient ctre employés, à cause de leur faible longueur, que pour les observations terrestres; mais les appareils à miroirs demandent de tels soins, que les inconvénients ccrtains dépassent les avantages probables.

I66. Télescope et lunette. - Le télescope, qui avait, dés son apparition, supplanté la lunetle astronomique, a, depuis, avantageusement luttc contre elle, malgré la découverte des systémes achromatiques.

La puissance d'une lunette ou d'un télescope, - son pouvoir résolutif des nébuleuses, par exemple, - tient d'abord à la grandeur de l'ouverture objective. A dimensions égales, lorsqu'il s'agit d'ouvertures de 5o à 8 o centimètres, de 1 mètre et même davantage, et à qualités égales, s'il se peut, Je miroir argenté demeure le plus économique; car les masses de verre optique nécessaires aux réfracteurs, - ce sont les lunettes, sont fort coùteuses.

Les télescopes sont plus courts. En donnant au miroir la forme parabolique qui détruit l'aberration sphérique pour les objets célestes, on pent réduire leur focale au triple seulement de leur ouverture.

Dans les lunetles, au conlraire, la règle ancienne du pied pour pouce ne suffit plus, toujours à qualités égales : c'est par 20 on 30 qu'il faut multiplier l'ouverture pour avoir la focale de l'objectif.

D'un autre côté, la luminosité des miroirs est très grande; on a vu plus haut que la proportion de lumière réfléchic était de o,95. Les grandes lunettes, dont les verres sont nécessairement épais, n’en transmettent guère plus de 0,65 . 
La supériorité du télescope s’accentue quand l'image. objective est reçue sur une plaque photographique. Dans le miroir, l'achromatisme est rigoureux pour toutes ces radiations. Dans l'objectif astronomique, il n'est obtenu que pour deux couleurs, car on ne peut guère songer, à moins d'augmenter les pertes de lumière, à constituer des objectifs à plus de deux verres. La netteté photographique ne s'obtient dès lors, semblable à celle que donne le miroir, qu'en allongeant la focale de l'objectif ; et l'on arrive à lui domer oo fois l'ouverture. En outre, la lumière transmise tombe à $o$, ôo et même à $o, 3 o$ : il s'agrit ici, bien entendu, de la lumière actinique, bleue, absorbée en bien plus grande quantité que les radiations jaunes ou vertes, par les crowns et les flints généralement employés dans les gros objectifs.

r67. - W. Herschel, qui a construit plus de quatre cents miroirs métalliques, al atteint, dans l'un d'eux, l'ouverture de I $/ 7$ centimètres (la focale avait i a nètres). Lord Ross a fabriqué un miroir métallique de r 83 centimc̀tres (focale de $16^{\mathrm{m}}, 8$ ), parabolique; le plus grand écart avec sa sphère osculatrice dépasse à peine 2 i..

Foucault a laissé quelques miroirs en verre argentés, d'un travail parfait. Les principaux sont ceux de I 20 centinètres (observatoire do Paris), de 83 centimètres (Toulouse) et de 80 centimètres (Marseille). La focale est d'environ six fois l'ouverture.

Le $\mathrm{D}^{\mathrm{r}}$ Common (Anglais) a construit un miroir de I5o centimètres. M. Ritchey, à l'observatoire de lerkes, en a fabriqué un de 154 centimètres d'ouverture et de $7^{\mathrm{m}}, 50$ de focale $(78)$. 


\section{Jumelles.}

I68. - Une jumelle est constituéc par deux lunettes ne renversant pas les images (lunettes terrestres, à prismes, de Galiléc), dont les axes sont parallèles et situés à l'écartement des centres des pupilles de l'observateur. Lne monlure appropriće réunit les deux corps de l'appareil; son rôle est de maintenir l'écartement, fixe ou variable, des deux lunettes, et, s'il y a lieu, de permettre leur mise au point simultanée.

En pratique, les éléments constitutifs d'une jumelle sont des lunettes à prismes ou des lunettes galiléiques. Les premières ont détrôné les longues-vues à véhicule, trop longues, dont l'emploi dans les apparcils à main n’élail guère justifié.

169. Montage des lunettes. - Dans la plupart des jumelles de théâtre ou de campagne du type Galilée, les deux lunettes sont à un écartentent invariable; la close est sans inportance tant que l'intervalle pupillaire des yeux de l'observateur ne differe de cel écartement que de I ou 2 millimètres. Mais une pareille tolérance ne peul être admise quand les lunettes (terrestres ou à prismes) comportent des anncaux oculnires róels. Dans ce cas, l'écartement des oculaires doit pouvoir prendre toutes les valeurs comprises entre deux limites données : 56 à $7^{2}$ millimètres, par exemple. Les deux corps sont alors mobiles autour d'un axe commun de rotation, ou se déplacent parallèlement gràce à un jeu de coulisses.

IRIS - LILLIAD - Université Lille 1 
INSTRUMENTS D'UBSERYATION A GRANDE DISTANGE $21 \mathrm{j}$

17o. Mise au point. - Dans les jumelles ordinaires, la mise au point s'opère par déplacement d'ensemble des deux corps, par l'intermédiaire d'un bouton moletté et d'une vis à long pas. Dans les instruments soignés, il existe, en outre, un organe correcteur qui permet de porter l'un des oculaires de part et d'autre de sa position normale, lorsqu'il y a lieu de corriger une inégalité d'accommodation des yeux. Le plus souvent, ccpendant, dans ces derniers appareils, la mise au point doit s'cxécuter séparément pour chaque corps. Ce dispositif, qui facilite la construction de la jumelle, est, à notre avis, moins commode que le précédent.

I 7 I. Jumelle stéréoscopique. - La disposition relative, dans une jumelle, de deux longues-vues ou de deux lunettes galiléiques, est unique. Il en est autrement des lunettes à prismes, au moins de celles, en majorité, qui comportent des axes différents, quoique parallèles, pour l'objectif et l'oculaire. Dans ce cas, l'écartement des objectifs peut acquérir toutes les valeurs comprises entre $\sigma+2 \eta$ et $\sigma-2 r_{1}$, $\sigma$ étant l'intervalle pupillaire, $\boldsymbol{\eta}$ le déplacement d'un axe dans une lunette; la seconde valeur peut d'ailleurs être réduite si la distance de l'axe de l'oculaire à la partie extérieure de l'appareil, comptée dans le plan des axes et perpendiculairement à cux, est inférieure à $\frac{1}{2} \varpi$; il suffit d'indiquer celte restriction.

Le cas le plus intéressant est évidemment celui, toujours réalisable, où l'écartement des objectifs est maximum. La jumelle possède alors la faculté stéréoscopique, et elle en porte la désignation. 
La combinaison Porro se prète naturellement à la disposition stéréoscopique. L'inventeur avait obtenu cette demic̀re. Si elle a reparu quarante ans plus tard comme une nouveauté, c'est que Porro avait dû abandonner et son invention el ses applications, la taille des prismes étant, à l'époque, trop onćreuse et les verres trop absorbants.

Dans la combinaison Porro, le déplacement $\eta$ d'un axe est égal à la deni-hypoténuse d'un prisme; elle permet, dans une jumelle de modèle courant, de grossissement 8 , de porter l'écartement des objectifs à $\mathrm{I}, 7^{5}$ ๘. En employant des tétraèdres de M. Huet, dans lesquels la base est, dans des conditions analogues, plus grande, cet intervalle monte à $2 \sigma$.

I 72. Jumelle à grand effet stéréoscopique. - L'écartement des objectifs peul être accru, et avec lui la faculté stéréoscopique, en augmentant $\boldsymbol{r}_{1}$, c'est-àdire Ies dimensions des prismes. La solution serait onéreuse, donnerait des instruments lourds et massifs, et conduirait à la production d'imagres très astigrmatiques. La difficulté a été surmontée dans la jumelle à grand effet stéréoscopique de Zeiss, on jumelle-ciseau, en scindant le dispositif Porro: un demi-prisme est placé à l'extérieur, remplissant le rôle d'un miroir plan renvoyant l'image à $90^{\circ}$ de sa direction; le reste de la combinaison cst transporté près du foyer de l'objectif. de façon que, ses dimensions étant faibles, l'astigmatisme soit réduit. Les images restent ainsi acceptables quelque grand que soit l'écartement des objectifs. Celui-ci, pour des modèles courants, atteint 8 , i5 et 20 fois l'intervalle pupillaire. Le grossissement individuel des luneties s'élìve aussi en sui- 
vant, ou non, la relation d'égalité indiquée par Helmholtz.

La maison Goerz remplace, dans son stéréotélescope (Br. fr. 366886, de x 9o6), la combinaison interne de l'appareil précédent par un prisme de Wollaston suivi d'un prisme en toit.

\section{${ }_{7} 7$. Appréciation des variétés de jumelles.}

- En pratique courante, le choix d'une jumelle est limité aux appareils à prismes et à la combinaison de Galilée. Il faut, en effet, mettre à part, comme instruments exceptionnels, les jumelles à deux longues-vues terrestres; ces derniers ne peuvent guère être utilisés que sur support, à moins que l'on ne se résolve à diminuer la longueur des lunettes; et cette amélioration ne peut être obtenue que par une complication des éléments optiques, qui rend l'appareil délicat et onéreux.

Entre la jumelle à prismes et la jumelle de Galilée, la décision est facile. Il suffit de se reporter à ce qui a été dit des lunettes ( 556 ). La première sera utiliscé lorsque l'observation cxigcra une certaine puissance, la perception des détails, et que la clarté sera suffisante. La jumelle de Galilée sera, avant tout, l'instrument employé dans le cas d'un éclairage médiocre des objets; sa faible puissance la désigne comme jumelle de théatre: tout en assurant une vision meilleure que l'wil nu, elle laisse encore le degré d'illusion indispensable; aussi doit-on regarder comme une superfétation les jumelles à prismes de spectacle, dont les inconvénients ne sont que trop certains (prix élevé, manque de clarté) et les avantages problématiques. D'ailleurs la jumelle de Galilée devra, avant tout, être simple : les 
iustruments a six verres sont, dans la majorité des cas, suffisants; quant au grossissement, de 2,5 à 4 en général, il sera imprudent de le porter au delà de 6 . G'est celui qui correspond à une ouverturo d'objectif de Go millimètres environ, ouverture que l'on ne peut accroître dans une jumelle, car l'intervalle des centres entro les objectifs, égal à leur diamètre augmenté de l'épaisseur de leur monturc, ne peut dépasser l'écartement pupillaire, dont la valeur moyenne est de 62 à 65 millimètres.

Pour les jumelles à prismes, le choix peut s'exercer entre les diverses combinaisons qui se différencient par le plus ou moins d'écartement des objectifs. Laissons de côté les apparcils à grand relief, nécessairement volumineux, et dont l'emploi exige un pied ou un appui, et bornons-nous aux jumelles à main.

L'accroissement d'intervalle des objectifs augmente, toutes choses égales d'ailleurs, le volume de l'instrument. Cet inconvénient est-il compensé par l'avantage que présente l'appareil d'être plus stéréoscopique? La question offre un certain intépêt.

Dans les jumelles bien maniables, le rapport maximum de l'écartement des objectifs à celui des oculaires est voisin de 2 ; le nombre 3 serait déjà un peu fort. Dans ces conditions, il n'y a pas à considérer la faculté stéréoscopique proprement dite, c'est-à-dire la possibilité d'examiner un objet sous deux points de vue différents; un simple déplacement de tête produit une variation d'aspect de l'objet au moins aussi accentuée, et avec une rapidité suffisante qui équivaut pratiquement à la simultanéité de la vision. Il ne reste donc à envisager que l'appréciation de la profondeur. 
La formule finale du 9 I22 peat mettre sous la forme

$$
\mathrm{r} m=-\frac{p}{200}\left[\mathrm{x}+\frac{p}{\sigma}\right]
$$

Apprécions la qualité d'une jumelle en évaluant la distance $p$ telle qu'une variation du dixième de sa valeur soit penceptible. La relation précédente, dans laquelle on lait $p=$ ros, donme $p=8 \mathrm{Gm}$.

Pour la jumelle habituellement ensployee dans l'artillerie de campagne, $G=8$; il en résulte que $p=150$, 250 ou 300 mètres, suivant que $m=I$ (objectifs à l'écartement des oculaires), $m=1,75$ (valeur normale) et $\boldsymbol{m}=\mathbf{2}$ (valeur maximum pratique).

Ces nombres, bien qu'on puisse en contester l'exactitude absolue, indiquent cependant qu'il ne faut pas s'cxagérer l'importance de la faculté dite stéréascopique des jumelles à main. Au delà de quelques centaines de mètres, la profondeur ne devient perceptible que pour des différences considérables.

Dès que l'observation se porte sur des objets éloignés, toute jumelle est optiquement équivalente à une lunette monoculaire. Si cependant son emploi est préférable, le fait tient à I'habitude humaine de regarder couramment avec les deux yeux; la sensation cérébrale est plus agréable, plus complète et moins fatigante; la raison est d'ordre physiologique; l'optique instrumentale n'a rien à voir dans cette question.

I74. Maniement d'une jumelle. - Quei que soit son modèle, la jumelle doit, pendant l'observation, être réglée de manière que les images se forment le plus loin possible, et que les centres des oculaires soient à l'écartement pupillaire. 
Dans les instruments à oculaires individuellement réglables, la mise au point s'effectue séparément pour chaque corps, relativement à l'wil correspondant, dans les mêmes conditions que pour une lunette.

Quand la jumelle comporte un mouvement d'ensemble des oculaires, et un système correcteur, du côtẻ droit, par exemple, la mise au point doil se faire d'abord dans la lunette gauche par déplacement simultané des oculaires, puis du côté droit sous la seule action complémentaire du dispositif correcteur.

La mise au point ayanit été ainsi rendue correcte, de préférence sur des objets éloignés, la mise à l'écartement doit s'opérer de la façon suivante. La jumelle étant complètement ouverte, de façon que l'intervalle oculaire soit supérieur à celui des pupilles, est placée devant les yeux et fermée ensuite lentement, jusqu'à ce qu'on obtienne la superposition des deux champs en un seul. Dans cette opération, l'observateur dirige la vision sur une surface uniformément éclairée, le ciel, par exemple, dans la région opposée au soleil. Il a soin de maintenir les yeux au repos, dans le vague, c'est à-dire de ne pas porter son attention sur telle ou telle région du champ, en particulier sur les bords des diaphragmes. Alors les lignes visuclles restent parallèles, et les deux corps de juniclle se trouvent disposés dans les meilleures conditions; sinon, si les yeux convergent, et comme ils tournent autour de leurs centres de rotation, l'intervalle pupillaire diminue; l'écartement des oculaires devient trop faible; et lorsqu'ensuite les images seront rejetées à l'infini, les yeux reprendront leurs positions normales, et les anncaux oculaires sortiront particllement des pupilles. 


\section{CHAPITRE X}

INSTRLMENTS D'OBSFRVATIOY A FAIBLE DISTANCE

\section{Loupe.}

I 75 . - La loupe est un système optique convergent, de focale finie et généralement faible, - quelques centimètres dans la plupart des cas, - et qui s'interpose entre l'œil et l'objet à examiner. Celui-ci est placé entre le foyer antérieur du système et la face du même côté, ou face frontale, de la loupe. 'loutes les innages produites sont virtuelles.

L'instrument est constitué par une ou plusieurs lentilles simples ou composées fixées dans une monture convenable; l'intervalle des verres est rarement variable.

- L'œil se dispose le plus près possible de la surface arrière de la loupe, la pupille centrée sur l'axe de celleci. Il faut compter entre la surface en question et la pupille apparente une distance $\boldsymbol{\eta}$, qui n'est pas inférieure à 8 millimètres. 
Le champ de la loupe s'évaluera, en tenant compte de la constitution spéciale à chaque type, comme dans les instruments à anneau oculaire virtuel; mais on ne pourra qu'imparfaitenent utiliser ce champ théorique, à cause des aberrations. Sa partie centrale, dont l'expérience déterminera l'étendue, pourra seule prétendre à constituer le véritable champ de la loupe.

Lc grossissement angulaire de la loupe est, distorsion à part, égal à l'urrité. Son grossissement, rapport des grandeurs apparentes de l'image et de l'objet, celui-ci étant placé à la elistance $\Delta_{0}$ de la vision distincte, est exprimé par

$$
\mathrm{G}=\frac{\Delta_{0}}{f},
$$

quand l'objet est situć an foyer antérieur du système, l'image étant alors reportée à l'infini.

La force de la loupe, - on l'a aussi appelée puissance, - - est l'angle sous lequel on voit l'unité de longueur de l'objet, quand l'image est amenée à la dīstance minimum $\Delta_{0}$. Son expression approchée est

$$
\frac{G_{1}}{\Delta_{\theta}} \text { ou } \frac{\mathrm{I}}{f} \text {. }
$$

176. Lonpe simple. - Ine lentille convergente simple constitue la loupe la plus vulgaire el souvent employée. La forme la plus convenable pour la correction des aberrations est le plan convexe, la face plane vers l'ouil. Le champ angulaire ne dépasse gnère 0,17 , soit une dizaine de degrés; ce sont les aberrations chromatiques qui le réluisent, l'achromatisme apparent n'étant réalisé que pour un point intérieur au système, où l'œil ne peut, par suite, se placer. 
Quand la valeur de la focale s'abaisse, que la force dè la loupe augmente, ce sont les aberrations sphériques qui interviennent pour restreindre le champ; on les réduit en diaphragmant les lentilles soit par un sillon avec (Coddington) ou sans (Wollaston) matière opaque (fig. 68, a), soit jax la forme mêmo (fig. $68, b$ ) des surfaces latérales inactives (Coddington) : il s'agit
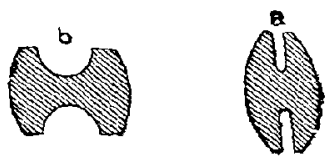

Fir. 68. ici de lentilles relativement épaisses (par rapport à leur ouverture et à leur focalc).

A la loupe simple se rattache la loupe do Stanhope (fig. 69), dans laquelle la surface antérieure plane s'appuie sur l'objet à examiner ou le reçoit (petites

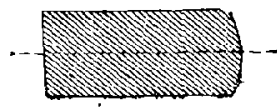

Fig. 69. photographies).

En utilisant des combinaisons achromatiques avec deux lentilles accalées, on peut porter le champ à 0,50 (soit $27^{\circ}$ ) pour des focales de so à 40 millimètres, ou à 0,35 (soit $20^{\circ}$ ) pour des focales voisines de ro millimètres. Telle est, entre autres, la loupe dite eplanélique de Steinheil (36). Avec doux lentilles sépares par un espace lenticulaire d'air, la maison Zeiss ${ }^{1}$ a indiqué une loupe (fig. 7 o) ayant un champ dépassant $30^{\circ}$ et, en outre, corrigé de la distar-

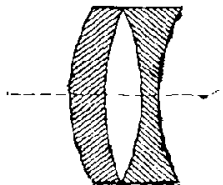

Fig. 70 . sion el de l'astigmatisme dans une large mesure.

1 Brevet fr. 338906 . 
177. Doublets; loupes composées. - L'oculaire de Ramsden et ses variantes peuvent être employés comme loupes composées. On leur préfère généralement, si l'on se borne à deux

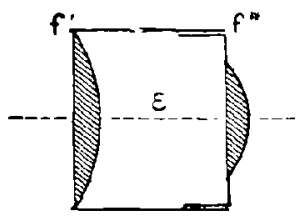

Fig. 71. lentilles simples, le doublet de Wollaston (fig. 7 I ) : c'est la loupe de mise au poinl des photographes, comprenant deux verres plan convexe ayant leurs faces planes tournées vers l'objet, avec les constantes suivantes (lentilles infiniment minces) :

$$
f=\frac{5}{3} f \quad f^{\prime}=5 f \quad \varepsilon==\frac{5}{2} f^{\prime} .
$$

La focale. de l'ensemble peul descendre jusqu'à 5 millimètres; le champ peut aller jusque vers 0,40 ou 0,50 .

D'autres combinaisons à deux verres ont été employées : deux lentilles plan convexe égales ayant les sommets de leurs faces convexes en contact (Fraunhofer) ou à une distance égale au $\frac{3}{8}$ de leur focale (Wilson) : cette dernière donnerait un champ de $40^{\circ}$.

Ch. Chevalier avait organisé une loupe sur le type d'une lunette de Galilće qui est une véritable loupe de focale infinie : une lentille frontale double et achromatique, une lentille divergente simple à l'arrière.

En composant les lentilles des doublets, en utilisant les nouveaux verres optiques, on a pu améliorer le champ de l'instrument, réaliser un achromatisme plus parfait, réduire l'astigmatisme et corriger la distorsion 
1YSTRUMENTS D'OBSERYATION A FAIBLE DISTANCE 223 si sensible dans le doublet de Wollaston. Ce sont ces propriétés qui sont revendiquées par des loupes de mise au point, telle que la loupe apodistortique de Krauss.

\section{Microscope.}

\section{$\mathrm{r}_{7} 8$. Organisation génèrale du microscope.} - Ie microscope comporte, comme la lunette astronomique, un système objectif et un système oculaire tous deux convergents, le premier donnant d'un objet une image renversée et réclle, que l'oculaire transforme en image virtuelle également renversée. Ta différence essentielle entre les deux instruments est, au point de vue optique, que la focale du microscope est finie, tandis qu'elle est infinie dans la lunette astronomique.

Le microscope d'observation ne constitue pas un instrument unique, bien défini, comme ceux que nous avons considérés jusqu'ici. Matériellement (raisons d'économie et de commodité d'emploi), il se compose, d'une part, d'une monture ou corps du microscope comprenant en particulier un tube métal lique, el, d'autre part, d'une série d'objectifs et d'une série d'oculaires.

A l'une des extrémités du tube (fig. $7^{2}$ ), dont la longucur reste, en principe, constante, se monte l'un des objectifs; à l'autre extrémité, on dispose l'oculaire. L'instrument est alors complet, et, suivant la combinaison réalisée, possède une certaine puissance. Par une association convenable des ohjectifs et des oculaires, l'opérateur peut faire varier cette puissance dans des limites étendues.

L'appareil ne comporte pas de diaphragmes de 
clarté : le mode habituel d'emploi permet d'éviter les lumières latérales. D'ailleurs, si des réflexions inté rieures se manifestept, on les évite en garnissant le tube par un cylindre en drap ou en papier noir mat; il n'en résulte aucun inconvénient pour un instrument de laboratoire.

Les montures des verres des objectifs tiennent lieu

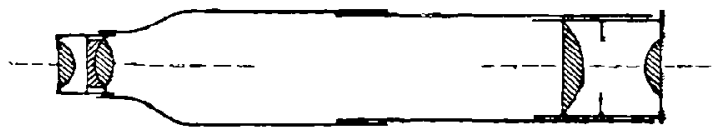

Fis. 72.

de diaphragmes de limitation; quant au diaphragme de champ, c'est généralement l'oculaire qui le porte.

La mise au point s'effectue par un déplacement d'ensemble, relativement à l'objet, du tube armé de l'objectif et de l'oculaire convenables. L'image virtuelle que l'oil observe est amenẻe, dans la mesure du possible, à la distance minimum de la vision distincte.

179. Objectif ; ouverture numérique. - Les objeclifs de microscope se classent généralemeut d'après les valeurs de leurs focales évaluées en millimètres on en pouces. Pour les moins courtes focales, l'objectif porte d'habitude un numéro d'autant plus faible que la focale est plus longue $\left(\mathrm{n}^{\mathrm{os}}\right.$ I $\grave{\mathrm{a}} 5$, dans le système français). Pour de très courtes focales, cellesci sont désignées en fraction de pouce, $\frac{\mathbf{1}}{\mathbf{1 0}}, \frac{\mathbf{1}}{\mathrm{I} \mathbf{2}}$, avec l'indication à sec, immersion à eau ou immersion homogène, suivant la nature du milieu à interposer entre l'objet et l'objectif. 
Une autre caractéristique est tout aussi utile : c'est l'ouverture numérique de l'objectif, suivant la dénomination de Abbe, qui croit habituellement en sens inverse de la focale. Un objectif L de microscope est aplanétique (fig. $7^{3}$ ) pour deux points $\mathrm{P}^{\circ}$ et $\mathrm{P}^{\prime}$ conjugués de

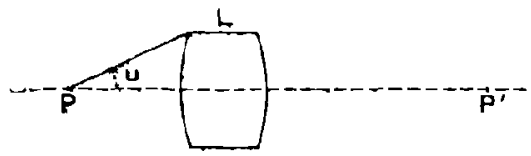

Fig. 73.

son axe. C'est en $P$ que doit être amené le point objet à observer. Le faiscean incident utile possède alors une ouverture angulaire $2 u$ qui est déterminée par la position de $\mathrm{P}$ et par l'ouverture utile de la frontale de l'objectif. Le milieu dans lequel se propago le faisceau incident utile ayant un indice $n$, l'ouverture numérique de l'objectif est définie par la relation

$$
v=n \sin u
$$

C'est cette grandeur qui est un des facteurs de la puissance d'un microscope. L'ouverture numérique varie de 0,10 d̀ 1,65 . Eulre 0,5 et 1 , elle esl dite moyenne; en dehors de ces limites, elle est faible ou grande. Au-dessous de o, ro, la puissance du microscope n'entre guère en jeu dans les observations; seule la commodité d'emploi est a considérer.

I80. Objectifs à faible ouverture. - Pour les faibles ouvertures numériques, l'objectif est employé $d \mathrm{sec}, c^{\zeta}$ est-à-dire sans interposition d'un milieu réfringent entre l'objet et la lentille frontale : objet et 
microscope plongent dans l'air. L'objectif est constitué soit par une lentille triple (fig. 74 ) formée de trois verres collés ensemble, soit par un système de deux

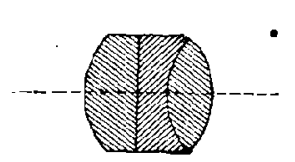

Fig. 7f.

lentilles doubles achromatiques disposées à un intervalle convenable.

La lentille triple, ulilisable jusquà l'ouverture de o, 55 , permet de réaliser l'achromatisme du système pour deux radiations, l'aplanétisme central, et de détruire la distorsion. Par un choix convenable des verres, le spectre secondaire peut être considérablement réduit.

Au-dessus de o, 5 d'ouverture numérique, l'emploi de quatre verres, réunis deux par deux, assure plus efficacement la destruction des aberrations.

I 8 I. Objectifs à moyenne ouverture. - Dans les environs de l'ouverture de 0,5 , le nombre des combinaisons doubles de l'objectif est porté à trois ; chaque -lentille double a la forme plan convexe $\left(f \mathrm{~g} \cdot 7^{5}\right)$ et com-

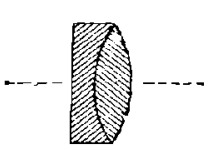

Fig. 75 .

prend un crown biconvexe et un flint plan concave, la face plane du côté de la Iumière incidente.

Quand l'ouverture augmente, on s'adresse souvent à la disposition pratiquée par Amici et qui met en curre les points aplanétiques des surfaces sphériques ou des lentilles ćpaisses. L'idée fondamentale esl de borner le rôle des lentilles antérieures à réduire l'amplitude angulaire du faisceau incident, tout en le laissant homocentrique, el cela pour la radiation moyenne; les combi naisons subséquentes assurent ensuite l'achromatisme. 
Lne lentille plan convexe épaisse $\mathrm{L}$ (fig. 76 ) possède deux points aplanétiques conjugués $\mathbf{P}$ et $\mathbf{P}^{\prime}$ séparés par son foyer antérieur $\mathrm{F}$, la facc plane recevant la lumière incidente; l'aberration sphérique, positive entre $\mathbf{P}$ et $\mathrm{P}$, est négative en dehors de ce segment. Un point lumineux placé en $\mathrm{P}$ donne une image aplanétique virtuelle en $\mathrm{P}^{\prime}$, le faisceau ayant diminué d'ouverture angulaire. Une se-

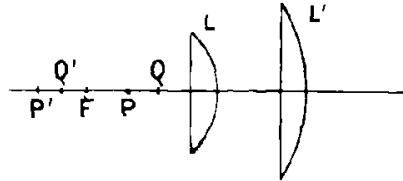

Fig. 76. conde lentille $L^{\prime}$ peut à son tour rétrécir de nouveau le faisceau en le laissant homocentrique; il suffit pour cela que son point aplanétique le plus rapproché coïncide avec $\mathrm{P}^{\prime}$.

On peut ne pas s'imposer cette sujétion et se borner à donner une partie commune aux deux scgments $P^{\prime} P^{\prime}$ et $\mathrm{QQ}^{\prime}$ limités par les points aplanétiques. Immédiatement hors de cette région commune, les deux aberrations seront de signes contraires, et la compensation de l'aberration sphérique se produira pour une position au moins de l'objet (6).

Dans les systemes à immersion homogène, - le liquide interposé a le même indice de réfraction que la lentille frontale, - on utilise (20) les points aplanétiques des surfaces sphériques (fig. 77 ). Une lentille hémisphérique $L$ de

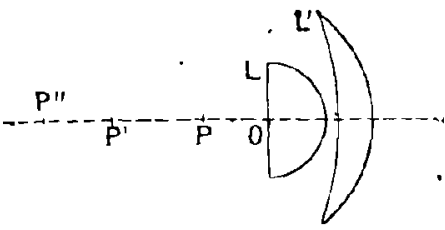

Fig. 77 .

rayon $r$ possède dans un milieu antérieur de même 
indice $n$ qu'elle, deux points aplanétiques $P$ et $P^{\prime}$, tels que

$$
\mathrm{PO}=\frac{r}{n}, \quad \mathrm{P}^{\prime} \mathrm{O}=n r .
$$

Le faisceau incident issu de $P$ sera, après réfraction, concourant en $\mathrm{P}^{\prime}$; il restera homocentrique et sera moins divergenl. Une seconde lentille $\mathbf{L}^{\prime}$ le recevra, dont la face antérieure sera centrée sur $\mathbf{P}$, face ne produisant donc aucune nouvelle réfraction, et dont la surface postérieure sera telle que P' soit l'un de ses points aplanétiques. Le faisceau diminuera de convergence, car il aura pour sommet le second point aplanétique $\mathrm{P}^{\prime \prime}$ de la surface postérieure de $\mathrm{L}^{\prime}$.

Dans les deux genres d'objec-

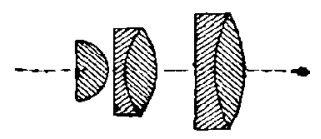

Fig. 78 . tifs, des lentilles composées reprennent ensuite le faisceau relativement fermé, l'achromatisent et le font converger de l'autre côté de l'ensemble. La figure 78 donne un exemple d'objectif d'ouverture moyenne.

182. Objectifs à immersion. - Dans les systèmes à sec, l'ouverture numérique, qui est alors égale à $\sin u$, ne peut crôitre qu'au prix de complications inadmissibles de l'objectif. En effet, $u$ augmente avec l'ouverture de la lentille frontale et le rapprochement de l'objet par rapport à celle ci. Mais la grande ouverture du faisccau nécessiterait l'emploi d'un nombre élevé de lentilles pour en diminuer successivement la divergence, car la grande ouverture absolue des verres n'est pas compatible avec une focale courte. D'ailleurs, $\sin u$ ne croît qu'assez lentement avec $u$; ainsi, en pas- 
sant de l'ouverture angulaire totale de $\mathrm{I} 20^{\circ}\left(u=60^{\circ}\right.$, $\left.\sin u=0,8_{7}\right)$ à l'ouverture limite et pratiquemment irréalisable de $180^{\circ} \quad\left(u=90^{\circ}, \sin u=\mathrm{r}\right)$, l'ouverture numérique n'augmenterait que de $15 \%$.

Il est préférable à tous égards d'agir alors sur $n$, c'est-à-dire sur le milieu interposé entre l'objet et l'objectif. En reliant par -une goutte d'eau $(n=1,336)$ l'objet à la lentille frontale, l'ouverture numérique passe de $\sin u$ à $\mathrm{r}, 336 \sin u$, augmentant aïnsi de $34 \%$ environ. Amici, en 1840, avait indiqué ce procédé de l'immersion à eau. Elle permet de porter l'ouverture numérique jusqu'à 1,20 ; avec d'autres liquides, on peul atteindre 1,60 .

Mais en accroissant ainsi l'ouverture numérique, on n'augmente le pouvoir résolvant qu'aukant que les aberrations de l'objectif diminuent en con- séquence. Le nombre de combinaisons aurmente,

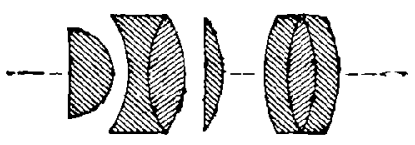

Fig. 79. leur organisation se modifie. La fignre 79 indique la constitution d'un objectif de Leitz (36), dont l'ouverture numérique (immersion homogène) est de 1,30 .

183. Objectif apochromatique. - Pour dépasser utilement cette ouverture numérique de $1,3 \mathrm{o}$, il est indispensable de réduire le spectre secondaire. On arrive alors aux combinaisons apochromatiques, dont les premières, remontant à 1886 , sont dues à Abbe. Grâce à l'emploi des nouveaux verres, qui faisaient leur apparition à cette époque, l'objectif peut être rendu aplanétique pour deux conleurs, le spectre. secondaire 
ayant disparu. La figure 80 montre la composition d'un objectif apochromatique (20). L'ouverture numérique de $\mathrm{I}, 40$ est obte-

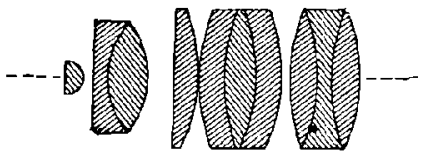

Fig. 80 . nue avec une focile de 2 millimètres.

Abbe (2) a ćgalement employé,encombinaison avec les verres opliques, le spath fluor, dont l'indice de réfraction est peu élevé ainsi que le pouvoir dispersif.

84. Oculaire. - L'oculaire du microscope est en général l'oculaire d'Iluygens avec les constantes générales indiquées pour la lunette astronomique. La combinaison préférée est celle à deux verres simples, malgré la distorsion sensible qu'elle occasionne. L'oculaire de Ramsden est peu employé, à cause de la faiblesse de son champ, dans les microscopes d'observation; il n'intervient que lorsque l'instrument est utilisé pour des mesures.

Avec les objectifs apochromatiques, lo verre de champ de l'oculaire d'Huygens est biconvexe; et si l'oculaire est fort, — c'est-à-dire si sa focale est courte. - le verre d'ceil est on outre achromatisé. Ces dispositions ont pour but de conserver tout le bénéfice réalisé par la complication de l'objectif. L'oculaire est alors dit compensateur.

I.e jeu d'oculaires d'un ensemble est en général de quatre. Le numéro s'élève à mesure que la focale diminue.

185. Emploi du microscope. - L'emploi du microscope d'observation nécessite que l'on dispose 
d'abord l'objectif et l'oculaire choisis : le premier se monte à l'extrémité inférieure du tube, le second est glissé dans la partie supérieure de celni-ci. J.e tube peul acquérir, par un tirage convenable, une longucur quelconque comprise entre certaines limites. En pratique, la puissance est maximum en même temps que la longueur du tube : objectifs et oculaires sont calculés en conséquence, et la diminution de longueur réduit le pouvoir de l'instrument à moins que l'objectif ne soit à longue correction.

Le choix des deux éléments optiques de l'instrument dépend naturellement de l'objet à examiner, et du genre d'étude entreprise. La seule règle générale à observer est de ne pas craindre de commencer par des objectifs et des oculaires faibles.

La mise au point rapide exige que l'on ait une idée assez exacte de la focale de l'objectif. Elle est amorcée par le mouvement de la crémaillère et achevée, jusqu'à ce que la netteté soit maximum, par cclui de la vis micrométrique.

186. Éclairage des objets translucides. - Le microscope est utilisé dans l'observation soit des objets partiellement perméables à la lumière, soit des surfaces des corps opaques. Le prenier cas concerne plus spécialement les physiologistes et les botanistes ; le second, Ies métallurgistes.

Dans les deux circonstances, l'éclairage de l'objet par la lumière naturelle diffuse suffit tant que le grossissement reste faible. Mais dès que la puissance de l'instrument s'élive, un ćclairage intense devient indispensable.

Les objets de la première espèce sont généralement 
constitués par des lames minces présentant dans leur structure des alternances do parties plus on moins opaques, et d'autres phus ou moins translucides. L'éclairage a lieu par transparence, c'est-à-dire que l'objet est situé entre la source lumineuse et l'objectif: les images produites sont surtout des effets de diffraction.

Pour des grossissements peu considérables, - l'anncau oculaire est alors relativement large, - on peut se borner à concentrer la lumière de la source à l'aide d'un mirair concave placé sous l'objet, ou d'un miroir plan avec lentille convergente interposée entre lui et la source. Pour des grossisscments élevés, c'est un syslème de lentilles formant condenseur qui s'intercale entre le miroir et l'objet, et qui doit avoir une ouverture au moins égale à celle de l'objectif. Le condenseur le plus simple est constitué par une demi-sphère dont la face plane regarde l'objet. Le condenseur imaginé

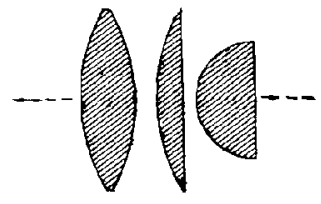

Fig. 81. par Nachet en r858, et modifié légèrement par Abbe en 1873 , comprend en outre une lentille plan-convexe et une lentille biconvexe (fig. 81). Les ouvertures sont de 1,20 , de 1,40 et de I,6o. Il existe des condenseurs achromatiques et apachromatiques, en particulier coux des constructeurs anglais Powell et Lealand $(57)$.

Le foyer du condenseur est amené sur l'objet; un diaphragme iris sert à régler, le cas échéant, l'intensité lumineuse.

187. Éclairage des objets opaques. - L'éclai- 
nge des surfaces des corps opaques est plus délicat. Il ne faut guère compter sur l'emploi d'une lentille projetant sur l'objet tes rayons obliques. Le miroir préconisé par Lieberkhun en 1748 constituerait la solution idéale, s'il porvait ètre efficace ; mais il ne convient qu'à des objectifs de grande longuenr focale.

Les divers systèmes utilisables se réduisent à deux. Au premier appartient le prisme de $\mathrm{Na}$ chet (fig. 82), prisne $\mathrm{P}$ à réflexion totale, qui peut se placer derriòre l'objectif $O$ et, par l'une des moitiés de celui-ci, transmettre sur l'objet le frisceau échairant. Le prisme est disposé dans nne monture qui suntercale entre l'objectif et le tube du microscope; un mouve-

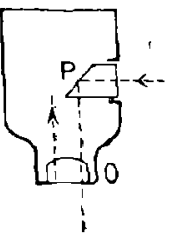

Fig. 82. ment à crémaillère permet de l'efficer, le cas échéant.

M. IH. Le Châtelier (63) a, dans son microscope de métallurgiste, modifié ce dispositif, en allongeant considérablement la partie du prisme dont la section est rectangulaire, de manière à en former une lige carrée dont la base, taillée sphériquement, forme lentille. Le tube métallique qui contient ce prisme pent receroir des diaphragmes. Un prisme à réflexion totale, ajouté par M. Charpy, permet de faire entrer dans l'appareil un faisceau provenant de sources diversement placées.

Le second système est représenté par le vertical illuminator de Smith (fig. 83), simple glace $G$ à faces parallèles placée en arrière et près de l'objectif $O$, à $45^{\circ}$ sur Jaxe. Wne ouverture

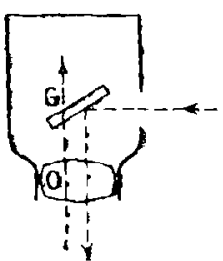

Fig. 83. du tube du micrascope laisse passer le faisceau incident. 
Cornu (fig. 84) a avantageusement remplacé ce dispositif par une pile de glaces $(x$ placée près de l'oculaire $O^{\prime}$ (11). Aucune modification

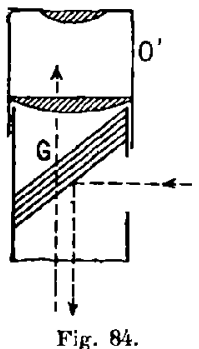
n'est ainsi apportée à l'image objective, et, en outre, la quantité de lumière envoyée à l'objet est considérablement accrue.

L'inconvénient commun à tous ces procédés tient à l'éclairement général du champ par les faisceaux rélléchis sur les diverses faces des verres d'objectif. On s'en rend compte, surtout avec les prismes, quand l'objectif est de faible numéro. Les systèmes à prismes ont en outre le défaut de ne pas laisser l'objectif travailler dans toute son ouverture : la puissance de l'instrument est nécessairement réduite. Le dispositif de Cornu se présente ainsi comme le meilleur au point de vue optique.

I 88. Sources d'éclairage. - La source ćclairante doit, suivant les circonstances, satisfaire à diverses conditions relalives à son intensité lumineuse et $\dot{a}$ la nature des radiations.

En ce qui concerne l'intensité, qui doit être d'autant plus considérable que le grossissement est plus élevé, les sources lumineuses très intenses sont, aujourd'hui, assez variées : crayon de chaux ou boule de magnésie rendus incandescents par la flamme oxhydrique (avec substitution à l'hydrogène du gaz d'éclairage, ou d'huile minérale, d'essence ou d'éther carburés), terres rares soumises à l'action du gaz d'éclairage (bec Auer) ou d'un courant électrique (lampe 
Nernst à un ou plusieurs filaments), arc électrique entre pointes de charbon ou entre métaux. La seule précaution à prendre est d'éviter la transmission des rayons calorifiques de ces sources; on les arrête en interposant entre la source et le condenseur une cuve contenant de l'eau pure ou micux de l'eau alunée. Il est prudent, en outre, d'éliminer, le cas échéant, les radiations ultra-violettes, lorsqu'elles sont nombreuses, à l'aide d'une cuve renfermant du sulfate acide de quinine, quoique les nombreux verres optiques interposés entre l'wil et la source suffisent généralement à les absorber.

Dans certains cas, l'usage du microscope demande l'emploi d'une radiation simple ou peu complexe. En particulier, la pénétration de l'instrument est caractérisée par l'intervalle des détails séparables dont l'expression est, en lumière oblique :

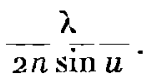

Cet intervalle décroît avec $\lambda$, et par suite, dans ces conditions, la puíssance augmente. Il y aura donc avantage à utiliser une radiation très réfrangible, qui doit cependant rester suffisamment perceptible à l'œil ; le bleu satisfait à ces conditions. On obtient cette couleur soit en la séparant par un prisme dans la lumière blanche et éliminant par des écrans les autres colorations, soit en interposant simplement entre la source et l'objet une cuve contenant du sulfate de cuivre ammoniacal, ou des verres bleus; une épaisseur liquide de quelques centimètres (2 à 5) suffit généralement.

Dans d'autres cas, il y a intérêt à employer une 
radiation simple sensible à l'ceil (jaune ou verte) : ainsi en faisant usage de la lampe à vapeur de mercure dans le vide, très intense et n'émettant qu'un pelit nombre de radialions simples, on peul ne laisser passer que la radiation verte, en absorbant le jaune par une cuve de chlorure de didyme, et les autres par une cuve de chromate de potassium. Les observations microscopiques acquièrent ainsi une douceur remarquable.

189. Objectifs à correction. - Assez fréquemment, l'observation au microscope exige que l'objet soit recouvert d'une lame transparente protectrice; c'est le cas presque constant dans l'emploi des objectifs à immersion, le liquide interposé pouvant détérioner l'objet. Ln objectif qui, sans la présence de cette lame couvre-objet, donnerait des images très nettes, perd de sa puissance quand la plaque transparente est mise en place, et cela quelle que soit la variation de mise au point qu'on opère. Ic fait avait été rcmarqué par Amici en 1829, et par lord Ross en 1837 : il est dù aux aberrations propres de la glace couvre-objet.

Les constructerurs livrent leurs objectifs corrigés pour une épaisseur donnée du couvre-objet constitué par un verre bien déterminé. Lorsqu'on veut se passer de la glace protectrice, ou en utiliser d'autres espèces, il faut avoir recours aux objectifs deorrection (les autres sont dits à monture fixe); le système inférieur de l'objectif ( $\mathrm{c}$ ou 2 lentilles) esi mobile par rapport au système supérieur (antres verres).

Si la correction est assez longue, c'est à dire le déplacement suffisamment grand, elle permet, la qualité optique étant conservée, d'employer ces objectifs avec d'autres longueurs de tube. 
Dans cortaines variétós d'objectifs, de très grande focale (par suite de faible numéro, à peu près exclusirement le $n^{\circ} \mathbf{c}$ ), le déplacement des deux parties de l'objectif n'a d'autre rôle que de faire varier focale, afin de pouvoir modifier le grossissement avec un seul organe; cette disposition a pour cause essentielle le prix assez élevé des objectifs de microscope.

r9o. Liquides d'immersioñ. - L'emploi des objectifs à immersion exige que l'on soit fixé sur la nature du liquide $\mathbf{a}$ interposer et, le cas échéant, du couvre-objet. Le but de l'immersion est de rendre $n \sin u$ le plus grand possible. En considérant dans la figure 85 , le couvre-objet d'indice $n_{1}$ et lc liquide d'indice $n_{2}$, placés devant l'ouverture MN de l'objectif, on sait que

$$
n_{1} \sin u_{1}=n_{2} \sin u_{2} \text {. }
$$

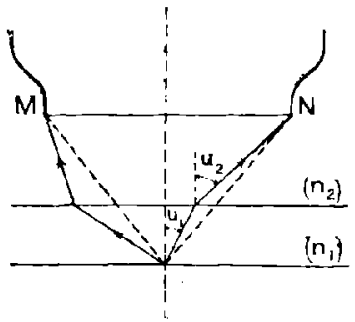

Fig. 85 .

Que l'on ait $n_{1}<n_{z}$ (côté droit de la figure), ou $n_{1}>n_{2}$ (cóté gauche), le maximum de l'ouverture numórique sera obtenu avec l'indicc commun maximum, et avec $u_{1}=u_{2}=u$. On néglige ici la variation très faible de mise au point due aux variations possibles des indices.

La première conclusion à tirer de cette remarque est l'importance de l'immersion homogène, caractérisée par l'égalité des indices du liquide et du couvre-objet; elle est róalisće depuis 1878 , suivant les indications de Abbe, en employant une glace en crown $(n=1,515)$ 
et de l'huile de cèdre pure, qui a même indice; la lentille frontale est du même crown ou d'un crown plus réfringent, afin que le faisceau n'augmente pas de divergence.

Quand la glace n'a pas exactement l'indice cidessus indiqué, on peut faire varier convenablement celui de l'huile de cèdre en la mélangeant, après l'avoir oxydée, à de l'huile d'olives.

Lorsque l'égalité des indices ne peut être obtenue, la figure montre aisément qu'il faut, dans tous les cas, réduire au minimum l'épaisscur du couvre-objet, afin de rapprocher $u_{2}$ ou $u_{2}^{\prime}$ de $u$. Dans le procéde de l'immersion à eau, indiqué par Amici en r 840, et employé en raison de sa simplicité, $n_{2}<n_{1}$.

L'immersion homogène, à l'huile de cèdre, permet de porter l'ouverture numérique jusqu'à I ,35. Au delà, les liquides employés sont le monobromure de naphtaline $(n=1,658)$, indiqué par Abbe, liquide incolore, oléagineux, à odeur de naphtaline, non volatil, mais irritant légèrement les yeux et dissolvant les résines; l'iodure de méthyle $(n=1,743)$, incolore, dense $(2,27)$, un peu volatil, à odeur d'iodoforme; l'iodure de méthyle saturé par le soufre $(n=1,787)$; la solution $(n=2,40)$ du réalgar arlificiel dans le tribromure d'arsenic, indiquée en r884 par Smith, -qui doit être employée chaude et qui s'altère rapidement (57).

C'est avec le monobromure de naphtaline qu'on a pu atteindre l'ouverture de $\mathbf{x}, 63$.

I 9 r. Placement de l'objet par rapport au microscope. - La profondeur du champ du microscope est excessivement faible, surtout avec les objectifs à courte focale. Bien que le champ rćed soit restrcint, 
il est indispensable que la surface de l'objet à exami ner, toujours à peu près plane, soit normale à l'axe de l'instrument.

Quand il s'agit d'une lame mince (coupe histologique, par exenuple), le parallélisme des faces est suffisant pour"que, la lame étant appliquée sur la platine, - bien montée dans un appareil soigné, - la face vue de l'objet soit dans les conditions indiquées. Dans d'autres cas, par exemple dans l'observation des échantillons métalliques, il serait onéreux de s'imposer la mise à épaisseur constante de l'objet, dont la face inférieure n'est pas soumise à l'examen. On peut alors utiliser le microscope coudé employé pour l'examen des réactions chimiques ou le microscope de M. Le Châtelier, qui dérive du même principe et qui comporte le système éclairant spécial pour corps opaques (63).

Danıs le mème but, nous avons fait monter un microscope ordinaire, dépourvu de son pied et de sa platine, sur le support bien connu des physiciens : deux plaques reliécs par trois ressorts de traction et maintenues à l'écartement convenable par trois vis, guidées dans la plaque supérieure et s'appuyant par leurs pointes mousses sur la plaque inférieure; une double glissière intercalée entre la plaque supérieure et le corps du microscope permet de donner à celui-ci tous les déplacements nécessaires; par le jeu des vis, on réalise l'inclinaison convenable de l'instrument, l'objet restant fixé sur son support.

192. Microscope binoculaire. - La théorie du microscope binoculaire a été donnée plus haut. La figure 86 montre deux autreß dispositifs des systèmes Instruments optiques. 
produisant le transport et la déviation des faisceaux; l'un (a) est dù à Abbe (57), l'autre à Nachet (14).
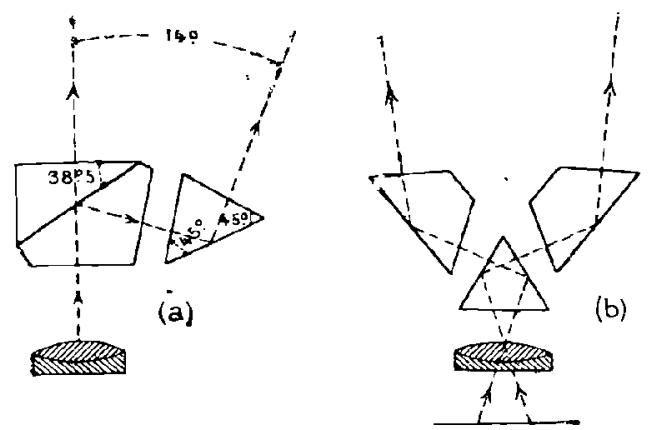

Fig. 86.

\section{Organes accessoires du microscope. -} Comme organes optiques accessoires du microscope d'obscrvation, on ne rencontre guère que les chambres claires, qui permettent de dessiner l'objet examiné, et les systèmes redresseurs d'image.

Les chambres claires sont constituées par des miroirs ou des prismes placés derrière l'oculaire (ou au-dessus de lui, suivant l'assiette de l'instrument), et qui laissent voir à la fois l'image virtuelle transportée et le papier. Il suffira ici de les signaler.

Le système redresseur a pour rôle de retourner l'image, afin de faciliter l'examen de l'ensemble. Nous indiquerons seulement le prisme rectangle d̀ bases obliques d'Amici (fig. 87), employé dans ce but par Nachet et qui a été utilisé dans d'autres appareils pour le transport des images. Les faces actives sont à 
INSTRUMENTS IOOBSERVATION A FAlBLE DISTANCE 243 angle droit en $A$, mais elles n'agissent que par réflexion partielle, les faisceaux ne tombant pas sur elles sous un angle voisin de $45^{\circ}$. Le prisme équivaut à un système de deux miroirs plans travaillant non successivement, mais simultanément, ou agissant comme un miroir plan unique normal à leur plan bissecteur et parallèle à l'arête, et qui donnerait, on outre, des images non symétriques. Les
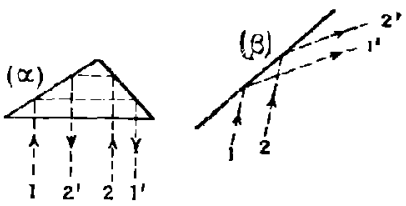
figures $\alpha$ et 3 permettent

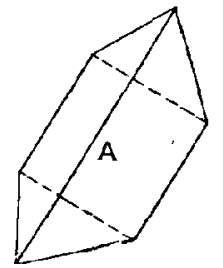

Fig. 87 de se rendre compte du mode d'action du prisme. 


\section{CIIAPITRE XI}

OBJECTIF PHOTOGRAPHIQLE ${ }^{1}$

I9. Organisation de l'objectif photographique. - L'objectif photographique est un système optique convergent, de focale finie, donnant, des objets situés au delà de son foyer antérieur, une image définitive réelle; les images intermédiaires sont virtuelles.

Les systèmes optiques utilisés en photographie et qui forment plusieurs images réelles (deux, généralement), dont la seconde seule est reçue par la plaque sensible, peuvent être considérés comme des associations d'objectifs.

Matériellement, le photo-objectif est constitué par des combinaisons centrées sur un même axe. On appelle combinaison, - il y en a une ou deux, moins souvent trois, - un ensemble de lentilles plus ou moins épaisses, accolées les unes aux autres ou séparées par de faibles intervalles; le nombre de lentilles d'une combinaison dépasse rarement trois.

Les différentes combinaisons d'ùn objectif sont

1 Pour ce chapitre, on a largement utilisé l'ouvrage (25).

IRIS - LILLIAD - Université Lille 1 
réunies, formant ainsi un système invariable en général, à une monture métallique qui porte ou reçoit les diaphragmes et qui sert également à adapter l'objectif sur la chambre noire.

Les objectifs à une ou deux combinaisons, - objeclifs simples et doubles, - ne sont munis que d'un seul diaphragme de limitation, à ouverlure variable. Cette variation peut être obtenue soit par l'agencement même de l'organe (diaphragme iris, formé de lamelles opaques articulées, dont les bords déterminent un polygone d'un grand nombre de còtés limitant l'ouverture centrale sensiblement circulaire), soit par l'introduction, dans un logement ad hoc que présente la monture, de plaques (diaphragmes à vannes) persées d'ouvertures de diamètres el, s'il y a lieu, de formes convenables.

Les triplets, - objectifs à trois combinaisons, comportent en outre un diaphragme de clarté.

Tous les objectifs sont munis de pare-soleils, souvent fixes.

195. Classification des objectifs. - L'étude des modifications subies par l'objectif photographique donne les raisons des diverses classifications dont il a été l'objet. D'ailleurs, autant de points de vue auxquels on peut se placer, autant de manières de grouper les types d'objectifs.

Le mode d'emploi ne peut guère être pris aujourd'hui comme base : il consiste dans la répartition des appareils en objectifs pour paysages, monuments, portraits, groupes, reproductions, etc. Sans atteindre d la création de l'objectif omnibus, Jes progrès de l'optique photographique ont cependant créé de larges zones 
communes enlre ces diverses calégories d'organes. Le groupement dont il s'agit ne peut ètre qu'un guide, pour le praticien, dans le choix d'un objectif étudié d'autre part.

La constitution optique offre, pour la classification, un meilleur point de départ. Elle répartit d'abord les objectifs, d'après le nombre de lcurs combinaisons, en objectifs simples, doubles et triples.

L'extension considérable prise par la seconde classe, vis-à-vis de la première et surtout de la troisième, a conduit, par la considération d'un caractère géométrique, à grouper les objectifs à deux combinaisons en doublets symétriques et asymétriques; ces derniers ont d'ailleurs tendance á supplanter les autres.

La répartition en sous-groupes est ensuite opérée d'après les qualités optiques rćalisées ou revendiquées. L'amplitude du champ a servi de guide dès l'apparition des grands angulaires, ou encore l'absence de distorsion lors de la création des reclilinéaires. Aujourd'hui ces qualités passent, au point de vue de la classification pratique, après la clarté théorique de l'objectif.

Pour l'étude qui entre dans le cadre de cet ouvrage, nous examinerons seulement, dans chacune des trois classes précitées, les diverses organisations optiques des combinaisons; un historique succinct et nécessaire en précédera l'exposé. Des indications préalables seront données relativement aux qualités optiques requises par les objectifs suivant leur mode d'utilisation.

Une dernière remarque. La plupart des objectifs portent des noms expressifs; ce n'est pas sur eux qu'il faut faire fonds pour classer ces instruments ou en 
déduire les propriétés. Même quand l'étymologie de ces expressions paraît certaine, ces marques commerciales ne renseignent pas toujours exactement sur le but poursuivi et les résultals alteints.

I96. Qualités optiques des objectifs. - La notion de finesse d'un objectif procède de la netteté de l'image; celle-ci, comme dans Ins autres instruments optiques, dépend du pouvoir séparateur, et par suite de la puissance de l'objectif. Sur l'image aérienne, la séparation possible des détails est réglée par les formules indiquées antérieurement, soit pour l'objectif astronomique, soit pour le microscope. Cette image est ici la seule à considćrer, puisqu'il n'y a pas d'organe amplificateur, tel un oculaire. Mais la plaque recevant l'image ayant des propriétés particulières, la séparation aérienne ne se conservera que dans certaines conditions sur l'image réalisée. Nous avons fait observer que la faculté séparatrice propre à la plaque sensible dépendait de sa constitution et du traitement que subissait l'image latente; c'est elle, plulôt que l'objectif, qui limitera la puissance de la chambre noire. Il est difficile, même avec des plaques au collodion, de produire deux traits fins, purs et noirs, espacés de moins de $\mathrm{O}^{\mathrm{mm}}, \mathrm{OI}$. Cetle grandeur, jointe à la connaissance des éléments optiques de l'objectif et géométriques de l'image, déterminera le pouvoir maximum d'un objectif. En général, on se tient au-dessous; car, dans la pratique courante, la séparation des détails de l'image réalisée est fixée à $\mathrm{O}^{\mathrm{mm}}, 25$ ou $\mathrm{O}^{\mathrm{mm}}, 20$ si l'image doit être observée à l'œil nu, à $o^{\mathrm{mm}}, \mathrm{I}$ ou $\mathrm{O}^{\mathrm{van}}, 05$ si clle doit être examinée à la loupe.

La clarté théorique de l'objectif est exprimée sous la 
forme $\frac{\mathbf{I}}{m}$ ou $\frac{f}{m}$. La seconde indique l'ouverture maximum utile de l'objectif, dont $f$ est la focale; dans la première, $m$ est le rapport de cette ouverture à la focale. Le nombre $m$ varic dans des limites considérables. Sa valeur normale, entièrement arbitraire d'ailleurs, a été fixće à ro: elle ne donne qu'un point de comparaison pour l'appréciation des clartés relatives de divers instruments. Les variations do $m$ s'étendent de 3 à 30 . Il a même été abaissé jusqu'à 0,5 dans des lentilles à liquides, plus curieuses que pratiques, au moins actuellement. Le jeu du diaphragme, - et alors on peut dire que l'objectif est différent, quoique restant matéricllement le même, - fait monter $m$ jusqu'à 40 .

Le champ d'un objectif est exprimé habituellement par les deux dimensions en centimètres de la plaque rectangulaire couverte, ou par l'angle correspondant à la diagonale de la plaque (ouverture angulaire du champ réel). Sa valeur, dans la plupart des objectifs, - nous en exceptons les objectifs astronomiques, - ne descend pas au-dessous de $40^{\circ}$, peut atteindre assez fréquemment 80 à $100^{\circ}$, et même arriver à $\mathrm{I} 35^{\circ}$. Ces valeurs considérables ne sont possibles que grâce au jeu du diaphragme ou à l'emploi de diaphragmes suffisamment étroits. Il est rare d'ailleurs que la netteté des bords ne soit pas sensiblement différente de celle du centre.

Le champ, sans autre spécification, doit s'entendre dans le cas des objets situés très loin (à l'infini); sa valeur augmente, pour une netteté déterminée, quand l'objet se rapproche, c'est-ì-dire quand le grossissement linéaire augmente. 
- Les objectifs dont le champ peut atteindre $7^{\circ}$ sont souvent appelés grands-angulaires ou objectifs grandangle ${ }^{1}$; vers roo $^{\circ}$ et au-dessus, ils portent le nom de panoramiques.

La distorsion n'est pas toujours parfaitement corrigée dans les objectifs, même dans ceux qui portent les noms de rectiligne, rectilinéaire, orthoscopique, et autres qualificatifs de même signification; elle est cependant alors peu perceptible, et ne devient nuisible que dans l'exécution de travaux spéciaux.

197. Caractéristiques numériques. - L'utilisation méthodique d'un objectif exige que l'on connaisse certaines caractéristiques qui peuvent être déterminées une fois pour toutes. Les principales sont : la valeur de la focale, la position des points nodaux, la situation par rapport à ccux-ci du plan du diaphragme de limitalion, et les diverses ouverlures du diaphragme (quand il s'agit d'un diaphragme iris, des repères convenables sur la bague et le corps d'objectif défnissent quelques ouvertures, les autres s'en déduisant par interpolation).

Ces renseignements devraient être indiqués sur la monture de l'objectif.

Le photographe peut, à la rigueur, et avec un peu d'habileté, déterminer ces constantes; mais il serait préfèrable qu'elles lui fussent données. Une précision de $0^{\mathrm{mm}}, 5$ est, dans la plupart des cas, largement suffisante.

En dehors de ces caractéristiques numériques ou géométriques, il serait utile que l'on connût d'avance

1 On donne aussi cette dénomination aux objectifs qui, travaillant a $f / 10$, ont un champ d'au moins $60^{\circ}$. 
le rapport entre la valeur optique (visuelle) et actinique de l'objectif.

I98. Objectif pour paysages. - L'objectif pour paysages est certainement, au point de vue numérique, le plus commun; il s'agit, dans ce cas, de la photographie d'objets inanimés à grande distance. Cette dernière expression signifie que les divers plans paraissent, du point où se trouve la chambre noire, confondus; il suffit à cet effet que le premier plan se trouve à une distance d'environ 500 fois la focale de l'objectif.

Plus exactement, en supposant l'objectif rigoureusement aplanétique et la plarue placée au foyer, la mise au point a lieu à la fois pour l'infini et pour une distance $p$ donnée par la relation :

$$
p=\frac{f^{2}}{\eta}\left(\frac{\omega}{f}\right),
$$

$\eta$ étant le ccrcle de diffusion tolérée, et $\omega$ l'ouverture de l'objectif.

En prenant :

on obtient :

$$
\frac{\omega}{f}=\frac{1}{5}, \quad \text { et } \quad \eta=0,02 \mathrm{~cm} .,
$$

$$
p=\operatorname{Iof} f^{z} \quad \text { (unité : cm.). }
$$

Pour $f=50 \mathrm{~cm}$., valeur locale assez grande, on a :

$$
p=500 f \text {. }
$$

Le cocfficient diminue d'ailleurs avec $f$ ct avec l'ouverture. Pour la focale $f==\mathrm{r} 5 \mathrm{~cm}$., l'objectif travaillant au $1 / 10$, on a :

$$
p=75 f=10 \text { mètres. }
$$

Les qualités à rechercher dans cet objectif concer- 
nent d'abord la clarté et le champ. La clarté devra prédominer dans les appareils à main et dans le cas de la photographie instantanée; le champ, dans les appareils fixes où la pose sera la règle. La valeur absolue de la focale pourra être relativement courte, car la netteté sera largement assurée; en effet, la netleté caractérisée par la valeur de $\eta_{i}$ donne le pouvoir séparateur $\alpha$, en secondes

$$
\alpha=200000 \frac{\eta}{f} \quad \text { [unité : millimètres]; }
$$

et cormme

$$
\alpha \omega=\mathrm{I} 3 \mathrm{v},
$$

il en résulte :

$$
\frac{c u}{f}=\frac{\mathrm{r} 30}{200000 r_{i}} .
$$

La valeur pratique du premier membre est toujours supérieure au second.

Quant à la distorsion, il n'y a pas généralement lieu de s'en préoccuper; și elle n'est pas très accentuée, l'aspect du paysage ne s'en ressent nullement.

Lorsque, cependant, l'objectif à paysages doit reproduire géométriquement le tableau, - le cas se présente dans les levers du terrain par la photographie (métrophotographie), - l'absence de distorsion est la qualité essenticlle. La clarté vient ensuite si l'appareil est iristantané, ou le champ si la pose peut s'effectuer.

199. Objectifs pour monuments. - Dans les objectifs pour monuments, la distorsion doit ètre inappréciable, car il s'agit alors d'ohjets ayant des formes géométriques bien caractérisées comportant de nombreuses lignes droites; l'amplitude du champ vient en scconde ligne; quant à la clarté, elle n’inter- 
vient qu'ensuite, une longue pose étant généralement possible.

La focale de l'objectif peut être courte, s'il s'agit de photographies d'objets plans (façades, par exemple); elle doit être allongée, dès qu'on veut saisir la profondeur du bâtiment.

L'emploi d'une focale longue doit encore être la règle, quand il s'agit de photographier, à faible distance, des objets ayant une certaine profondeur dans le sens de l'axe de l'objectif (petits monuments, voitures, meubles, paysages très rapprochés); sinon les déformations perspectives deviennent inadmissibles. La distorsion a ici, en général, moins d'importance; elle ne doit cependant pas être trop accentuée.

200. Objectifs pour portraits. - L'objectif pour portraits exige avant tout une clarté considérable, plus grande d'ailleurs pour les travaux d'atelier que pour la photographie en plein air. La rapidité de l'opération est ici indispensable, non seulement en raison de la fatigue qu'une pose trop longue occasionnerait au sujet, mais aussi à cause de l'attitude factice qu'elle lui imposerait. Le champ peut être faible; la netteté est généralement suffisante, d'autant plus que la retouche, nécessaire avec la nature des plaques actuelles, peut corriger bien des défauts. La focale doit ètre longue, afin que, les délails des premiers plans n'étant pas exagérés, l'image conserve une harmonie convenable. Quant à la distorsion, il est rare qu'elle soit perceptible.

Les mêmes conditions doivent être réalisées dans les objectifs pour groupes; il faut y joindre, naturellement, une amplitude du champ plus considérable. 
C'est surtout pour les portraits qu'on a recherché des objectifs de grande ouverture utile. Le problème, - dont l'intérêt, consiclérable quand on ne disposait que des plaques lentes au collodion, avait diminué à l'apparition des plaques au gélatino-bromure, s'est de nouveau posé, récemment, à propos de la photographie en couleurs par les plaques autochromes de MM. L.umière. Des objectifs travaillant à $\frac{\text { I }}{3}$ ou $\frac{\text { I }}{4}$ seraient d'une utilité incontestable.

201. Objectifs pour reproductions. - On désigne habituellement sous le nom d'objectifs pour reproductions ceux spécialement utilisés à l'agrandissement ou à la réduction (mesuré par quelques unités, I à Io au maximum) d'objets plans : dessins, cartes, documents. La fidélité des inlages est, dans ce cas, la coudition essentielle : la distorsion doit ètre absolument corrigée; le champ rendu plan sur toute l'étendue, souvent grande, de la plaque, et la netteté constante dans tout le champ. Le problème, quoique ardu, est facilité par ce fait que l'oljet est relativement près de l'objectif, et par cet autre qu'on n'a à se préoccuper qu'accessoirement de la clarté.

Ces objectifs, exclusivement destinés à la photographie industrielle, sont les plus délicats à établir, élant donné qu'on leur demande parfois de couvrir des plaques de dimensions absolues considérables $\left(\mathrm{I}^{\mathrm{m}} ; 20 \times \mathrm{I}^{\mathrm{m}}, 3 \mathrm{O}\right.$, pour reproduction en grandeur naturelle). La réalisation de pareils instruments devient encore plus pénible lorsqu'ils doivent être utilisés pour la confection des clichés destinés à la photographie par le procédé trichrome; la correction chromatique ordinaire est alors

Instruments optiques. 
insuffisante; il est indispensable d'opérer la réduction du spectre secondaire, c'est-à-dire d'avoir recours aux objectifs apochromatiques.

202. Transformations successives de l'objectif photographique. - Primitivement, l'objectif photographique est une lentille convergente quelconque. En 18I6, dans le cours de ses recherches, Niepce s'occupait surtout des lentilles dont les focales convenaient à sa chambre noire rigide. La partie centrale seule pouvait être utilisće; un diaphragme placé sur le verre couvrait les parties iuactives.

L'opticien Chevalier, qui mit en relations Daguerre et Niepce, dut leur signaler les avantages de la lentille plan-convexe et du ménisque convergent. - la face la moins courbe tournée vers l'objet, — ainsi que l'usage d'un diaphragme, placé en avant. La forme de la lentille laissait suffisant l'aplanétisme central; l'emplacement du diaphragme élargissait le champ. Ce fut le mème opticien qui fit substituer à la lentille simple un objectif achromatique de lunette astronomique.

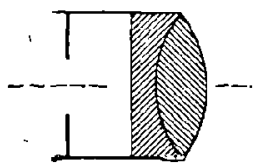

Fig. 88. Cet objectif, retournć (lo flint en avant) et muni d'un diaphragme antérieur situé au $\frac{I}{5}$ de sa focale (fig. 88), suffit, au point de vue optique, à l'achèvement des travaux de Daguerre, qui rendit son procédé public le Io août $\mathbf{r} 839$.

Ln pareil objectif ne donne qu'un champ de $28^{\circ}$, quand l'ouverture du diaphragme descend à $\frac{I}{30}$ de la focale. La clarté est insuffisante pour l'exécution 
d'un portrait. Chevalier lui substitua un objectif composé comportant (fig. 89) deux objectifs astronomiques écartés, l'ouverture du système intérieur étant la plus grande. Le diaphragme restait à l'avant.

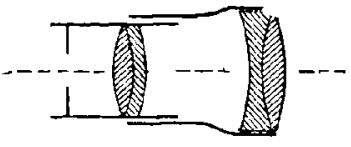

Fig. 89.

Mais, peu après, dans le courant de I84́, le professeur Petzval, de Vienne, imaginait son objectif double à portraits, qui était presque universellement adopté. L'instrument (fig. 9o) comportait deux combinaisons écartées : en avant, un objectif astronomique qui assurait l'achroma-

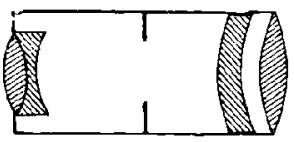

Fig. 90. tisme et l'aplanétisme suivant l'axe; à l'arrière, un ménisque divergent en flint, suivi à faible intervalle d'un verre biconvexe en crown; la seconde combinaison devait, par allongement des faisceaux, étendre le champ net en l'aplanissant et en réduisant les aberrations des pinceaux obliques. Le diaphragme, lorsqu'il était nécessaire, se disposait entre les deux combinaisons.

Pour une ouverture de $\frac{\mathrm{I}}{3,7}$, le champ pouvait 'atteindre $\mathrm{I} 5^{\circ}$; il arrivait ’̀ $30^{\circ}$ pour l'ouverture de $\frac{I}{\text { IO }}$.

Pendant trente ans au moins, l'objectif Petzval a été, pour le portrait, sans concurrent sérieux; des perfectionnements de détail lui ont été apportés dans cet intervalle et ensuite jusque vers 1880 . 
Pour l'exécution de photographies autres que les portraits, l'objectif simple à deux verres collés indiqué par Chevalier se modifiait: la forme ménisque remplaçait le plan-convexe; le crown revenait à l'extérieur, comme dans l'objectif astronomique; un troisième verre s'ajoutait aux premiers: cela dans le but de faire disparaître le foyer chimique et, à clarté égale, d'agrandir le champ de netteté. On atteignait ainsi un champ de $80^{\circ}$ en diaphragmant à $\frac{f}{40}$.

Acceptables pour les paysages, ces objectifs laissaient à désirer pour les vues de monuments, et ne convenaient que médiocrement aux reproductions: la distorsion qu'ils donnaient aux images en était la cause.

Sutton indiqua, en I 857 , le moyen de supprimer la distorsion, en constituant des objectifs symétriques; ce procédé ne fut pas immédiatement adopté. Les études étaient dirigées sur la modification du système de Petzval ; Sulton y contribuait lui-même, et elles conduisirent à la réalisation des objectifs triples ou triplets, dont le plus connu est celui de Dallemeyer, construit en $\mathrm{I} 860$.

Mais, peu d'années après, en I 866 , Dallemeyer et Steinheil réalisaient indépendamment l'objectif symétrique sous les noms respectifs de rectilinéaire et, d'aplanat.

L'objectif double, et ramassé dans le sens de son axe, - celui de Petzval est relativement long, - était constitué.

Pendant vingt ans, l'objectif ne subil plus, malgré ses innombrables variétés, que des modifications de détail. La plus importante, et qui s'est peut-être pro- 
duite en dehors d'une idée théorique, est celle qui lui fait perdre sa symétrie. Quant aux autres, elles résultent de tảtonnements, de la nécessité pour les opticiens de ne pas copier leurs concurrents, d'essais en vue de donner aux objectifs telles ou telles qualités aux dépens d'autres : elles se bornent à faire varier les formes des verres, leur nombre, leurs emplacements.

En I886, apparaissent sur le marché les nouveaux verres optiques dits verres d'Iéna (siège de la maison Schott et $\mathrm{C}^{\text {ie }}$, qui les fabrique). Ils se caractérisent d'abord par le renversement du rapport entre les indices et le pouvoir dispersif. Associós aux anciens verres, ils permettent de réaliser, sans intervention $d u$ diaphragme, la planéité du champ, concurremment avec l'achromatisme, d'après la formule indiquée depuis 1847 par Petzval. A égalité de champ net, la clarté peut ètre augmentée, sans qu'il y ait lieu d'accroître les ouvertures relatives.

En outre, le nombre des nouveaux verres s'accroît rapidement; et, si quelques déboires se sont produits soit au point de vue économique, soit au sujet du degré de conservation ou de la transparence actinique des matières, il reste encore assez de substances réfringentes pour que l'on puisse, dans les calculs d'objectifs, considérer comme une variable, et non comme une donnće, le rapport entre l'indice moyen et le pouvoir dispersif. Sans doute, cette variable n'est pas continue, comme les paramètres géométriques de système optique; mais il suffit, dans la pratique, qu'elle puisse recevoir un certain nombre de valeurs écheJonnées.

Les premiers objectifs construits avec les nouveaux 
verres ont étć lancés par la maison Zeiss, d'Iéna, en r 891 , sous le nom d'anasligmats. C'étaient des doublets non symétriques comprenant une combinaison normale et une combinaison anormale, qui étaient séparement à peu près achromatiques. L'ensemble était achromatisé avec affaiblissement du spectre secondaire; la réduction de l'astigmatisme des pinceaux obliques était obtenue par voie de différence, les deux combinaisons produisant des astigmations de signes contraires. Des triplets ont été construits peu après. L'emploi des nouveaux verres a été d'ailleurs étendu aux objectifs simples.

Il convient d'ajouter que tous les bons opticiens ont suivi l'exemple donné. Aujourd'hui, tous les objectifs de valeur comportent d'autres verres que les anciens crowns et flints; ceux-ci sont réservés aux objectifs moyens, relativernent peu coûteux, et qui peuvent, dans des cas encore assez nombreux, rendre d'excellents services.

203. Objectif simple. - L'objectif simple est constilué par une combinaison unique formée de lentilles collées ou rapprochées les unes des autres; le diaphragme est placé en avant.

L'objectif est toujours aplanétique au centre et achromatique, optiquement et actiniquement; les objectifs à foyer chimique ont actuellement disparu. La netteté des images hors du centre s'obtient souvent, aux dépens de la clarté, par le jeu du diaphragrme. Tous ces objectifs présentent de la distorsion en croissant. Cependant, en utilisant les nouveaux verres, on a pu annuler le défaut en l'un des points du champ, et alors la distorsion, faible ailleurs, a des signes con- 
traires de part et d'autre de ce point particulier. Le choix des matières permet également d'améliorer la netteté des images en réduisant l'astigmatisme.

Certains types d'objectifs doubles, symétriques ou non, sont conçus de façon qu'en retirant la combinaison antérieure, l'autre constitue un objectif simple ayant les qualités requises.

L'objectif simple est surtout un objectif à paysages. Bien que le diaphragme soit toujours étroit, dès que le champ est assez grand, la clarté peut être suffisante, surtout dans les objectifs ne comportant que des verres collés : les réflexions intérieures sont alors réduites au minimum, et la tache centrale s'évite aisément; les images restent donc brillantes. Les focales de cos objectifs doivent être relativoment grandes ; la couverture de la plaque n'est obtenue qu'à ce prix.

L'ancien objectif simple est composé de deux lentilles collées, crown et flint, celle-ci en dedans; la forme générale est celle d'un ménisque très accentué, la face concave en dehors; le diaphragme est placé au $\frac{\text { I }}{5}$ de la focale. Tel est l'objectif anglais de Grubb, de 1857 . Le champ net atteint péniblement $48^{\circ}$ à l'ouverture $-\frac{\mathrm{I}}{3 \mathrm{O}}$. Quand cette combinaison sera reprise, plus tard, en utilisant les nouveaux verres, la forme s'adoucira, se rapprochant du plan convexe dans l'objectif Voigtlander, de I 888 , travaillant à $\frac{\mathbf{I}}{\mathbf{1 5}}$ sur un champ de $9 \mathrm{o}^{\circ}$, et dans le choroscope de Goerz (fig. $9^{\mathrm{I}}$ ), où elle prendra la forme biconvexe axec une nuver- 
ture de $\frac{I}{13,5}$ et un champ de $70^{\circ}$. En même temps, le flint reviendra à l'extéricur de l'objectif, comme dans la lentille de Chevalier.

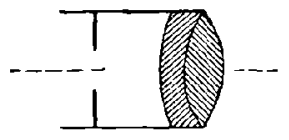

Fig. 91.

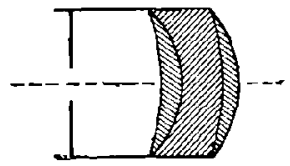

Fig. 92:

De bonne heure, une troisième lentille s'ajoute aux deux autres : un flint est collé entre deux crowns dans le grand-angulaire de Dallemeyer (fig. 92)

$$
\left(\text { (851; } y=92^{\circ} \text { pour } \omega=\frac{\mathrm{r}}{40}\right) \text {. }
$$

La forme est celle d'un ménisqué peu accentué; elle se conservera dans plusieurs objectifs du même constructeur. Plus tard, avec les nouveaux verres, ou bien la forme restera la même comme dans l'eurygraphe Berthiot ${ }^{1}$, ou se changera en celle d'une lentille biconvexe dans l'anastigmat de Zeiss de 189 /. La forme dépendra de la succession des indices : dans le premier objectif, ceux-ci vont en décroissant de l'extérieur à l'intérieur; les pouvoirs dispersifs suivent la marche inverse. Le champ restant le même, voisin de $90^{\circ}$, le diaphragme s'ouvre à $\frac{1}{14,5}$.

En se bornant à l'emploi de trois verres, il est

1 Et dans l'objectif Zeiss décrit dans le brevet fr. 374438. 
quelquefois possible d'améliorer les qualités de l'objectif en les disposant judicieusement, sans que la condition de Clairaut soit réalisée. Dallemeyer avait tenté de corriger la distorsion en retournant, dans son rectilinéaire pour vues (fig. 93), la lentille double antérieure

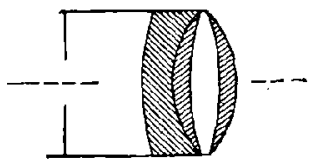

Fig. 93. de son grand-angulaire. Le champ maximum est de $50^{\circ}$ pour $\omega=\frac{\mathbf{r}}{28}$; la distorsion est alors faible.

Postérieurement, la séparation des lentilles sera décidée en suivant les idées de Gauss sur l'objectif astronomique, qui conduisent à séparer les deux éléments constituants; mais en raison des conditions spéciales à la photographie, la lentille arrière devra être composée de deux verres collés. La maison Zeiss a construit, en 1896 , un objectif simple, la lentille double arrière élant très rapprochée de l'autre; la forme générale est celle d'une lentille biconcave (fig. 94). .

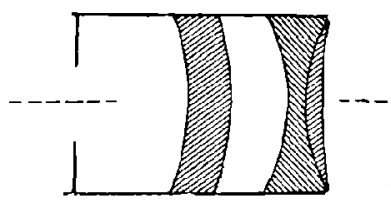

Fig. 94.

Les objectifs de cette nature comportent, en somme, quatre lentilles simples accolées, dont une d'air. On ne peut qu'y gagner, au point de vue oplique, en substituant à la lentille d'air un élément solide; il reste ensuite à examiner le côté économique. Dès $\mathrm{I} 89^{6}$, la maison Zeiss construisait des objectifs à quatre verres collés, dont deux constituaient une combinaison normale, et les autres un système anormal. Ces objectifs 
(fig. 95) très lumineux sont devenus les éléments des doublets protars; le champ de la combinaison simple est de $68^{\circ}$, avec une ouverture de $\frac{1}{\mathrm{I} 2,5}$.

Des objectifs à cinq verres collés àvaient été conçus, mais non exécutés, par Gund-

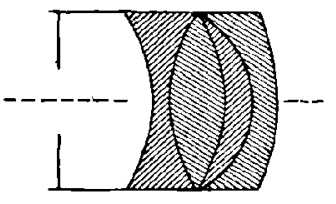

Fig. 95. lach (de Rochester). M. Lacour, constructeur français, a fait breveter ${ }^{1}$ un objectif extra-rapide et anastigmatique comprenant cinq lentilles. Par un choix convenable des matières, les trois verres antérieurs assurent l'achromatisme et l'aplanétisme central; les deux autres corrigent l'astigmatisme; la forme est celle d'un ménisque très peu accentué.

Rappelons que la plupart des systèmes arrière des objectifs symétriques peuvent être utilisés comme objectifs simples. Les combinaisons indiquées ci-dessus se rencontrent, avec des variantes, chez de nombreux opticiens; en particulier, le double anastigmat celor, de Goerz, donne un objectif simple comprenant dcux verres écartés : l'un hiconcave en avant, l'autre biconvexe à l'arrière.

204. Objectif double. - L'objectif double comporte deux combinaisons du genre des objectifs simples, séparées par le diaphragme. Il a été constitué pour la première fois par l'objectif à portraits de Petzval; le problème était alors analogue à celui qui se prósente pour un oculaire de lunette : il s'agissait

\footnotetext{
1 Brevet fr. 374045 .
} 
surtout de répartir la réfraction des faisceaux entre deux systèmes optiques.

La nécessité de corriger la distorsion conduisit au doublet symétrique. La solution paraissait évidente: on combinant deux systèmes identiques, mais symétriques par rapport au diaphragme, on devait annuler à la fois la distorsion nodale et la distorsion locale. Nous avons insisté plus haut sur ce problème.

Lo raisonnement, trop simple, avait un grave défaut : on n'en tirait pas toutes les conséquences. Puisque la disparition de la distorsion provenait uniquement de la symétrie de l'objectif, il devenait tout aussi évident qu'elle ne devait avoir lieu que pour la symétrie étendue à l'image et à l'objet : colui-ci devait donc être au double de la distance focale de l'objectif. Ln calcul rigoureux démontre l'exactitude de cette assertion. En fait, les doublets symétriques réalisaient un tel progrès sur les objectifs simples, que la distorsion semblait négligeable. Les progrès de la construction optique ont montré qu'on pouvait obtenir mieux; aussi, malgré la vogue encore persistante des combinaisons symétriques, s'adresse-t-on maintenant, pour les objectifs très soignés, aux systèmes asymétriques. Non seulement la distorsion peut être mieux corrigée, mais encore on dispose d'un nombre de paramètres presque double pour satisfaire aux conditions exigées. Le calcul des objectifs est moins simple; les qualités optiques ne peuvent $y$ perdre.

205. Doublets symétriques. - Dans les doublets symétriques, chaque combinaison est d'abord constituće par une lentille double collée, en forme de ménisque, la face convexe à l'extérieur. Telle est la 
constitution du premier objectif symétrique, le globe lens d'Harrisson, de I 860 (oủ les surfaces extérieures sont en outre sur une même sphère) (fig. 96); des pre-

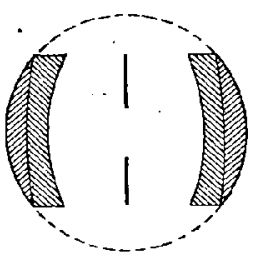

Fig. 96.

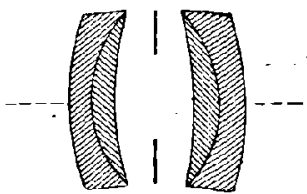

Fig. 97.

miers rectilinéaires de Dalleneyer (fig. 97) et aplanats de Steinheil, en I 866 . Cette organisation se rencontre dans un très grand nombre d'objectifs; son grand avantage est d'être simple et, par suite, peu coûteux. L'amplitude du champ n'est oblenue qu'aux dépens de la clarté, ou vice versa. Les deux combinaisons moyennement écartées, - intervalle à peu près égal à l'ouverture, - dans les objectifs dits rapides, à champ moyen (de 40 d̀ $55^{\circ}$ ), se rapprochent dans les grands-angulaires en même temps que les courbures s'accentuent et que le diaphragmo se ferme. Pour les ohjectifs à portraits, les combinaisons s'éloignent, les courbures s'aplatissent. Ces objectifs se construisent encore actuellement, et se recommandent par leurs prix modérés; c'est dans leur catégorie que se rangent la plupart des objectifs dits aplunétiques, car leur simplicité ne permet guère que la correction de l'aberration centralc. I'emploi des nouveaux verres a cependant permis une amélioration sensible des anciens 
types. A ce genre d'objectifs appartiennent, entre autres, le grand-angulaire d'Ad. Martin, les concentriques de Ross et de Schrœeder, le lynlétioscope de Goerz.

'On peut y ranger encore l'hypergone de Goerz, qui ne comporte que deux lentilles de crown, très minces, demi-sphériques, placées symétriquement par rapport au diaphragme et constituant par leur ensemble une sphère presque entière. L'objectif n'est ni achromatique ni aplanétique; la netteté des images ne s'obtient que par, le jeu du diaphragme, qui peut se fermer jusqu’à $\frac{\mathbf{I}}{3 \mathbf{I}}$; le champ atteint $\mathbf{r} 35^{\circ}$; la focale ne dépasse pas 20 centimètres.

Les objectifs dont chaque combinaison comprend trois verres collés constituent le modèle le plus courant; la forme générale de chaque système est celle d'un ménisque à bords minces ou épais, la face convexe d̀ l'extérieur. L'emploi des verres récents a permis d'accroître les qualités de nețteté et de clarté de ces instruments. C'est à eux qu'on réserve souvent, actucllement, Je qualificatif d'anastigmalique. A ce genre appartiennent certains eurygraphes de Lacour, l'orthostigmat de Stcinheil (fig.

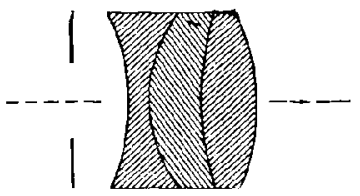

Fig. 98. $9^{8)}$, les collinéaires de Voigtlander, l'anastigmat Turillon, le double anastigmat celor de Goerz.

Pour augmenter encore la finesse des images, le nombre de lentilles de chaque combinaison a été porté 
à quatre dans l'aplanat-anastigmat de Zeiss, le double anastigmat pantar de Goerz, certains eurygraphes de Lacour, le double protar Zeiss (fig. 99), le linear de

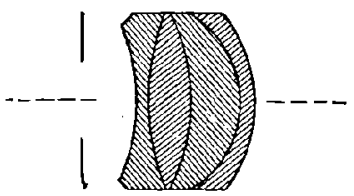

Fig. 99. Reitzchel.

On n'a pas dépassé 'le nombre de cinq lentilles collées; on rencontre de parcilles combinaisons assez onéreuses dans quelques objectifs de Lacour, dans le

double anastigmat $\frac{\mathbf{I}}{5,5}$ de Goerz.

La plupart des objectifs à trois verres par combinaison peuvent, avec des ouvertures de $\frac{\mathrm{I}}{6,5}$ à $\frac{\mathrm{I}}{\mathrm{IO}}$, avoir un champ net d'environ $65^{\circ}$. I Le jeu du diaphragme permet de porter le champ vers $90^{\circ}$. Certains objectifs sont ouverts à $\frac{\mathbf{I}}{5}$ avec un champ qui dépasse rarèment $55^{\circ}$. Quant aux objectifs comprenant un plus grand nombre de verres, aucune indication d'ordre général ne peut être donnée sur eux; ils sont conçus en vue d'un rôle bien défini.

206. - En ne s'astreignant pas au collage des verres, il est possible d'améliorer soit la clarté, soit la netteté des images; on dispose, en effet, alors d'un plus grand nombre de paramètres, courbures et intervalles des verres. La difficulté consiste alors à ne pas perdre la clarté qu'on gagne en ouvrant le diaphragme, par les réflexions sur les verres ou par la production d'une tache centrale.

Des types d'objectifs de ce genre sont le planar de 
Zeiss (fig. 100), ouvert à $\frac{\mathrm{I}}{4}$; le symétrique von Hoegh, l'unofocal de Steinheil et le lumar de Rodenstock travaillant à $\frac{\mathrm{x}}{4,5}$, le celor et le syntor de Goerz, ouverts ̀̀ $\frac{\mathbf{x}}{4,5}$ et $\frac{1}{6,8}$; l'alethar de Goerz, travaillant à

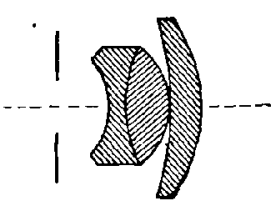

Fig. 100. $\frac{\text { I }}{\text { II }}$ avec spectre secondaire réduit.

207. Doublets asymétriques. - Le premier type - d'objectif double asymétrique se rencontre dans l'objectif à portraits de Petzval, dont la forme générale a été longtemps conservée, et dans son objectif orthoscopique, qui a été abandonné.

La symétrie primitive des rectilinćaires et des aplanats a disparu, moins à la suite de considérations théoriques que d'essais pratiques, lorsqu'on a essayé d'agrandir le champ de l'objectif. L'asymétrie manifestée dans le rectilinéaire grand-angle de Dallemeyer par la différence d'ouverture des combinaisons devient plus accentuée, par l'épaisseur des verres, dans l'antiplanat pour groupes de Stcinheil (fig. Ior). Des différences de même ordre se rencontrent dans certains objectifs anciens.

Dès l'apparition des produits d'Iéna, l'abandon de

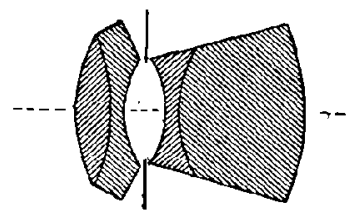

Fig. 101. la forme symétrique est corrélative de l'adoption des nouveaux verres : l'association de deux combinaisons, 
I'une normale, l'autre anormale, détruisait la symétrie optique; il n'y avait plus de raison pour conserver la symétrie géométrique. Les anastigmats de Zeiss; de I 892 , sont asymétriques.

La constitution symétrique reparaîtra quand on augmentera le nombre de verres de chaque combinaison; mais le nombre des objectifs asymétriques ira en s'accroissant rapidement soit qu'on amćliore les anciennes formes, soit qu'on en crée de nouvelles. On rencontre les dispositions les plus variées, même au seul point de vue de l'aspect géométrique. Par exemple, l'objectif de Cooke (de Taylor et Hobson), le stigmatique $\underset{-\frac{\mathrm{I}}{4}}{\mathrm{a}}$ de Dallemeyer, l'anastigmat Zeiss $\frac{\mathrm{I}}{8}$, le planigraphe Turillon, qui a la même forme que le précédent, l'unar de Zeiss, l'héliar de Voigtlander (fig. I02)

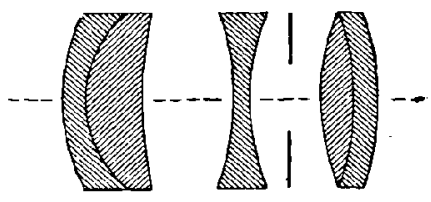

Fig. 102.

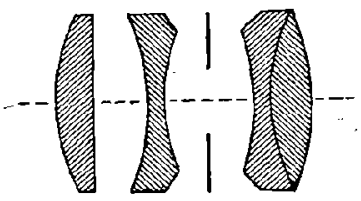

Fig. 103.

à $\frac{\mathrm{I}}{4,8^{-}}$, le kalloptat de Krauss, le tessar de Zeiss (fig. ro3). C'est dans la classe des objectifs asymétriques qu'on rencontre, le plus souvent, ceux qui réunissent au plus haut degré les qualités de netteté optique et actinique, de clarté, de champ et d'orthoscopie.

208. Triplets - La catégorie des triplets ne comprend qu'un petit nombre d'objectifs. Porro en avait 
réalisé un en $\mathrm{r} 847$. En $\mathrm{r} 859$, Sutton imagina son triosymétrique, qui, transformé, devint le triplet de Dallemeyer (fig. Io/), exécuté dans le but de supprimer la distorsion. Les doublets actuels lui sont supérieurs.

On rencontre encore un triplet bien caractérisé dans l'antiplanat pour groupes

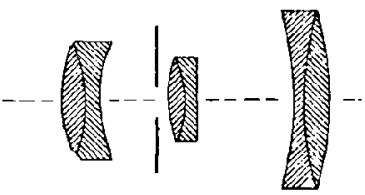

Fig. 10 '. de Steinbeil, de $\mathbf{r} 88 \mathbf{r}$; dans les triplets chromatiques et apochromatiques de Zeiss, de $189^{2}$; dans le collinéaire apochromatique de Voigtlander de i 900 . Mais entre les doublets asymétriques actuels à lentilles séparćes et les triplets, la ligne de démarcation est bien indécise.

209. Objectifs à liquides. - Pour augmenter le plus possible l'ouverture du diaphragme, el par suite la clarté des images, on a essayé d'interposer des liquides appropriés entre les verres des combinaisons ou entre celles-ci : objectif panoramique Sutton, à eau; objectif Grün ${ }^{1}$, employant les liquides des systèmes d'immersion du microscope. L'ouverture peut atteindre ainsi la valeur de la focale et mème son double. La variation des propriétés optiques des liquides avec la température, les modifications qui se produisent dans leur constitution, limitent l'usage de pareils systèmes.

2Io. Téléobjectif. - Le pouvoir séparateur de l'objectif est, dans la pratique, limité par celui de la plaque photographique. Quelle que soit la pénétration

1 Brevet fr. 318220 . 
du premier, deux objets paraîtront réunis si l'intervalle de leurs images sur le cliché descend au-dessous de $\mathrm{o}^{\mathrm{mm}}, \mathrm{o} 5$, par exemple. Pour un ensemble d'objets situé à $n$ kilomètres, l'intervalle nécessaire entre eux pour qu'ils soient discernables est, en mètres :

$$
q=\frac{50 n}{f},
$$

$f$ étant la focale de l'objectif exprimée en millimètres.

On ne peut diminuer $q$, - ou $\frac{q}{n}$, s'il agit d'un écart angulaire comme dans les observations astronomiques, - qu'en augmentant $f$. Pour la photographie céleste, $f$ a pu être porté à 60000 , comme dans la grande lunette de igoo ou le télescope réflecteur de 200 pieds de l'observatoire de Lick. Le cas est tout spécial. Pour les observations terrestres, un objectif de I mètre de focale, $f=\mathbf{I}$ ooo, est déjà encombrant; il exige cependant que les objets situés à I kilomètre soient séparés par des intervalles de $\mathbf{5}$ centimètres; cette séparation ne correspond qu'à celle d'une lunette de grossissement normal égal à 6 ou 7 . Il faut d'ailleurs parfois modifier ces limites : on ne peut compter la plaque qu'à la séparation de $o^{\mathrm{mm}}, 2$; les conditions d'emploi ne permettent pas de dépasser la focale de fo centimètres; la séparation exige alors, à y kilomètre seulement, un intervalle de $1 / 3$ de mètre. Il correspond à la vision directe, sans instrument. Le seul avantage de la photographie tient alors à la permanence de l'impression visuelle.

Cela n'est pas toujours suffisant. Les levers topographiques, les opérations de reconnaissance ou d'obser- 
vation militaires nécessitent mieux. Un objectif spécial s'impose pour la téléphotographie.

Un problème analogue, au point de vue optique, se pose dans d'autres circonstances. L'exécution d'un portrait exige un certain éloignement du sujet, dépendant de la focale de l'objectif; sinon, la déformation perspective se produit dans les divers plans en profondeur. L'image est toujours, linéairement, plus petite que l'objet, si l'on n'emploie des objectifs de très grande focale qui conduisent à des distances souvent inacceptables.

Dans tous les cas, la conclusion est la même : il faut augmenter le-grossissement linéaire de l'objectif.

2г T. Téléobjectif à transport d'image. - Le premier procédé consiste à reprendre par un autre objectif l'image aérienne du premier, qui, elle, ne subit pas l'influence de la plaque sensible. Ce second ohjectif, c'est ce qu'on nomme habitucllement un oculaire de projection, et c'est aussi lui qui, dans la microphotographie, reçoit l'image réelle de l'objectif du microscope pour la transporter, agrandie, sur la plaque sensible. Son nom tient à ce que, dans ce but spécial, il prend la place de l'oculaire du microscope; sa constitution est, à la complication près des lentilles, celle d'un véhicule, ou encore d'un objectif double, à faible foyer, à grand intervalle des combinaisons. Quand l'image aérienne est très peu étendue, on peut la transporter à l'aide d'un objectif de microscope. Quelle que soit la combinaison adoptée, l'instrument comporte toujours une longueur assez considérable, Le procédé n’est pas susceptible d'applications généralisées.

2 I2. Télóobjectif galiléique. - La caractéristique gênante, dans la photographie à grande distance, 
n'est pas la longueur focale, c'est la distance de l'objectif a la plaque sensible. Celle-ci est toujours au foyer principal; dans la plupart des objectifs vulgaires, le point nodal d'émergence, qui est la seconde extrémité de la focale, se trouve dans le voisinage des verres de l'objectif. Il suffit, évidemment, de le porter en avant du système. Un instrument qui remplit ces conditions est bien connu, et qui est en outre de faible Iongueur : la jumelle de Galilée. L'image est toutefois virtuelle et ne peut être reçuc par la plaque. Mais, puisque dans la lunette galiléique la focale est infinie, il suffira de faire varier l'intervalle des verres pour qu'elle devienne soit positive, soit négative. Dans l'un des cas, l'image sera réelle. La combinaison correspondante est celle d'un téléobjectif : grande focale, faible distance des verres entre eux et au foyer principal d'émergence.

La combinaison téléobjective se réalise en disposant en arrière d'un objectif ordinaire, généralement double et souvent symétrique, une lentille double ou triple, à verres collés, et formant système divergent. L'orthoscopique de Peluval et, même, son objectif à portraits sont une application timide de ce système. Les véritables téléobjectifs ne furent construits qu'en I 869 par Borie et 'Tournemin, et par l' $\Lambda$ méricain 'Tolles en 1870. Depuis, tous les constructeurs, Dallemeyer, Steinheil, Jarret, Zeiss, Iermagis, Goerz, etc., fabriquent des téléobjectifs basés sur ce principe.

Pour la photographie à grande distance, la combinaison peut produire la netteté à ${ }^{\mathrm{mm}}, \mathrm{r}$, avec une ouverture assez grande; la mise au point est alors automatique. En ce qui concerne la photographie à faible distance, avec large agrandissement, - portrait en gran- 
deur naturelle, par exemple, - la mise au point s'effectue par variation de focale du téléobjectif et déplacement de la glace dépolie; car, dans ce cas, les données sont habituellement le recul, - distance peu variable du sujet à la chambre, - et l'agrandissement. - Dans le téléobjectif disposé pour la photographie éloignée, l'élément divergent est, comme dans les autres cas, eutre l'objectif convergent et le foyer de celui-ci, afin qu'il ne se produise qu'une seule inage réelle. En supposant les deux systèmes réduits à des lentilles simples, de focales absolues $f$ et $f^{\prime}$, et situés à la distance $a$, la focale du téléobjectif a pour valeur:

$$
\phi=\frac{-f f^{\mu}}{f-f-a} \text {. }
$$

L'image est réelle quand $p$ est positif, c'est-à-dire quand on a :

$$
f<f^{\prime}+a \text {. }
$$

L'emploi du téléobjectif conduit à des problèmes que le défaut d'espace ne nous permet pas d'envisager ici.

213. Accessoires de l'objectif. - Miroirs et prismes. - L'image photographique réalisée est droite, quand clle est vue à travers la plaque, car celleci peut être tournée autour de son axe; vue du côté de la couche sensible, elle est, comparée à l'objet, symétrique de celui-ci, par rapport à un plan. Il y a intérêt à détruire cette symétrie, avant sensibilisation de la glace, pour certains travaux : photocollographie, photogravure, phototypographie, quand, pour des raisons de précision ou de manipulation, on ne veut 
pas s'astreindre au retournement de la pellicule qui supporte l'image.

Le renversement nécessaire s'effectue en disposant en avant ou en arrière de l'objectif une glace, ou un prisme à réflexion totale, à $45^{\circ}$ sur l'axe de la chambre. L'objet doit naturellement être reporté à $90^{\circ}$ de l'axe. • L'emploi du prisme est très onéreux : sa place scrait, à cause de l'astigmatisme, en avant de l'objectif; on perd toutefois, alors, un peu de champ, et la chose est à considérer. La glace est plus avantageuse : on en construit, à cet effet, en métal et en verre : sa place est en arrière de l'objectif. Le miroir en verre est préférable; il agit naturellement par sa face argentée et polie, placée en regard de l'objectif. Un opérateur habile peut le polir et le réargenter, au besoin; le polissage du métal est plus délicat. Ces miroirs doivent être excellents; leur prix est alors ćlevé. Quand il s'agit d'un travail occasionnel, on peut utiliser une glace du commerce, choisie et préalablement argentée; il faut la prendre assez épaisse, 6 millimètres au moins, et avoir soin de ne pas la comprimer en l'enchâssant. Si la glace n'est pas irréprochable, on peut, en diaphragmant suffisamment l'objectif, obtenir des résultats convenables.

214. Transport des images stéréoscopiques. - Dans la photographie stéréoscopique, deux objectifs à axes parallèles donnent deux images presque identiques; mais séparées. Le cliché, vu par transparence, peut être introduit dans le stéréoscope ; mais non, vu directement, du côté de la couche sensible. Les images doivent être transposées, sinon la vision serait pseudoscopique, l'image relative à l'œil droit étant vue par l'œil gauche 
et vice versa. C'est ce qui se passe quand on tire une photocopie sur papier; il est nécessaire de découper les deux images et de les disposer autrement. On peut éviter cette sujétion et les soins qu'elle entraine en produisant optiquement la transposition dans la chambre.

Parmi les systèrnes optiques employés à cet effet, nous signalerons le dispositif du commandant Daubresse', dont la figure 105 donne une idée suffisante. Les combinaisons peuvent être variées par sectionnement de prismes, déplacements relatifs des divers éléments et substitution aux prismes indiqués de miroirs ou de prismes de formes différentes.

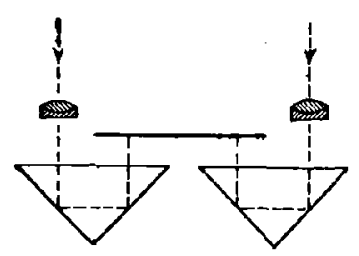

Fig. 105.

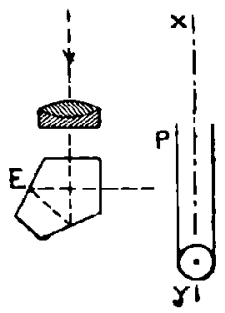

Fig. 106 .

La figure Io6 indique une transposition analogue, dans le cas d'un cliché sur pellicule $P$, à l'aide d'une équerre optique $\mathrm{E}$ (la figure doit être complétée par symétrie autour de $x y$ ).

2 5. Production de plusieurs clichès simultanés. - La photographie par le procédé trichrome exige la production de trois clichés géométriquement

1 Brevet fr. 326634 . 
identiques; la différence ne réside que dans la valeur relative des blancs et des noirs, et elle tient à l'interposition de glaces colorées en avant de l'image. On peut se proposer d'obtenir simultanćment les trois clichés. La figure 107 indique le dispositif préconisé par

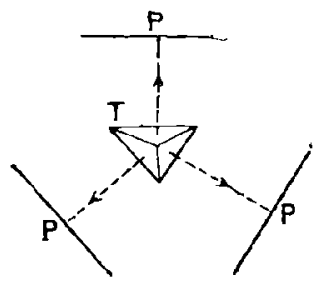

Fig. 107.

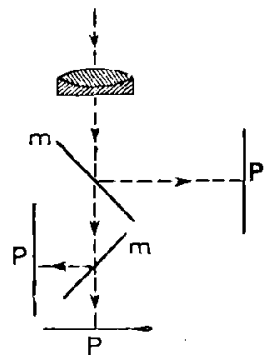

Fig. 108.

MM. Rigaud et Périère ${ }^{1}$; la figure 108 , celui de M. Nachet ${ }^{2}$.

Dans le premier, $T$ est un tétraèdre à trois faces réfléchissantes; l'objectif est sur une perpendiculaire au plan de la figure, face à $\mathrm{T}$. Dans le second, les $m$ sont des miroirs semi-transparents..

1 Brevet fr. 361883.

Brevet fr. 354781 . 


\section{CHAPITRE XII}

LYSTUMEYTS LT OHGANES DE MESUHE

DÉTERMINATION DES DIRECTIONS

\section{Lunettes-viseurs.}

2i6. Alidade. - Réticule. - On sait comment, dans l'antiquité, était optiquement matérialisée une direction, c'est-à-dire une ligne droite ; une petite ouverture circulaire, l'oxilleton derrière lequel se plaçait l'ceil ; à quelque distance en avant, deux fils croisés, dont l'intersection constituait un point matériel. Si l'organe visuel était très rapproché de l'œilleton, et si la croisée des fils cachait un objet ponctuel $\mathrm{A}$, celui-ci était sur l'une des directions passant par l'intersection des fils et l'un des points de l'ouverture circulaire. L'erreur angulaire géométriquement possible est égale à

$$
\frac{\omega+\varepsilon}{\delta} \text {, }
$$

$\omega$ éțant le diamétre de l'cilleton, $\varepsilon$ l'épaisseur d'un fil, \& leur distance à l'ouverture. L'erreur moyenne est Instruments optiques. 
beaucoup plus faible si l'observateur est assez habile pour bien centrer son oil afin de diminuer la valeur utilisable de $\omega$.

Cet instrument, l'alidade, était d'ailleurs un instrument relativement perfectionné, par rapport à ceux qui l'ont précédé et dont les organes de visée des fusils, de chasse ou de guerre, sont des exemples qui ont survécu.

L'alidade comportait, outre l'erreur géométrique, une erreur optique. L'intervalle $\delta$ est toujours assez faible. Pour s'assurer de la supęrposition en direction de la croisée des fils et del'objet, l'observateur est tenu à accommoder successivement et rapidement son oeil à la distance des fils et à celle de l'objet; il ne perçoit donc que la coïncidence des images réliniennes à des époques différentes et à l'aide d'un organe déformable, le cristallin. La précision de la visée n'est obtenue qu'en réduisant, au prix de la fatigue visuelle, les intervalles entre deux accommodations successives. L'erreur commise dépend donc de l'état physiologique de l'observateur.

Un savant italien, Divini, paraît être le premier (43) qui eût, en 1640 , l'idée de placer deux fils croisés au foyer de l'objectif de la lunette astronomique de Kepler. La cö̈ncidence, avec le point de croisement des fils, d'un objet, - étoile, par exemple, -indiquait que celui-ci était dans une certaine direction. Celle-ci est fixe dans l'instrument. Cette propriété suffit dans bien des circonstances.

$2 I_{7}$. Lunette à réticule. Axe optique.

L'intersection des deux fils du réticule, comme on dit aujourd'hui, est un point de la direction de la visée 
instrumentale. Un autre point est un point quelconque de l'axe de la lunette, si cet axe passe par la croisée des fils; et la coïncidence de l'image et du centre du réticule indique que colui-ci est sur l'axe de l'instrument.

Quand la croisée des fils est hors de l'axe, - et elle $y$ est toujours plus ou moins, - la direction de la vision est conjuguée, par rapport à l'oculaire, de la droite qui joint le centre $\mathrm{R}$ du réticule au centre $\mathrm{E}$ de l'ouverture d'émergence du système objectif (fig. 109).

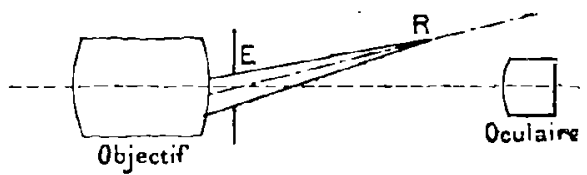

Fig. 109.

Elle passe done par le point oculaire et par l'image, généralement à l'infini, de la croisée des filsque donne l'oculaire. Sa détermination géométrique est aisée, mais ne présente aucun intérêt.

Pour un observateur qui laisse invariable tout le système optique, la direction visuelle est constante ; cela lui permet de mesurer des différences de direction, écarts angulaires.

On demande parf̊is davantage à l'instrument : on exige que, pour n'importe quel observateur, l'orientation de la lunette soit déterminée quand l'image de l'objet est au centre du réticule. Cette condition nécessite la coïncidence avec l'axe du système optique, de la droite ER, dite axe optique de l'instrument en tant qu'appareil de mesure.

Quand l'objectif est mince. E coincide avec le point 
nodal ou le centre optique; de là la définition habituelle de l'axe optique.

On arrive à ce résultat en assurant la coïncidence des deux axes en question avec l'axe géométrique de la lunette. Celui-ci n'est autre que l'axe idéal d'un cylindre parfaitement tourné, limité généralement à deux portées qui font partie constituante du corps de l'instrument. En faisant tourner la lunette autour de son axe géométrique, - il suffit de faire constarnment reposer les portées sur deux $\mathrm{V}$ fixes, - l'axe du système optique lui sera superposé si l'image d'un point fixe de l'espace reste fixe dans l'espace; c'est le constructeur qui assure cette propriété. La lunette étant ensuite munie du réticule, le point de croisement sera sur l'axc géométrique si, dans la même opération de rotation, ce centre du réticule est constamment perçu sur un même point fixe de l'espace.

2 18. Formes du réticule. - Le réticule est le plus souvent constitué par deux fils ténus et tendus à angle droit : fils de cocon, d'araignée ou fils métalliques, dont les extrémités sont fixées à la cire sur une plaque ouverte en son centre. Ces genres de réticules ne conviennent qu'à des instruments qui doivent être manipulés par des observateurs soigneux. Les : fils d'araignée donnent les réticules les plus fins.

Il est quelquefois avantageux de déterminer une direction non par la coïncidence du fil et de l'image, mais en amenant un point de l'objet à bissecter un faible intervalle; le fil réticulaire correspondant est alors dédoublé en deux fils parallèles, à l'intervalle demandé.

Quand la lunette-viseur doit être soumise à des mani- 
pulations qui détérioreraient un réticule à fils, on remplace celui-ci par une glace réticulaire portant deux traits opaques analogues à ceux des plaques micrométriques. Dans les lunettes à oculaire de Ramsden, on les a aussi tracés sur la face antérieure plane du verre collecteur; cette disposition, qui amène l'image sur le verre, nous paraît peu recommandable.

Avec les traits sur glace, le procédé de la bissection peut se passer de deux traits parallèles; il suffit d'interrompre la ligne réticulaire anx onvirons du centre; l'image do l'objet visé est alors amenée de façon à être bissectée par la ligne idéale qui prolonge les milieux des traits réticulaires.

Dans les instruments qui exigent la coïncidence des axes optique et géométrique, -- lunctles d'apparcils de nivellement, par exemple, - la plaque ou la glace réticulaire est mobile dans son plan; quatre via, deux à deux opposées, prenant appui dans Ja monture de la lunette, serrent les côtés de la plaque ou de la monture de la glace; une manoeuvre convenable de ces vis permet de donner au réticule la position demandée. On peut encore assurer la coïncidence des axes en déplaçant l'objectif, le réticule restant fixe.

Quand la lunette doit permettre le pointé à des distances différentes, le réticule est mobile dans le sens longitudinal; la coïncidence des axes doit alors être réalisée, s'il y a lieu, dans chaque position du réticule.

La mise au poinl, qui consiste à placer le réticule dans le plan de l'image objective, s'opère par déplacement de l'ensemble réticule oculaire, à l'aide d'un houton commandant soit un système pignon=crémaillère; soit, quelquefois, une bielle-manivelle qui agit alors sur 
l'objectif. Ce mouvement ne doit ètre ni trop lent ni trop rapide. Le colonel Goulier (39) fait observer que l'œil tolère entre le réticule el l'image un écart longitudinal $\varepsilon$ qui, dans le cas des lunettes astronomiques, peut être représenté par la formule empirique

$$
\varepsilon=0,0.1 \sqrt{f} \quad \text { [unité : millimètre] }
$$

$f$ étant la focale de l'objectif. Il indique que la correction de la mise au point exige $: I^{\circ}$ pour la mise au point de l'oculaire sur le réticule, qu'un déplacement de 1 millimètre de l'appui des doigts sur l'organe de prise (bouton moletté) corresponde à une variation longitudinale de $\varepsilon$ dans la position du système $; 2^{\circ}$ pour la unise au point sur l'objet, que le pas de la rainure hélicoïdale qui guide généralement l'oculaire soit égal à la focale de la partie située en arrière du réticule.

219. Éclairage du réticule. - Dans les observations de jour, la lumière diffuse éclaire suffisamment le réticule. Un éclairage artificiel est nécessaire pendant la nuit ou dans une chambre obscure. On peut obtenir une illumination suffisante en disposant devant l'objectif un écran annulaire qui renvoie la lumière diffuse d'une lampe quelconque : procédé indiqué par Biot (8); on peut faire aussi entrer la lumière par un petit miroir ou un petit prisme disposé dans le tube de l'instrument, par exemple, à hauteur des tourillons dans la lunette astronomique d'observatoire.

Mais il est préférable que l'éclairage se fasse d'arrière en avant, afin que l'œil de l'opérateur ne perçoive pas cette lumière, qui illuminerait anormalement le champ. On emploie souvent dans ce cas l'oculaire éclairant de Cornu (fig. I Io), portant une glace plane $g$ à $45^{\circ}$ sur 
l'axe, entre le réticule $r$ et le verre $v$ le plus rapproché de lui. Cette disposition exige un certain espace pour le logement de la glace et ne convient qu'à des oculaires assez faibles.

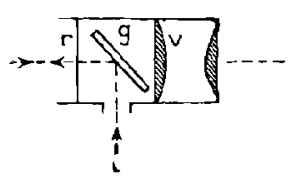

Fig. 110.

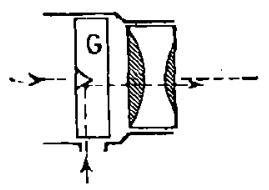

Fig. 111.

Le modèle de réticule suivant ${ }^{1}$ peut servir pour les observations de jour et de nuit; il est constitué par une glace $G$ assez épaisse (fig. I I I) portant des traits réticulaires fins, profonds et ouverts sous un angle convenable. A la lumière diffuse, ils paraissent opaques; pendant la nuit, une petite lampe, électrique par exemple, envoie un faisceau qui est parallélisé dans le plan et à l'intérieur de la glace; une portion de ce faisceau se réfléchit d'avant en arrière sur l'une des lévres du trait.

On a aussi indiqué ${ }^{2}$ la possibilité de constituer un point réticulaire lumineux par le sammet recourbé d'un cône de verre trìs effilé. La lumière serait conduite, - de la base au sommet, par ré-

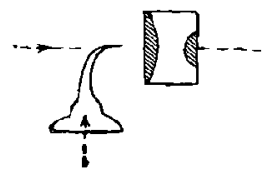

Fig. 112. flexion presque totale intérieure.

220. Emploi des viseurs. - Les viseurs, appa -

- Brevet fr. 345976.

2 Brevel fr. 356495. 
reils optiques munis de réticules, sont principalement des lunettes astronomiques, plus rarement terrestres, et des microscopes.

A l'exception des lunettes méridiennes, ces viseurs ne sont que des organes d'appareils mécaniques de mesure qui évaluent directement ou non l'angle formé par deux visées successives.

La lunette astronomique entre, à titre de viseur, d'abord dans les équatoriaux et les théodolites d'observatoire, puis dans la plupart des instruments géodésiques et topographiques qui mesurent des orientements ou des inclinaisons; enfin dans un grand nombre d'instruments de laboratoire, tant pour la vision ćloigncé que pour la vision rapprochée (goniomètres, spectromètres, cathétomètres, etc.).

Quant au microscope, il est surtout organe d'appareils de laboratoire; mais, à ce titre, il est plus souvent muni d'un micromètre.

Tant pour les Iunettes que pour les microscopes, l'oculaire utilisé dans les viseurs est celui de Ramsden, qui laisse l'image, objeclive en dehors de lui. La mise au point est plus aisće; et, en général, l'amplitude du champ peut être très réduite. Cependant, surtout dans les microscopes, on emploie quelquefois des oculaires Huygens; le verre d'cil est alor's mobile.

Le réticule peut évidemment s'adapter à la longuevue terrestre ou à la lunelte à prismes, lorsque le renversement de l’image offre des inconvénients. On a, en particulier, essayé bien des lunettes de ce genre, pour le pointage des bouches à feu de campagne. En dehors de la fragilité de ces appareils, la faiblesse de leur champ a conduit à leur rejet. Ils ont reparu, 
récemment, sous une autre forme. La lunette panoramique Goerz a été munie d'un réticule; celui-ci est fixe, comme dans les collimateurs; il est placé au foyer commun de l'objectif et de l'oculaire. La possibilité de choisir les pointeurs parmi les hommes à vue normale rend cette disposition acceptable. La société Krupp a fait breveter ${ }^{1}$ un dispositif analogue au précédent, qui contient un système optique mobile permettant de passer du pointage indirect au poinlage direct : le prisme en toit tourne de $90^{\circ}$ autour de son axe, et reçoit alors le faisceau direct envoyé par une équerre . optique. A la même idée, réalisće par des moyens différents, peuvent être rattachés la lunette de hausse de Zeiss ${ }^{2}$ et divers instruments de la Société Goerz.

\section{Collimateurs.}

22 I Principe du collimateur. - Une lunetleviseur, bien construite, de grossissement $G$, permet le pointé à un nombre de secondes près égal à 6o/G environ. Cette précision, qui n'est acquise qu'aux dépens du champ, n'est pas toujours nécessaire. Lorsqu'on veut augmenter ce dernier, mème en diminuant le grossissement, l'appareil devient plus volumineux qu'il n'est acceptable. .

Le problème est cependant susceptible d'une solution très simple quand G est égal à l'unitć.

Les astronomes et les physiciens emploient, depuis longtemps, pour matérialiser une direction, un appa-

1 Brevet fr. 359728.

- Brevet fr. 353449 .

IRIS - LILLIAD - Université Lille 1 
reil connu sous le nom de collimateur. Il comprend, montés invariablement sur un tube, une lentille convergente et, au foyer de celle-ci, un réticule. Le faisceau issu du centre du réticule donne un faisceau émergent parallèle, dont la direction est celle de la droite joignant la croisćc des fils au noud d'incidence.

Lne lunette astronomique, réglée pour observer un objet situé à l'infini en y rejetant l'image, se transforme en collimateur quand on en retire soit l'oculaire, soit l'objectif. La seule différence entre les deux procédés réside dans la longueur utile de l'instrument résultant.

Si l'on coupe un collimateur par un plan parallèle à son axe et voisin de celui-ci, et que l'on conserve seulement la partie de l'instrument portant la croisée réticulaire, l'oeil, situé en arrière du verre et placé de façon à être bissecté par le plan de section, verra à la fois directement le paysage et, à travers la lentille, le réticule. En réalisant la superposition de l'image du centre du réticule et d'un point du champ, la ligne de visće devient parallèle an faisceau donné par le collimateur, avec une approximation qui peut être déterminée.

A cet effet, supposons (fig. r r3), puisqu'aucun instrument n'est parfait, que le réticule soit à la très petite distance $\varepsilon \mathrm{du}$ foyer, - $\varepsilon$ positif quand la croisée des fils $\mathrm{R}$ est entre le foyer et la lentille; appelons a l'ouverture pupillaire et $h$ la distance de son centre à l'axe du système. 
L'image du rélicule se forme en $\mathbf{R}^{\prime}$ à la distance $p$ du point nodal d'incidence sensiblement égale à

$$
p=\frac{f^{z}}{\varepsilon} \text {. }
$$

Les directions possibles perçues sont (fig. I14) celles limitées par le cône du sommet $\mathbf{R}^{\prime}$ et s'appuyant sur les positions extrêmes de la pupille; soit MR' $\mathbf{U}^{\prime}$ ce cône.

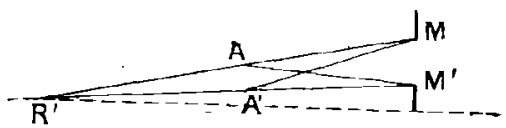

Fig. 114 .

L'image du réticule cö̈ncidera avec un point réel $\Lambda$ situé à la distance $q$, si A est compris dans ce cône. Les variations extrêmes possibles de la direction seront les droites $A M^{\prime}$ et $\Lambda^{\prime} \mathbf{M}^{\prime}$. Leur angle est sensiblement, en posant $\mathbf{M M}^{\prime}=b$ :

ou

$$
\begin{array}{cc}
\alpha=b\left(\frac{2}{q}-\frac{1}{p}\right), & \text { si } q<p, \\
\alpha \alpha=\frac{b}{p}, & \text { si } q>p .
\end{array}
$$

Quant à la valeur possible de $b$, elle est égale à $2 a$. pour un déplacement de l'œil dans le sens normal au plan de section du collimateur, et, pour un déplacement du centre de la pupille dans ce plan, elle est à peine supérieure à l'ouverture arrière $\omega$ du système, augmentée de $2 a$.

Les positions extrêmes ne laisseraient sentir qu'un faisceau infiniment délié. Pratiquement, on peut limiter à $0,25 a$ la première valeur de $b$, et à $\omega$ la seconde. 
22. Collimateurs à lentille simple. - Qu'il s'agisse d'un collimateur d'astronome, ou d'un collima teur-viseur, - c'est le précédent sectionné, comme il a été dit, - la précision avec laquelle il détermine une direction est, d'après les considérations exposćes ci-dessus, d'autant plus grande que la focale est plus considérable, et la mise du réticule au foyer plus parfaite.

Dans les instruments d'observatoire ou de laboratoire, c'est en allongeant les focales qu'on augmente la précision nécessaire. Mais, pour les collimateurs destinés au pointage des bouches à feu, les conditions d'emploi fixent la longueur maximum admissible pour l'appareil ; elle varie habituellement de 3o à 80 millimètres, atteignant rarement roo millimètres. Les focales sont du même ordre de grandeur. La précision de la mise au point et la conservation du réglage dans les manipulations constituent les difficultés à surmonter.

Le plus simple des collimateurs-viseurs, - il ne s'agira dorénavant que de ce genre d'instruments, est constitué (fig. II5) par

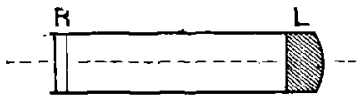

Fig. 115 . une lentille $\mathrm{L}$ convergente peu épaisse de faible ouverture (de 5 à ro millimètres environ) et une plaque ré ticulaire $\mathrm{R}$, montées aux extrémités d'un tube. Extérieurement, le deruier verre est dépoli; sur la face qui regarde la lentille, on a déposé une couche mince opaque, sur laquelle ont été tracés ensuite deux traits minces, rectangulaires, mettant le verre à nu. La croix constituée par ces deux 
lignes de foi est généralement disposée de façon que l'une des branches soit horizontale: celle-ci permet de pointer en hauteur, l'autre en direction. Pour la visée en direction, par exemple, l'observateur hoche la tête dans un plan vertical contenant la ligne de foi correspondante, de manière à apercevoir successivement l'objet visé et la branche de la croix : cette dernière ligne lumineuse est assez vive; la persistance de l'impression rétinienne suffit pour juger de la coïncidence demandée. Avec quelque habitude, les mouvements de tête sont imperceptibles.

La couche opaque de la glace-réticule est souvent constituée par du noir de fumée convenablement protégé ensuite, ou mieux par une mince couche d'argent, recouverte d'un vernis mat et sombre dont le rôle est d'éviter la réflexion de la lumière diffuse pénétrant par la lentille.

M. le commandant Estienne organise un collimateur fort simple (fig. I I 6 ) avec une demilentille plan-convexe $\mathrm{L}$, au foyer de laquelle se place la pointe $\mathrm{P}$ d'un petit cône métallique, en métal très réfléchissant (nickel), et à angrle un

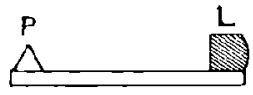

Fig. 116. peu ouvert pour que l'éclairage, soit suffisant en lumière zénithale. Pointe et lentille sont fixées invariablement sur une lamelle métallique.

Le stanhope, réglé de façon que sa face plane soit au foyer de la face courbe, - la longueur est alors $\frac{n r}{n} \frac{n}{-1},-$ et muni de lignes de foi; constitue un.collimateur simple et robuste, qui se présente habituellement sous la forme d'une courte baguette de verre à section carrée,

Instruments optiques. 
et qu'on emploie comme le premier instrument décrit dans ce paragraphe. On évite parfois la sujétion d'un travail précis du verre, cn exécutant séparément, et à des longrueurs nécessairement plus courtes, une baguelte lentille et une baguette porte-réticule. Le stanhope est ensuite róalisé par leur rónnion hout à bout avec interposition, quant au réglage, d'une cale mélallique ajourée d'épaisseur convenable; des jeux de cales variant de $\mathrm{o}^{\text {mim }}, 05$ en $\mathrm{o}^{\mathrm{mu}}, 05$, par exemple, permettent un réglage parfait.

223. Collimateurs achromatiques. - Les collimateurs à lentille simple ne sont pas achromatiques, et ceux à ligne de foi présentent de la distorsion. Cette dernière ne nuit pas au pointage tant que l'œil se tient dans le plan de la ligne réticulaire et de l'axe de la lentille; il est prévenu qu'il en sort par l'aspect que prend le trait lumineux. Dès lors, la visée perdant toute précision, la distorsion n'a d'autre inconvénient que de limiter pour l'ceil un déplacement qui n'est pas indispensable.

Le chromatisme n'a d'influence que sur la facilité $\mathrm{du}$ réglagge. Celui-ci revient à la détermination d'une focale, la précision de la mesure étant fixée. Ainsi, par exemple, si $f=50$ millimètres et $\omega=$ o 0 millimètres, la tolérance $\varepsilon$ sur la mise au point est, d'après les formules établies plis haut:

$$
\varepsilon=\frac{f^{2}}{3000 \omega}=\frac{50 \times 50}{30000}=0^{\mathrm{mrn}}, 08 .
$$

Or, dans une lentille simple, le foyer n'est déterminé qu'entre les deux points qui répondent aux limites des radiations perceptibles. Sans atteindre les extrèmes, on 
peut considérer en lumière hlanche les couleurs orangẻ et bleu. La différence cles foyers correspondants est assez sensiblement égale à

$$
\frac{n^{\prime}-n^{\prime \prime}}{n-}=
$$

$f$ étant la focale des rayons moyens d'indice $n$. Dans le crown ordinaire, où $n=1,5172$, et $n^{\prime}-n^{\prime \prime}=0,0$, la différence en question atteindrait $o^{\mathrm{mm}}, 33$, soit le quadruple de la tolérance admissible. Aussi convient-il de déterminer la focale pour une des radiations les plus agréables à l'oxil: lumière jaune ou vert franc. Pour la vérification de l'instrument construit, la lumière blanche suffit. La seule constatation qui s'impose est relative à l'absence de parallaxe dans le pointé; on s'en assure par visée soit directe, soil à travers une lunette de faible grossissement qui observe à la fois l'image réticulaire et l'ohjet.

On a cependant construit des collimateurs achromatiques, souvent constitués sur le modèle du stanhope, par un flint antérieur et un crown postérieur très épais.

22\%. Collimateur clair. - I.es collimateurs dont il vient d'être question présentent un inconvénient: pendant les opérations de pointé, une partie du champ est nécessairement masquée par la monture de l'instrument; la régrion ainsi éliminée est en outre la plus importante : c'est celle qui avoisine l'objet visé quand elle ne le comprend pas. On ne remédie à ce défaut quo par une éducation, simple à la vérité, mais qu'on peut se proposer de faire disparaître.

On ne saurait songer à disposer, en arrière du collimatcur, une lunette qui recevait à la fois l'imago des 
lignes de foi et celle de l'objet. Sans doute, verrait-ors ainsi la croix lumineuse se projeter sur le champ, la perception du collimateur ayant disparu; mais la solution, inacceptable avec une lunette terrestre ou à prismes, - il serait plus simple d'adapter un rélicule à celle-ci, — reste médiocre avec une lunette de Galilée dont l'ouverture efficace d'objectif est insuffisante quand le grossissement est faible, ot qui, pour un grossissement élevé, ne donnerail qu'un champ restreint et un réticule peu éclairé.

La solution idéale consisterait à projeter la croix Inmineuse dans un champ qui aurait une amplitude comparable à celui de la vision directe, toute perception du reste du collimatcur ayant disparu. Le colli-

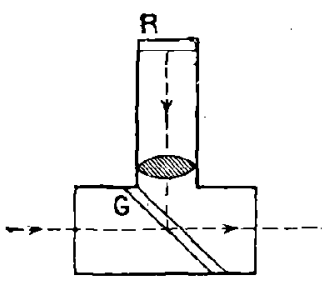

Fig. 117. mateur clair de Grubb (45, 46) (lig. I I ), basé sur uIt principe applicable à beaucoup d'instruments, remplit asser bien ces conditions : les faisceaux issus du réticule lumineux R. (croix transparente sur fond opaque) d'un collimateur astronomique dc courte focale (jo millimètres, par exemple) tombent sur une pile de glaces ou une glace $G$ faiblement arsentée. La seule imagre virluelle. fournie est celle de la croix, le reste du collimateur étant obscur; l'image est d'ailleurs à l'infini.

Le champ réel peut être très grand ; il est limité $I^{\text {In }}$ la pyramide, dont les arêtes, issues de l'œil, passent par le contour du iniroir. La clarté générale du champ, quoique affaiblic, reste suffisante. 
Le lieutenant Le Masne' a modifié cet instrument en supprimant l'argenture de la glace; celle-ci (fig. I I8) est disposée de façon que les faisceaux émergeant dı collimateur tombent sous une incidence de $7 \bar{j}^{\circ}$. La double réflexion produite sur les deux faces de la glace conserve à l'image de la croix lumineuse une intensité large-

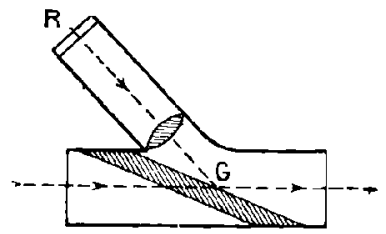

Fig. 118. ment suffisante; le degré d'inclinaison superpose con venablement les images produites par les réllexions multiples.

On peut constituer un collimateur clair relativement simple, en adjoignant à un collimateur ordinaire $\mathrm{C}$ un prisme de Govi G. comme l'indique la figure Ir9.

Le prisme de Govi (42) comprend denx prismes rectangles isoscèles collés par leurs faces hypoténuses de façon à constituer un cube; préalablentent, l'une des faces en conlact a été recouverte d'une couche d'or ou d'argent demi-transparente. Cette pel- . licule produit une réflexion suffisante,

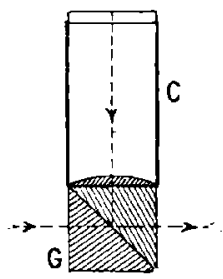

Fig. 119. tout en se laissant traverser par les rayons directs.

Le prisme de Govi peut ètre remplacé par le prisme qui cntre dans la lunette autocollimatrice du commandarnt Dévé (27o).

1 Brevet fr. 353356. 
225. Emploi des collimateurs. - Les collimateurs de laboratoire, - objectif astronomique et réticule à fils, - sont employés pour réaliser, dans de nombreux instruments, des faisceaux lumineux parallèles.

Dans les observatoires, deux courtes lunettes astronomiques à réticules peuvent ètre pointées l'une sur l'autre, objectif vers objeclif, comprenant un intervalle phus on moins grand; quand les points de croisée réticulaires coïncident, ils déterminent une ligne droite. Ln intercalant. dans l'intervalle des objectifs, une lunette d'instrument, on peut, par visées successives sur les deux réticules, étudier la flexion de l'organe en question.

Les collimateurs de visée sont, sous la forme stanhope, utilisés depuis longtemps dans les instru- ments topographiques; adaptés à un fil à plomb et réglés en conséquence, ils déterminent l'horizon de l'wil. Depuis une quinzaine d'arnées, ils ont reçu une autre application; ils remplacent la ligne de mire oilleton ou cran de mire et guidon - des bouches à feu, évitant par leur organisation la mise au point successive de l'œeil sur l'objet èloigné et sur le guidon, situé à un mètre au maximum de l'oxil; et cela, tout en conservarit, sous de légères restrictions, à la vision directe son champ naturel ; condition d'une importance pratique capitale, et dont la non-réalisation a fait rejeter tant de lunettes de pointage.

Entre un collimateur ordinaire et un collimateur clair, le choix ne serait pas douteux, s'il s'agissait d'un instrument destiné à des expériences. Les conditions d'emploi peuvent modifier la conclusion. I.e premier apparril est le plus rustique; cette constatation, 
dont les conséquences se devinent aisément, influe sur la décision à prendre. En outre, optiquement, le collimateur ordinaire est toujours prêt au service. La croix du collimateur clair ne se détache nettement que sur un fond rolativement sombre (terrain gris, prairies, bois, etc.) ; sur fond clair, sur le ciel, la perception ne s'obtient qu'à la suite d'un affaiblissement de la clarté du champ, produit pas l'interposition, en avant de l'œil, de verres absorbants (lames noircies). Ijne, pareille manouvre peat présenter plus d'inconvénients qu'une obstruction partielle du champ, à laquelle une éducation appropriće peut remédier, sans qu'il soit nécessaire ensuite de réfléchir au moment où l'action seule s'impose impérieusement. 


\section{CHAPITRE XII}

\section{IVSTRLMEYTS DE NESIRE DES AYGLES}

226 . - En dirigeant successivement une même Lunette à réticule sur deux points éloignés $\mathrm{A}$ et $\mathrm{B}$, le déplacement angulaire de l'axe optique est évidemment égal à l'angle sous tendu par AB du point d'intersection des deux posilions de cet axe oplique. Les appareils qui mesurent mécaniquement le déplacement angulaire en question sont d'usage courant et comportent de nombreuses variétés : cercles nuraux astronomiques, goniomètres de laboratoire, théodolites, tachéomètres topographiques, etc. Le cadre de cet ouvrage n'en comporte pas la description.

227 . Lunettes à micromètre. - On peut demander à l'organisation optique d'un appareil à mesurer des angrles d'assurer à la fois les opérations de visée el celle de lecture. Les lunetles à micromètre résolvent ce problème.

Si une lunette, - et plus généralement un système optique quelconque, - contient dans son champ deux points $A$ et $B$, la distance linéaire qui sépare leurs images $\Lambda^{\prime}$ et $B^{\prime}$ est une fonction bien détermince de 
langle $\mathrm{AQB}, \mathrm{Q}$ étant un point fixe, mais choisi arbitrairement, sur l'axe de l'appareil. Les paramètres de cette fonction dépendent des éléments qui définissent le système optique et la position du point $Q$, ainsi que de la distance séparant $Q$ des points $A$ et $B$. Le ròle du micromètre est de mesurer $A^{\prime} B^{\prime}$.

La propriété ci-dessus énoncée concerne l'une quelconque, réclle ou virtuelle, des images produites par le système optique. Sa mise en ceuvre est évidente quand on envisage l'une des images réelles. Il suffit alors de disposer dans le plan de collo-ci une graduation matérielle, étalonnée par avance, et, de la longueur interceptée sur elle par l'intervalle $\Lambda^{\prime} B^{\prime}$, déduire, en utilisant la fonction considérée plus baut, l'angle cherché. Un calcul préalable ou un étalonnage expérimental peut, d'ailleurs, réduire toute nesure à une simple lecture.

La graduation en question est le plus généralement tracée sur la face antérieure d'une glace à faces bien parallèles, dont ]'épaisseur est déterminée par des conditions de résistance. L'épaisseur des traits est d'autant plus faible, que leur écartement est moindre et que la précision exigée dans la mesure est plus élevée. Pour la détermination de ces grandeurs, dans les divers cas, on peut utiliser la remarque suivante: quand l'intervalle clair entre deux traits est vu, par l'ceil, sous un angle d'environ dix minules, un observateur moyen juge, sans autre point de repère, le $\frac{r}{5}$ de l'intervalle, et un observateur exercé arrive à l'apprćciation du $\frac{1}{10}$, correspondant à l'acuité visuelle.

Dans les instruments d'ordre courant, de précision 
moyenne, les traits micrométriques sont gravés à l'acide fluorhydrique et, ensuite, garnis de pâte grasse analogue à l'encre d'imprimerie. Si la précision exige des lignes minces et rapprochées, il faut les tracer au diamant plus ou moins chargé, suivant le cas; l'opacilé des traits ne résulte alors que de la destruction du poli à l'endroit qu'ils occupent.

Ces micromètres tracés au diamant peuvent être très fins, mais leur visibilité est faible; ils exigent un éclairage convenablement orienté.

Pour des instruments de laboratoire, nous avons utilisé avantageusement des micromètres photographiques sur pellicule de collodion canoutchouté.

Un dessin du micromètre, élabli sur papier avec une amplification de 25 à 30 , est photographié à sa grandeur définitive sur plaque au collodion lumide, par deux réductions successives; la surface est ensuite recouverte d'une couche de solution de caoutchouc, la pellicule détachée et emprisonnćc entre deux plaques, ajouróes en leur centre, qui constituent la monture du micromètre.

On peul obtenir ainsi des traits purs, suftisamment noirs, et dont l'épaisseur n'atteint pas $0^{\text {mam }}, 0 \mathbf{r}$. La lecture de ces micromitres n'occasionne pas la fatigue qu'on ressent dans l'emploi des nuicromètres à traits au diamant. Leur déformation est insensible si on prend des précautions pour leur montarre, et si l'on s'en sert exclusivement dans un laboratoire. la pellicule est d'ailleurs plus résistante qu'on ne pourrait le croire à priori; elle supporte le balayage des poussières à l'aide d'un pinceau. Enfin, elle offre l'avantage de ne pas produire, gràce à sa très faible épaisseur, une deuxième image du nuicromètre, due à la réflexion, quand l'éclairage est très intense. 
228. Particularités $d u$ micromètre. - La face graduée de la glace micrométrique devant être dans le plan de l'image, les moindres poussières qui sont déposées sur elle arrêtent totalement les faisceaux qui atteignent ces corpuscules opaques nécessairement situés en leurs sommets. On peut éviter cet inconvénient, assez grave dans lẹs appareils destinés à des observations à l'extérieur, en reportant les poussières en avant, ce que l'on obtient en doublant la plaque micrométrique par une autre glace collée sur la graduation.

Certaines lunettes, qui sont avant tout des instruments d'observation, doivent cependant, - les conditions d'emploi peuvent l'imposer, - être constamment munies d'un micromètre. Si celui-ci n'est pas toujours entretenu dans le plus. grand étal de propreté, la lunette pourra n'òtre qu'un médiocre appareil d'observation, si elle doit être à tont instant prête pour co rôle. 'Tel est le cas des lunettes de batterie. Il est possible d'éviter en partie cet inconvénient, lorsque la graduation ne doit être étendue que sur la moitié du champ, en constituant la plaque micrométrique seulement par une glace demi-circuläire qui n'occupe que la moitié du champ. Il faut alors que le plan diamétral qui la limite soit bien nef et rigoureusement perpendiculaire aux faces de la plaque; sinon le champ est coupé par une ligne bleuâtre, plus ou moins longue et franche, provenant de la déviation des faisceaux centraux, le bord de la glace agissant comme un prisme non achromatique.

Quand l'obstruction du champ, par le micromètre, doit être évitée, et qu'en outre les mesures n'exigent 
pas une grande précision, on pent avoir recours au micromètre périphérique du commandant Daubresse ${ }^{1}$. Il consiste en une mince pellicule de celluloïd, ouverte en son centre et qui remplace ou double le diaphragme de champ; le bord interne de l'ouverture porte les graduations nécessaires, qui sont d'ailleurs obtenues photographiquement, les pellicules sensibilisées étant d'usage courant.

229. Origine et évolution $d u$ micromètre. - La première idée du micromètre paraît ètre due à Huygens, qui, en 1659 , pour mesurer la distance angulaire de deux étoiles ou d'un astre, introduisait dans le plan de l'image objective de la lunetle astronomique un triangle métallique allongé. De la Jargeur du triangle qui couvrait l'astre ou l'intervalle des étoiles et de la focale de l'objectif, il fléduisait l'écart angulaire cherché. En 1662 , Malvasia remplaça la lame triangulaire d'ITuygens par un grillage de fils d'argent très déliés, formant des carrés égaux. I’idée avait été utilisée pour le même objet, par Moestlin, avec la chambre obscure. C'est, au fond, le type du micromètre sur glace. En I 667, Auzout ne laissa subsister du grillage de Malvasia qu'un fil fixe et un autre qu'il rendit mobile sous l'action d'une vis micrométrique : c'est le micromètre des lunettes d'observatoire (64).

Aucun micromètre ne peut être adjoint aux systèmes optiques ne formant pas d'image réelle. Pourtant. Galilée a mesuré, à l'aide de sa lunette et avec une précision remarquable, des intervalles angulaires (43). Son procédé a même continué à être employé après

1 Brevet fr. 317356.

IRIS - LILLIAD - Université Lille 1 
l'invention de la lunctte astronomique. Il était basé sur la connaissance du champ apparent de la lunette et du grossissement. La valeur du charnp réel en était conclue; elle était faible, en raison du grossissement considérable, 33, de l'instrument. L'amplitude du champ réel servait d'unité pour l'appréciation des écarts angulaires.

23o. Micromètre à fil mobile. - Le micromètre d'Auzout est encore en usage dans les lunettes de précision; il est généralement doublé d'un réticule destiné au pointé.

Il comprend un cadre portant un fil de réticule (fil d'araignée, par excmple); le fil est successivement amené sur les deux points qui déterminent l'angle ; son mouvement est commandé par une vis à pas très fin portant un tambour gradué, qui sert à évaluer les fractions du pas. En observant la gaduation du tamhour à chaque visée et comptant le nombre de tours de vis entre les deux pointés, on en déduit l'écart linéaire des fils : le pas de la vis a été taré d'avance. Généralement, dans le plan du diaphragme se trouve un peigne dont les dents sont à un écartement sensible. ment égal au pas de la vis; son róle est, non de permettre une lecture précise, mais d'éviter une crreur dans le décompte du nombre entier des tours de vis.

Les meilleurs micromètres à fils mobiles accusent, à la lecture, des déplacenients de $\mathbf{o}^{\mathrm{m} / \mathrm{n}}$,oor. Il est rare que l'on puisse compter sur une pareille précision; l'imperfection du filetage s'y oppose généralement.

Avec ce genre de micromètre, l'instrumęnl qui en est muni.se rapproche des appareils goniométriques considérés au $\$ 226$. 


\section{3 r. Emploi de la lunette à micromètre.}

Les instruments destinés aux mesures angulaires et que l'on munit de micromètres sont généralement des luneties astronomiques, terrestres ou à prismes.

Le micronètre à fils est surtout réservé aux lunettes astronomiques; il donne des écarts linéaires que l'on convertit en angles en utilisant les constantes de l'instrument, ou en ayant recours à un tarage préalable.

Le micromètre sur glace convient à toutes les lunettes. Ia graduation est habitucllement chifflée de telle sorte que l'angrle soit lu directement, en fraction de circonférence ou par sa tangente.

Dans une lunetic astronomique ou dans une lunette à prismes, la seule place du micromètre est dans le plan focal de l'objectif, si l'observation doit toujours avoir lieu sur des objets éloignés; sinon, il est dans le voisinage de ce.plan, et comporte la possibilité d'un léger déplacement pour sa mise au point sur l'image objective. Dans une Iunette terrestre, il peut être indifféremment placé à proximité de l'une ou l'autre des images réelles ; en le disposant près de l'oculaire. les intervalles des traits répondant à un écart angulaire donné sont maximums. Si les objets à examiner sont toujours éloignés, il est préférable de le placer au foyer de l'objectif; de cette façon. tout l'oculaire terrestre peut être constitué rigidement et. par suite, dans les meilleures conditions pour la correction des aberrations. La mise au point s'effectue simultanément sur l'image et sur le micromètre par déplacement d'ensemble de tout l'oculaire.

La graduation d'un micromètre sur glace pourrait se faire. surtout quand la lunetle est destinée à la vision 
lointaine. que le champ est restreint, par voie de calcul. Un intervalle micrométrique $\varepsilon$ répond à un écart angulaire de $\frac{\varepsilon}{f}, f$ étant la focale du système qui produit l'image réelle sur le micromètre. Yais il est toujours plus simple et plus exact de procéder par étalonnage direct, lequel est d'ailleurs nécessaire quand l'instrument n'est pas orthoscopique.

Pour les mesures très précises, un tableau de corrections doit ètre établi, pour que l'on puisse tenir compte des déplacements du micromètre, si celui - ci n'est pas fixe.

232. Collimateur-goniometre. - Le collimateur de visće peut être adapté à la mesure des angles; il suffit de remplacer les lignes de foi par des traits parallèles. A l'écartement $s$, l'angle correspondant à un intervalle est le quotient de $\varepsilon$ prar la focale du collimateur.

Sous la forme très courte du stanhope, et avec des traits noirs sur fond clair, fixé à un perpendicule, le collimateur sert aux topographes à mesurer de faibles pentes. Le champ en est peu étendu : 5 à $6^{\circ}$; l'erreur possible sur l'angle est au moins de $\boldsymbol{2}^{\prime}$.

Pour la mesure de grands écarts angulaires, le commandant Estienne ${ }^{1}$ a fait construire, sous le nom d'octant de poche, un instrument basé sur le principe du collimateur, dont l'amplitude atteint $45^{\circ}$ (autrement dit 800 millièmes) ${ }^{2}$. Il comprend (fig. I 20 ), en principe,

1 Brevet fr. 321814 .

2 Le millieme de l'A 'tillerie est une unité angulaire qui équivaut a la $1600^{e}$ partic d'un angle droit; soit 6.25 centigrades. Sa tangente trigonométrique est sunsiblement égale à 0,001 . 
une lentille épaisse plan-convexe, de faible épaisseur dans le sens perpendiculäire à l'axe optique, mais de grande ouvertıre; une graduation convenable est tracée sur la surface focale

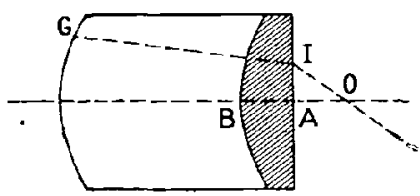

Fig. 120. qui passe par le foyer situé du còté de la face courbe, et qui est ici concentrique à cette face. L'oil se place du côté de la face plane du verre.

Pour explorer le champ considérable de l'appareil, l'seil doit tourner autour de son centre de rotation $O$; et le pinceau qui, issu d'un point G de la graduation, émerge en pinceau parallèle, doit passer par ce contre O. En posant $\mathrm{O} \lambda=\delta, \Delta B=e, \mathrm{IOA}=\theta$, désignant par $r$ le rayon de la face convexe de lit lentille, par $n$ l'indice du verre, on a aisément, entre ces éléments et la focale $\mathrm{BF}=f$ de la lentille, la relation :

$$
f=\frac{r}{n-1}=\frac{1}{n-1}\left[e+i \sqrt{\frac{n^{2}-\sin ^{2} \theta}{\cos ^{2} \theta}}\right] .
$$

$f$ étant conslant, ¿ ne pourrait l'ètre, puisque $\theta$ entre dans cette expression. Mais il suffit que la relation soit vérifiée pour une valeur de $\theta$ plus rapprochée des extrémités de la graduation que du centre; car, dans le voisinage du foyer, la position de l'œil sur l'axe est indifférente.

Il est avantageux, pour l'emploi de l'instrument, d'en rendre la longueur minimum, et par suite de réduire $f$. Or on ne peut guère agir que sur $n$, et la discussion de la relation précédente montre qu'on doit prendre 
des verres très réfringents : leur conservation limite $n$ à la valeur $x, 6$ r. La focale $f$ ne pent donc théoriquement descendre au-dessous d'une certaine valeur. En faisant $e=8$ millimètres (valeur pratique), $\delta=\mathrm{r} 3,6+5=\mathrm{r} 8^{\mathrm{ran}}, 6$ (distances du centre de rotation à la pupille, et de celle-ci au verre), $\theta=20^{\circ}$, on trouve $f=64$ millimètres.

Toutcfois, dans la pratique, on obtient des résultats satisfaisants en employant du crown ou de la glace, réduisant $f$ à 50 millimètres environ, et remplaçant la surface focale par un cercle centré sur B et passant par le foyer. Ce sont les donnécs du commandant Estienne, qui, en outre, termine (fig. I2I) sa lentille par un prisme à réflexion totale PL, faisant corps avec elle, de façon que l'observateur place l'octant contre son cil O perpendiculairement à la direction du plan de visée;

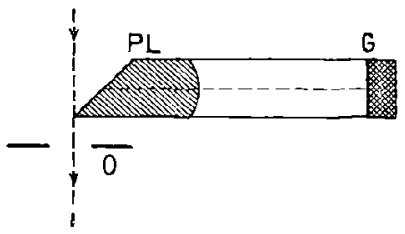

Fig. 121. la pupille est bissectée par l'arête du prisme et aperçoit, dans le champ el à la hauteur des objets visés, l'image de la graduation. Celle-ci est formée de traits noirs de a à 3 millimètres de hauteur sur métal blanc. L'octant permot la mesure des angles à $r 5^{\prime}$ près environ. Suspendu à la façon d'un perpendicule ou muni d'un niveau dont l'image est perçue en mème temps que la graduation, il constitue un appareil très pratique pour la mesure des angles de site et des pentes.

233. Sextant. - L'angle de deux points lunineux, vus d'un point $O$, est déterminé par les pinceaux qui 
en émanent el qui passent par 0 . Les instruments précédents évaluent l'angle en mesurant l'intervalle de ces pinceaux à ane distance connue du sommet 0 . On peut aussi considérer l'un d'entre eux comme une déviation de l'autre et se proposer d'annuler cotte déviation par un systène optique qui, assumant leur coincidence, superposera les images des objets, et dont la déformation ou le déplacement sera fonction de cette déviation, c'est-à dire de l'angle à mesurer.

L'anuulation de la déviation peut être obtenue par des miroirs plans, par des prismes ou par des lentilles.

A la première catégorie appartiennent les apparcils du genre sextant.

Un miroir plan $m$ (fig. 122) couvre, à quelques centimètres de distance, la moitic droite d'un objectif de lunette astronomique L. Par la moitié gauche de la

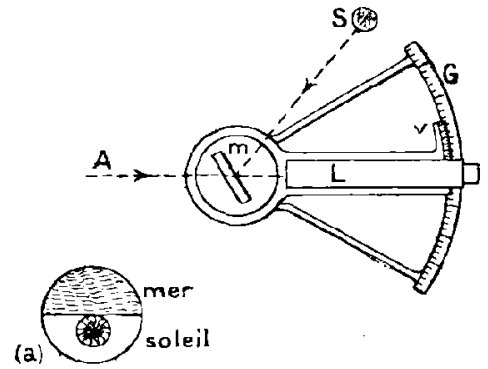

Fig. 122. lunette, l'observateur voit directement le point $\Lambda$; par la moilié droite. il luj superpose l'image réfléchic dis point $\mathrm{S}$, situé en arrière, tel que l'angle $\Lambda \mathrm{mS}$ soit le double de celui formé par l'axede la lunette et la normale au miroir. C'est le dispositif imaginé par IJooke en 1664 , réinventé par Grandjean ${ }^{1}$ en $\Upsilon_{7} 32$, et, sous

1 Grandean. Instrument pour observer les hauteurs en mer. Machines et inventions approuvées par l'Académie royale des Sciences de Paris, t. VI, 1735. 
le nom de secteur catadioptrique, par Segner (81). Le miroir est monté sur un plateau qui porte une graduation concentrique $G$, dont l'intervalle entre deux divisions vaut un demi-degré sur la circonférence, et par suite équivaut à un degré d'angle d̀ évaluer. La lunette porte un vernier $v$, est mobile antour de la droite du miroir perpendiculaire au plateau et au plan de la graduation. Le mouvement relatif de la lunette et du reste de l'appareil permet de ríaliser la coïncidence des images de deux objets quelconques, dans certaines limites de champ. La figure (a) montre comment on peut déterminer ainsi la hauteur apparente du soleil.

234. Sextant à deux miroirs. - L'instrument précédent exige que l'on tourne le dos à l'un des objets à observer; la recherche de son image peut être pénible. A jeu près simultanément $(\mathbf{8 1})$ et d'une façon indépendante, New ton et Hadlcy, en $173 l$, évilèrent cet inconvénient en employant deux miroirs (fig. I 23). L'un M est solidaire de la graduation, et ne couvre, quant ì sa face argentée, que la moitié de l'objectif; par la partie transparente, l'ob-

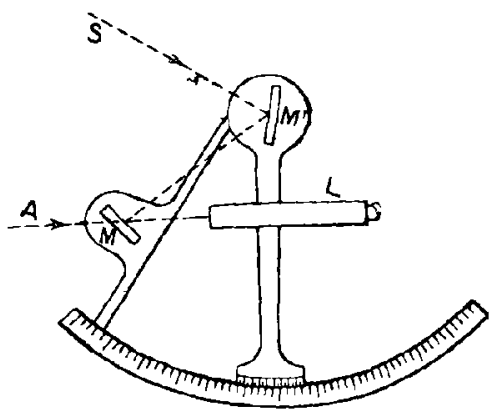

Fig. 123. jet A est vu directement; par sa partie spéculaire, il transmet d̀ la 
lunette l'inage de l'objet $S$, une première fois réfléchie par un miroir $1 \mathbf{l}^{\prime}$ solidaire de la lunette. L'angle visuel des deux objots est égal au double de l'angle des plans des niroirs : la démonstration de cette propriété est élémentaire. L'appareil d'Hadley, désigné sous le nom d'octant, permettait la mesure des angles de o à $45^{\circ}$. Newton, en portant l'angle à $60^{\circ}$, lui donna le nom de sextanl, qui indique maintenant l'instrument à deux miroirs, quelle que soit l'amplitude de la graduation.

Jes avantages du sextant sont de permettre, avec un instrument ramassé, et par suite portatif, la mesure de grands angles, sans que l'immobilité de l'appareil soit nécessaire. La lunette à micromètre exige la fixité ou la lecture simultanée, par suite délicate, en deux points de la graduation; pour le sextant, il suffit que la double image ne sorte pas du chanıp de l'instrument. Ia précision qu'on peut obtenir avec le sextant est limitée à la deni-minute, au plus à 20". La présence d'images parasites dues aux réflexions sur les faces non argentées des miroirs, le défaut de planéité de ceux-ci, qui sont de faibles dimensions, ne permettent pas une précision plus grande. Pour l'obtenir, il est d'ailleurs nécessaire que l'instrument soit réglé de façon que les plans des miroirs soient perpendiculaires à celui de la graduation, et que l'are optique de la lunette soit parallèle à ce miême plan.

Les sextants de la marine, qui servent à la détermination des hauteurs angulaires des astres, - l'un des objets est alors la ligne d'horizon naturelle ou l'image du second réfléchic sur un horizon artificiel, - sont munis de lunettes astronomiques. I es instruments destinés 
aux observations terrestres comportent des lunettes redressant les images.

Le champ du sextant ne peut guère dépasser $60^{\circ}$, qui répond à un angle de $120^{\circ}$ pour les miroirs; en augrnentant ce dernier angle, les pertes par rétlexion sur la surface du verre s'acentucnt ainsi que la production d'images parasites. On peul toutefois mesurer des angles plus considérables en rejetant le faisceau émané du point vu indirectement, à $90^{\circ}$, par exemple, de sa direction avant qu'il atteigne le premier miroir; il suffit d'interposer sur son trajet un prisme déviateur. C'est la solution adoptée par le commandant Aubry dans son scxtant-télémètre ${ }^{1}$.

Les miroirs plans du sextant peuvent ètre remplacés par des prismes à réflexion totale. Ia figure r s!́t donne un exemple ${ }^{2}$ de cette disposition, employce dans un certain nombre d'instruments. Le prisme $P$, invariablement fixé à la lunette, couvre la moitić de son objectif; le prisme $\mathrm{P}^{\prime}$,

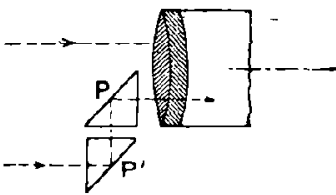

Fis. $12 \%$. situé au-dessous de $\mathrm{P}$, peut tourner autour d'un axe parallèle à la direction commune aux arêtes des prismes, qui sont d'ailleurs normales à l'axe de la lunette ainsi qu'une face réfringente de P. la mouvement de rotation est convenablement amplifié pour faciliter la lecture. Ia précision de la mesure peut être assez grande, et atteindre celle du pointé; les angles mesurés sont faibles, 4 a $5^{\circ}$; leur grandeur est limitée

1 Brevet fr. 289079.

2 Gana. Brev. fr. 336879 et addition 3965 . 
par la valeur de la réflexion totale; au-dessus des nombres indiqués, l'image doublement rếfléchie diminue rapidement d'intensité.

235. Sextant goniomètre à prismes. - M. le commandant Daubresse ${ }^{1}$ a imaginé, sous le nom de goniomètre portatif à prismes, un sextant dans lequel les miroirs sont remplacés par des prismes rectangles et isoscèles agissant à la façon des prismes de Wollaston

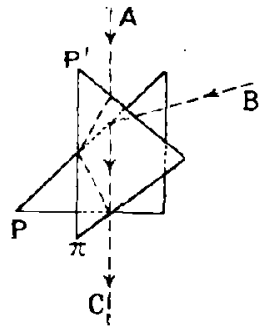

Wig. 125.

(fig. I 25). Le prisme inférieur P est fixe; l'autre $\mathrm{P}^{\prime}$ mobile, et son déplacenent mesure lc double de l'angle visuel, comme dans le sextant. La figure indique la marche des rayons issus de $A$ et de $B$, qui se superposent suivant le pinceau unique $\mathrm{C}$. Celui-ci est reçu par une lunette placée en face de l'arête convenable $\sigma$ du prisme fixe; le plan de contact des dẹu prismes contient

l'axe optique de la lunette. Celle-ci est coudée de façon à opérer un redressement partiel des images; en la remplaçant par un viseur uniquement formé d'un prisme de Wollaston agissant à $90^{\prime \prime}$ de ceux de l'appareil, le redressement optique est complet, mais le grossissement s'abaisse à l'unité. L'avantagr de cet ingénieux dispositif est de permettre la mesure des angles jusqu'à $\mathbf{1} 80^{\circ}$, tout en évitant les images parasites des miroirs. Cette propriété compense largement l'inconvénient dù aux pertes de clarté par réflexions sur les faces d'entrée et de sortie, et celui tenant à la nécessité de

1 Brevet fr. 317597, de'1902. et Revue d'Artillerie (février 1902). 
centrer convenablement l'wil pour assurer le même éclairement aux deux images. L'instrument n'a d'ailleurs qu'un faible volume.

236. Goniomètres à réfraction. - La superposition des faisceaux envisagée plus haut (233) peut être produite par un prisme intercalé sur l'un d'eux, de façon à le ramener en coïncidence avec le faisceau reçu directement, c'est-á-dire reçu en dehors de toute action du prisme.

La déviation produite par celui-ci doit être égale à l'angle $\alpha$ que forment les pinceaux dáns la région de leur trajet où le prisme déviateur est interposé; car les faisceaux directs peuvent avoir déjà été rendus plus ou moins convergents par un système optique approprié, lunette par exemple.

Les formules classiques permettent de calculer $x$ sous les formes:

oll

$$
\begin{aligned}
& x=f(i, a, n), \\
& x=g(c, a, n),
\end{aligned}
$$

$a$ est l'angle du prisme; $i$ et $e$, ceux d'incidence et d'émergence; $n$, l'indice du verrs.

En général, la mesure de $i$ ou de $e$ serait aussi pénible que la mesure directe de $x$. Aussi doit-on rendre constante l'une de ces quantités; la préférence doit être accordée à $e$. La mesure de $x$ revient alors à celle de l'angle $a$ du prisme. Elle exige, par suite, l'emploi de prismes à angles variables, de diasporamètres.

Une déviation un peu forte conduit à des prismes très ouverts; la netteté des images exigerait l'achromatisation individuelle des deux éléments dy diasporamètre; mais la distorsion des images subsisterait. Aussi 
n'a-t-on guère utilisé de prismes à grands angles.

Quand l'angle du prisme est assez faible pour que l'on puisse remplacer les sinus par les angles, _ et cela dépend du degré de précision requis dans la mesure, - les relations précédentes se réduisent à :

$$
x=(n-i) a \text {. }
$$

La constance de $i$ ou de e n'est plus nécessaire, pourvu que ces angles restent faibles. C'est surtout dans ce cas, mesure de petits angles, que le diasporamètre a êté employé.

Le procédé primitif consiste à interposer sur le trajet de l'un des faisceaux des prismes successifs dont les angles vont régulièrement en croissant, suivant une progression arithmétique dont la raison est égrale ou peu supérieure à la précision demandée. Si la coïncidence des imagres ne peut être obtenue par l'emploi d'un prisne déterminé, leur situation relative se modifie pour deux prismes successifs d'angles $x^{\prime}$ et $\alpha^{\prime \prime}$. La moyenue de ces angles, corrigée au besoin par l'appréciation des rapports entre les distances angulaires des images, dans les deux cas, donne la valeur cherchée $\alpha$ Inn appareil basé sur co principe a été employé : il comprend une série de très petits prismes montés sur la circonférence d'un plateau, et dont les angles varient de minute en minute.

Mais c'est surtout le diasporamètre de Rochon qui est utilisé, celui de Boscovich ne se prêtant pas à la réalisation de petits angles. Deux prismes identiques, d'angle faible $a$, sont accolés; si on leur dome un déplacement relatif, d'amplitude angulaire $\theta$ autour de la normale à leur face commune, ils constituent un 
prisme résultant dont l'angle au sommel $a^{\prime}$, qui est celui des faces extérieures de l'ensemble, est déterminé par la relation rigoureuse :

$$
\sin \frac{a^{\prime}}{2}=\sin a \sin \frac{\theta}{2},
$$

qui devient, pour des angles au sommet faibles :

$$
a^{\prime}=2 a \sin \frac{\theta}{2} \text {. }
$$

L'angle 0 est nul quand les faces extérieures sont parallèles : cette condition détermine l'origine de la rotation.

Le diasporamètre a surtout été utilisé comme organe télémétrique.

237. Micromètre parallèle. - A la méthode précédente peut se rattacher l'emploi de glaces épaisses à faces parallèles, prismes d'angle nul. La relation gémérale $\alpha=f(i, a, n)$ se simplifie à cause de la nullité de $a$, et la mesure de $i$ est facilitée par ce fait que cet angle est grand pour de faibles valeurs de $\alpha$.

$\mathbf{S i}$, devant une lunette à réticule pointée sur $\mathbf{A}$, on interpose une grace à faces parallèles d'épaisseur e et d'indice $n$, dont la normale fait avec l'axe de l'instrument un angle $\omega$, le point $B$, dont limage se forme sur le réticule, est à un intervalle linéaire, $\varepsilon$, de $A$ déterrniné par les relations :

$$
\varepsilon=e \frac{\sin (\omega-r)}{\cos r}, \quad \sin r=\frac{I}{n} \sin \omega .
$$

Dè cette longueur $\varepsilon$, on conclura l'écart angulaire des points $\Lambda$ et $B$, situés à une distance finie, généraInstruments optiques. 
lemont faible, d'un point déterminé de l'axe de la lunette.

Porro, qui a revendiqué la paternité du disposilif, plaçait la grlace entre l'objectif et l'oculaire (73).

238. Prismes biréfringents. - Ia superposition des pinceaux d'écart angulaire assez faible peul être obtenue en interposant sur leurs trajets des prismes composés, taillés dans des substances biréfringentes uniaxes (quarlz, spath d'lslande, cristal de roche), à faces extérieures parallèles. I.es deux variétés principales de ces organes sont le prisme de Rochon et celui de Wollaston.

Le prisme ou micrometre de Rochon est formé (fig. I 26 ) de deux prismes trian-

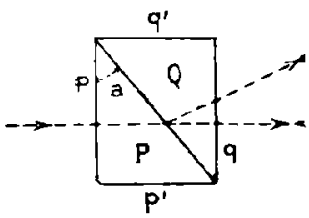

Fig. 126. gulaires 1' et Q, habituellement en quart, de même angle $a$, collés ensemble par leurs faces hypoténuses, à l'aide de térébenthine. I'ensemble constitue un parallélépipède. La face active $p$ du prisme $P$ est perpendiculaire à l'axe optique du cristal; les arêtes de $Q$ sont parallèles au mème axe. Un faisceau parallèle, - onde plane, - tombant normalement sur la face active $p$ de $P$ reste nnique et conserve la même direction dans la traversée de ce prisme; à la surface de séparation des deux prismes, le rayon ordinaire poursuit sa marche directe; l'autre y est dévié, ainsi qu'à la face de sortie de Q. L'angle z des faisceaux émergents est donné par la relation :

$$
\sin z=\sin a\left[\sqrt{n^{\prime}}-n^{2} \sin ^{2} a-n \cos a\right] \text {. }
$$


$n$ et $n^{\prime}$ étant les indices ordinaire et extraordinaire de la substance.

L'angle \& croît très lentement avec $a$. A vec le spath, le rayon extraordinaire est éliminé par la réflexion totale quand l'angle $a$ atteint $63^{\circ}$ environ.

Dans le prisme de Wollaston, le prisme $\mathrm{P}$ est taillé de façon que l'axe cristallographique soit parallèle au plan de la face active et normal aux arètes; le prisme $Q$ est le même que précédemment. L'ensemble n'est autre qu'un prisme de Rochon dont les faces inactives $p^{\prime}$ et $q^{\prime}$ sont devenues actives. Lo pinceau tombant normalement sur $p^{\prime}$ donne deux faisceaux superposés, ordinaire et extraordinaire, de même direction que le rayon incident. A la surface de séparation, les ondes se séparent; mais le second prisme, agissant comme polariscope, élimine deux des faisceaux. ne laissant que l'extraordinaire issu de l'ordinaire, et l'ordinaire provenant de l'extraordinaire. Ces faisceanx sont de nouveau déviés à l'émergence sur la face de sortie; leur écart angulaire, $\delta^{\prime}$, ou déviation due à l'appareil, est, $a$ étant l'angle d'un prisme (égal au complément de l'angle $a$ du prisme de Rochon), donné par les relations :

$$
\begin{aligned}
& \delta^{\prime}=\dot{s}_{1}+\delta_{2} \\
& \sin \grave{\partial}_{1}=\sin a\left[n^{\prime} \cos a-\sqrt{n^{2}-n^{\prime 2} \sin ^{2} a}\right] \\
& \sin \partial_{2}=\sin a\left[\sqrt{n^{12}-n^{2} \sin ^{2} a}-n \cos a\right] \text {. }
\end{aligned}
$$

Le prisme de $\mathrm{W}$ ollaston donne une déviation plus grande que le prisme de Rochon; mais les images ne sont pas achromatiques. Dans le dispositif de Rochon, l'image non déviée, seule, n'est pas irisée. 
En supposant ò et $\delta^{\prime}$ faibles, on a, pour les déviations données par les deux prismes, la relation :

$$
\delta^{\prime}=\grave{\delta}+\sin a\left[n^{\prime} \cos a-\sqrt{n^{2}-n^{\prime 2} \sin ^{2} a}\right]=\grave{o}+\grave{s}_{1} .
$$

239. Duplication de l'image. - Le prisme de Rochon a ćté utilisé pour réunir, du còté de la face $p$, deux faisccaux tombant sous un angle convenable sur la face opposée du parallélépipède ${ }^{1}$.

llais Rochon s'en est surtout servi pour faire coïncider, quand deux faisceaux tombent à peu près normalement sur la face $p$, l'image ordinaire de l'un avec l'image extraordinaire de l'autre. Le prisme est placé (fig. 127) entre l'ob-

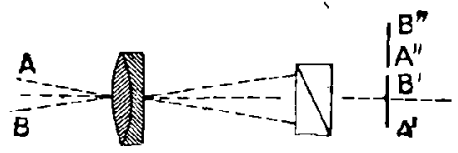

Fig. 127. jectif d'une lunette astronomique et l'imagre réelle qu'il fournit de deux points 1 et $B$, dont l'angle apparent est $r_{1}$. Il se forme alors dans le plan focal deux images ordinaires $\Lambda^{\prime}$ et $B^{\prime}$ et deux images extraordinaires $\Lambda^{\prime \prime}$ et $B^{\prime \prime}$. En déplaçant le prisme le long de l'axe, on peut produire

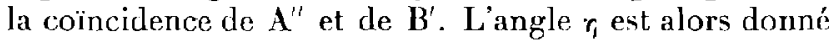
en fonction de $x$, distance de la face de sortie du prisme au foyer, de l'angle de duplication $\delta$ évalué plus haut, et de la focale $f$ de l'objectif par la relation :

$$
\lg n=x+{ }_{f}^{\operatorname{tg} \delta}=l i x
$$

1 La maison Zeiss (brevet fr. 376157) associe deux prismes de Rochon portant en outre chacun une lame biréfringente formant polarisateur circulaire. L'ensemble constitue un prisme a ancle variable. 
$k$ étant une constante. Une graduation all hoc fait connaître le déplacement $x$ effectué.

Arago, pour la mesure des diamètres angulaires des astres, plaçait le prisme en dehors et près de l'oculaire. L'angle $r_{i}$ est alors donné par l'équation

$$
\cdot \operatorname{tg} \eta=h^{\prime} \stackrel{\operatorname{tg} \delta}{-\bar{a}},
$$

$G$ étant le grossissement de la lunette et $k^{\prime}$ une constante qui dépend de la position du prisme et qui est détermince expérimentalcment: Pour satisfaire à cette relation, Arago faisait varier 3 en interposant des prismes dont l'angle de duplication croissait de $3 \mathrm{O}^{\prime \prime}$ en $3 \mathrm{o}^{\prime \prime}$, et modifiait en vulre $\mathrm{G}$ en utilisant un oculaire à focale variable. Biot attribue à ce dispositif une supériorité sur l'héliomètre (6).

La précision des mesures est limitée par le chromatisme de l'image extraordinaire, quoique l'angle de duplication soit toujours faible.

2'fo. Héliomètre. - La duplication des images peut ètre obtenue par l'emploi de lentilles (fig. 128). Si deux objectifs $0, o^{\prime}$ astronomirgues identiques ont leurs axes parallèles, les images des points éloignés A et. $B$ seront confondues

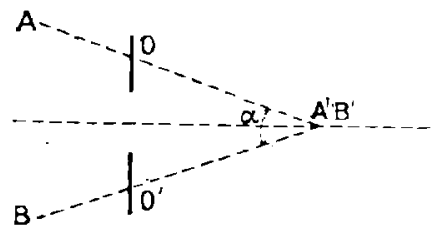

Fis. 128. quand on aura :

$$
e=-2 f \operatorname{tg} \frac{x}{2}
$$


La mesure de $\boldsymbol{x}$ résulte de celle de $e$. C'est le sys tème inaginé par Bouguer, en 1748 (8); il ne pernet d'ailleurs que la mesure d'angles supérieurs à la limite donnée par l'équation ci-dessus, où l'on remplace $e$ par' le diamètre de l'objectif (monture comprise).

En modifiant ce disposilif, Dollond en a fait un instrument tout différent, l'héliomètre, qui permet la mesure d'angles aussi petits qu'on le désire.

Le micrometre de Dollond, comme on l'appelle aussi, est constitué par une lunette astronomique ou terrestre dont l'objectif est scié suivant un plan diamétral que l'on fait coüncider avec celui qui passe par les points $A$ et $B$ visés. La formule indiquée ci-dessus s'applique a cet instrument; $e$ désigne la distance, qui peut atteindre zéro, des centres des cleux moitiés de l'objectif. Une vis micrométrique à tambour gradué, commandant le déplacement des demi-objectifs, mesure leur écartement; le mouvement déplace, en général, chaque verre symétriquement par rapport à la posilion moyenne de coïncidence des centres.

2ł1. Emploi des goniomètres optiques. L'emploi des lunettes à micromètre est général et commode pour la mesure des petits angles; la grandeur de ceux-ci no peut évidemment dépasser celle du champ réel de l'instrument, et ce demier prendrait des dimensions trop exagérées si, conservant la précision, on voulait agrandir le champ embrassé. L'usage de ces instruments nécessite leur immobilité.

Les appareils à duplication ou à coïncidence n'exigent pas cette condition. Les sextants sont utilisés lorsque la précision des mesures doit être faible, les angrles étant alors assez considérables. L'emploi des prismes 
monoréfringents convient à la nesure de petits angles; celui des prismes biréfringrents et de l'héliomètre donne, dans ce cas, des résultats généralement plus précis, car la taille régulière des petits prismes ordinaires est assez. délicate, et la disposition de ces organes n'est arbitraire qu'entre certaines limites, qui se rapprochent d'autant plus que l'on demande plus de précision dans la mesure.

Instruments de laboratoice, de topographie, d'astronomie, ces dispositifs se retrouvent avec de légères variantes dans les apparcils de mesure des distances. 


\title{
CHAPITRE XIV
}

\author{
IVSTRLYFYTS DE VESLRE MDIHECTE DES LONGLELHS \\ TÉLEVITRRS
}

242. Dans la très grande majorité des circonstances, toute mesure d'une longuenr, d'une distance, est liée a la résolution numérique on matérielle d'un triangle dont un côté an moins est connu, qu'il soit donné ou mesuré. Les instruments optiques n'interviennent, en réalité, dans colte opération que pour l'évaluation des angles nécessaires à la détermination du triangle. Leur rôle est surtout utile quand il s'agit soit de faibles intervalles, de l'ordre du millinètre par exemple, soit de distances qui ne descendent guère au-dessous de 20 à 30 mòtres, et qui somvent sont supérieures à quelques containes de mètres. Enlre ces limites, les procédés mécaniques sont plus mpides, aussi précis et moins onćreux.

Tous les instruments de mesures angulaires envisn gés au chapitre précédent sont applicables à l'évaluation des longueurs et des distances. Leur choix dépend des conditions de la mesure et de la précision exigée. inversement, tout macrometre peut servir de gonio- 
metre; ces doux mots sont pris dans leur acception étymologique. La différence d'organisation ne tient qu'à leur mode normal d'emploi; mais elle suffit souvent pour transformer en un instrument nouveau et original un appareil deja connu.

243. Le problème télémétrique 1 - Pour la mesure des distances à l'aide d'appareils optiques, télémètres, un côté seulement du triang]e est généralement mesuré; nous l'appelons la base. L'une des extrémités de la longueur à évaluer est le troisième sommet du triangle; l'autre estrímité est connue de situation relativement d̀ ce triangle. Celui-ci est donc seul à considérer pour la précision des opérations.

Dans le triangle AMN (fig. r29), de base $\mathbf{M N}$, la géométrie donne les relations:

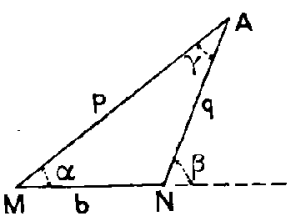

Fig. 129.

$$
\begin{aligned}
& p=b \frac{\sin \beta}{\sin (\beta-\alpha)}, \\
& q=b \frac{\sin \alpha-}{\sin (\beta-\alpha)} .
\end{aligned}
$$

L'erreur zp possible sur $p$, par exemple, est donnée par l'équation :

$$
\frac{\partial p}{p}=\frac{\partial b}{b}+[-\cot \beta+2 \cot (\beta-z)] \delta=,
$$

en admettant la mème erreur possible $d \equiv$ sur chaque angle.

1 Voir à ce sujet: $(22)$. 
On en déduit aisément que, pour une base $b$ donnée, l'orientation la plus convenable à lui donner serail déterminée par la celation:

$$
\sin 2(\beta-\alpha)-2 \sin 2 \beta=0 .
$$

Cette équation ne pourrait être utilisée que si on connaissait d'avance une valeur approchée de $p$. Il suffira ici de remarquer que, si le triangle est isoscèle, le premier membre de cette équation se réduit à

$$
2 \sin \gamma+\sin 2 y,
$$

$\because$ étant la parallaxe de la base vue du point A. La quantité ci-dessus est très faible dès que $\mathbf{A}$ est assez. éloigné. De là l'avantage des triangles isoscèles.

Quand la base peut être quelconque' sans que la précision relative de sa mesure diminue, il y a naturellement avantage à la prendre la plus grande possible, dans le but d'accroitre les angles $\alpha$ et $\beta$, tout en res tant dans, le voisinage de la forme isoscèle, afin d'augmenter $\beta-\alpha$.

Dans le cas du triangle isoscèle, les formules précéderites deviennent :

$$
p=\frac{b}{2 \cos \alpha}=\frac{b}{2 \sin \frac{\gamma}{2}},
$$

et $\quad \frac{\delta p}{p}=\frac{\delta b}{b}+\operatorname{tg} \alpha \delta \alpha=\frac{\delta b}{b}+\cot \frac{y}{2} \delta \gamma \cdot\left(b^{\prime}\right)$

Si le triangle est reclangle en $\mathrm{N}$, Ies mômes formules se transforment dans les suivantes:

$$
p=\frac{b}{\cos \alpha} \quad q=b \operatorname{tg} \alpha=b \cot \gamma
$$




$$
\begin{gathered}
\frac{\partial p}{p}=\frac{\partial b}{b}+2 \operatorname{tg} x \delta x, \\
\frac{\partial q}{q}=\frac{\partial b}{b}+(x \operatorname{tg} x+\cot x) \delta x .
\end{gathered}
$$

Le coefficient 2 est nécessaire, parce que l'angle droit n'est déterminé qu'à l'erreur angulaire près $\delta \alpha=\delta$.

244. Emploi de triangles quelconques. L'évaluation de la distance d'un point $\mathbf{M}$ à un point $\mathbf{A}$, qu'on tient pour inaccessible, peut s'effectuer par les mesures successives d'une base $\mathrm{MV}=b$, et des angles $\alpha$ et $\beta$. C'est le cas des opérations géodésiques de plus ou moins d'envergure. I.es organes optiques qui interviendront ne sont ici que des lunettes à réticule. Les formules ( $a$ ) doivent êlre appliquées rigoureusement pour la résolution du triangle.

L'emploi de théodolites ou instruments analogues visés dans ce cas assure une grande précision aux mesures, mais n'est pas compatible avec la rapidité des opérations. On peut se contenter, dans certaines circonstances, d'une approximation assez large : par exemple, dans les levers topographiques expédiés ou dans la détermination des distances du tir des bouches à feu. Si la base peut être convenablement orientée, suffisamment étendue et assez exactement mesurée, les angles peuvent n'ètre évalués qu'avec une approximation voisine de la minute. L'usage du sextant est alors indiqué.

Angles et base peuvent être mesurés par des opérateurs différents ; alors, comme dans les mesures géodésiques, c'est le supplément de $\beta$, c'est-à-dire l'angle à la base du triangle, qui est directement évalué, chaque 
station déterminant avec le point $\mathrm{A}$ un angle dont l'autre station est le sommet. Mais, dans un grand nombre de cas, le même opérateur exécute toutes les mesures. Le point A est toujours sur l'un des côtés de chaque angrle; l'autre côté passe par un point fixe choisi sur le prolongement de la base et dans le sens que celle-ci est parcourue entre les deux mesures. Le sextant doit permettre la mesure de grands angles; il pourra être un goniomètre a prismes, si on ne veul le munir d'une équerre optique. Son organisation sera presque purement mécanique, dans le but de faciliter visées et calculs. Ainsi le sextant-téléncitre Aubry comporte une lunette de Galilée, de faible grossissement, qui redresse les images, et une règle à calcul permettant de résoudre rapidement les équations $(a)$; de plus, la lecture des angles s'effectue par tambour et non par vernier, plus sujet à erreur. Dans le goniomètre du commandant Daubresse (235), on a signalé l'emploi d'une lunette ou d'un viseur; la résolution du triangle est effectuce par un abaque.

Les appareils de ce genre sont nombreux ; leur organisation mécanique, plutôt que leur composition optique, les différencie.

\section{Emploi de triangles rectangles. - Le} calcul du triangle est singulièrement facilité quand, la base étant petite par rapport à la distance, les angles à la base sont voisins de $90^{\circ}$. I.es formules (a) donment alors, comme daus le cas du triangle isoscèle ou du triangle rectangle :

$$
p=\frac{b}{\gamma} \text {. }
$$

$\mathrm{Si}$, en outre, la longueur de la base $b$ est déterminée, 
une simple lecture donne, en lieu et place de $\gamma$, la distance cherchée $p$.

Parmi les instruments qui conviennent à ce genre d'opération, nous citerons d'abord (59) le télométre ${ }^{1}$ Croulier (fig. I 30 ), destiné à la mesure des distances de tir, et encore réglementaire dans l'artillerie de campagne. L'appareil est, en principe, à deux opérateurs opérant simultanément; il résout un triangle sensil)lement rectangle, et comprend deux instruments diffé-

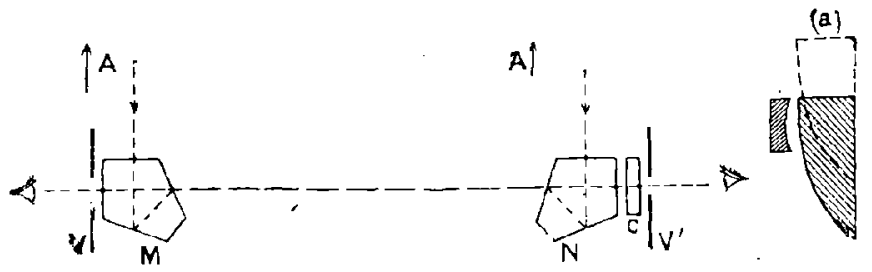

Fig. 130.

rents. L'un M n'est autre qu'une équerre optique (26I), prisme réfléchissant comportant sous l'angle de $45^{\circ}$ deux faces argentées; son rôle est de placer le sccond appareil $\mathbf{N}$ à $90^{\circ}$ de la ligne $\mathbf{A M}$; cette disposition est réalisée quand la ligne de foi tracée sur le voyant $V^{\prime}$ de l'appareil $\mathbf{N}$ est dans la direction de l'image du but $A$ vu par double réflexion dans le prisme. La ligne de visée directe dans $M$ est assurće par une lunette ou un viseur à fente remplissant le rôle de réticule. L’instrument $\mathbf{N}$ porte les mêmes organes que $\mathrm{M}$; en outre, devant le prisme se trouve un système correcteur $c$ qui permet de modifier la direction du

1 Ténos, but.

Instruments optiques. 
faisceau venant de $\mathbf{A}$, et qui comprend (fig. ı 3o, a) deux lentilles plan-convexe et plan-concave. à même rayon de courbure, les faces courbes en regard. Quand les axes des deux lentilles coïncident, lour ensemble agit comme une glace à faces parallèles. Si le viseur de $\mathbf{N}$ est dirigé sur la ligne de foi du voyant $\mathbf{V}$ de $\mathbf{M}$, le point $A$ ne peut se superposer à celle-ci que s"il est à l'infini; dans le cas contraire, un déplacement relatif d'une lentille par rapport à l'autre assurera la coïncidence, et ce déplacement $\equiv$ donnera la parallaxe $\gamma$ par l'équation $f \epsilon=\gamma, f$ étant la focale de la lentille convergonte. Ce système équivaut à un prisme déviateur à angle variable.

L'instrument indique la distance avec la précision que lui assignent les formules, s'il est manić par des observateurs exercés qui peuvent assurer, à des instants déterminés, la simultanéité des visées sur le même point du but et sur les lignes de foi respectives:

Un même opérateur, muni de l'instrument $\mathbf{N}$, peut exécuter toutes les opérations : déterminer d'abord en M la direction de la base, la lentille déviatrice étant placće au repère infini (axe des lentilles en coüncidence); puis, se transportant en $\mathbf{N}$, en en mesurant la distance, opérer, avec le système correcteur, la coïncidence du but et d'un siơnal placé préalablement en $\mathbf{M}$.

246. - Un grand nombre d'instruments sont basés sur le mème principe : déviation à $90^{\circ}$ avec dispositif correcteur. En particulier, le télémètre Gautier, dans lequel l'équerre optique est formée de deux miroirs à $45^{\circ}$, le systène correcteur étant un diasporamètre de Rochon; le télémètre Amici, qui produit la déviation à $90^{\circ}$ par un prismé newtonien à réflcxion totale et. 
assure la correction par une lentille divergente, coupée comme dans l'héliomètre. Dans d'autres appareils, l'image directe est reçue sur un réticule de lunette, la rotation de miroirs ou de prismes nécessaire pour assurer la coïncidence de la seconde inage est mesurée mécaniquement. L'exécution de la plupart de ces instruments est malheureusement aussi simple que leur conception; elle ne donne pas la précision qu'on pourrait en attendre.

Les formules $(c)$ s'appliquent à ces lélémètres. En admettant que l'on puisse atteindre pour le pointé la précision de la minute $\left(\Sigma \alpha=\frac{1}{3000}\right)$, on obtient, pour l'erreur absolue possible sur la distance, la relation

$$
\delta p=\frac{p}{b} \delta b+\frac{-2}{3000} \frac{p^{2}}{b} .
$$

Cette formule n'est suffisamment approchée que si $p>$ io $b$.

247. Emploi du triangle isoscèle. - L'emploi d'un triangle isoscèle a conduit à une solution remarquable au point de vue optique; elle est donnée par le Distanzmesser de Bauernfeind (59). Co petit instrument est du genre des équerres optiques; c'est un simple prisme, en verre (fig. I31), à peu près rectangle et dont la facc hypoténuse PQ estargentée; l'angle en $P$ est égal à $45^{\circ}+\varepsilon$; l'angle en $O$, double de $P$, vaut $90^{\circ}+2 \Xi$. Un faisceau issu de $\Lambda$,

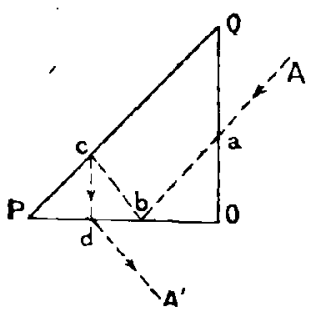

Fig. 131. qui suit un trajet tel que $\mathrm{A} a b c d \mathrm{~A}^{\prime}$, sort en faisant un 
angle de $90^{\circ}-2 \varepsilon$ avec sa direction primitive, et cela, quelle que soit son incidence sur la face $Q 0$, pourvu que le faisceau reste dans la section droite du prisme. La démonstration de cette propriété s'effectue en appliquant la loi de Descartes et celle de la réflexion sur miroirs plans.

Le prisme permettra donc de déterminer une direction $\mathrm{MN}$ laisant avec la distance $\mathrm{ML}$ à mesurer un angrle de $90^{\circ}-2 \varepsilon$. On se transportera ensuite sur MX jusqu'au point $\mathbf{N}$ qui donnera une direction $\mathbf{N A}$ faisant avec NM l'angle $9 \mathrm{o}-2 \varepsilon$; il suffira de viser,

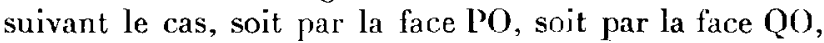
les réfractions et réflexions se produisant toujours sur les mêmes faces et dans le mème ordre relatif.

Dans ces conditions, la distance $\mathbf{M} \mathbf{H}=p$ est, pour un prisme donné, proportionnelle à la base $\mathbf{M N}=b$; le coefficient de proportionnalité n'est autre que l'inverse de $2 \sin 2 \varepsilon$.

D'autres prismes présentent les mèmes propriétés que le précédent; entre autres, le prisme pentagonal de Bauernfeind (38), qui comporte quatre parcours différents, et le prisme telémètre Souchier ${ }^{1}$.

248. Appréciation des procédés télémétriques utilisant une grande base. - lies appareils précédents nécessitent des mesures à deux stations plus ou moins éloignées. Avec un observateur unique, opérant successivement, leur emploi est incompatible avec la rapidité de la mesure, et se trouve en défaut quand le but est mobile; avec deux observateurs, il est indispensable que les visées soient simultanées sur un

1 Revue d'Artillerie, t. XLI, 1893.

IRIS - LILLIAD - Université Lille 1 
but nobilc, et même sur un objet fixe si les instruments sont tenus à la main; il faut, de plus, que les deux opérateurs s'entendent sur le point exact à viser : une erreur d'une dizaine de centimetres est souvent inadmissible. Or cette difficulté de préciser le but, quand celui-ci n'est pas un véritable signal, est parfois assez grande. Même à un observateur unique qui se transporte de l'une des extrémités de la base à l'autre, il arrive fréquemment de perdre ou d'échanger le but, tant l'aspect du paysage peut se modifier, même pour un déplacement du point de vue d'une quaranlaine de mètres.

- Parmi les appareils qui n'exigent qu'une station, les uns supposent connue la grandeur d'une base située, qui passe par le but; ce sont les lunettes à micromètre, dites alors stadimétriques, et les instruments qui mettent en jeu la duplication des images, et qu'on peut appeler télémètres à décalage d'images; les autres, auxquels on réserve habituellement la qualification de télimètres monostatiques, portent en eux-mêmes la base linéaire nécessaire à toute évaluation métrique.

\section{Lunettes stadimétriques.}

2/9 - Une lunette à micromètre mesure des angles. Si son axe est normal au milieu de la baso quí passe par le but A, l'angle sous-tendu par la base connue $b$ déterminera la distance de celle-ci à un point fixe de linstrument. 
En effet, on a dans ce cas (fig. I32), par les formules classiques des lentilles simples, la relation

$$
\mathrm{D}-f=f \frac{b}{{ }^{n}},
$$

donnant la distance du milieu de $b$ à l'objectif $O$. Le triangle résolu est

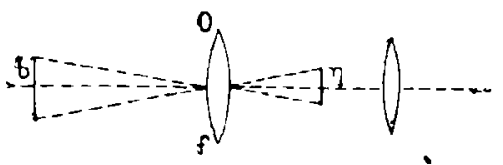

kig. 132. ici isoscèle; et les conditions pratiques permettent de confondre les distances à A et à un autre point de la base.

En général, le micromètre est divisé en parties égales, et c'est le nombre $h$ de divisions couvertes par l'image do la base que lit l'observateur. Si chaque intervalle entre denx traits vaut $\frac{\mathrm{l}}{m}$ de millimètre, la formule ci-dessus peut s'écrire :

$$
\mathrm{D}-f=m f \frac{b}{h}=k \frac{b}{h},
$$

$l k$ étant une constante de l'instrument.

Lorsque l'éloignement de l'objet est assez grand, on peut négliger $f$ devant $\mathrm{D}$, c'est-à-dire faire usage de la formule

$$
p=k \frac{b}{h} \text {. }
$$

C'est elle qui est appliquée dans l'évaluation des distances de tir; en campagne, $b$ est la hauteur d'un homme, d'un cavalier; dans le tir à la mer, la base est la hauteur de mâture (distance entre la ligne de 
flottaison et un point remarquable d'un màt ou l'extrémité d'une cheminée). Ta précision qui ressort, pour la distance, de la mesure tient surtout au degré d'exactitude dappréciation de la base; Jes bases sensiblement verticales sont préférables; on a rarement une idée suffisante de l'orientation des bases horizontales.

${ }_{25} 5$. Lunette anallatique. - La formule $(a)$ du paragraphe précédent doit être conservée, lorsque la focale $f$ de l'objectif ne peut être négligée devant la distance qui sépare A de l'appareil. Le cas se présente dans les levers topographiques à grande échelle, où les distances des cheminements sont faibles et doivent être obtenucs avec une grande précision (celle qui répond, sur le dessin topographique, à $\mathrm{o}^{\mathrm{mm}}, \mathbf{1}$ ).

La base $b$ est ici constituée par une mire ou règle divisée, dite stadia, tenue verticalement ou horizontalement : position et longueur sont bien connues.

La dislance qui intéresse esl celle qui sépare la míre de l'axe vertical de rotation de la lunette, lequel est placé à l'aplomb du sommet de cheminement. Un calcul serait nécessaire pour passer de la valeur de D à la distance en question.

Porro, en introduisant entre l'objectif et le micromètre une lentille convergente, dite verre anallatiseur, convenablement calculée, a modifié l'instrument de façon à reporter sur l'axe de rotation vertical l'origine des distances $p$, telles que la formule de leur calcul se présente sous la forme simple

$$
p=l_{i}^{\prime} \frac{b}{h},
$$

$l^{\prime}$ étant une constante.

La théorie sommaire de cette limette anallatique 
résulte de la figure i 33 . Les formules des lentilles simples donnent:

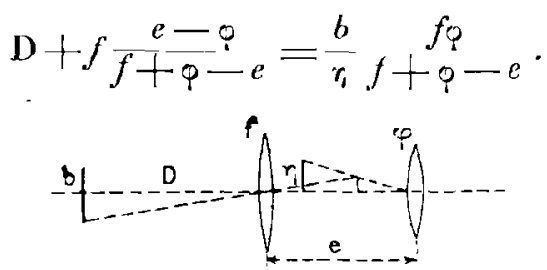

Fig. 133 .

Cette relation se réduira à la précédente si l'on fait

$$
p=\mathrm{D}+f \frac{e-\varphi}{f+\varphi-e}, \quad k=\frac{f \varphi}{f+\varphi-e} .
$$

La distance $p$ est donc comptée à partir d'un point de l'axe optique situé en arrière de l'objectif à l'inter. valle $\lambda$ donné par

$$
\therefore \quad \lambda=f \frac{e-q}{f+q-e} .
$$

Cette équation est la relation d'anallatisme. Flle déterminera, $\lambda$ étant la distance de l'objectif à l'axe vertical de rotation $X$ de la lunette, la place ou la focale du verre anallatiseur $V$. On peut remarquer que $\mathrm{X}$ est lo foyer conjugué par rapport à l'objectif du foyer principal antérieur du centre optique de la lentille V.

Ces lunettes ont en réalitó un oculaire négatif à trois verres; les formes des deux lentilles extrêmes sont différentes de celles de l'oculaire Iluygens, dans le but de corriger les aberrations sphériques (41). 


\section{Télémètres à décalag̣e d'imag̣es.}

25 I. - Si, à l'aide d'un appareil de duplication, à axe sensiblement horizontal, - pour fixer les idées, on observe un objet ayant une hauteur verticale convenable, on peut, pour une certaine position de l'organe mobile ou déformable (prisme, demi-lentilles, miroirs), disposer les deux images produites dans le prolongement l'une de l'autre, et telles que le sommet de l'une coïncide avec la base de l'autre. L'instrument mesure alors la grandeur apparente, angulaire, de l'objet. Si la hauteur de celui-ci est connue, une formule, en principe identique à celle des lunettes à micro mètre, permettra d'en déduire la distance de l'appareil.

Pratiquement, la distance à mesurer est toujours assez grande; il n'y a pas liea d'envisager les dimensions de l'instrument. Le triangle à résoudre est sensiblement isoscèle. La relation

$$
p=b
$$

suffit à la théorie des télémètres en question.

Ces appareils se substituent aux lunettes stadimétriques quand la fixité nécessaire à ces derniers ne peut être assurée.

Tous les instruments de duplication usités dans les laboratoires de physique ou les observatoires astronomiques ont étć transformés en télémètres à décalage d'images. Les modifications apportées sont surtout d'ordre mécanique: disposilion des organes en vue de réduire ]'encombrement et de faciliter la mise en main, 
lectures métriques, amplification des déplacements, adjonction d'abaques qui suppriment tout calcul, etc.

252. Prisme monoréfringent. - La duplication produite, concurremment avec la vision directe, par un prisme monoréfringent d'angle faible, a été appli quée jadis dans la marine. L'apparcil élait constitué par une série de prismes, à angles régulièrement écheIonnés, disposés sur la circonférence d'un plateau. L'oil se plaçait de façon à fairc bissecter sa pupille par le plan passant par le milieu du but et l'arête du prisme.

Ce principe a été repris, récemment, par le commandant Gérard ${ }^{1}$, sous la forme du diasporamètre de Rochon.

Les deux prismes $\mathrm{P}$ com-

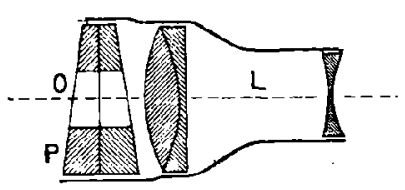

Fig. 134. posants (fig. I 3/i), d'angle faible, comportent, dans leur partie centrale, une ouverturecirculaire $\mathrm{O}$ centrée sur l'axe de rotation du système. Ene lunette de Galilée L permet l'observation des images par l'ouverture et $\grave{a}$ travers les verres. L'opérateur effectue la duplication en tournant les deux prismes en sens inverses et du mème angle. L'observation ayant lien surtout sur des objets verticaux (hommes, cavaliers, mâtures, etc.), l'égalité des rotations est suffisamment décelée par le maintien de la verticalité apparente des images : l'œil est moins tolérant que l'instrument n'est précis.

La transformation du résultat angulaire $\gamma$ en dis-

1 Brevet fr. 352028. - La Nature, 23 mars 1907. - Revue d'Infanterie, 1907. 
tances $p$ est effectuée automatiquement par les montures des prismes qui, avec un index mohile et dont la position se règle d'après la valeur de $b$, constituent une règle à calcul.

253. Prisme biréfringent. - Le prisme biréfringent ou micromètre de Rochon a été utilisé comme moyen de duplication, en particulier, dans la jumelle du colonel Souchier. Le prisme est ici placé en arrière de l'oculaire d'un des corps d'une jumelle; il peut se rabattre dans la bonnctte, quand la jumelle doit être . utilisée comme instrument d'observation.

Le prisme est dans une position invariable, longitudinalement, par rapport à l'oculaire. Le décalage des images ne peut donc, sauf exception, être complet; les images empiètent l'une sur l'autre. L'observateur juge du rapport $p$ entre la parlie détachée de l'une des images et la partie recouverte par l'autre image. La longueur $b$ de la hase constituée par l'objet est réduite dans le rapport $p$ en question.

254. Lentilles fendues. - La marine a adopté depuis longtemps (59), comme télémètre à décalage, l'héliometre de Dollond, sous la forme donnće par Lugeol, officier de vaisseau, et avec la modification apportée, dans la mesure du déplacement des lentilles, par l'opticien Lorilleux; ce déplacement est amplifié par un dispositif multiplicateur dont l'organisation est uniquement mécanique.

Le principe de la duplication par demi-lentilles n'implique pas que celles-ci constituent l'objectif; la séparation des images se produira si le système de décalage est interposé en un point quelconque du trajet du faisceau. En 1823 , Amici a proposé de 
placer les demi-lentilles entre l'objectif et l'image réelle, dans une lunette astronomique on terrestre. L'avantage tient à l'emploi de lentilles de petites dimensions ; l'inconvénient est de réduire le déplacement de l'organe mobile.

Giraud-Teulon (35), en 1875 , a opéré la duplica. tion en sectionnant loculaire. Le déplacement est alors égal à l'étendue de l'imagre objective; comme dans l'héliomètre de Dollond, les défauts d'aberration sont moins perceptibles.

255. Miroirs et prismes à réflexion. - Le sextant peut être employé, comme appareil de décalage, dans les mèmes conditions que les instruments précédents. Il n y a cependant aucun intérêt à utiliser le modèle courant, surtout destiné à la mesure de grands angles. Il en est autrement du sextant à prismes associé à une lunette fixe qui peut devenir un télémètre précis, quant à l'évaluation de l'angle.

\section{Télémètres monostatiques.}

\section{Précision à demander à l'appareil. -} Les appareils à décalage d'images présentent l'inconvénient d'exiger la connaissance d'une base, voisine de l'objet, de position à peu près déterminée, et dont la grandeur est, dans la majorité des cas, évaluée à l'estime. On s'est proposé, depuis longtemps, d'organiser un instrument qui renferme implicitement la base, laquelle deviendra une constante de l'appareil. el dont on n'aura pas à se préoccuper dans les calculs ou les lectures. 
La base, qui doit être invariable, constante, sera déterminéc par deux des points d'un système rigide; elle sera donc courte, dans les environs de $\mathrm{r}$ ou de 2 mètres, pour que le télémítre soit réalisable; pcutêtre pourra-t-on atteindre 4 ou 5 nètres. A chacune de ses extrémités, un système optique produira une image; un opérateur unique examinera la position relative des deux images.

Les syslèmes opliques seront équivalents quant à leurs effets; la base placée de façon que les faisceaux incidents, en atteignant les extrémités, tombent symétriquement, ou à peu pris, sur elle. L.e triangle á résoudre sera donc, étant donnée la faiblesse admise de la base par rapport à la distance, isoscèle ou rectangle. La position des images évaluera la parallaxe.

Gémétriquement, donc, on aura la relation :

$$
p=\frac{b}{\gamma} ;
$$

d'où, en supposant la base constante:

$$
\begin{gathered}
\frac{\delta p}{p}=\frac{b}{p \gamma^{2}} z_{\gamma}=\frac{p}{b} z_{\gamma}, \\
\delta p=\frac{p^{2}}{b} \delta \gamma .
\end{gathered}
$$

En évaluant jy en secondes, cette équation donne:

$$
\left(\grave{z}_{i}\right)=200000 \frac{b \Sigma p}{p^{2}} .
$$

On anra une idée de la précisionà exigerdans les mesures angulaires, - car tout télémètre est un goniomètre, en supposant qu'avec une base de a mètres, on se propose d'évaluer à 50 mètres près, comme erreur maxi- 
mum pratique, une distance de 2000 mètres. La formule ci-dessus donine $(\partial y)=5^{\prime \prime}$. C'est la plus grande erreur qu'on puisse se permettre sur la mesure de la parallaxe.

\section{Télémètre à lunettes parallèles. - Si} deux lunettes à réticule, identiques, $L_{\text {, et }} \mathrm{L}^{\prime}$, sont disposées de façon que leurs axes optiques soient parallèles, et si la première est dirigée sur un point éloigné $\mathrm{A}$, dont limage viendra se placer sur le centre du réticule, l'image de A dans la lunette $L^{\prime}$. se trouvera à une distance $\eta$ de la croisée des fils du réticule correspondant; $r_{\text {i }}$ est compté parallèlement a la base $b$, intervalle des axes optiques. La distance cherchée $p$ est alors donnée par la relation

$$
p=\frac{b f}{n} .
$$

On évaluera $\eta_{\text {en }}$ adjoignant au rélicule de $\mathrm{L}^{\prime}$ un micromètre sur glace ou à fils, et alors $\mathrm{L}^{\prime}$ agira comme unc lunette stadimétrique, le système des deux lunettes n'ayant d'autre but que de constituer et de porter en avant une base $b$, dont le point visé est l'une des extrémités. On pourra encore évaluer $\eta_{i}$ en mesurant le déplacement angulaire $\alpha$, qui est nécessaire pour assurer la coïncidence, dans la lunette $L$ ', de l'image et du centre du réticule, quand $L^{\prime}$ tourne autour du centre optique de son objectif. Dans ce dernier cas, $n=f x$, et par suite:

$$
p=\frac{b}{\alpha} .
$$

Sous cette dernière forme, le télémètre à deux lunettes a été proposé un peu avant ${ }_{1}{ }_{7} 3$ par de Gen- 
sanne ${ }^{1}$, et présenté à l'Académie des Sciences de Paris. Les rapporteurs de la proposition émirent des doutes sur la précision de l'apparcil.

L'idée a été reprise vers 1877 , par le général américain Berdain (59), dont le télémètre est identique à l'appareil qui vient d'ètre cité, à cela près que la base est horizontale, et que l'ensemble est plus complètement ćtudié au point de vue mécanique.

A la même époque, le colonel autrichien Roskiewica (59) faisait construire un télémètre du même genre, mais à lunettes fixes. l'une d'elles comportant un réticule à fil mobile.

Ces appareils n'ont donné de résultats satisfaisants qu'immédiatement après leur réglage, lequel exige l'abservation préalable soit d'un point situé à une distance parfaitement comnue, soit d'un point Iumineux à l'infini (un astre, en principe).

En admettant les caractéristiques du télémètre considéré au $\$ 255$, et remarquant que $14 \mathrm{mploi}$ de l'instrument comporte deux visées et, par suite, une précision de pointé de $2^{\prime \prime}, 5$, on voit que l'angle de rotation de la lunette doit être évalué avec cette précision. Avec le télémètre Roskiewic\%, le déplacement du fil réticulaire devrait être mesuré avec une précision de $\frac{2,5}{200000} f=0^{\mathrm{mm}}, 08$, en faisant $f=7^{\text {oo }}$ millimètres, valeur convenable pour la focale d'un objectif devant être ouvert à 55 millimètres au moins; en vuc de son pouvoir séparateur; la lunette reste d'ailleurs

1 De Gessavxe. Machine pour mesurer d'une senle station de petites distances inaccessibles. Machines et inventions approuves par l'Académie royale des Soiences de Paris, t. VII, 1737.

IRIS - LILLIAD - Université Lille 1 
suffisamment courte. Facile à atteindre dans un instrument de laboratoire, cette précision se conserve rarenient au dehors.

258. Télémètres à superposition d'images. - Base variable. - In autre inconvénient des télémètres à deux lunettes est de nécessiter soit des observations successives, quoique a intervalles rapprochés, par un même opérateur, soit l'emploi de deux observateurs. On a lenté d'éviter ce défaut en transportant optiquement, dans le voisinage l'une de l'autre, les images objectives de manière qu'elles puissent être observées simultanément par un mênue opérateur. De leur position relative ou de la ríformation a faire subir à l'instrument pour assurer la superposition des images, on déduira la parallaxe du but.

Le plus ancien instrument de ce gence paraît être

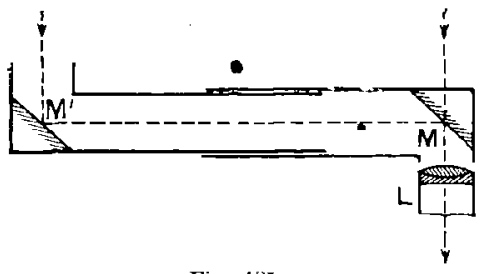

Fig. 135. celui construit par Magellan (59) en $177^{5}$ (lig. I 35$)$ : deun miroirs situés à une distance variable $b$ font entre eux un tries petit. angle $\%$; une lunette L. recoit à la fois limage directe (le miroir $M$ ne couvrant que la noitié de l'objectif) et Yimage doublement réfléchie par $\mathbf{M}^{\prime}$ et M. En faisant varier la distance des mïroirs, la superposition des images peut être obtenue, et on a alors:

$$
p=\frac{b}{\sin 2 y}=k b .
$$


Ce dispositif, plus ou moins perfectionné, a été repris depuis par Salncuve vers $\mathrm{I} 867 ;$ il se rencontre dans les télénic̀tres plus récents proposés par M. Aragno ${ }^{1}$, M. Neilson ${ }^{2}$, M.M. Lawford, Capper et Beck ${ }^{3}$.

La difficulté à vaincre dans la réalisation de ce principe est le maintien de la constance, à un petit nombre de secondes près, de l'angle $\because$ des axes des lunettes, pendant que la hase varie.

259. Base invariable. - Dans la plupart des monostatiques les plus récents, la base est invariable; la superposition des images est obtenue par le déplacement ou la déformation d'un organe optique qui agit sur liun des faisceaux. Ceux-ci, ajorès avoir atteint les extrémités de l'appareil, se replient, en se dirigeant l'un vers l'autre, dans la direction de la base, puis sont renvoyés dans une dircction unique, sens compris, sur un système oculaire.

Les premiers télémc̀tres de ce gence paraissent ètre ceux de Steinheil, d'Adic et de Tavernicr (59), construits un peu avant I 860 .

Leur organisation schématique est la suivante (fig. r 36) : deux miroirs plans $M$ et $\mathbf{M}^{\prime}$ sont placés aux extrémités de la base, à $45^{\circ}$ de sa direction; ils remvoient à $9^{\circ}$ de leur direction primitive et, par suite, à peu près sur la base $m m^{\prime}$ les faisceaux, issus du point $\mathrm{A}$, qui les atteignent; chaque faisceau ren. contre un objectif $0 . \mathrm{O}^{\prime}$, à proximité du miroir correspondant, puis, vers le milieu de la base, un prisme P, I' a réflexion totale. Il se forme donc deux images

1 Brevet fr. 345478 .

2 Brevet fr. 321255 .

3 Brevet fr. 3626.3. 
réelles $\mathrm{A}^{\prime}$ et $\mathrm{A}^{\prime \prime}$ à la sortie de ces prismes (la focale de l'objectif est déterminée en conséquence); ce sont ces deux images que l'oil observe par l'intermédiaire d'un oculaire $\Omega$ terrestre ou astronomique, muni au besoin d'un système déplaçant l'ensemble dęs deux images afin d'en faciliter l'observation.

Les deux prismes centranx ont leurs hypotínuses

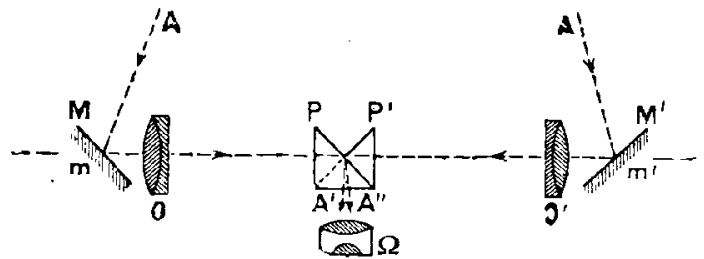

Fig. 136.

réfléchissantes respectivement parallètes aux miroirs, comme dans le téléstéréoscope d'Helmholtz; ils sont d'ailleurs l'un sur l'autre (en supposant, pour fixer les idées, la base horizontale), afin que l'écartement des images ne soit pas augrnenté de celui des centres des faces réfléchissantes, et que les faisceaux déviés par elles puissent atteindre l'oculaire sous des angles faibles avec l'axe. Il en résulte que chaque prisme ne reçoit que la moitié du faisceau tombant sur l'objectif; ceuxci ne travaillent que sur la moitié de leur ouverture, l'un sur la moitié supérieure, l'autre sur la moitié inférieure.

Si le point $\Lambda$ était à l'infini, les deux images $\mathbf{A}^{\prime}$ et $\mathrm{A}^{\prime \prime}$ coïncideraient. Quand le but est à une distance $p$, l'écartement $\eta$ de ces images ou de celles de deux 
points correspondants est lié à $p$ par la même relation que dans les télémètres à deux lunettes :

$$
p=\frac{b f}{r_{i}}
$$

car, tant que les images sont dans le champ de l'oculaire, le triangle, très allongé, est sensiblement isoscèle. La parallaxe seule intervient, et non, individuellement. les angles à la base.

La superposition des images $\mathrm{A}^{\prime}$ et $\mathrm{A}^{\prime \prime}$, d'où résultera la mesure implicite de $r_{i}$, est obtenue par un dispositif analogue au suivant, et dont le principe est de produire, entre l'un des objectifs et l'image correspondante, une déviation convenable du faisceau intéressé.

$\mathrm{Si}$, entre l'objectif et l'image réelle, et à une distance $x$ de celle-ci comptée sur le trajet du faisceau, on dispose un prisme à angle très faible $\theta$, la déviation linéaire de l'image; dans son plan, sera exprimée par $(n-\mathrm{r}) \theta x$, et la superposition de $\mathrm{A}^{\prime}$ et de $\mathrm{A}^{\prime \prime}$ sera obtenue quand on aura $\eta=(n-1) \theta x$; la distance $p$ sera donc donnée par la formule :

$$
p=\underset{(n-\mathrm{I}) \mathrm{bx}}{\mathrm{b}}=\frac{k}{x} .
$$

Comme, en général, l'organisation de l'appareil s'oppose à ce que $x$ puisse devenir nul, - le prisme déviateur devant se mouvoir entre l'objectif et les prismes centraux, - on dispose, sur le trajet de l'autre faisceau, un prisme déviatour fixe, calculé de façon que la coïncidence des images ail lieu pour des faisceaux incidents parallèles, quand $x=x_{0}, x_{0}$ étant la valeur minimum qui peut prendre la distance du prisme déviateur 
mobile à l'image. Ia formule précédente devient alors :

$$
p=x^{k}-
$$

Iorsqu'on observe avec un télémètre de ce genre un objet vertical (par exemple. un paratonnerre), la base de lonstrument étant horizontale, - se pré-

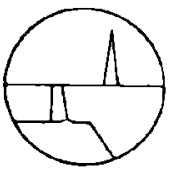

Fig. 137. sente sous l'aspect de la figure 137 ; l'image de l'objet est coupée en deux parties décalćes dans le sens horizontal; chacune d'elles est dans le demi-champ visible donmé par la moitié efficace de l'objectif correspondant, la licrne de séparation des champs pouvant être perçue si les images objectives se forment tout près des prismes centraux. Le jeu du prisme déviateur permet la réunion en une seule inlage continue des deux fragnents aperçus; la coüncidence des images est alors assurée, bien que celles-ci ne soient pas discernées dans leur ensemble. De là le nom de télérnètres à coüncidence donné à ce type d'instrumenls.

260 . Éléments optiques essentiels des télémètres monostatiques. - I.es causes qui peuvent influer sur l'esactitude des indications de l'appareil tiennent : $I^{\prime \prime}$ a la position des miroirs $M$ et $M^{\prime}$ par rapport à la hase; $2^{\circ}$ à la position relative des hypoténuses des prismes centraux; 3 "a la variation de forme de la base par suite de flexions de l'instrument; $4^{\circ}$ à la non-homngénéité des milieux traversés par les faisceaux lumineus

Cette dernière cause est commune a tous les instruments d'optique. Si elle peut ici produire un effet sen- 
sible, cela tient, malgré la faiblesse de la base, à la grande précision exigée dans la mesure des angles. Ia non-homogénéité en question est celle des couches d'air traversées. Quoique les faisceaux incidents soient toujours peu écartés, il suffit, par exemple, que l'un d'eux rase de plus près un corps plus chand que le milieu ambiant, pour qu'une déviation différente lui soit donnée, en raison de la moindre densité de la couche d'air. On perçoit cette influence dans le tremblotement continu des images quand le télémètre est à pou de hauteur au-dessus d'un sol trop échauffé.

Les erreurs dues à cette cause ne peuvent être évitées qu'en réglant les conditions el'emploi de l'appareil . son organisation optique ne peut y remédier.

En outre, au point de vue de la valeur absolue de la mesure, il est à remarquér que tout télémc̀tre ne résout pas le triangle géométrique considéré jusqüici, mais seulement celui qui est formé par la base et les tangentes issues de ses extrémités aux trajets des faisceaux lumineux qui y aboutissent. Il sera donc nécessaire de tenir compte, le cas échéant. quand la précision s'imposera, de la réfraction atmosphérique. La graduation de l'instrument ne peut être établie en conséquence que si le télémètre doit être uniquement employé dans des conditions bien déterminées.

26 r . Équerres optiques. - Les miroirs extrêmes ont été remplacés depuis longteunps par des prismes à réflexion totale. Cette substitution améliore la clarté des images; elle laisse subsister la nécessité d'une posi tion précise des organes extrêmes. Or un déplacement angulaire $\beta$ d'un prisme inflige à l'image un déplacement double $2 \beta$; comme l'appareil comporte deux 
prismes, la précision angulaire $\delta \gamma$, dans la mesure, ne sera obtenue qu'autant que l'on aura $\beta \leq \frac{T}{4} \delta \gamma$. Une telle précision $\beta$, qui est alors voisine d'une seconde, exige que les points d'appui du prisme aient des positions relatives presque invariables. Avec un prisme de 60 millimètres de côté, lo déplacement relatif des points d'appui ne doit pas être, pour

$$
\delta p=4^{\prime \prime} \text {, ou } \beta=1^{\prime \prime},
$$

supérieur à $o, 3 i$.

Cette précision se conserve difficilement; un instrument de genre exige de fréquents réglages. On les évite en remplaçant les miroirs ou prismes-miroirs par des équerres optiques.

Les équerres optiques sont des systèmes de forme invariable, qui dévient un faisceau incident, d'un angle constant, en principe égal à $90^{\circ}$, en pratique très voisin d'un droit, et cela quelle que soit l'incidence sous laquelle le faisceau est reçu. Une condition supplémentaire doit être ajoutée, sans laquelle l'équerre optique est impossible à réaliser : le, faisceau doit rester normal

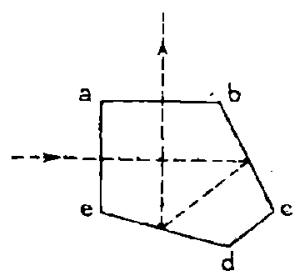

Fig. 138. à une direction déterminée.

Une équerre optique simple est constituéc par le prisme en verre de la figure $\mathbf{3} 38$; les deux faces $b c$ et $d e$ sont argentées et se coupent à $45^{\circ}$; l'angle $a$ est droit; les angles $b$ et $c$ valent $\times 35^{\circ}$; la face $c d$ n'a aucune action optique, elle supprime uniquement l'angle aigu du prisme. Un faiscean tombant sur 
ae sort en émergeant de $a b$, les directions faisant un angle de $90^{\circ}+30$ si l'angle des faces $b c$ et $d e$ est de $45^{\circ}+0$. La propriété subsiste, quelle que soit l'iucidence du faisceau sur la face d'entrće, pourvu que les rayons restent normaux à l'intersection des faces réfléchissantes $b c$ et de. C'est l'équerre oplique cmployéc par le capitaine Gautier, en 1865 , dans son télémètre instantanc; par le colonel Goulier, en 1863 , dans le nautomètre à prismes et dans les appareils de son télémètre. C'est actuellement l'équerre la plus employée, malgró l'absorption lumineuse due aux miroirs, parce qu'elle est la plus simple.

La combinaison prismatique Porro, à laquelle on retranche un demi-prisme, constitue une équerre optique à trois réflexions totales, - et par suite sans argenture, - dont l'axe (direction à laquelle les faisceaux doivent rester normaux) est l'intersection des faces d'entrée et de sortic. Celle équerre a élé mentionnée dans les brevets ${ }^{1}$ rclatifs au télémètre Barr et Stroud. Les difficultés de travail ne permettent pas de la taillez dans an seul bloc de verre, car les faces polies ne forment pas un polyèdre convexe; on doit se borner à coller an baume un prisme et un demi-prisme. On ne peut alors répondre de l'invariabilité du système.

On a constitué des équerres optiques en assemblant des miroirs. La solution est défectueuse; les appuis ne sont jamais suffisamment assurés.

La nécessité d'avoir le faisceau incident perpendiculaire à une certaine direction fixe de l'équerre optique n'est pas un inconvénient. En effet, la variation d'inci-

1 Brevets français $199368,239528,317896,333047,338670$, 376711 .

IRIS - LILLIAD - Université Lille 1 
dence du faisceau peut toujours être attribuée au déplacement du prisme-équerre; si celni-ci tourne autour de son axe, l'imagre reste fixe; s'il tourne autour d'une autre droite, l'image nonte ou descend dans le champ de l'oculairc. On s'en rend compte par l'aspect de l'image totale qui présente alors des points reproduits dans les deux champs ou dans laquelle des parties sont éliminées (image allongée ou raccourcie); il suffit de modifier en conséquence l'orientation du prisme. Quand l'image est complete et sans points doublés, la condition nécessaire à l'emploi de l'équerre est réalisée. L'axe de l'équerre est disposé normalement au triangle à résoudre; la rotation du prisme autour d'une perpendiculaire à cet axe rst alors corriggé comme il vient d'être dil.

262. Système optique central. - Les prismes centraux sont cimentés l'un sur l'autre. Une rotation

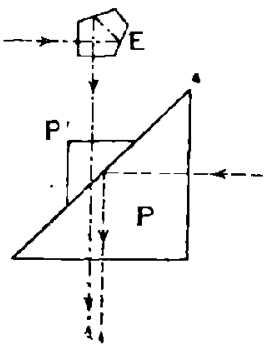

Hig. 139. de leur ensemble n'influe pas sur la position relative des deu $x$ images: elles se déplacent de la même quanlité et dans le mème sens. Mais ce collage en porte-à-faux est assez délicat, et on ne peut cependant compter que sur lui pour assurer l'invariabilité du système.

La combinaison suivante ${ }^{1}$ (fig. 139), indiquée par MM. Barr et Stroud, pent remplacer le double prisme central; elle comprend deux prismes $\mathrm{P}^{\prime}, \mathrm{P}^{\prime}$, de

1 Un dispositif analogue est employé par la maison Zeiss. (Brevet fr. 36:740.) 
dimensions différentes, collés au baume de Canada par leurs faces hypoténuses, et par suite sans porte-à-faux. La marche des rayons est suffisamment indiquée dans la figure; les uns traversent la partie centrale des deux prismes, agissant alors comme lame à faces parallèles ; les autres subissent, en dehors de la région collée, la réflexion totale ordinaire. Cotte disposition détruil la symétrie primitive du télémètre et exige l'emploi d'une équerre optique intéricure É ramenant l'un des faisceaux perpendiculairement à l'autre.

Les mêmes constructeurs emploient, dinns un de leurs types de télémètres, comme système central, un prisme à réflexion totale dit séparateur, dont une face est taillée suivant un angle très obtus. L'instrument est constitué alors de manière à superposer d'abord en direction el sens les faisceaux tombant sur les extrémités de la hase; ces faisceaux sont séparés ensuite par le prisme oblus, ‘ui en outre les réfléchit ver's l'oculaire dans une direction déterminée. Ce prisme séparateur est aussi employé dans d'autres instruments des mèmes - opticiens; il est joint au système central ordinaire ou à des prismes redresseurs d'images. Son but n'est alors que de fixer la ligne de séparation des deux champs.

263. Flexion de la monture. - La flexion de l'instrument est l'un des points les plus délicats de la construction d'un télémètre monostatique. Cette flexion peut tenir au poids même de l'appareil, aux secousses qui lui sont infligées, à la dilatation de la monture. Si l'axe de l'apparcil fléchit de façon à prendre une courbure circulaire de flèche $\varepsilon$ par rapport à la base primilive $b$, la déviation relative $o$ des faisceaux, égale Instruments optiques. 
à celle des tangentes extrêmes, a pour expression approchée :

$$
\varphi=\frac{4 \equiv}{b} \text {. }
$$

Elle doit être de l'ordre de la seconde pour que son influence soit insensible. Pour $b=2$ mètres et $\varphi=I^{\prime \prime}$, on aurait $\varepsilon=2,5 ;$. . Même en diminuant la précision, on ne saurait tolérer une valeur bien considérable de la flèche.

Le maintien de la rigidité de l'instrument est d'ordre mécanique. Yous nous bornerons à signaler ici que, dans le télémètre Barr et Stroud, les éléments optiques (à l'exception des équerres optiques de base) sont assemblés dans une monture qui est protégée par une enveloppe extérieure, à laquelle la monture est reliée par des articulations dérivant du joint à la Cardan, de manière à assurer une certaine indépendance entre les deux corps du télémètre.

Dans le nautomètre à prismes du colonel Goulier (59) et dans le télémètre Wild (brevet fr. 350963), un objectif unique reçoit les deux faisceaux. La flexion n'a. aucune influence. Mais cette disposition oblige à donner de grandes dimensions aux éléments rélléchissants.

264. Organe déviateur. - L'emploi du prisme déviateur mobile le long de l'axe est le mode le plus commun pour produire la superposilion des images. Bien que la relation qui lie la distance au déplacement du prisme se présente sous la forme hyperbolique $p\left(x-x_{\mathrm{o}}\right)=k$, l'amplitude du déplacement total est asser grande pour satisfaire aux exigences de la pratique. Le déplacement $x-x_{\circ}$ peut être lu directement sur une graduation 
linéaire tracée en conséquence, que celle-ci se déplace devant un index fixe ou inversement; le cas est assez fréquent. Dans des spécimens de télémètres Barr et Stroud, la lecture s'opère sur le long d'une hélice cylindrique qui amplific le mouvement du prisme ou sur une graduation tracée sur glace reliće au prisme, que l'on observe, far un second oculaire, à l'aide de l'oil non occupé par les images.

Dans ses télémètres, Gaulier (33) employait une lame épaisse à faces parallèles, mobile autour d'un axe normal à celui de l'instrument. Le déplacement linéaire ainsi produit, quand la normale à la lame fait avec le faisceau incident sur elle un angle $\theta$, est :

$$
\begin{aligned}
& n-e \frac{\sin (\theta-r)}{\cos r}, \\
& \sin r=\frac{\pi}{n} \sin \theta,
\end{aligned}
$$

avec :

$e$ étant l'épaisseur de la glace.

Goulier (59), dans son nautomètre, utilisait le même système déviateur à lentille que dans le télémètre.

Le prisme biréfringrent de Rochon peut remplacer, sans avantage d'ailleurs, le prisme déviateur simple. La lentille fendue de Dollond est susceptible d'application quand les faisceaux ont été ramenés dans lo mnème sens.

265. Télémètre stéréoscopique. - Un autre procédé, qui ressortit à l'optique physiologique, a été employé pour assurer la superposition des images. L'instrument est alors identique, en principe, au téléstéréoscope.

Voici comment on pent concevoir la transformation 
de ce dernier instrument en télémètre, telle que l'a réalisée la maison Zeiss.

Imaginons l'appareil d'Helmholtz: dans le paysage, un sentier tracé en lacets sur un plan incliné, et, sur son parcours. des bornes, hectométriques, par exemple, dont la chiffraison indique leur distance à l'instrument. Supposons deux plaques sensibles dans les plans focaux des objectifs, et admettons que, ces plaques restant à demeure, le sentier puisse ètre photographié, les images développées et fixées, et que dans les clichés défnitifs il ne reste plus que les bornes et la chiffraison. Si maintenant on dirige l'appareil de manière à faire apparaître dans le champ un objet situé à $q$ hectomètres, on percevra une différence de parallaxe quand on passera de la coïncidence des images du but à la coüncidence de deux bornes dont le numéro commun est trop différent de $q$. On amèncra, par tâtonnements méthodiques, la double image superposće du but à proximité d'une borne $q$ ', dont les images photographiques paraîtront en coüncidence, en même temps que celles du but, sans qu'on nerçoive une variation de convergence visuelle. La loi d'Helmholtz étant admise, la formule établie antérieurement donne :

$$
\bmod \left(q^{\prime}-q\right) \leq \frac{q^{\prime 2}}{200 \operatorname{Cim}-\overline{q^{\prime}}} .
$$

La distance du but est donc $q^{\prime}$, arec une erreur possible expriméc par le second membre de cette inécralité.

Dans le télémètre stéréoscopique \%eiss. les bornes en question sont figurées par des virgules sur les plaques micrométriques établies d'après les données géomètriques; un des spécimens comporte pour $G$ et $m$ des. 
valeurs égales entre elles et voisines de 23 ; le coefficient constant du dónominateur vaut roo kilonètres; c'est l'infini de l'appareil. Il devrait donner à 40 mètres près la distance de 2000 mitres; à roo mètres. celle de 3 ooo mètres.

L'organisation pratique de ce télémètre cst du même ordre que celle des monostatiques ${ }^{1}$ : équerres optiques, etc. ; les causes d'erreur sont analogues; sinon identiques. On peut y ajouter celles qui tiennent au déplacement possible, si faible soit-il, des inicromètreş.

Chaque micromètre peut ne comporter que l'image d'un seul petil objet, un ballon, par exemple. Suivant l'intervalle linéaire qui séparera ces deux images, leur coïncidence s'établira simultanément avec celle des images d'un objet du champ; tant que cet écartement ne répondra pas à la distance du but, on sentira le ballon flotter dans l'espace quand on passera de son observation à celle de l'ohjet. Une vis micrométrique. mesurera l'intervalle convenable à la stabilité de la perception; la valeur de la distance cherchée s'ensuivra. Le procédé a été indiqué, croyons-nous, par le constructeur anglais M. Forbes; nous ignorons si l'instrument a été réalisé.

266. Appréciation des télémètres monostatiques. - La conservation du réglage, c'est-à-dire le maintien du degré très élevé de précision qu'on peut atteindre dans un télémètre monostatique, a été jusqu'ici la pierre d'achoppement dans' leur utilisation. L'emploi d'équerres à prismes, le transport des objectifs vers le centre de la base, où ils peuvent être réunis

1 Société Carl Zeiss. Brevet fr. 375682.

IRIS - LILLIAD - Université Lille 1 
en un seul, ont apporté rles améliorations indiscutables au type primitif. Mais ces perfectionnements n'ont pas toujours assuré la constance des indications.

Lorsqu'on dispose de temps et d'espace, on peut vérifier, d'une façon suffisante, le réglage d'un télémètre en s'assurant que sa graduation marque l'infini quand on vise un astre; si celle condition n'est pas réalisée, il suffira généralement de déplacer en conséquence l'origine de la graduation pour que les visées sur d'autres buts conduisent-à des mesures exactes. A cet effet, - comme aussi pour la viscée sur points lumineux, tels que phares, feux de navires, etc., MM. Barr et Stroud interposent, en temps utile, une lentille cylindrique dite anastigmatiseur entre l'oculaire et les images objectives; l'image se transforme en une ligne droite parallèle à l'axe du cylindre; elle peut donc déborder la ligne de séparation des champs.

La visée sur un astre est, en plein jour, un cas exceptionnel. Si le télémètre est disposé à poste fixe, comme il le serait dans une batterie de côte, - on aura généralement des points de repère suffisamment éloignés et dont la distance sera parfaitement connue, qui permettront la vérification de l'instrument. Pour un appareil mobile, si d'ailleurs le temps ne fait pas défaut, on pourra utiliser le procédé ${ }^{1}$ du colonel Souchier, qui consiste à vérifier l'infini à l'aide d'une mire, égale à la base, placée à 25 mètres environ de celle-ci et parallèlement à elle: les deux lignes de foi qui limitent la longueur utile de la mire doivent se superposer; les points de la mire à faire coïncider

l Employé dẻjà dans le télémètre Roskiewicz (59).

IRIS - LILLIAD - Université Lille 1 
doivent être déterminés avec une précision linéaire au moins égrale à $o^{\mathrm{mm}}, \mathbf{1} 25 \pi$, si la précision angulaire du télémètre est de $\varpi$ secondes.

Ces genres de réglage ne permettent pas toujours de conserver aux monostatiques la dénomination de télémètres instantanés, qu'on leur accorde généralement. On a essayé d'adjoindre à l'appareil les organes qui permettraient un réglage rapide sans observation d'objet extérieur. On a eu recours au procédé de la collimation employé dans l'étude de la flexion des lunettes astronomiques; les télémètres précités de Berdan et de Roskiewicz comportent des lunettes collimatrices spéciales. Le Cyre, qui a construit de nombreux spécimens de monostatiques, a fait intervenir (59) la collimation sous toutes ses formes, et, en outre, le principe du retournement, mis constamment en ocurre dans le réglage des appareils topographiques. Mais, en général, la difficulté n'est que reculée: Ia conservation du réglage doit alors être réalisée dans les organes accessoires; l'examen des dispositions de détail met cette conclusion en évidence.

Le problème ne paraît cependant pas insoluble. En adoptant rigourcusement la móthode de collimation astronomique, le réglage scrait possible; mais les dimensions de l'instrument, dans le sens de la base, devraient être au moins doublées. Le mode d'emploi du télémètre pcut faire rejeler a priori cette condition. ${ }_{26} 6$. Emploi des télémètres monostatiques. - L'utilisation des télémètres ressort des propriétés qui ont été indiquées pour chaque système. Plus la base sera étendue, moins les mesures angulaires pourront être précises; mais alors il y a lieu de tenir 
compte de la possibilité de commettre des erreurs, des fautes même, sur le point de viséc. La méthode exige des buts fixes, bien définis, du temps et du calme de la part de l'opérateur.

Les télémètres à grande base, résolvant un triangle rectangle, demandent en outre un espace convenable; il faut que la base puisse s'étendre sur un terrain sensiblement horizontal, et dans une direction bien déterminée.

Les appareils qui nécessitent l'observation d'une base à hauteur du but sont très précis quand cette base est une mire topographique. Quand on ne possède sur elle que des renseignements incertains, le télémètre ne peút avoir d'autre objet que d'éviter les erreurs grossières que l'on peut commcttre sur l'appréciation des distances dépassant quelques centaines de mètres.

Les télémètres monostatiques sont des instruments remarquables quand leur réglage se conserve. Mais quand celui-ci n'est pas assuré, des erreurs invraisenblables peuvent être commises; car il faut peu de chose pour produire dans l'un des faisceaux une déviation de quelques secondes.

Pour les télémètres stéréoscopiques, il faut obscrver que leur théorie repose sur la loi d'Helmholtz, qui n'a pas été suffisamment vérifiée. Mais, fût-elle exacte, alr moins pour des observateurs choisis, il est indéniable que l'état physiologique de l'individu peut faire varier rapidement, et sans que celui-ci s'en doute, sa faculté de perception de la profondeur. Cette propriété que possède la vision binoculaire de sentir la variation de convergence des lignes visuelles est soumise à des 
modifications plus importantes et moins visibles que la propriété, pour un ceil unique, de percevoir, par la coïncidence des images, l'identité de deux directions. Ingénieux dans son principe, le télémètre stéréoscopique le cédera toujours an monostatique ordinaire.

\section{Mesure de petits intervalles.}

268. - Le microscope se prête naturellement à la mesure d'intervalles séparant deux points ou deux traits situés sur un plan ou sur une arête de surface réglée. A cet effet, à l'oculaire négatif habituel est substitué un oculaire du même type, mais à verres mobiles, et portant entre ces derniers une plaque micrométrique.

Quand il n'y a pas intérêt à utiliser un champ étendu, et que le microscope est destiné uniquement à des mesures, il y a avantage à le munir d'un oculaire de Ramsden, et à laisser le micromètre fixe. Celui-ci sera soit une glace graduée, soit mieux un micromètre à fil mohile.

La précision de ces mesurcs au microscople peut êlre considérable.

Si l'objectif ne peut être suffisamment approché de l'objet, on pourra opérer sur son image, produite à peu près en vraie grandeur par une lentille convergente de faible focale.

Un autre procédé, utilisé dans l'ophtalmoscope d'Helmholtz, consiste à employer une lunette à réticule de laboratoire, qui est identique à un microscope à très long foyer, dont l'objectif porte en avant de lui 
une glace parallèle épaisse. En amenant successivement les deux extrémités de l'intervalle en coïncidence avec le réticule, par simple jeu de la lame, la variation angulaire de celle-ci détermine la valeur de l'intervalle en question.

Tout appareil de mesure d'angles peut être transformé, plus ou moins efficacement, pour la mesure de petits intervalles. Nous signalerons en particulier l'emploi du prisme de Wollaston, utilisé par le capitaine Lafay dans la mesure des écrasements des crushers".

1 Cangrès internat. des méthodes d'essai des materiaux de construction. Paris, 1900. 


\section{CHAPITRE XV}

LYSTRUMENTS BASÉS SUR LE PRINGIPE DE L'ALTO-COLLYMATION

269. Principe de l'auto-collimation. - Le principe de l'auto-collimation peut être énoncé sous la forme générale suivante: si (fig. I/10) un faisceau lumineux, issu d'un point $P$, tombe, après passage à travers un système optique $\mathrm{S}$ quelconque, sur une surface réfléchissante M, de façon que tous ses rayons soient alors

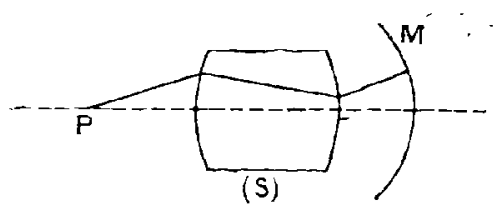

Irig. 140. normaux à cette surface, le faisceau reviendra en arrière en suivant un parcours exactement contraire, et formera une image réelle qui coïncidera avec $P$.

La surface M peut d'ailleurs être l'une des faces d'un élément réfringent, car il existe toujours un faisceau réfléchi ; il suffira, pour l'uliliser, d'augmenter convenablement l'intensité de la lumière incidente.

Le procédé de l'auto-collimation a été utilisé par Foucault, - avec les variantes nécessaires, - pour 
contrôler, par la pureté de l'image formée, la forme de la surface $\mathrm{M}$, lorsque celle-ci doit ètre normale à un faisceau donné. Ad. Martin (66) s'en est servi pour examiner les défauts et mesurer les indices des verres optiquement taillés.

Dans un certain nombre d'applications, la surface M qui produit l'auto-collimation est un miroir plan; le système optique $S$ transforme on faisceau parallele le faisceau divergent issu de $P$. Dans ces conditions, la coüncidence de $\mathbf{P}$ avec sor image assure à la fois la position du point $P$ à l'un des foyers du système $S$ et l'orientation du miroir $\mathrm{M}$ perpendiculairement à l'axe optique du même système.

Quand le miroir $M$ se déverse d'un très petit angle sur l'axe optique, l'image produite, tout en restant suffisaminent nette dans le plan focal de $\mathbf{S}$, cesse de coüncider avec P. De la mesure de l'écartement linéaire ainsi produit, on peut déduire la variation angulaire du miroir; car si le miroir tourne d'un angle $\theta$, l'augle entre le faisceau incident direct el le faisceau rétrograde est $2 \%$; l'écartement linéaire $r_{1}$ a pour valeur $\eta=2 f 0, f$ étant la focale du système interposé.

Lintervalle $r$ est indépendant de la distance du miroir au système optique $\mathrm{S}$.

270. Oculaires pour auto-collimation. Les physiciens ulilisent le principe si délicat de l'autocollimation sur miroir plan pour disposer les faisceaux parallèles dans des directions bien déterminées; par exemple, dans la mesure des angles des prismes avec le groniomètre de Babinet, pour placer l'axe optique de la lunette (qui a la direction du faisceau reçu) perpendiculairement à l'axe de rotation de l'appareil et 
INSTRUMENTS BASÉS SUR LE PRINCIPE DE LAUTO-COLLIM. 361 aux faces des prismes; de même avec les spectroscopes et spectromètres, etc. Dans ce cas, l'objet $P$ est constitué par le réticule à fils d'araignée, muni de l'oculaire éclaireur Cornu. Quand la glace (ou face du prisme) est sensiblement perpendiculaire à l'axe optique, on perçoit dans le champ de la lunette un second réticule, image à intensité faible du réticule récl. En effectuant la superposition des images fournies par l'oculaire, l'axe optique et celui du miroir sont amenés au parallélisme.

L'emploi, comme objets lumineux, de réticules a fils n'est pratique que lorsque le plan d'auto-collimation n'est pas trop éloigné de l'objectif (a à 3 mètres, au maximum), sinon l'image est à peine visible; en outre, en raison de la présence de la glace éclairante, l'emploi d'oculaires à court foyer est impossible. Dans ses ingrénieux appareils pour le contrôle des surfaces optiques, M. Laurent (62) utilise, comme objet lumineux (fig. I/4), une tròs petite ouverture circulaire $o$ ( de $\mathrm{o}^{\mathrm{mm}}, 3 \mathrm{de}$ diamètre), fortement éclairée par l'intermédiaire d'un très petit prisme $p$ à réflexion totale. I'image ne peut plus être placée en coïncidence avec l'objet, puisque celui-ci est caché à l'cil; elle est amenée tangentielle-

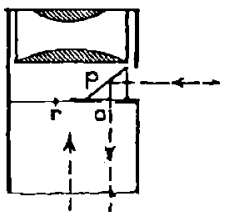

Fig. 141. ment aux deux fils d'un réticule $r$. L'axe optique fait alors un angle fixe, non nul, avec la normale au miroir auto-collimateur. Cette circonstance ne présente aucun inconvénient, car il s'agit de mesures différentielles.

27 I. Vérification des droites et des surfaces planes étendues. - Si, devant une lunette auto-

Instruments optiques. 
collimatrice fixe, se déplace le support auquel est invariablement relié le miroir de collimation, l'angle accusé par le déplacement de l'image sera un des éléments qui déterminera la position, par rapport à l'axe optique, de la droite qui joint les deux stations du miroir; l'autre élément sera la distance de ces deux stations. Le plus ou moins d'éloignement du miroir à la lunetto n’interviendra pas dans les mesures.

Sur ces propriétés, et gråce à la sensibilité de la méthode, le commandant Dévé (16) a établi une série d'appareils destinés au contrôle de la rectitude et de la planéité des organes de machines, à la vérification des barres et des trous cylindriques allongés, à la mesure des faibles courbures (ou arcure) de ces cylindres.

L'un d'eux est utilisé pour la vérification du dressage des canons de fusil, pour lequel l'ouvrier emploie déjà un procédé optique simple, mais demandant une longue pratique; Ies défauts de rectitude de l'axe lui sont, en effet, révélés par les déformations ou l'aspect de l'image anamorphique que la surface cylindrique intérieure du canon donne d'une droite lumineuse.

L’appareil Dévé (fig. I 42) comporte une lunette L à

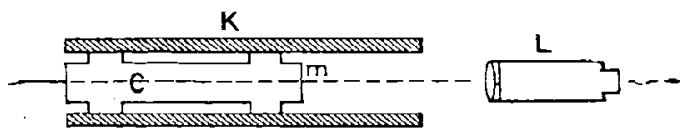

Fig- 142.

réticule à fils, fixe ; à l'intérieur du canon $\mathbf{K}$, également fixe, se déplace un calibre $C$, cylindre en acier, dont la base $m$, tournée du côté de l'objectif, est polie spécu- 
INSTRUMENTS RASES SLR IE PRINCIPE DE L'AUTO-COLLIM. 363 lairement; deux portées du calibre sont appuyées contre. la paroi par des segrments sciés formant ressorts. Si l'axe du canon est horizontal, ainsi qu'un des fils du réticule, et si on fait déplacer le calibre sams lui imprimer de rotation, les déplacements du fil horizontal du réticule mesureront les inclinaisons successives de la génératrice, plus on moins déformée, parcourue; ceux du fil verlical évalueront ses déviations par rapport à un plan vertical.

Le mème principe, à l'aide d'autres organes, est appliqué aux autres appareils. I a lunette est agencée de façon à avoir un champ plus étendu que ne le comportent les instruments déjà énumérés. Dans ce but, en avant de l'oculaire est placé (fig. I 43 ) un prisme $\mathbf{P}$ d̀ lame d'air (lame de $\mathrm{O}^{\text {mm }}$, I d'épaisseur, dont les faces font entre elles un très petit angle) qui lásse voir à travers lui, par l'oculaire. La lame donne, d'une ouverture circulaire $\omega$ (trou entouré d'un anneau), deux images superposées dans le plan focal de l'objectif, et dont le diamètre com-

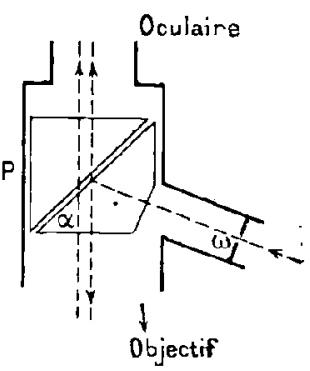

Fig. 143. mun est de a millimetre. Cette double image joue le mème rôle que la petite ouverture de l'oculaire de Laurent; l'oeil ne la perçoit pas; il ne reçoit que l'image renvoyée par le miroir. Quant au prisme, il est calculé de fáçon que l'intensité de cette dernière image soit maximum; cette condition conduit à donner à l'angle a la valeur de $35^{\circ}$, qui est, sauf une légère correction, cclle déterminée par la for- 
mule de Fresnel. La lunette est dite auto-collimatrice d longue portée.

La disposition de ]'organe de collimation varie avec le rôle de l'appareil. S'il s'agit de vérifier une ligne droite ou un plan (marbre d'atelier, par exemple; sa vérification revient à celle d'un certain nombre de droites méthodiquement tracées sur lui), on utilise un miroir porté sur un chariot à picds étroits; l'écartement de ces derniers peut ètre fixe ou variable. Le chariot est successivement conduit le long de la ligne à vérifier, de façon que les pieds postérieurs prennent exactement la place des pieds antérieurs, ou vice versa; à chaque station, l'image perçue dans la lunette est centréc sur le réticule. Les organes qui produisent le déplaccment nécessaire évaluent l'angle des doux positions du miroir, et par suite la pente du côté du cheminement.

La précision des mesures peut ètre doublée en remplaçant le mịroir par un prisme $\mathbf{P}$ à réflexion totale, et

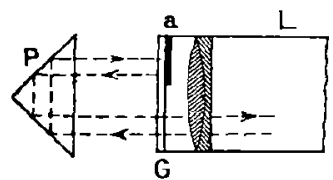

Fig. 1/4. en adjoignant à lá lunette $L$ une glace $G$ dont une faible région $a$ est argentée. La figure 144 montre cette disposition of indique la marche des rayons.

Pour la vérification des barres prismatiques ou cylindriques, le chariot ordinaire à miroir est disposé sur un support, dil équerre à trois branches, qui s'interpose entre lui et la barre, et qui comporte des surfaces d'appui rigoureusement réglées. En outre, dans le chariot, le miroir de collimation est rendu exactement normal aux lignes d'appui, par comparaison avec un 
INSTRLMENTS BASÉS SUR LE PRINCIPE DE L'AUTO-COLLIM. 365 chariot de réglage qui porte, au lieu de miroir, une glace d̀ faces parallèles; celle-ci est réglée normalement aux appuis par la méthode de retournement.

Les mêmes dispositions sont prises, à la forme près des appareils, pour la vérification des trous cylindriques.

Quand il s'agit de mesurer les variations de diamètre de l'âme cylindrique d'une bouche à feu, l'appareil entier porte le nom d'étoile mobile optique. Le chariot est alors cylindrique; ses pieds ne servent qu'à le faire porter sur la surface intérieure du canon. A l'avant, deux glaces coupées en forme de secteur à $120^{\circ}$ sont montées sur les petits bras de leviers dont les grands bras reposent respectivement sur chacun de deux pistons ou touches diamétralement opposés. L'angle des miroirs, traduit dans la lunette par l'écartement des images qu'ils donnent, est proportionnel à la somme des saillies des touches, comptées à partir du diamètre de réglage, qui produit des images superposées, les miroirs étant alors parallèles. On peut obtenir ainsi les variations successives des diamètres, suivant un parcours déterminé, génératrice ou rayure. L'espace laissć libre par les deux miroirs est rempli par un troisième, dont l'image correspondante sert à évaluer l'arcure. La confusion des images est évitée par la coloration différente des trois miroirs, qui sont blanc (glace ordinaire), vert et rose.

En dehors des usages indiqués, les appareils se prêtent. à la vérification du parallélisme et de la perpendicularité des droites et des plans, quelles que soient, dans la majorité des circonstances, les conditions qui définissent ces éléments. 
366 ORGANISATION ET EHPLOI DES INSTRUMENTS

Ces instruments sont évidemment d'un maniement délicat; mais on n'a jamais obtenu de mesures à un petit nombre de microns près qu'au prix de quelques efforts; à moins qu'on ne tienne pour de la précision l'obtention de nombres comportant beaucoup de décimales, sans s'occuper du rang à partir, duquel elles sont inexactes. 


\section{INDEX BIBLIOGRAPHIQUE}

1. ABbE (E.).

2. -

3. Alembert (d').

4. André (Ch.).

5. Banbotte

6. Batluaun (B.).

7. Berthelot (D.).

8. Biot (J.-B.).

9. -
1. Beiträgezur Theorie des Mikroskops und der mikroskopischen Wahrnehmung. Archiv für Mikroskopische Anatomie, herg. von Max. Schultze. Bonn, t. IX, 1873.

2. Ueber die Verwendung des Fluorits für optische Zwecke. Zeit.f. Instk., t. X, 1890.

Mémoire sur les moyens de perfectionner les lunetles. Histoire et Mémoires de l'Academie des Soiences de Paris, 17641765-1767; et Opuscules mathèmatiques (principalement le t. III).

Etude sur la diffraction dans les instruments d'optique (thèse). Annales scientifiques de l'Ecole normale supérieure, $2^{\circ}$ série, t. $V, 1876$.

et Rossin. Note sur un oculaire astronomique polyalde. $C$. $R$, Académie Sc, t. XXVI, 1818.

Conrs d'Astronomie, à l'usage des étudiants de la Faculté des Sciences, 1893, 1er volume. Paris, Gauthier-Villars.

Sur une méthode optique nouvelle pour la mesure des températures en valeur absolue. Ann. de Ch. et Phys., 7e série, t. XXVI, 1902.

1. Traité élémentaire d'Astronomie physique, $3^{\mathrm{e}}$ édition, $1841-45$ (10r et $2^{\mathrm{g}}$ volumes ).

2. Mémoire sur les lunettes achromatiques d̀ oculaires multiples. Mém. de $l$ 'Ac. royale des Sc. (nouvelle collection), t. XIX, 1845. 
10. Bruvs (H.).

11. Charey (G.).

12. Chimant.

13. Cazapski (S.).

14. Daguin (P.).

15. Daubresge $(\Lambda$.$) .$

16. Dévé (Ch.).

17.

18. DongIer (R).

19. Draper (H.).

20. Daune (P.).

21. Dutinov.
Das Eikonal. Abh. der math.-phys. classe der kön. sächs. Gesellschaf't der Wio senschaften. Band XXI. Leipzig, 1895.

Apparcils pour l'examen microscopique des corps opaques. Hevue gen. des Sciences, $t$. VII, 1896.

Mémoire sur les moyens de perfectionner les lunettes dapproche par l'usage d'objectifs composés de plusieurs matières diversement réfringentes, Hist. et Mém. de l'Ac. des Sc. de Paris, 17561757.

Theorie der optischen Instrumente nach Abbe. Breslau, 1894.

Traité elementrire de Physique theorique et expérimentale. édit., 1878, 4 volume. Paris, Delagrave.

Etude théorique sur les jumelles, et sur unc combinaison permettant d'en augmenter le champ et la puissance. Revue d'arlillerie, oct. 1895.

1. Vérificateurs optiques des lignes et surfaces des machines et procédés de rectification. Bull. de la Soc. d'Enconragement, juillet 1899 .

2. Sur un phacomètre a oscillations. $C$. $R$. de l'Ac. des $S_{c}$., juin 1899.

Appareil de mesure des courbures et des éléments d'un système optique quelconque. Journ. de Physique, $3^{\mathrm{e}}$ série, t. $X, 1901$.

Construclion d'un télescope à miroir argenté de 15 pouces at demi d'ouverture (1864); traduit dans les Mémoires de la Soe. d'Enc. (Supplément au Bulletin d'octobre 1905.)

Lerbuch der Optik. 1900, I.eipzig. Traduit en anglais sous le titre : Theory of optics.

Mémoire sur la détermination des indices de refraction des sept raies de Fraunhofer dans une série nombreuse de verres... Ann. de Ch. et de Phys., $3^{\text {e }}$ serie, t. XXVIII, 1850. 
22. Estiknnr (J.-E.). Note sur les Télémètres, Revue d'Artillerie, novembre 1904.

23. Euter (L.).

1. Precis de Dioptrique. Hist. et Mém. de $l$ 'Ac. des Sc. de Paris, 1765.

24. -

2. Lettres à une princesse d'Allemagne sur divers sujets de Physique el de Philosophie. Saint-Pétersbourg, 1768 1772. (Réimprimées plusieurs fois depuis.)

25. Fabat (Ch.).

Traité encyclopédique de Photographie. 8 vol., 1889-1907. Paris, Gauthier-Villars.

26. Fанит (Ch.)

et PЕ́нoт (A.). 1. Méthodes interférentielles pour la mesure des grandes épaisseurs et la comparaison des longueurs d'onde. Ann. de Ch. et de Phys., 7e série, t. XVI, 1899.

27. -

2. Sur un nouveau modèle d'interféromètre. Ann. de Ch. et de Phys., Te série, t. XXII, 1901.

28

3. Mesure en longueurs d'onde de quelques étalons de longueur's à bouts. Ann. de Ch. et de Phys., 7o série, t. XXIV, 1901.

29. FÉ (Ch.).

Méthode nouvelle pour la determination des constantes des lentilles. Hull. des seances de la Soc. fr. de Phys., 1903.

30. Forest (F.)

et Noaliat (H.). Les Bateaux sousmarins. 2 vol. Dunod, 1900.

31. Gariel (C.-M.). Études d'optique géométrique. Nony, 1889.

32. Gavss (C.-F.). Dioptrische Untersuchungen, traduites par Bhavals dans Ann. de $C h$. et de Phys., 3e série, t. XXXIII, 1851.

33. Gavt

Sur des appareils et des procédés propres à régler le tir des bouches à feu dans les balteries de côte. Rev. d'Art., t. VIII, 1876.

34. GehLER.

Physikalisches Wörterbuch., 4 vol. Leipzig, 1789. (A été réimprimé et mis a jour.)

35. Ginaud-Teulon. Sur ume nouvelle méthode et sur un nouvel instrument de télémétrie, $C$, $R$. Ac. Sc., t. I.XXX, 1875. 
36. Gligichen (A.).

1. Lerbuch der geometrischen optik. Teubner. Leipzig, 1902.

37. -

2. Leitfaden der praktischen Optik. Hirzel. Berlin, 1907.

38. Goenserts (P.-J.-L). Étude sur les prismes à réflexions intéritures. Alnales de la Soc. scient. de Bruxelles, $\mathrm{t}$. XXIV, 1900.

39. Govnen (C.-M.)- 1. Etudes théorifues et pratiques sur les levers topométriques. Memorial de l'officier du Génıe, t. XXVIII, 1892. (Publié séparément.)

40.

2. Du choix des jumelles et des soins à leur domer. Annuaire da Club alpin, 1879.

41.

3. Lunette anallatique. C. R. Ac. Sc., t. $\operatorname{LXXX}, 1875$.

42. Govi.

1. Intorno a una nuova Camera-lucida. Rendiconti delle sedute della R. Acad. dei Lincei, $4^{\mathrm{C}}$ série, t. V, 1889.

43. -

2. Della Invenzione del micrometro per gli strumenti astronomici. Bulletino di Boncompagni, t. XX, 1887.

44. -

3. Sur l'inventeur des lunettes binoculaires, G. R. Ac. Sc., t. XCI, 1880.

45. Gnubs(Sir Howano). 1. A new collimating-telescope gun-sight for large and small ordnance. The Scient. Trans. of the Roy. Dublin. Soc., $2^{\circ}$ série, t. II, $1901-1902$.

46.

2. Some new forms of gendetical instruments (môme référence que ci-dessus).

47. Hapting (H.).

1. Zur Berechuung astronomischer Fernrohrobjektive. Zeitsch. für Instrument.kunde, t. XIX, 1899.

48.

2. Ueber Astigmatismus und Bildfeldwölbung bei astronomischen Fernrohrobjektiven (même référence que cidessus).

49.

3. Zur Berechnung dreitheiliger Feinrohr-und Mikroskopobjektive. Zeit. f. Instit., t. XX, 1900.

50.

4. Zur Theorie der zweitheiligen verkittet.en Fernrohrobjektive. Zeit. f. Instk. t. XVIII, 1898 . 
51. Hatorík (E.).

52.

54.

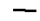

55. Hexrivaux $\langle$ J.).

56. Herschet (J.-F.-W.).

57. Hevrck (Van).

58. Hoegh (Von E.).

59. Jacob de Margr.

60. IAGHANGB.

61.

62. Lavikent (L.).
53. Helmoitz (N.).

1. Étude photométrique des images formées par les systèmes optiques. Bull. des séances de la Soc. fr. de Phys., 1905.

2. Détermination, au moyen d'un appareil photographique, du grossissement et du champ des lunettes galiléiques ou astronomiques (même référence que ci-dessus).

1. Optique physiologique. Traduction de Javal et Kuetw. Paris. Masson, 1865.

2. Die theoretische Grenze für die Leistungsfähigkeit der Mikroskope. Annalen der Physik und Chemie, 1874. Jubelband.

La Verrerie an $X X^{\circ}$ siècle, 1903.

On Light, traduit sous le titre: Traité de la Lumière, par Verhusst et Qú́TFLEF. 2 vol. 1829.

Le Microscope, 1891.

Zur Theorie der zweitheiligen verkitteten Fernrohrobjektive.Zeit.f. Instk., t. XIX, 1899.

Instruments pour la mesure des distances. Hxtrait du Mémorial de l'Artillerie de la Marine, t. VI et VII, 1878-1879. (Publié séparćment.)

1. Sur la théorie des lunetles. Nouv. Mém. de l'Acad. roy. des Sc. et BellesLeltres de Berlin, 1778, et OEuvres completes, $\mathrm{t}$. IV.

2. Mémoire sur une loi générale d'Optique. Nouv. Mem. de l'Acad. de Berlin, 1803 , et OFnvres, t. V.

Mémoire sur les méthodes et dispositifs pour le contrôle et l'exécution des surfaces optiques planes on courbes à l'usage des instruments de précision. Bull. de la Soc. d'Encourag., 1891.

63. Le Chatelizr (H.). La technique de la métallographie microscopique. Bull. de la Soc. d'Encourag., 1902.

64. Lises (A.). Histoire philosophique des progrès de la Physique. 4 vol. $1810-1813$. 
INDEX BIBLIOGRAPHIQUE

85. Kellnen (K.).

66. Martin (Ad.).

67.

69.

70. Martin (Th.).

71. Möвrus.

72. Prazmowski.

73. Porro (J.).

74. Prechte.

75. Rayleigh (lord).

76.

77.
Das orthoskopische Okular. 1849.

1. Sur une méthode d'autocollimation des objectits et son application à la mesure des indices de réfraction des verres. C. R. Ac. Sc., t. XCI, 1880 .

2. Mémoire sur les méthodes employées pour la détermination des courbures des objectifs. Ann. se. de l'Ecole norm. sup. Supplément au t. VI, $2^{\mathrm{a}}$ série, 1877.

3. Détermination des courbures d'un objectif grand angulaire pour vues. Bull. de la Soc. fr. de Photographie, 1892.

4. Méthode directe pour la détermination des courbures des objectifs de photagraphie. Bull. de la Soc. fr. de Phot,, 1893.

Sur des instruments d'optique faussement attribués aux anciens par quelques savants modernes. Bulletino di Boncompagni, t. IV, 1871.

Principales propriétés d'un système de lentilles. Nouv. Ann. de Mathémat., t. IV, 1845 (d'aprés le Journ. de Crelle, t. V, 1830).

Sur l'achromatisme chimique. C. $A$. de $l$ 'Ac. des Sc., t. LXXIX, 1874.

Note sur le micromètre parallèle indépendant. C. R. Ac. Sc., t. XLI, 1855.

Praktische Dioptrik. Wien., 1828.

1. Notes, chiefly historical, on some fundamental propositions in Optics. Philosophical magazine, $5^{\circ}$ série, $\$$ XXI, 1886.

2. On the theory of Optical images. with special reference to the microscope. Philos. Magaz., 5" série, t. XLII, 1896.

3. Investigations in Optics, with special reference to the Spectroscope, Phil. Magaz., t. VIII, 1879. 
78. Ritchey (G,-W.). Sur le télescope moderne a réflexion. Fabrication el cssais des miroirs optiques. Traduit dans les Mémoires de la Soc. d'Encourag. (Supplément au Bulletin d'octobre 1905.)

79. Roun (von).

1. Theorie und Geschichle des photographischen objektivs. Berlin, 1889.

80.

2. Die Bilderzenngung in optischen Instrumenten, vom Standpunkte der geometrischen Optik, bearbeitet von den wissenschaftlichen Mitarbeiten an der optischen Werkstätte von Carl Zeiss P. Culmann, S. Czapski, A. Könnig, F. Lüwe, M. von Rohr, H, Siengntupf, E. Wanderseeb. Berlin, J. Springer. 1904.

81. Sм1тн (R.).

Cours complet d'Optique, traduit de l'anglais de liobert Smith, avec des additions considérables, par le P. P. [P'́zzvas ]. Avignon et Paris, 1767. 2 vol.

82. Steinheil und Vorgt. Handbuch der angewandten Optik. Leipzig, Teubner. 1891.

83. Stroney.

On microscopic vision. Phil. Magaz., 5e série, t. XLII. 1896.

84. Venutr. - OEuvres.

85. Wallon.

1. Leçons d'Optique géométrique. 1900. Paris, Gauthier-Villars.

86

2. Traité élémentaire de l'objectif photographique. 1891. Paris, Gauthier-Villars.

87. WaNnersues (E.). Ueber die Verzeichnungsfehler photographischer Objektive. Zeit. f. Instk., t. XXVII. 1907.

88. Winkelmain.

Zur Demonstration der Abbeschen Theorie des Mikroskopes. Annalen der Physik., t. XIX. 1906.

\section{PRINCIPALES PUBLICATIONS PŔRIODIQUES}

Comptes rendus de l'Acaddmie des Sciences.

Journal de Physique.

Bulletin de la Société française de Physique.

Bulletin de la Société française de Photographie. 
Annales de Chimie et de Physique.

The Astrophysical Journal.

The. London, Edinburgh and Dublin Philosophical Magazine and Journal of Science.

Zeitschrift für Instrumentenkunde, avec son supplément actuel Deutsche-Mechaniler Zeitung. (Cettc publication, qui a commencé en 1880, est unique en son genre; il est regrettable qu'une revue analogue n'existe pas en France.)

On trouvera une bibliographie assez étendue dans l'ouvrage porté ci-dessus sous le $n^{0} 80$, et de nombreuses indications dans les ouvrages $n^{08} 4,25,36,53,59,67,79$.

Dans le cours de cet ouvrage, les références bibliographiques sont indiquées en caractères gras, par les nombres, entre parentheses, qui se rapportent ä l'index ci-dessus. 


\section{TABLE ALPHABETIQUE DES AUTEURS ET DES MATIËRES}

Ab8r, 55, 82, 231, 234, 239, 242. Dallemexer, 260, 264.

Aberration chromatique, 75 . Aberration sphérique, 60 .

Accommodation, 35 .

Achromatisme, 13.

Acuité visuelle, 36,56 .

AlEmbert (d') , $14,170$.

Amat, 190, 228, 2k2,326, 335 .

Anastigmat, 258.

Annalatisme, 331 .

Anneau oculaire, 91.

Aplanat, 264.

Aplanétisme, 60, 264 .

Apochromatisme, 82, 231 .

Astigmatisme, 65 .

Auto-collimation, 359 .

Axe optique, 279."

Barr et Strocd, 347, 350, 354 .

Bauernfeino, 327.

BFidAN, 339.

Binoculaire (vision), 42, 137, 241.

Biot, 14, 77, $171,317$.

Daurresse , 181, 191, 200, 275, 310.

Descahtes, 1 .

DÉY̌́, 362.

Diaphragme, 6, 110.

Diaphragme apparent, 51.

Diasporamètre, 311.

Diffraction, 50.

Iiffusiun, 61.

Dispersion rectiligne des foyers, 78.

Distorsion, 23, 126.

Dollonu, 14, 179,318 .

Doublet, 224, 262.

DUPIN, 62 .

Duplication, 333.

Eclairement, 87.

Éclat, 88, 98.

Équerre optique, 325,345 .

Estienne, 289, 303.

Euler, 13, 25, 77 .

Champ, 20, 110.

Champ apparent, 111.

Chevalieh, $224,254$.

Clamaut, 14, 168.

Clarté, 19, 8f.

Coefficient de profondeur, 151.

Coeflieient de relief, 153.

Collimateur, 285.

Coma, 67.

Convu, 236, 282.

Correction (objectif a), 238.

Courbure du champ, 118.

Fidélité des images, 22.

Flux, 86.

Focales de Sturm, 62.

Foucault, 54, 210.

Fonees, 190, 353.

Fraunhoybr, 52, 55, 168.

Frestel, 102.

Galizér, $11,300$.

Gauss, 170.

Gautier, 326, 347, 351 .

G'́RARD, 331. 
376 TABLE ALPHABÉTIQUE DES AUTEURS ET DES MATIE்RS

Granud-Tzuzor, 336.

Goknz, 200, 206, 217, 259, 285.

Goniometre, $303,311$.

Goulien, 69, 174, 282, 325, 317, 350.

Govi, $278,293$.

Grossissement, 23, 56 .

Grubs, 292.

Gunsmo, 15,81 .

Hadey, 307.

Hartivg, 171.

Héliometre, 317.

Helmholtz, 42, 43, 55.

Herschel, 40, 52, 208.

Hévélius, 198.

НоEg , 171.

Нооке, 36.

Huet, 187, 216.

HuYGRNS , $166,172$.

Image solide, $38,60,75$.

Immersion, 229, 239.

Jumelle, 137, 214.

Ketinfer, 174.

Keplen, 11, 176, 201.

Le Chatelien, 235.

Ĺ Cyнe, 355 .

Loupe, 10, $135,221$.

Lugkol, 335.

Lunette astronomique, 11, 93 , 165.

Lunettè à prismes, 183.

Lunctte-cornet, 183.

Lunette de Galilée, 11, 72, 115 , 190.

Iunette Napoléon III, 185.

Lunette panoramique, 205.

Lunette polyalde, 195.

Lunet te terrestre, 11, 176.
Mares, 62.

Mangin, 205.

Micrométre, 159, 296.

Microscope, 11, 95, 153, 225.

Millième, 303.

Mittenweg, 174.

Nachet, 231, 242, 276.

Netteté, 17, 48.

NEwToN, 13, $208,307$.

Objectif de lunette, 53, 168 , 175.

Objectif de microscope, 54, 226.

Objectif photographique, 14, 117, 244.

Oculaiı'c, 171, 180, 232.

OEil, 29.

OEil schématique, 31.

Orthoscopie, 23, 126, 249.

Ouverture numérique, $56,226$.

Périscope, 198, 205.

Pfatzyal, 255.

Photographique (plaque), 45.

Pinceau, 7, 63.

Polémoscope, 198.

Ponno, 182, 268, 314, 331.

Pouvoir séparateur, 18, 53.

Prisme de Rochon, 314.

Prisme de Wollaston, 189, 315.

Prisme en toit, 190.

Prisme newtonien, 208.

Profondeur de champ, 120.

Profondeur de foyer, 120.

Profondeur (perception de la), $43,149$.

Radiant, 52.

RaMsoen, 91, 172.

Rectiligne, rectilinéaire, 249 , 264.

Réticule, $278,280$.

Rheita, 11, 178.

Rochon, 312, 314. 
TABLE ALPHABÉTIQUB DES AUTECRS ET DES MATIÉRES 377

ScherNer, 11.

SCHOTT, 15, 81 .

Sextant, 305 .

Sovchinn, $328,335,354$.

Spectre secondaire, 81.

Stadimetre, 329.

Stanhope, 223.

Steinibil, 197, 223, 264,3 亿1.

Stéréoscopie, 43, $215,351$.

Stunu, 62.

Tache centrale, 52, 106.
Télémetre, 320.

Télébbjectif, 197, 269.

Telescope, 207.

Téléstéréoscope, 151.

Véhicule, 11, 178.

Viseur, 277.

WoLlaston, 189, 223, 221, 315.

Zkiss, 82, 171, 175, 223, 260, 285, 316. 
IRIS - LILLIAD - Université Lille 1 


\title{
TABLE SYSTÉMATIQUE DES MATIÈRES
}

\author{
INTRODUCTION
}

Généralités . . . . . . . . . . . . . . 1

Historique sommaire . . . . . . . . . . . . . . . . 8

Divisions de l'ourrage . . . . . . . . . . . . . 15

\section{PREMIÈRE PARTIE}

PROPRIÉTÉS GÉNÉRALES DES INSTRUMENTS

Chsp. I. - Des qualités optiques des instruments. . . . 17

Chap. II. - Rôle de l'cil dans la vision instrumentale . . 28

Ghar. III. - De la netteté des images dans les instruments optiques............. 48

Diffraction . . . . . . . . . . . . . 50

A berrations de sphéricité; aplanétisme . . . . . 60

Aberrations de réfrangibilité; achromatisme. . . . 75

Chap, IV, - De la clarté des instrumen/s. . . . . . . 86

Chap. V. - Du champ des instruments. . . . . . . 110

Char. VI. - De la fidélité des images; distorsion; orthoscopie. . . . . . . . . . 126

Crap. VII. - Des images dans les instruments binoculaires. . . . . . . . . . 137

Chap. VIII. - Instruments de mesure . . . . . . 156 


\section{DEUXIÈME PARTIE}

\section{ORGANISATION ET EMPLOI DES INSTRUMENTS}

Chap. IX. - Instruments d'observation à grande distance. 165 Lunette astronomique. . . . . . . . . 165 Lunette terrestre . . . . . . . . . . . 1.76 Télescopes catoptriques. . . . . . . . . . . . 207 Jumelles . . . . . . . . . . . . . . . . . 214

Chsp. X. - Instruments d'observation à faible distance. . 221

Loupe ... . . . . . . . . . . 221

Microscope. . . . . . . . . . 225

Crss. XI. - Objectif photographique. . . . . . . . . . 244

Chap. XII. - Instruments et organes de mesure. - Déterminalion des directions. . . . . . . . 277

Lunettes-viseurs . . . . . . . . . . . . . 277

Collimateurs .............. . 285

Chap. XIII. - Instruments de mesure des angles. . . . 296

Chap. XIV. - Instruments de mesure indirecte des longueurs. - Télémétres. . . . . . . 320

Lunettes stadimétriques. . . . . . . . . . . . . 329

Télémètres à décalage d’images . . . . . . . . . 333

Télémètres monostatiques. . . . . . . . . . . 336

Mesure de petits intervalles. . . . . . . . . . 357

Chap. XV. - Instruments basés sur le prineipe de l'autocollimation. . . . . . . . . 359

INDEX BIBLIOGRAPHIQUE. . . . . . . . . . . . . . . . . 367

Tabli alphabétique des aUteuns bT deg matiéres . . . . . 375

TAlih systématique des matières . . . . . . . . . 379 


\section{ENCYCLOPÉDIE SCIENTIFIQUE}

Publice sous la direction du $D$ - Toulouse

Nous avons entrepris la publication, sous la direction générale de son fondateur, le $D^{r}$ Toulouse, Directeur a l'École des Hautes-Études, d'une Excychopéde scientifique de langue française dont on mesurera l'importance à ce fait qu'elle est divisée en 40 sections ou Bibliothèques et qu'elle comprendra environ rooo volumes. Elle se propose de rivaliser avec les plus grandes encyclopédies étrangères et même de les dépasser, tout à la fois par le caractère nettement scientifique et la clarté de ses exposés, par l'ordre logique de ses divisions et par son unité, enfin par ses vastes dimensions et sa forme pratique.

PLAN GÉNÉRAL DE L'ENCYCLOPÉdIE

Mode de publication. - L'Encyclopédie se composera de monographies scientifiques, classées mélhodiquement et formant dans leur enchainement un exposé de toute la science. Organisée sur un plan systématique, cette Encyclopedie, tout en évitant les inconvénients des Traités, - massifs, d'un prix global élevé, difficiles à consulter, - et les inconvénients des Dictionnaires, où les articles scindés irrationnellement, simples chapitres alphabétiques, sont toujours nécessairement incomplets, - réunira les avantages des uns et des autres.

Du Traité, lEncyclopédie gardera la supériorité que possède 
un ensemble complet, bien divisé et fournissant sur chaque science tous les enseignements et tous les renseignements qu'on en réclame. Du Dictionnaire, l'Encyclopédie gardera les facilités de recherches par le moyen d'une table générale, l'Index de l'Encyclopédie, qui paraitra dès la publication d'un certain nombre de volumes et sera réimprimé périodiquement. L'Index renverra le lecteur aux différents volumes et aux pages où se trouvent traités les divers points d'une question.

Les éditions successives de chaque volume permettront de suivre toujours de près les progrès de la science. Et c'est par là que s'affirme la supériorité de ce mode de publication sur tout autre. Alors que, sous sa masse compacte, un traité, un dictionnaire ne peut être récedité et renouvelé que dans sa totalité et qu'à d'assez longs intervalles, inconvénients graves qu'atténuent mal des suppléments et des appendices, l'Encyclopédie scientifique, au contraire, pourra toujours rajeunir les partics qui ne seraient plus au courant des derniers travaux importants. Il est évident, par exemple, que si des livres d'algebre ou d'acoustique physique peuvent garder leur valeur pendant de nombreuses années, les ouvrages exposant les sciences en formalion, conme la chimie physique, la psychologie ou les techrologies industrielles, doivent nécessairement être remaniés à des intervalles plus courts.

Le lecteur appréciera la souplesse de publication de cette Encyclopédie, toujours vivante, qui s'élargira au fur et à mesure des besoins dans le large cadre tracé dès le début, mais qui constituera tonjours, dans son ensemble, un traité complet de la Science, dans chacune de ses sections un traité complet d'une science, et dans chacun de ses livres une monographie complète. Il pourra ainsi n'acheter que telle ou telle section de l'Encyclopédie, sûr de n'avoir pas des parties dépareillées d'un tout.

L'Encyclopédie demandera plusieurs années pour être achevée; car pour avoir des expositions bien faites, elle a pris ses collaborateurs plutôt parmi les savants que parni les professionnels de la rédaction scientifique que l'on retrouve généralement dans les cuvres similaires. Or les savants écrivent peu et lentement: ct il est préférable de laisser temporairement sans attribution certains ouvrages plutòt que de les confier à des auteurs insuffisants. Mais cette leuteur et ces vides ne présenteront pas d'in- 
convénients, puisque chaque livre est une ouvre indépendante et que tous les yolumes publićs sont à tout moment réunis par I'Index de l'Encyclopédie. On peut donc encore considérer l'Encyclopédie comme une libraipie, où les livres soigneusement choisis, au lieu de représenter le hasard d'une production individuelle, obéiraient à un plan arrèté d'avance, de manière qu'il n'y ait ni lacune dans les parties ingrates, ni duuble emploi dans les parties très cultivées.

Caractère scientifique des ouvrages. - Actuellement, les livres de science se diviscnt en deux classes bien distinctes: les livres destinés aux savants spécialisés, le plus souvent incompréhensibles pour tous les autres, faute de rappeler au début des chapitres les connaissances nécessaires, et surtout faute de définir les nombreux termes techniques incessamment forgés, ces derniers rendant un mémoire d'une science particulière inintelligible à un savant qui en a abandoné l'étude durant quelques années ; et ensuite les livres écrits paur le grand public, qui sont sans profit pour des savants et méme pour des personnes d'une certaine culture intellectuelle.

L'Encyclopédie scientifique a l'ambition de s'adresser au public le plus large. Le savant spécialisé est assuré de rencontrer dans les volumes de sa partie une mise au point très exacte de l'état actuel des questions ; car chaque Bibliothèque, par ses techniques et ses monographies, est d'abord faite avec le plus grand soin pour servir d'instrument d'études et de recherches à ceux qui cultivent la science particulière qu'elle représente, ct sa devise pourrait ĉtre : Par les savants, pour les savants. Quelques-uns de ces livres seront même, par leur caractère didactique, destinés á devenir des ouvrages classiques et à servir aux études de l'enseignement secondaire ou supérieur. Mais, d'autre part, le lecteur non spécialisé est certain de trouver, toutes les fois que ccla scra nécessaire, au seuil de la section, - dans un ou plusieurs volumes de généralités, - et au scuil du volume, - dans un chapitre particulier, - des données qui formeront une vériLable introduction le mettant à même de poursuivre avec profit sa lecture. Un vocabulaire technique, place, quand il y aura lieu, à la fiu du-volume, lui permettra de connaitre tonjours le sens des mots spéciaux. 
II

OROANISATION SCIENTIFIQUE

Par son organisation scientifique, l'Encyclopédie paraft devoir offrir aux lecteurs les meilleures garanties de compétence. Elle est divisée en Sections ou Bibliothèques, à la tête desquelles sont placés des savants professionnels spécialisés dans chaque ordre de sciences et en pleine force de production, qui, d'accord avec le Directeur général, établissent les divisions des matières, choisissent les collaborateurs et acceptent les manuscrits. Le même esprit se manifestera partout : éclectisme et respect de toutes les opinions logiques, subordination des théories aux données de l'expérience, soumission d̀ une discipline rationnelle stricte ainsi qu'aux règles d'une exposition méthodique et claire. De la sorte, le lecteur, qui aura élé intéressé par les ouvrages d'une section dont il sera l'abonné régulier, sera amené consulter avec confiance les lives des autres sections dont il aura besoin, puisqu'il sera assuré de trouver partout la même pensée et les mêmes garanties. Acluellement, en effet, il est, hors de sa spécialité, sans moyen pratique de juger de la compétence réelle des auteurs.

Pour mieux apprécier les tendances variées du travail scientifique adapté à des fins spéciales, l'Encyclopédie a sollicité, pour la direction de chaque Bibliothèque, le concours d'un savant placé dans le centre même des études du ressort. Elle a pu ainsi réunir des représentants des principaux Corps savants, Établisscments d'enseignement et de recherches de langue française :

Institut.

Académie de Médecine.

Collège de France.

Museum d'Histoire naturelle.

Ecole des Hautes-Études.

Sorbonne et École normale.

Facaltés des Sciences.

Facultés des Lettres.

Facultes de Médecine.

Instituts Pasteur.

Ecole des Ponts et Chaussies.

École des Mines.

École Polytechnique.

Gonservatoire des Arts et Métiers.

École d'Anthropologie.

Institut National agronomique.

École vétérinaire d'Alfort.

École superieure d'Électricité.

École de Chimie industrielle de Lyon.

École des Beaux-Arts.

École des Sciences politiques.

Observatoire de Paris.

Hopitaux de Paris.

IRIS - LILLIAD - Université Lille 1 


\section{BUT DE L'ENCYCLOPÉDIE}

Au xvir" siècle, "l'Encyclopédie " a marqué un magnifique mouvement de la pensée vers la critique rationnelle. A cette époque, une talle manifestation devait avoir un caractere philosophique. Aujourd'hui, l'heure est venue de renonveler ce grand effort de critique, mais dans une direction strictement scientifique; c'est là le but de la nouvelle Encyclopedie.

Ainsi la science pourra lutter avec la littérature pour la direction des esprits cultivés, qui, au sortir des écoles, ne demandent guère de conseils qu'aux cuvres d'imagination et d des encyclopédies où la science a une place restreinte, tout á fait hors de proportion avec son importance. Le moment est favorable à cette tentative; car les nouvelles générations sont plus instruites dans l'ordre scientifique que les précédentes. D'autre part, la science est devenue, par sa complexité et par les corrélations de ses parties, une matière qu'il n'est plus possible d'exposer sans la collaboration de tous les spécialistes, unis la comme le sont les producteurs dans tous les départements de l'activité économique contemporaine.

A un autre point de vue, l'Encyclopédie, embrassant toutes les manifestations scientifiques, servira comme tout inventaire \& mettre au jour les lacunes, les champs encore en friche ou abandonnés, - ce qui expliquera la lenteur avec laquelle certaines sections se développeront, - et suscitera peut-être les travaux nécessaires. Si ce résultat est atteint, elle sera fière d'y avoir contribué.

Elle apporte en outre une classification des sciences et, par ses divisions, une tentative de mesure, une limitation de chaque domaine. Dans son ensemble, elle cherchera á refléter exactement le prodigieux effort scientifique du commencement de ce siècle et un moment de sa pensée, en sorte que dans l'avenir elle reste le document principal où l'on puisse retrouver et consulter le témoignage de cetle époque intellectuelle.

On peut voir aisement que l'Encyclopėdie ainsi conçue, ainsi réalisée, aura sa place dans toutes les bibliolheques publiques, universitaires et scolaires, dans les laboratoires, entre les mains Instruments optiques. 
des savants, des industriels et de tous les hommes instruits qui veulent se tenir au courant des progrès, dans la partie qu'ils cultivent eux-mêmes ou dans tout le domaine scientifique. Elle fera jurisprudence, ce qui lui dicte le devair d'impartialité qu'elle aura à remplir.

Il n'cst plus possible de vivre dans la société moderne en ignorant les diverses formes de cette activité intellectuelle qui révolutionne les conditions de la vie; et l'interdépendance de la science ne permet plus aux savants de rester cantonnés, spécia lisés dans un étroit domaine. Il leur faut, - et cela leur est souvent difficile, - se mettre au courant des recherches voisines. A tous, l'Kncyclopédie offre un instrument unique dont la portée scientifique et sociale ne peut échapper à personne.

\section{V}

\section{CLASSIFICATION DES MATIERES SCIENTIFIQUES}

La division de l'Encyclopédie en Bibliothèques a rendu nécessaire l'adoption d'une classification des sciences, où se manifeste nécessairement un certain arbitraire, étant donné que les sciences se distinguent beaucoup moins par les différences de leurs objets que par les divergences des apercus et des habitudes de notre esprit. Il se produit en pratique des interpénétrations réciproques entre leurs domaines, en sorte que, si l'on donnait a chacun l'étendue à laquelle il peut se croire en droit de prétendre, il envahirait tous les territoires voisins; une limitation assez stricte est nécessitée par le fait même de la juxtaposition de plusieurs sciences.

Le plan choisi, sans riser à constituer une synthèse philosophique des sciences, qui ne pourrait etre que subjective, a tendu pourtant à échapper dans la mesure du possible aux habitudes traditionnelles d'esprit, particulierement a la routine didactique, et à s’inspirer de principes rationnels.

Il y a deux grandes divisions dans le plan général de l'Encyclopédie: d'un côté, les sciences pures, et, de l'autre, toutes les technologies qui correspondent à ces sciences dans la sphère des epplications. A part et au début, une Bibliothèque d'introduc- 
tion générale est consacrée à la philosophie des sciences (histoire des idées directrices, logique et méthodologie).

Les sciences pures et appliquées présentent en outre une division générale en sciences du monde inorganique et en sciences biologiques. Dans ces deux grandes catégories, l'ordre est celui de particularité croissante, qui marche parallèlement à une rigueur décroissante. Dans les sciences biologiques pures enfin, un groupe de sciences s'est trouvé mis à part, en tanl qu'elles s'occupent moins de dégager des lois générales et abstraites que de fournir des monographies d'êtres concrets, depuis la paléontologie jusqu'à l'anthropologie et l'ethnographic.

Etant donnés les principes rationnels qui ont dirigé cette classification, il n'y a pas lieu de s'étonner de voir apparaitre des groupements relativement nouveaux, une biologie générale, une physiologie et une pathologie végétales, distinctes aussi bien de la botanique que de l'agriculture, - une chimie physique, etc.

En revanche, des groupements hétérogènes se disloquent pour que leurs parties puissent prendre place dans les disciplines auxquelles elles doivent revenir. La géographie, par exemple, retourne à la géologie, et il $y$ a des géographies botanique, zoologique, anthropologique, économique, qui sont éludiées dans la botanique, la zoologie, l'anthropologie, les sciences économiques.

Les sciences médicales, immense juxtaposition de tendances très diverses, unies par une tradition utilitaire, se désagrègent en des sciences ou cles techniques précises; la pathologie, science de lois, se distingue de la thérapeutique ou de l'hygiène, qui ne sont que les applications des données générales fournies par les sciences pures, et à ce titre mises à leur place rationnelle.

Enfin, il a paru bon de renoncer à l'anthropocentrisme qui exigeait une physiologie humaine, une anatomie humaine, une embryologie humaine, une psychologie humaine. Lhomme est intégré dans la série animale dont il est un aboutissant. Et ainsi, son organisation, ses fonctions, son développement, s'éclairent de toute l'évolution antérieure et préparent I'étude des formes plus complexes des groupements organiques qui sont offerts par l'étude des sociétés. 
On peut voir que, malgré la prédominance de la préoccupation pratique dans ce classement des Bibliotheques de l'Encyclopédie scientifique, le souci de situer rationnellement les sciences dans leurs rapports réciproques n'a pas été négligé. Enfin il cst à peine besoin d'ajouter que cet ordre n'implique nullement une hiérarchie, ni dans l'importance ni dans les difficultés des diverses sciences. Certaines, qui sont placées dans la technologie, sont d'une complexité extrême, et leurs recherches peuvent figurer parmi les plus ardues.

Prix de la publication. - Les volumes, illustrés pour la plupart, seront publiés dans le format in-18 jésus et cartonnés. De dimensions commodes, ils auront 400 pages environ, ce qui représente une matière suffisante pour une monographie ajant un objet défini et important, établie du reste selon l'économie du projet qui saura éviter l'émiettement des sujets d'cxposition. Le prix étant fixé uniformément \& 5 francs, c'est un réel progrès dans les conditions de publication des ouvrages scientifiques, qui, dans certaines spécialités, coûtent encore si cher. 


\section{TABLE DES BIBLIOTHÈQUES}

Dinbcteun: Dr Toulouse, Directeur de Laboratoire a l'École des Haules śtudes. Sechítaire génénal : H. Pienon, agrége de l'Tniversite.

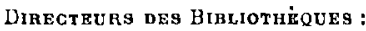

1. Philosophie dessciences. P. PaInLEv'́, de l'Institut, professeur à la Sorbonne.

I. Sctenceg runes

A. Sciences mathématiques :

2. Mathématiques. . J, Dracir, professeur a la Facultế des Sciencos de l'Université de Toulouse.

3. Mécanique. . . . J. Drach, professeur à la Faculté des Sciences de l'Université de Toulouse.

B. Sciences inorganiques:

4. Physique - . - . A. Levuc, professeur adjoint de physique à la Sorbonne.

5. Chimie physique. . . J. Pennn, chargé de cours à la Sorbonne.

6. Chimie . - . . . A. Pictet, professeur à la Faculté des Sciences de l'Université de Geneve.

7. Astronomie et Physique J. Mascart, astronome adjoint à l'Observatoiro céleste. . . . . . de Paris.

8. Météorologie. . . - B. Bnunhes, professeur a la Faculté des Sciences de l'Université de ClermontFerrand, directeur de l'Observatoire du puy de Dôme.

9. Minéralogie et Pétro- A. LAcroix, de l'lustitut, professeur au Mugraphie. . . . . séum d'Histoire naturelle.

10. Géologie. . . . . M. Bovle, professeur au Musénm d'Histoire naturelle. 
11. Océanographie physique. . . . . .

J. Richand, directeur du Musée Océanographique de Monaco.

\section{Sciences biologiques normatives :}

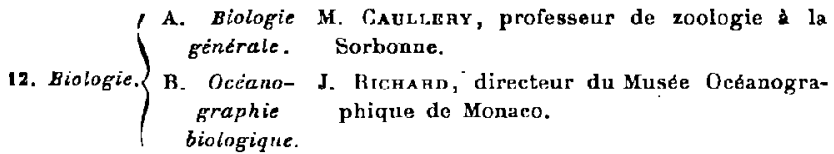

13. Physique biologique. . A. Iмвент, professeur à la Faculté de Médecine de l'Université do Montpellier.

14. Chimie biologique. G. Bertrand, professeur do chimio biologique à la Sorbonne, chef de service a l'Institut Pasteur.

15. Physiologie et Patholo- L. Mavan, de l'Institut, professeur au Muséum gie vigitales . . . d'Histoire naturelle.

16. Physiologie. . . . . J.-P. Lhnglors, professeur agrégé à la Faculté de Médecine de Paris.

17. Psychologie. . . . . E, Tourouse, directeur de Laboratoirè à l'École des Hautes Études, médecin on chef do 1'asile de Villejuif.

18. Sociologie . . . . . G. Richarn, professeur à la Faculté des Lettres de l'Université de Bordeaux.

19. Microbiologie et Parasitologie.

A. Calmetre, professeur à la Faculté de Médecine de l'Université, directeur de l'Institut Pasteur de Lille; et F. Brzançon, professeur agrégé à la Faculté de Médecine de Paris, médecin des Hôpitaux.

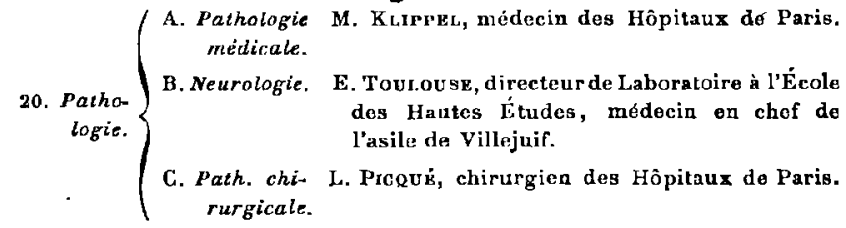

D. Sciences biologiques descriptives :

21. Paléontologie . . . M. Bause, professeur au Muséum d'Histoire uaturelle. 


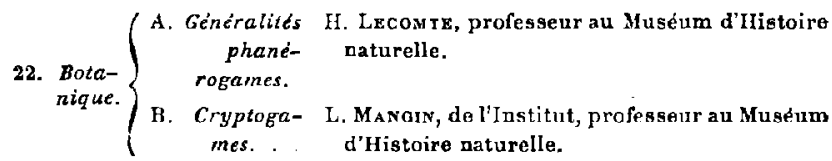

23. Zoologie. . . . . G. Lorses, directeur de Laboratoire à l'École des Hautes Études.

24. Anatomie et Embryoln- G. LoISEL, directeur de Laboratoiro à l'Écolo gie. . . . . . des Hautes Études.

25. Anthropologie et Ethno- G. PApILIAULT, directeur adjoint du Laboragraphie. . . . . toire d'Antbropologie l'ḱcole des Hautes Études, professeur à l'École d'An thropologie.

26. Économie politique. - D. HrLt.KT, secrótaire perpétuel de la sociôté d'Economie politique, professeur à l'École des Seiences politiques.

II. Sciences APpliqueks

\section{A. Sciences mathématiques :}

27. Hathematiques appli- M. D'OCAane, professeur à l'Écoledes Ponts ek qués . . . - - Chaussées, répétiteur à l'École Polytechnique.

28. Mécanique appliquée et M. D'Ochans, professeur à l'École des Ponts ot génie. . . . . Chaussées, rópétiteur àl'bicole Polytechnique.

B. Sciences inorganiques :

29. Industries physiques . H. Chatmat, sou6-directeur de l'École oupérieure d'Électricité de Paris.

30. Photographic . . - A. SzYBWETz, sous-directeur de l'École de Chimie industrielle de Lyon.

31. Indistries chimiques. . J. Dhnômв, professeur agrégé de physique au collège Chaptal, inspecteur des Établissements classés.

32. Géologie et minéralogie L. CAyxux, professeur à l'Institut national appliquées... . agronomique, professeur de géologie à l'École des Kines.

33. Construction . . . J. Pillet, professeur au Conseryatoire des Arts et Métiers et a l'École des Beaux-Arts. 
C. Sciences biologiques :

34. Industries biologiques. G. Bertrand, professeur de chimic biologiquo à la Sorbonne, chef de service à I'Institut Pasteur.

35. Botanique appliquée et H. Lecomtr, profosseur au Muséum d'Histoire agriculture. . . . naturelle.

36. Zoologie appliquée. . J. Peldegran, assistant au Muséum d'Histoire naturelle.

37. Thérapeutique génerrale

G. Poucher, membra de l'A cadémie de médeet pharmacologie. . cino, professeur a la Faculté de Médecine de l'Université de Paris.

38. Hygiène et médecine A. Casmetre, professeur à la Faculté de Mépubliques . . . . decine de l'Université, directeur de l'Institut Pasteur de Lille.

39. Psychologie appliquée. E. Tovlouse, dirceteur de Laboratoire a l'École des Hautes Études, médecin en chef de l'asile de Villejuif.

10. Saciologic appliquét. . Tн. RuYssen, professeur à la Faculté des Lettres de l'Lniversité de Bordeaux.

M. Albent Maine, bibliothécaire à Ia Sorhonne, est chargé de l'Index de l'Encyclopédie scientifique.

33830. - Tours, impr. Mame. 


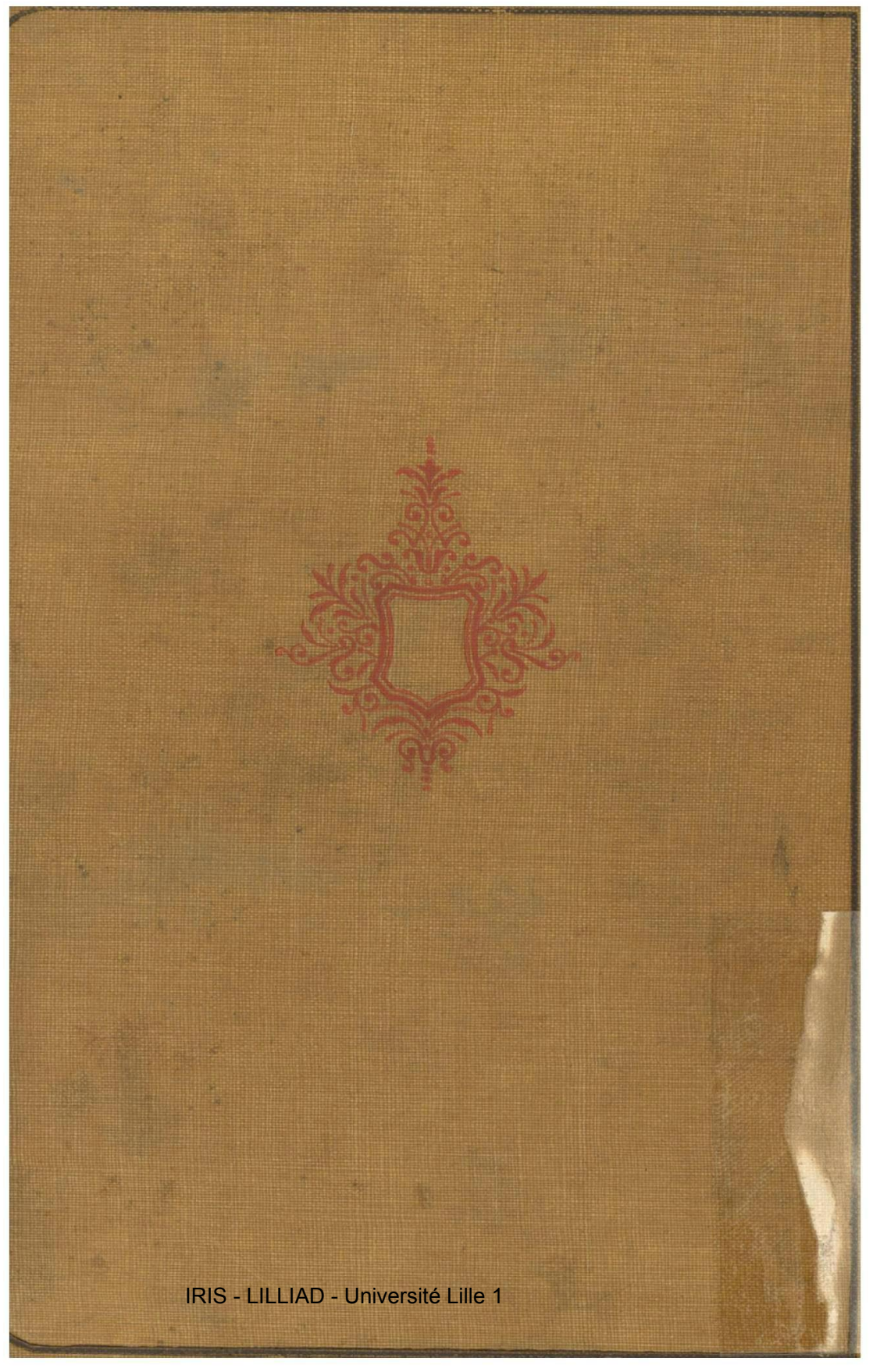

